\title{
The KPZ fixed point
}

\author{
by \\ Konstantin Matetski \\ JEREMY QUASTEL \\ University of Toronto \\ Toronto, ON, Canada \\ University of Toronto \\ Toronto, ON, Canada
}

DANIEL REMENIK

Universidad de Chile

Santiago, Chile

\section{Contents}

1. The KPZ universality class . . . . . . . . . . . . . . . . . . 116

2. TASEP . . . . . . . . . . . . . . . . 120

2.1. Biorthogonal ensembles . . . . . . . . . . . . . . . 121

2.2. Representation of the kernel as a hitting probability . . . . . 126

2.3. Formulas for TASEP with right-finite initial data . . . . . . 128

2.4. Integrability . . . . . . . . . . . . . . . . . . . 134

3. $1: 2: 3$ scaling limit . . . . . . . . . . . . . . . . . 135

3.1. State space and topology . . . . . . . . . . . . . 136

3.2. Approximation setup . . . . . . . . . . . . . . . . . . . . . . . . . . . . . . . . . . . . . . . .

3.3. Limiting operators . . . . . . . . . . . . . . . . . . 140

3.4. From 1-sided to 2-sided formulas . . . . . . . . . . . . . 146

3.5. Tightness and Markov property . . . . . . . . . . . . . . 147

4. The invariant Markov process . . . . . . . . . . . . . . . . . . . . . . . . . . . . . . . . 150

4.1. Brownian scattering theory . . . . . . . . . . . . 150

4.2. KPZ fixed point formula . . . . . . . . . . . . . . . 152

4.3. Properties of the KPZ fixed point . . . . . . . . . . . . . . 154

4.4. Recovery of the Airy processes . . . . . . . . . . . . . . 160

4.5. Variational formulas . . . . . . . . . . . . . . . . . . 162

4.6. Regularity in time . . . . . . . . . . . . . . . . 165

4.7. Equilibrium space-time covariance . . . . . . . . . . . . 166 
Appendix A. Trace norm of the fixed point kernel . . . . . . . . . . . 167

A.1. Proof of Theorem 4.1 . . . . . . . . . . . . . . . 168

A.2. Proof of Proposition $4.3 \ldots \ldots \ldots$

Appendix B. Trace-norm convergence of the rescaled TASEP kernels . 173

B.1. Estimates . . . . . . . . . . . . . . . . . . . 173

B.2. Convergence . . . . . . . . . . . . . 186

Appendix C. Finite propagation speed and regularity . . . . . . . 188

C.1. Finite propagation speed . . . . . . . . . . . . . . 188

C.2. Regularity . . . . . . . . . . . . . . . . . . . . . 189

Appendix D. Path integral formulas . . . . . . . . . . . . . . . . . 193

D.1. An alternative version of $[8$, Theorem 3.3] . . . . . . . . 193

D.2. Proof of the TASEP path integral formula . . . . . . . . . 197

Acknowledgements. . . . . . . . . . . . . . . . . . 199

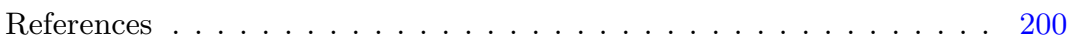

\section{The KPZ universality class}

All models in the 1-dimensional Kardar-Parisi-Zhang (KPZ) universality class (random growth models, last passage percolation and directed polymers, random stirred fluids) have an analogue of the height function $h(t, x)$ (free energy, integrated velocity) which is conjectured to converge at large time and length scales $(\varepsilon \searrow 0)$, under the KPZ 1:2:3 scaling

$$
\varepsilon^{1 / 2} h\left(\varepsilon^{-3 / 2} t, \varepsilon^{-1} x\right)-C_{\varepsilon} t
$$

to a universal fluctuating field $\mathfrak{h}(t, x)$ which does not depend on the particular model, but does depend on the initial data class. Since many of the models are Markovian, the invariant limit process, the KPZ fixed point, will be as well. The purpose of this article is to describe this Markov process, and how it arises from certain microscopic models.

The KPZ fixed point should not be confused with the KPZ equation [38],

$$
\partial_{t} h=\lambda\left(\partial_{x} h\right)^{2}+\nu \partial_{x}^{2} h+\sigma \xi
$$

with $\xi$ being a space-time white noise, which is a canonical continuum equation for random growth, lending its name to the class. One can think of the space of models in the class as having a trivial, Gaussian fixed point, the Edwards-Wilkinson fixed point, given by (1.2) with $\lambda=0$ and the 1:2:4 scaling $\varepsilon^{1 / 2} h\left(\varepsilon^{-2} t, \varepsilon^{-1} x\right)-C_{\varepsilon} t$, and the nonlinear KPZ fixed point, conjecturally given by sending $\nu \searrow 0$ in (1.2) with $\sigma=\nu^{1 / 2}$. The $\mathrm{KPZ}$ equation is just one of these many models, but it does play a distinguished role 
as the (conjecturally) unique heteroclinic orbit between the two fixed points. The KPZ equation can be obtained from certain microscopic models in the weakly asymmetric or intermediate disorder limits [1], [6], [18], [21], [22], [42] (which are not equivalent, see [30]). Since some of these models are partially solvable (in particular the asymmetric simple exclusion process, through the work of Tracy and Widom [62]-[64] exact 1-point distributions are known for the KPZ equation for special initial data [2]. These issues of the universality of the KPZ equation and its distributions comprise the weak KPZ universality conjecture.

However, the KPZ equation is not invariant under the KPZ 1:2:3 scaling (1.1), which is expected to send it, along with all other models in the class, to the true universal (strong coupling, long time) fixed point. In modelling, for example, edges of bacterial colonies, forest fires, or spread of genes, the non-linearities or noise are often not weak, and it is really the fixed point that should be used in approximations and not the KPZ equation. However, progress has been hampered by a complete lack of understanding of the time evolution of the fixed point itself. Essentially all one had was fixed-time distributions of a few special self-similar solutions, the Airy processes.

Under the KPZ 1:2:3 scaling (1.1), the coefficients of (1.2) transform as

$$
\left(\nu, \sigma^{2}\right) \longmapsto \varepsilon^{1 / 2}\left(\nu, \sigma^{2}\right) .
$$

A naive guess would then be that the fixed point is nothing but the vanishing viscosity $(\nu \searrow 0)$ solution of the Hamilton-Jacobi equation

$$
\partial_{t} h=\lambda\left(\partial_{x} h\right)^{2}+\nu \partial_{x}^{2} h
$$

given (for $\lambda>0$ ) by the Hopf-Lax formula

$$
h(t, x)=\sup _{y}\left\{-\frac{1}{4 \lambda t}(x-y)^{2}+h_{0}(y)\right\} .
$$

It is not. One of the key features of the class is a stationary solution consisting of (nontrivially) time-dependent Brownian motion height functions (or discrete versions of it). But Brownian motions are not invariant for Hopf's formula (see [27] for the computation). Our story has a stronger parallel in the dispersionless $(\nu \rightarrow 0)$ limit of the (integrated) Korteweg-de Vries (KdV) equation

$$
\partial_{t} h=\lambda\left(\partial_{x} h\right)^{2}+\nu \partial_{x}^{3} h
$$

Brownian motions are invariant for all $\nu$ (at least in the periodic case [52]). But, as far as we are aware, the zero-dispersion limit has only been done on a case-by-case basis, 
with no general formulas. One can imagine that the various schemes lead to different weak solutions of the ill-posed Hamilton-Jacobi equation

$$
\partial_{t} h=\lambda\left(\partial_{x} h\right)^{2},
$$

with only the vanishing viscosity solution being characterized so far, through the entropy condition in its various manifestations. However, in our situation, where $h(t, x)$ is locally Brownian in $x$, it is far from clear that the notion of weak solution can have any meaning whatsoever.

The KPZ fixed point is given by a variational formula (see Theorem 4.18), analogous to (1.3), but with a residual forcing noise, the Airy sheet. Unfortunately, our techniques do not allow us to characterize this noise. Instead, we obtain a complete description of the Markov field $\mathfrak{h}(t, x)$ itself through the exact calculation of its transition probabilities. These transition probabilities are given in (4.7) and define the invariant Markov process.

The strong KPZ universality conjecture (still wide open) is that this fixed point is the limit under the scaling (1.1) for any model in the class, loosely characterized by having (1) local dynamics, (2) smoothing mechanism, (3) slope dependent growth rate (lateral growth), (4) space-time random forcing with rapid decay of correlations. Alternatively, convergence to the fixed point can be taken as the definition of the KPZ universality class.

Universal fixed points are a theme in probability and statistical physics: 2-dimensional critical Ising model, Schramm-Loewner evolution, Liouville quantum gravity/Brownian map, the Brownian web, and the continuum random tree have offered asymptotic descriptions for huge classes of models. In general, these have been obtained as non-linear transformations of Brownian motions or Gaussian free fields, and their description relies to a large degree on symmetry - often conformal invariance.

In the case of $\phi_{d}^{4}[58]$, the main tool is perturbation theory. Even the recent theory of regularity structures [29], which makes sense of the KPZ equation (1.2), does so by treating the non-linear term as a kind of perturbation of the linear equation.

In our case, we have a non-perturbative 2-dimensional field theory with a skew symmetry, and a solution should not in principle even be expected. What saves us is the 1-dimensionality of the fixed-time problem, and the fact that several discrete models in the class have an explicit description using non-intersecting paths. Here, we work with TASEP, obtaining a complete description of the transition probabilities in a form which allows us to pass transparently to the 1:2:3 scaling limit. $\left({ }^{1}\right)$ In a sense, a recipe for the solution of TASEP has existed since the work of [54], who discovered a

$\left({ }^{1}\right)$ The method works for several variants of TASEP which also have a representation through biorthogonal ensembles, such as discrete time TASEPs and PushASEP, see [41]. 
highly non-obvious representation in terms of non-intersecting paths which can in turn be studied using biorthogonal ensembles [10]. However, the biorthogonalization was only implicit, and one had to rely on exact solutions for a couple of special initial conditions to obtain the asymptotic Tracy-Widom distributions $F_{\mathrm{GUE}}$ and $F_{\mathrm{GOE}}$ [60], [61], the BaikRains distribution $F_{\mathrm{BR}}$ [5], and their spatial versions, the Airy processes [3], [9], [10], [33], [34], [54]. In this article, motivated by the probabilistic interpretation of the path integral forms of the kernels in the Fredholm determinant formulas for these processes, and exploiting the skew time reversibility of TASEP, we are able to obtain a general formula in which the TASEP kernel is given by a transition probability of a random walk forced to hit the initial data.

We end this introduction with an outline of the paper and a brief summary of our results. $\S 2.1$ recalls and solves the biorthogonal representation of TASEP, motivated by the path integral representation, which is derived in the form we need it in Appendix D.2. The biorthogonal functions appearing in the resulting Fredholm determinants are then recognized as hitting probabilities in $\S 2.2$, which allows us to express the kernels in terms of expectations of functionals involving a random walk forced to hit the initial data. The determinantal formulas for TASEP with arbitrary right-finite initial conditions are in Theorem 2.6. In $\S 3$, we pass to the KPZ 1:2:3 scaling limit to obtain determinantal formulas for transition probabilities of the KPZ fixed point, which is defined formally in Definition 3.12. This limit is computed using right-finite initial TASEP data, but since we have exact formulas, we can obtain a very strong estimate (Lemma 3.4) on the propagation speed of information which allows us to show that there is no loss of generality in doing so. We then work in $\S 3.5$ to show that the Chapman-Kolmogorov equations hold. This is done by obtaining a uniform bound on the local Hölder $\beta<\frac{1}{2}$ norm of the approximating Markov fields. The proof is in Appendix C.2. §4 opens with the introduction of the Brownian scattering transform, which is the main ingredient in our Fredholm determinant formulas for the KPZ fixed point, while $\S 4.2$ gives the general formulas for the transition probabilities of the KPZ fixed point (in (4.7) and Proposition 4.3); readers mostly interested in the physical implications may wish to skip directly there. The rest of $\S 4$ gives the key properties of the KPZ fixed point: regularity in space and time and local Brownian behavior, various symmetries, variational formulas in terms of the Airy sheet, and equilibrium space-time covariance; we also show how to recover some of the classical Airy processes from our formulas. $\S 3$ and $\S 4$ are done at the level of pointwise convergence of kernels. The convergence of the kernels is upgraded to trace class in Appendix B, where the remaining technical details are filled in.

So, in a sense, everything follows once one is able to explictly biorthogonalize TASEP. We begin there. 


\section{TASEP}

The totally asymmetric simple exclusion process (TASEP) consists of particles with positions

$$
\ldots<X_{t}(2)<X_{t}(1)<X_{t}(0)<X_{t}(-1)<X_{t}(-2)<\ldots \quad \text { on } \mathbb{Z} \cup\{-\infty, \infty\}
$$

performing totally asymmetric nearest neighbour random walks with exclusion: Each particle independently attempts jumps to the neighbouring site to the right at rate 1 , the jump being allowed only if that site is unoccupied (see [40] for the non-trivial fact that the process with an infinite number of particles makes sense). Placing a (necessarily infinite) number of particles at $\pm \infty$ allows for left- or right-finite data with no change of notation, the particles at $\pm \infty$ playing no role in the dynamics. We follow the standard practice of ordering particles from the right; for right-finite data the rightmost particle is labelled 1 , unless indicated otherwise. Let

$$
X_{t}^{-1}(u)=\min \left\{k \in \mathbb{Z}: X_{t}(k) \leqslant u\right\}
$$

denote the label of the rightmost particle which sits to the left of, or at, $u$ at time $t$. The TASEP height function associated with $X_{t}$ is given, for $z \in \mathbb{Z}$, by

$$
h_{t}(z)=-2\left(X_{t}^{-1}(z-1)-X_{0}^{-1}(-1)\right)-z,
$$

which fixes $h_{0}(0)=0$.

The height function is a simple random walk path

$$
h_{t}(z+1)=h_{t}(z)+\hat{\eta}_{t}(z)
$$

with

$$
\hat{\eta}_{t}(z)= \begin{cases}1, & \text { if there is a particle at } z \text { at time } t, \\ -1, & \text { if there is no particle at } z \text { at time } t .\end{cases}
$$

The dynamics of $h_{t}$ is that local maximums become local minimums at rate 1 ; i.e., if $h_{t}(z)=h_{t}(z \pm 1)+1$ then $h_{t}(z) \mapsto h_{t}(z)-2$ at rate 1 , the rest of the height function remaining unchanged. One can think of independent rate-1 Poisson processes, one for each site $z \in \mathbb{Z}$. At the jump time of the Poisson process at $z$, we check to see if the height function has a local maximum there. If it is, we flip it to a local minimum. We can also easily extend the height function to a continuous function of $x \in \mathbb{R}$ by linearly interpolating between the integer points. The evolution of an initial height function $h$ is a deterministic function of the underlying Poisson processes, providing a coupling of the evolution from different initial conditions, which preserves the partial order $h \preceq \tilde{h}$ if $h(x) \leqslant \tilde{h}(x)$ for all $x$. 


\subsection{Biorthogonal ensembles}

TASEP was first solved by Schütz [56] using the coordinate Bethe ansatz. He showed that the transition probability for $N$ particles has a determinantal form,

$$
\mathbb{P}\left(X_{t}(1)=x_{1}, \ldots, X_{t}(N)=x_{N}\right)=\operatorname{det}\left(F_{i-j}\left(t, x_{N+1-i}-X_{0}(N+1-j)\right)\right)_{1 \leqslant i, j \leqslant N},
$$

with

$$
F_{n}(t, x)=\frac{(-1)^{n}}{2 \pi i} \oint_{\Gamma_{0,1}} d w \frac{(1-w)^{-n}}{w^{x-n+1}} e^{t(w-1)},
$$

where $\Gamma_{0,1}$ is any positively oriented simple loop which includes $w=0$ and $w=1$. To mesh with our convention of infinitely many particles, we can place particles $X_{0}(j)$, $j \leqslant 0$ at $\infty$ and $X_{0}(j), j>N$ at $-\infty$. Remarkable as it is, this formula is not conducive to asymptotic analysis, where we want to consider the later positions of $M \ll N$ of the particles; one has to find an effective way to sum over the positions of the other $N-M$ particles and, at the same time, to get rid of the dependence in $N$ (which needs to go to infinity) of the dimension of the determinant. This was overcome by [10], [54], who were able to rewrite the right-hand side of (2.2) in terms of a certain Lindström-GesselViennot/Karlin-McGregor scheme [28], [39], involving a (signed) non-intersecting line ensemble, and from that obtain the desired probabilities implicitly from the following biorthogonalization problem.

First, for a fixed vector $a \in \mathbb{R}^{m}$ and indices $n_{1}<\ldots<n_{m}$, we introduce the functions

$$
\chi_{a}\left(n_{j}, x\right)=\mathbf{1}_{x>a_{j}} \quad \text { and } \quad \bar{\chi}_{a}\left(n_{j}, x\right)=\mathbf{1}_{x \leqslant a_{j}},
$$

which we also regard as multiplication operators acting on the space $\ell^{2}\left(\left\{n_{1}, \ldots, n_{m}\right\} \times \mathbb{Z}\right)$ (and later on $\left.L^{2}\left(\left\{\mathbf{x}_{1}, \ldots, \mathbf{x}_{m}\right\} \times \mathbb{R}\right)\right)$. We will use the same notation if $a$ is a scalar, writing

$$
\chi_{a}(x)=1-\bar{\chi}_{a}(x)=\mathbf{1}_{x>a} .
$$

THEOREM 2.1. ([10]) Suppose that TASEP starts with particles labeled $1,2, \ldots$ (so that, in particular, there is a rightmost particle $)\left({ }^{2}\right),\left({ }^{3}\right)$ and let $n_{1}, \ldots, n_{m}$ be distinct positive integers. Then, for $t>0$, we have

$$
\mathbb{P}\left(X_{t}\left(n_{j}\right)>a_{j}, j=1, \ldots, m\right)=\operatorname{det}\left(I-\bar{\chi}_{a} K_{t} \bar{\chi}_{a}\right)_{\ell^{2}\left(\left\{n_{1}, \ldots, n_{m}\right\} \times \mathbb{Z}\right)},
$$

$\left({ }^{2}\right)$ We are assuming here that $X_{0}(1)<\infty$ (and thus $X_{0}(j)<\infty$ for all $j>1$ too); particles at $-\infty$ are allowed.

$\left({ }^{3}\right)$ The result in [10] is stated only for initial conditions with finitely many particles, but the extension to right-finite (infinite) initial conditions is straightforward because, given fixed indices $n_{1}<$ $n_{2}<\ldots<n_{m}$, the distribution of $\left(X_{t}\left(n_{1}\right), \ldots, X_{t}\left(n_{m}\right)\right)$ does not depend on the initial positions of the particles with indices beyond $n_{m}$. 
where det is the Fredholm determinant (see (A.1) for the definition),

$$
K_{t}\left(n_{i}, x_{i} ; n_{j}, x_{j}\right)=-Q^{n_{j}-n_{i}}\left(x_{i}, x_{j}\right) \mathbf{1}_{n_{i}<n_{j}}+\sum_{k=1}^{n_{j}} \Psi_{n_{i}-k}^{n_{i}}\left(x_{i}\right) \Phi_{n_{j}-k}^{n_{j}}\left(x_{j}\right),
$$

and where $\left(^{4}\right)$

$$
Q(x, y)=\frac{1}{2^{x-y}} \mathbf{1}_{x>y}
$$

and, $\left({ }^{5}\right)$ for $k \leqslant n-1$,

$$
\Psi_{k}^{n}(x)=\frac{1}{2 \pi i} \oint_{\Gamma_{0}} d w \frac{(1-w)^{k}}{2^{x-X_{0}(n-k)} w^{x+k+1-X_{0}(n-k)}} e^{t(w-1)},
$$

where $\Gamma_{0}$ is any positively oriented simple loop including the pole at $w=0$, but not the one at $w=1$. The functions $\Phi_{k}^{n}(x), k=0, \ldots, n-1$, are defined implicitly by

(1) the biorthogonality relation

$$
\sum_{x \in \mathbb{Z}} \Psi_{k}^{n}(x) \Phi_{\ell}^{n}(x)=\mathbf{1}_{k=\ell} ;
$$

(2) the fact that $2^{-x} \Phi_{k}^{n}(x)$ is a polynomial of degree at most $n-1$ in $x$ for each $k$.

The initial data appear in a simple way in the $\Psi_{k}^{n}$, which can be computed explicitly. The powers of $Q$ are easily computed:

$$
Q^{m}(x, y)=\frac{1}{2^{x-y}}\left(\begin{array}{c}
x-y-1 \\
m-1
\end{array}\right) \mathbf{1}_{x \geqslant y+m} .
$$

Moreover, as operators on $\ell^{2}(\mathbb{Z}), Q$ and $Q^{m}$ are invertible:

$$
Q^{-1}(x, y)=2 \cdot \mathbf{1}_{x=y-1}-\mathbf{1}_{x=y} \quad \text { and } \quad Q^{-m}(x, y)=(-1)^{y-x+m} 2^{y-x}\left(\begin{array}{c}
m \\
y-x
\end{array}\right) .
$$

It is not hard to check [10, equation 3.22] that, for all $m, n \in \mathbb{Z}$,

$$
Q^{n-m} \Psi_{n-k}^{n}=\Psi_{m-k}^{m},
$$

so, in particular, $\Psi_{k}^{n}=Q^{-k} \Psi_{0}^{n-k}$. We introduce

$$
R_{t}=e^{-(t / 2)\left(I+\nabla^{-}\right)}, \quad \text { with } \nabla^{-} f(x)=f(x)-f(x-1),
$$

$\left({ }^{4}\right)$ We have conjugated the kernel $K_{t}$ from [10] by $2^{x}$ for later convenience (see (2.12) and the discussion following it). The additional $X_{0}(n-k)$ in the power of 2 in the $\Psi_{k}^{n}$ 's has also been added for convenience, and is allowed because it just means that the $\Phi_{k}^{n}$ 's have to be multiplied by $2^{X_{0}(n-k)}$.

$\left({ }^{5}\right)$ Note that, from $(2.3)$ and $(2.7), \Psi_{k}^{n}(x)=(-1){ }^{k} 2^{X_{0}(n-k)-x} F_{-k}\left(t, x-X_{0}(n-k)\right)$ for $k \geqslant 0$. 
which can also be defined through its integral kernel (valid for all $t \in \mathbb{R}$ )

$$
R_{t}(x, y)=e^{-t} \frac{t^{x-y}}{2^{x-y}(x-y) !} \mathbf{1}_{x \geqslant y}=\frac{1}{2 \pi i} \oint_{\Gamma_{0}} d w \frac{e^{t(w-1)}}{2^{x-y} w^{x-y+1}} .
$$

Observe that $\Psi_{0}^{n}=R_{t} \delta_{X_{0}(n)}$, with $\delta_{y}(x)=\mathbf{1}_{x=y}$. We have that $Q$ and $R_{t}$ commute, because the kernels $Q(x, y)$ and $R_{t}(x, y)$ only depend on $x-y$, and thus we obtain the decomposition

$$
\Psi_{k}^{n}=R_{t} Q^{-k} \delta_{X_{0}(n-k)} .
$$

The $\Phi_{k}^{n}$, on the other hand, are defined only implicitly through (1) and (2). Only for a few special cases of initial data (step, see e.g. [24]; and periodic [9]-[11]) were they known, and hence only for those choices asymptotics could be performed in the TASEP and related cases, leading to the Tracy-Widom $F_{\mathrm{GUE}}$ and $F_{\mathrm{GOE}}$ 1-point distributions, and then later to the Airy processes for multipoint distributions.

We are now going to solve for the $\Phi_{k}^{n}$ for any initial data. Let us explain how this can be done starting just from the solution for step initial data $\left(X_{0}(i)=-i, i \geqslant 1\right)$. The derivation is based on two main ingredients. The first is a path integral version of the extended kernel formula (2.5) for the TASEP finite-dimensional distributions (see Appendix D.2 for the proof):

$$
\begin{aligned}
& \mathbb{P}\left(X_{t}\left(n_{j}\right)>a_{j} \text { for } j=1, \ldots, m\right) \\
& \quad=\operatorname{det}\left(I-K_{t}^{\left(n_{m}\right)}\left(I-Q^{n_{1}-n_{m}} \chi_{a_{1}} Q^{n_{2}-n_{1}} \chi_{a_{2}} \ldots Q^{n_{m}-n_{m-1}} \chi_{a_{m}}\right)\right)_{L^{2}(\mathbb{Z})}
\end{aligned}
$$

for $n_{1}<n_{2}<\ldots<n_{m}$, where $K_{t}^{(n)}=K_{t}(n, \cdot ; n, \cdot)$. Such formulas were first obtained in [45] for the Airy 2 process (see also [47, Appendix A]), and later extended to the Airy 1 process in [48], and then to a very wide class of processes in [8]. The key is to recognize the kernel $Q(x, y)$ as the transition probabilities of a random walk (which is why we conjugated the [10] kernel by $2^{x}$, and then $\chi_{a_{1}} Q^{n_{2}-n_{1}} \chi_{a_{2}} \ldots Q^{n_{m}-n_{m-1}} \chi_{a_{m}}(x, y)$ as the probability that this walk goes from $x$ to $y$ in $n_{m}-n_{1}$ steps, staying above $a_{1}$ at time $n_{1}$, above $a_{2}$ at time $n_{2}$, etc.

The second ingredient is the skew time reversibility of TASEP. From the description just after (2.1), it is clear that the evolution rule for the height function backwards in time is the same as that of minus the height function forward in time. We use it in the form $\left({ }^{6}\right)$

$$
\mathbb{P}_{f}\left(h_{t}(x) \leqslant g(x) \text { for } x \in \mathbb{Z}\right)=\mathbb{P}_{-g}\left(h_{t}(x) \leqslant-f(x) \text { for } x \in \mathbb{Z}\right),
$$

$\left({ }^{6}\right)$ Evolve an initial height function $f$ forward through a realization of the Poisson processes from time 0 to $t$, and call the result $h_{0 \rightarrow t}(f)$ (see the paragraph right after (2.1)). Evolve $g$ backwards through the same realization from time $t$ to 0 to obtain $h_{t \rightarrow 0}(g)$. Both maps preserve the partial order $h \preceq \tilde{h}$, so we have, for each fixed realization of the Poisson processes, $h_{0 \rightarrow t}(f) \preceq g$ if and only if $f \preceq h_{t \rightarrow 0}(g)$. On the other hand, the standard time reversibility property for TASEP says that $h_{t \rightarrow 0}(g)$ has the same distribution as $-h_{0 \rightarrow t}(-g)$. This proves $(2.13)$. 
the subscript indicating the initial data.

Suppose that we have the solution (2.6) for step initial data centered at $x_{0}$, which means that $h_{0}(x)$ is the peak $-\left|x-x_{0}\right|$. The multipoint distribution at time $t$ is given by (2.12), but we can use (2.13) to reinterpret it as the 1-point distribution of $h_{t}$ at $x_{0}$, starting from an initial condition built out of a series of $m$ peaks centered at $n_{1}, \ldots, n_{m}$ with heights $-a_{1}, \ldots,-a_{m}$. From this, we can guess a formula for the multipoint distributions by extending the resulting kernel in the usual way, as in (2.6). This last step is not fully justified at this stage, but we can use the resulting formula to simply guess the form of the biorthogonal functions $\Phi_{k}^{n}$, based on the representation of the kernel in (2.12) in terms of the hitting probability for a random walk. Theorem 2.1 is then set up perfectly, because it allows us to easily prove that the guess is correct.

This gives us our key result.

THEOREM 2.2. Fix $0 \leqslant k<n$ and consider particles at $X_{0}(1)>X_{0}(2)>\ldots>X_{0}(n)$. Let $h_{k}^{n}(\ell, z)$ be the unique solution to the initial-boundary-value problem for the backwards heat equation

$$
\begin{cases}\left(Q^{*}\right)^{-1} h_{k}^{n}(\ell, z)=h_{k}^{n}(\ell+1, z), & \ell<k, z \in \mathbb{Z}, \\ h_{k}^{n}(k, z)=2^{z-X_{0}(n-k)}, & z \in \mathbb{Z}, \\ h_{k}^{n}\left(\ell, X_{0}(n-\ell)\right)=0, & \ell<k .\end{cases}
$$

Then, the functions $\Phi_{k}^{n}$ from Theorem 2.1 are given by

$$
\Phi_{k}^{n}(z)=\left(R_{t}^{*}\right)^{-1} h_{k}^{n}(0, \cdot)(z)=\sum_{y \in \mathbb{Z}} h_{k}^{n}(0, y) R_{t}^{-1}(y, z) .
$$

Here, $Q^{*}(x, y)=Q(y, x)$ is the kernel of the adjoint of $Q$ (and likewise for $\left.R_{t}^{*}\right)$.

Remark 2.3. It is not true in general that

$$
Q^{*} h_{k}^{n}(\ell+1, z)=h_{k}^{n}(\ell, z) .
$$

In fact, $Q^{*} h_{k}^{n}(k, z)$ is divergent.

Proof. The existence and uniqueness of solutions of (2.14a)-(2.14c) is an elementary consequence of the fact that $\operatorname{ker}\left(Q^{*}\right)^{-1}$ has dimension 1 and it is spanned by the function $2^{z}$, which allows us to march forwards from the initial condition $h_{k}^{n}(k, z)=2^{z-X_{0}(n-k)}$ uniquely solving the boundary value problem $h_{k}^{n}\left(\ell, X_{0}(n-\ell)\right)=0$ at each step. $\left({ }^{7}\right)$

$\left({ }^{7}\right)$ As a linear operator, $Q^{*}$ acts on $\ell^{1}(\mathbb{Z})$, and it is there that $Q^{*}$ is invertible, with inverse $\left(Q^{*}\right)^{-1}$ defined by (2.8). However, (2.14a)-(2.14c) are being solved in the space of all sequences, in which the matrix $\left(Q^{*}\right)^{-1}$ does have a non-trivial kernel. 
Before turning to the proof of $(2.15)$ we need to prove that $2^{-x} h_{k}^{n}(0, x)$ is a polynomial of degree at most $k$. We proceed by induction. Note first that, by (2.14b), $2^{-x} h_{k}^{n}(k, x)$ is a polynomial of degree zero. Assume now that $\tilde{h}_{k}^{n}(\ell, x):=2^{-x} h_{k}^{n}(\ell, x)$ is a polynomial of degree at most $k-\ell$ for some $0<\ell \leqslant k$. By (2.14a) and (2.8), we have

$$
\tilde{h}_{k}^{n}(\ell, y)=2^{-y}\left(Q^{*}\right)^{-1} h_{k}^{n}(\ell-1, y)=\tilde{h}_{k}^{n}(\ell-1, y-1)-\tilde{h}_{k}^{n}(\ell-1, y) .
$$

Taking $x \geqslant X_{0}(n-\ell+1)$ and summing (2.16) gives

$$
\tilde{h}_{k}^{n}(\ell-1, x)=-\sum_{y=X_{0}(n-\ell+1)+1}^{x} \tilde{h}_{k}^{n}(\ell, y),
$$

due to $(2.14 \mathrm{c})$, which by the inductive hypothesis is a polynomial of degree at most $k-\ell+1$ in $x$. Similarly, taking $x<X_{0}(n-\ell+1)$, we get

$$
\tilde{h}_{k}^{n}(\ell-1, x)=\sum_{y=x+1}^{X_{0}(n-\ell+1)} \tilde{h}_{k}^{n}(\ell, y),
$$

which again is a polynomial of degree at most $k-\ell+1$. The two polynomials are the same, and thus the claim follows.

We now check the biorthogonality condition (1). Using (2.11), we get

$$
\begin{aligned}
\sum_{z \in \mathbb{Z}} \Psi_{\ell}^{n}(z) \Phi_{k}^{n}(z) & =\sum_{z_{1}, z_{2} \in \mathbb{Z}} \sum_{z \in \mathbb{Z}} R_{t}\left(z, z_{1}\right) Q^{-\ell}\left(z_{1}, X_{0}(n-\ell)\right) h_{k}^{n}\left(0, z_{2}\right) R_{t}^{-1}\left(z_{2}, z\right) \\
& =\sum_{z \in \mathbb{Z}} Q^{-\ell}\left(z, X_{0}(n-\ell)\right) h_{k}^{n}(0, z) \\
& =\left(Q^{*}\right)^{-\ell} h_{k}^{n}\left(0, X_{0}(n-\ell)\right),
\end{aligned}
$$

where, in the first equality, we have used the decay of $R_{t}$ and the fact that $2^{-x} h_{k}^{n}(0, x)$ is a polynomial together with the fact that the $z_{1}$ sum is finite to apply Fubini. For $\ell \leqslant k$, we use the boundary condition $h_{k}^{n}\left(\ell, X_{0}(n-\ell)\right)=\mathbf{1}_{\ell=k}$, which is both $(2.14 \mathrm{~b})$ and $(2.14 \mathrm{c})$, to get

$$
\left(Q^{*}\right)^{-\ell} h_{k}^{n}\left(0, X_{0}(n-\ell)\right)=h_{k}^{n}\left(\ell, X_{0}(n-\ell)\right)=\mathbf{1}_{k=\ell} .
$$

For $\ell>k$, we use $(2.14 \mathrm{a}),(2.14 \mathrm{~b})$, and $2^{z} \in \operatorname{ker}\left(Q^{*}\right)^{-1}$ :

$$
\left(Q^{*}\right)^{-\ell} h_{k}^{n}\left(0, X_{0}(n-\ell)\right)=\left(Q^{*}\right)^{-(\ell-k-1)}\left(Q^{*}\right)^{-1} h_{k}^{n}\left(k, X_{0}(n-\ell)\right)=0 .
$$

To finish the proof, we need to show that $\Phi_{k}^{n}$ satisfies condition (2) of Theorem 2.1. By (2.10), we have

$$
2^{-x} \Phi_{k}^{n}(x)=\sum_{y \geqslant 0} \frac{e^{t}}{y !}(-t)^{y} 2^{-(x+y)} h_{k}^{n}(0, x+y) .
$$

It is enough to note then that, since $2^{-z} h_{k}^{n}(0, z)$ is a polynomial of degree at most $k$, this sum is absolutely convergent and is a polynomial of degree at most $k$ in $x$ as well. 


\subsection{Representation of the kernel as a hitting probability}

Let

$$
G_{0, n}\left(z_{1}, z_{2}\right)=\sum_{k=0}^{n-1} Q^{n-k}\left(z_{1}, X_{0}(n-k)\right) h_{k}^{n}\left(0, z_{2}\right)
$$

, where $h_{k}^{n}$ is the solution of (2.14). Then, from Theorems 2.1 and 2.2, and using (2.11), we have

$$
K_{t}\left(n_{i}, \cdot ; n_{j}, \cdot\right)=-Q^{n_{j}-n_{i}} \mathbf{1}_{n_{i}<n_{j}}+R_{t} Q^{-n_{i}} G_{0, n_{j}} R_{t}^{-1} .
$$

Below the "curve" $\left(X_{0}(n-\ell)\right)_{\ell=0}^{n-1}$, the functions $h_{k}^{n}(\ell, z)$ have an important physical interpretation. $Q^{*}(x, y)$ are the transition probabilities of a random walk $B_{m}^{*}$ with Geom $\left[\frac{1}{2}\right]$ jumps (strictly) to the right. $\left(^{8}\right)$ For $0 \leqslant \ell \leqslant k \leqslant n-1$, define stopping times

$$
\tau^{\ell, n}=\min \left\{m \in\{\ell, \ldots, n-1\}: B_{m}^{*}>X_{0}(n-m)\right\}
$$

with the convention that $\min \varnothing=\infty$. Then, for $z \leqslant X_{0}(n-\ell)$, we have

$$
h_{k}^{n}(\ell, z)=\mathbb{P}_{B_{\ell-1}^{*}=z}\left(\tau^{\ell, n}=k\right) .
$$

This can be proved by checking that, with this definition, $h_{k}^{n}(\ell, z)$ satisfies $(2.14 \mathrm{~b})$ and $(2.14 \mathrm{c})$ while, for $z \leqslant X_{0}(n-\ell-1)$, it also satisfies $(2.14 \mathrm{a})$ and it is given by $2^{z}$ times a polynomial in $z$ of degree at most $n-1$; the conclusion now follows from the fact, shown in the proof of Theorem 2.2, that $2^{-z} h_{k}^{n}(\ell, z)$ is a polynomial of degree at most $n-1$.

From the memoryless property of the geometric distribution, we have for all $z \leqslant X_{0}(n)$ that

$$
\mathbb{P}_{B_{-1}^{*}=z}\left(\tau^{0, n}=k, B_{k}^{*}=y\right)=2^{X_{0}(n-k)-y} \mathbb{P}_{B_{-1}^{*}=z}\left(\tau^{0, n}=k\right)
$$

and as a consequence we get, for $z_{2} \leqslant X_{0}(n)$,

$$
\begin{aligned}
G_{0, n}\left(z_{1}, z_{2}\right) & =\sum_{k=0}^{n-1} \mathbb{P}_{B_{-1}^{*}=z_{2}}\left(\tau^{0, n}=k\right)\left(Q^{*}\right)^{n-k}\left(X_{0}(n-k), z_{1}\right) \\
& =\sum_{k=0}^{n-1} \sum_{z>X_{0}(n-k)} \mathbb{P}_{B_{-1}^{*}=z_{2}}\left(\tau^{0, n}=k, B_{k}^{*}=z\right)\left(Q^{*}\right)^{n-k-1}\left(z, z_{1}\right) \\
& =\mathbb{P}_{B_{-1}^{*}=z_{2}}\left(\tau^{0, n}<n, B_{n-1}^{*}=z_{1}\right),
\end{aligned}
$$

which is the probability for the walk starting at $z_{2}$ at time -1 to end up at $z_{1}$ after $n$ steps, having hit the curve $\left(X_{0}(n-m)\right)_{m=0, \ldots, n-1}$ in between.

$\left.{ }^{8}\right)$ We use the notation $B_{m}^{*}$ to distinguish it from the walk $B_{m}$ with transition matrix $Q$ which will appear shortly. 
The next step is to obtain an expression along the lines of (2.20) which holds for all $z_{2}$, and not just $z_{2} \leqslant X_{0}(n)$. We begin by observing that, for each fixed $y_{1}$ and $n \geqslant 1$, $2^{-y_{2}} Q^{n}\left(y_{1}, y_{2}\right)$ extends in $y_{2}$ to a polynomial $2^{-y_{2}} \bar{Q}^{(n)}\left(y_{1}, y_{2}\right)$ of degree $n-1$ with

$$
\bar{Q}^{(n)}\left(y_{1}, y_{2}\right)=\frac{1}{2 \pi i} \oint_{\Gamma_{0}} d v \frac{(1+v)^{y_{1}-y_{2}-1}}{2^{y_{1}-y_{2}} v^{n}}=\frac{\left(y_{1}-y_{2}-1\right)_{n-1}}{2^{y_{1}-y_{2}}(n-1) !},
$$

where $(x)_{k}=x(x-1) \ldots(x-k+1)$ for $k>0$ and $(x)_{0}=1$ is the Pochhammer symbol. Note that

$$
\bar{Q}^{(n)}\left(y_{1}, y_{2}\right)=Q^{n}\left(y_{1}, y_{2}\right) \text { for } y_{1}-y_{2} \geqslant 1
$$

Using (2.8) and (2.21), we have

$$
Q^{-1} \bar{Q}^{(n)}=\bar{Q}^{(n)} Q^{-1}=\bar{Q}^{(n-1)} \text { for } n>1, \quad \text { but } \quad Q^{-1} \bar{Q}^{(1)}=\bar{Q}^{(1)} Q^{-1}=0 .
$$

Note also that $\bar{Q}^{(n)} \bar{Q}^{(m)}$ is divergent, so the $\bar{Q}^{(n)}$ are no longer a group like the $Q^{n}$. Let

$$
\tau=\min \left\{m \geqslant 0: B_{m}>X_{0}(m+1)\right\},
$$

where $B_{m}$ is now a random walk with transition matrix $Q$ (that is, $B_{m}$ has Geom $\left[\frac{1}{2}\right]$ jumps strictly in the negative direction). Using this stopping time and the extension of $Q^{m}$, we obtain the following result.

LEMmA 2.4. For the kernel defined in (2.17) and all $z_{1}, z_{2} \in \mathbb{Z}$, we have

$$
G_{0, n}\left(z_{1}, z_{2}\right)=\mathbb{E}_{B_{0}=z_{1}}\left[\bar{Q}^{(n-\tau)}\left(B_{\tau}, z_{2}\right) \mathbf{1}_{\tau<n}\right] .
$$

Proof. For $z_{2} \leqslant X_{0}(n),(2.20)$ can be written as

$$
\begin{aligned}
G_{0, n}\left(z_{1}, z_{2}\right) & =\mathbb{P}_{B_{-1}^{*}=z_{2}}\left(\tau^{0, n} \leqslant n-1, B_{n-1}^{*}=z_{1}\right) \\
& =\mathbb{P}_{B_{0}=z_{1}}\left(\tau \leqslant n-1, B_{n}=z_{2}\right) \\
& =\sum_{k=0}^{n-1} \sum_{z>X_{0}(k+1)} \mathbb{P}_{B_{0}=z_{1}}\left(\tau=k, B_{k}=z\right) Q^{n-k}\left(z, z_{2}\right) \\
& =\mathbb{E}_{B_{0}=z_{1}}\left[Q^{n-\tau}\left(B_{\tau}, z_{2}\right) \mathbf{1}_{\tau<n}\right] .
\end{aligned}
$$

We claim that the right-hand side of (2.25) equals $G_{0, n}\left(z_{1}, z_{2}\right)$ for all $z_{2} \leqslant X_{0}(n)$. To see this, note from the last equality in (2.26) that we only need to check that

$$
\chi_{X_{0}(k+1)} \bar{Q}^{(k+1)} \bar{\chi}_{X_{0}(n)}=\chi_{X_{0}(k+1)} Q^{k+1} \bar{\chi}_{X_{0}(n)} \quad \text { for } k=0, \ldots, n-1,
$$

which, since $X_{0}(k+1)-X_{0}(n) \geqslant n-k-1$, follows from $(2.22)$.

To complete the proof, recall that we showed that $2^{-z_{2}} h_{k}^{n}\left(0, z_{2}\right)$ is a polynomial of degree at most $k$ in $z_{2}$, so from $(2.17)$ we have that $2^{-z_{2}} G_{0, n}\left(z_{1}, z_{2}\right)$ satisfies the same (for every fixed $z_{1}$ ). It is straightforward to check that the right-hand side of (2.25) also satisfies this (because $\bar{Q}^{(m)}\left(z_{1}, z_{2}\right)$ does), and thus, since it coincides with $G_{0, n}\left(z_{1}, z_{2}\right)$ at infinitely many $z_{2}$ 's, we deduce the equality in (2.25). 


\subsection{Formulas for TASEP with right-finite initial data}

Let, $\left({ }^{9}\right)$ for $n \geqslant 1$,

$$
\begin{aligned}
\mathcal{S}_{-t,-n}\left(z_{1}, z_{2}\right) & =\left(e^{-(t / 2) \nabla^{-}} Q^{-n}\right)^{*}\left(z_{1}, z_{2}\right)=\frac{1}{2 \pi i} \oint_{\Gamma_{0}} d w \frac{(1-w)^{n}}{2^{z_{2}-z_{1}} w^{n+1+z_{2}-z_{1}}} e^{t(w-1 / 2)}, \\
\overline{\mathcal{S}}_{-t, n}\left(z_{1}, z_{2}\right) & =\bar{Q}^{(n)} e^{(t / 2) \nabla^{-}}\left(z_{1}, z_{2}\right)=\frac{1}{2 \pi i} \oint_{\Gamma_{0}} d w \frac{(1-w)^{z_{2}-z_{1}+n-1}}{2^{z_{1}-z_{2}} w^{n}} e^{t(w-1 / 2)}
\end{aligned}
$$

the contour integral formulas come from (2.8), (2.10), and (2.21). As before, $\Gamma_{0}$ is a simple counterclockwise loop around 0 not enclosing 1 . Define also, for $n \geqslant 0$,

$$
\overline{\mathcal{S}}_{-t, n}^{\mathrm{epi}\left(X_{0}\right)}\left(z_{1}, z_{2}\right)=\mathbb{E}_{B_{0}=z_{1}}\left[\overline{\mathcal{S}}_{-t, n-\tau}\left(B_{\tau}, z_{2}\right) \mathbf{1}_{\tau<n}\right]
$$

The superscript epi $\left(X_{0}\right)$ refers to the fact that $\tau$ (defined in (2.24)) is the hitting time of the strict epigraph $\left({ }^{10}\right)$ of the curve $\left(X_{0}(k+1)\right)_{k=0, \ldots, n-1}$ by the random walk $B_{k}$.

Remark 2.5. $M_{m}=\overline{\mathcal{S}}_{-t, n-m}\left(B_{m}, z_{2}\right)$ is not a martingale, because $Q \bar{Q}^{(n)}$ is divergent. So one cannot apply the optional stopping theorem to evaluate (2.29). The right-hand side of (2.29) is only finite because the curve $\left(X_{0}(k+1)\right)_{k=0, \ldots, n-1}$ cuts off the divergent sum.

We are now in position to state the general solution of TASEP with right-finite initial data.

Theorem 2.6. (TASEP formula for right-finite initial data) Assume that TASEP initial condition $X_{0}$ satisfies $X_{0}(j)=\infty$ for all $j \leqslant 0$. Then, for any distinct positive integers $n_{1}, \ldots, n_{m}$ and $t \geqslant 0$,

$$
\mathbb{P}\left(X_{t}\left(n_{j}\right)>a_{j}, j=1, \ldots, m\right)=\operatorname{det}\left(I-\bar{\chi}_{a} K_{t}^{\mathrm{TASEP}} \bar{\chi}_{a}\right)_{\ell^{2}\left(\left\{n_{1}, \ldots, n_{m}\right\} \times \mathbb{Z}\right)},
$$

where $K_{t}^{\mathrm{TASEP}}$ is the operator on $\ell^{2}\left(\left\{n_{1}, \ldots, n_{m}\right\} \times \mathbb{Z}\right)$ with kernel given by

$$
K_{t}^{\mathrm{TASEP}}\left(n_{i}, \cdot ; n_{j}, \cdot\right)=-Q^{n_{j}-n_{i}} \mathbf{1}_{n_{i}<n_{j}}+\left(\mathcal{S}_{-t,-n_{i}}\right)^{*} \overline{\mathcal{S}}_{-t, n_{j}}^{\mathrm{epi}\left(X_{0}\right)} .
$$

The path integral version $(2.12)$ (with $\left.K_{t}^{(n)}=K_{t}^{\mathrm{TASEP}}(n, \cdot ; n, \cdot)\right)$ also holds.

$\left({ }^{9}\right)\left(\overline{\mathcal{S}}_{-t, n}\right)^{*}$ should be thought of as a version of $\mathcal{S}_{-t, n}$ (analytic in $z_{2}-z_{1}$ ) made from the other pole in the contour integral in (2.3). In fact, if one changes variables $w \longmapsto 1-w$ in (2.27), and then changes $n$ to $-n$ then one gets (2.28) (with $z_{1}$ and $z_{2}$ interchanged), except that the integration is along a loop enclosing only 1 instead of only 0 . Our choice of taking an adjoint in the definition in (2.27) is made just for later convenience.

$\left({ }^{10}\right)$ The strict epigraph of a discrete curve $(g(m))_{m \geqslant 0}$ is the set epi $(g)=\{(m, y): m \geqslant 0$ and $y>g(m)\}$ (see also §3.1). 
Remark 2.7. (1) By shifting the indices of the particles, the theorem allows us to write a formula for any right-finite initial data $X_{0}$ with $X_{0}(j)=\infty$ for $j \leqslant \ell$, any $\ell \in \mathbb{Z}$. In fact, defining the shift operator

$$
\theta_{\ell} g(u)=g(u+\ell)
$$

we have the trivial identity

$$
\mathbb{P}_{X_{0}}\left(X_{t}\left(n_{j}\right)>a_{j} \text { for } j=1, \ldots, m\right)=\mathbb{P}_{\theta_{\ell} X_{0}}\left(X_{t}\left(n_{j}-\ell\right)>a_{j} \text { for } j=1, \ldots, m\right) \text {. }
$$

(2) Note that, by definition, $\overline{\mathcal{S}}_{-t, n_{j}}^{\mathrm{epi}\left(X_{0}\right)}(y, z)=\overline{\mathcal{S}}_{-t, n_{j}}(y, z)$ for $y>X_{0}(1)$, so (2.31) can also be written as

$$
\begin{aligned}
& K_{t}^{\mathrm{TASEP}}\left(n_{i}, \cdot ; n_{j}, \cdot\right) \\
& \quad=-Q^{n_{j}-n_{i}} \mathbf{1}_{n_{i}<n_{j}}+\left(\mathcal{S}_{-t,-n_{i}}\right)^{*} \chi_{X_{0}(1)} \overline{\mathcal{S}}_{-t, n_{j}}+\left(\mathcal{S}_{-t,-n_{i}}\right)^{*} \bar{\chi}_{X_{0}(1)} \overline{\mathcal{S}}_{-t, n_{j}}^{\mathrm{epi}\left(X_{0}\right)} .
\end{aligned}
$$

Proof. Consider first right-finite initial data. If $X_{0}(1)<\infty$ then we are in the setting of the above sections and formulas (2.30) and (2.31) follow directly from the above definitions together with (2.18) and Lemma 2.4. If $X_{0}(i)=\infty$ for $i=1, \ldots, \ell$ and $X_{0}(\ell+1)<$ $\infty$, then it is enough to consider $n_{j}>\ell$ for $j=1, \ldots, m$, and then, from (2.33), we have

$$
\mathbb{P}_{X_{0}}\left(X_{t}\left(n_{j}\right)>a_{j} \text { for } j=1, \ldots, m\right)=\operatorname{det}\left(I-\bar{\chi}_{a} K_{t}^{(\ell)} \bar{\chi}_{a}\right)_{\ell^{2}\left(\left\{n_{1}, \ldots, n_{m}\right\} \times \mathbb{Z}\right)},
$$

with

$$
K_{t}^{(\ell)}\left(n_{i}, \cdot ; n_{j}, \cdot\right)=-Q^{n_{j}-n_{i}} \mathbf{1}_{n_{i}<n_{j}}+\left(\mathcal{S}_{-t,-n_{i}+\ell}\right)^{*} \overline{\mathcal{S}}_{-t, n_{j}-\ell}^{\mathrm{epi}\left(\theta_{\ell} X_{0}\right)}
$$

Using now that, for $n_{i}, n_{j}>\ell,\left(\mathcal{S}_{-t,-n_{i}+\ell}\right)^{*}=\left(\mathcal{S}_{-t,-n_{i}}\right)^{*} Q^{\ell}$ and

$$
Q^{\ell} \overline{\mathcal{S}}_{-t, n_{j}-\ell}^{\mathrm{epi}\left(\theta_{\ell} X_{0}\right)}=\overline{\mathcal{S}}_{-t, n_{j}}^{\mathrm{epi}\left(X_{0}\right)}
$$

which follows from the definition of $\overline{\mathcal{S}}_{-t, n}^{\mathrm{epi}\left(X_{0}\right)},(2.23)$, and the fact that $\theta_{\ell} X_{0}(j)=\infty$ for $j=1, \ldots, \ell$, we see that $(2.31)$ still holds in this case.

The path integral formula (2.12) is proved in Appendix D.2, and follows from a variant of [8, Theorem 3.3] proved in Appendix D.1.

Example 2.8. (Step initial data) Consider TASEP with step initial data, i.e.

$$
X_{0}(i)=-i \text { for } i \geqslant 1 \text {. }
$$

If we start the random walk in (2.29) from $B_{0}=z_{1}$ below the curve, i.e. $z_{1} \leqslant-1$, then the random walk clearly never hits the epigraph. Hence, $\bar{\chi}_{X_{0}(1)} \overline{\mathcal{S}}_{-t, n}^{\mathrm{epi}\left(X_{0}\right)} \equiv 0$ and the last term in (2.34) vanishes. For the second term in (2.34) we have, from (2.27) and (2.28),

$$
\left(\mathcal{S}_{-t,-n_{i}}\right)^{*} \chi_{X_{0}(1)} \overline{\mathcal{S}}_{-t, n_{j}}\left(z_{1}, z_{2}\right)=\frac{1}{(2 \pi i)^{2}} \oint_{\Gamma_{0}} d w \oint_{\Gamma_{0}} d v \frac{(1-w)^{n_{i}}(1-v)^{n_{j}+z_{2}}}{2^{z_{1}-z_{2}} w^{n_{i}+z_{1}+1} v^{n_{j}}} \frac{e^{(w+v-1)}}{1-v-w} .
$$

Using this in (2.34) yields exactly the formula derived previously in the literature (see e.g. [24, equation 82]), modulo the conjugation by $2^{z_{2}-z_{1}}$. 
Example 2.9. (2-periodic initial data) We are interested now in TASEP with 2periodic initial data $X_{0}(i)=2 i, i \in \mathbb{Z}$ (we consider more general periods in the next example). To obtain a formula for the kernel in this case we will approximate by considering first the finite periodic initial data $X_{0}(i)=2(N-i)$ for $i=1, \ldots, 2 N$. For simplicity, we will compute only $K_{t}^{(n)}=K^{\operatorname{TASEP}}(n, \cdot ; n, \cdot)$.

We start by computing $\overline{\mathcal{S}}_{-t, n}^{\mathrm{epi}\left(X_{0}\right)}$. Observe that, for $\lambda>-\log (2)$, we have that

$$
\left(e^{\lambda B_{m}-m \varphi(\lambda)}\right)_{m \geqslant 0},
$$

with $\varphi(\lambda)=-\log \left(2 e^{\lambda}-1\right)$ being the logarithm of the moment generating function of a negative Geom $\left[\frac{1}{2}\right]$ random variable, is a martingale. Thus, if $z \leqslant 2(N-1)$, we have

$$
\mathbb{E}_{B_{0}=z}\left[e^{\lambda B_{\tau}-\tau \varphi(\lambda)}\right]=e^{\lambda z}
$$

But it is easy to see from the definition of $X_{0}$ that, if the walk starts below the curve, then $B_{\tau}$ is necessarily $2(N-(\tau+1))+1$, so we have

$$
\mathbb{E}_{B_{0}=z}\left[e^{-2 \lambda \tau}\left(2 e^{\lambda}-1\right)^{\tau}\right]=e^{(z-2 N+1) \lambda}
$$

Introducing a new variable $\eta \in(0,1)$ through $\lambda=\log \left(\eta^{-1}(1+\sqrt{1-\eta})\right)$ yields

$$
\mathbb{E}_{B_{0}=z}\left[\eta^{\tau}\right]=\left(\eta^{-1}(1+\sqrt{1-\eta})\right)^{z-2 N+1}
$$

As a consequence, we obtain

$$
\mathbb{P}_{B_{0}=z}(\tau=k)=\lim _{\eta \rightarrow 0} \frac{1}{k !} \frac{d^{k}}{d \eta^{k}}\left(\frac{1+\sqrt{1-\eta}}{\eta}\right)^{z-2 N+1}
$$

and then using Cauchy's integral formula we can compute $\overline{\mathcal{S}}_{-t, n}^{\mathrm{epi}\left(X_{0}\right)}\left(z_{1}, z_{2}\right)$ from $(2.28)$ and (2.29) as

$$
\lim _{\eta \rightarrow 0} \frac{1}{(2 \pi i)^{2}} \oint_{\gamma_{r}} d w \oint_{\eta+\gamma_{r}} d \xi \sum_{k=0}^{n-1}\left(\frac{1+\sqrt{1-\xi}}{\xi}\right)^{z_{1}-2 N+1} \frac{1}{(\xi-\eta)^{k+1}} \frac{(1-w)^{z_{2}-2 N+n+k}}{2^{2(N-k)-1-z_{2}} w^{n-k}} e^{t(w-1 / 2)}
$$

where $\gamma_{r}$ is a circle of radius $r$ centered at the origin and we take $\eta<r<\frac{3}{4}$. The $\eta \rightarrow 0$ limit is now straightforward to compute, and since the resulting integrand is analytic in $\xi$ for $k<0$, we may extend the sum to $k=-\infty$, and then compute the sum (using that $\left|4 \xi^{-1} w(1-w)\right|>1$ for our choice of $\left.r\right)$ to get

$$
\frac{1}{(2 \pi i)^{2}} \oint_{\gamma_{r}} d w \oint_{\gamma_{r}} d \xi\left(\frac{1+\sqrt{1-\xi}}{\xi}\right)^{z_{1}-2 N+1} \frac{(1-w)^{z_{2}-2(N-n)}}{2^{2(N-n)-1-z_{2} \xi^{n}}} \frac{1}{4 w(1-w)-\xi} e^{t(w-1 / 2)}
$$


Now, we introduce the change of variables $\xi=4 v(1-v)$, which is locally 1-to-one near $v=1$. For small $r$, if $v$ lies in $1+\gamma_{r}$, then $4 v(1-v)$ lies approximately in $\gamma_{4 r}$, so we may adjust the contours to get that the last integral equals

$$
\frac{1}{(2 \pi i)^{2}} \oint_{\gamma_{r}} d w \oint_{1+\gamma_{r^{\prime}}} d v \frac{(1-w)^{z_{2}-2(N-n)}}{2^{y-z_{2}} v^{n}(1-v)^{z_{1}-2 N+n+1}} \frac{1-2 v}{w(1-w)-v(1-v)} e^{t(w-1 / 2)},
$$

with $r^{\prime} \sim \frac{1}{4} r$. From this and (2.27), we may compute the product

$$
\left(\mathcal{S}_{t,-n}\right)^{*} \bar{\chi}_{2(N-1)} \overline{\mathcal{S}}_{t, n}^{\mathrm{epi}\left(X_{0}\right), \eta}\left(z_{1}, z_{2}\right),
$$

which equals

$$
\begin{aligned}
\frac{1}{(2 \pi i)^{3}} \oint_{\gamma_{r}} d u \oint_{\gamma_{r}} d w \oint_{1+\gamma_{r^{\prime}}} d v & \frac{(1-w)^{z_{2}-2(N-n)}(1-u)^{n}}{2^{z_{1}-z_{2}} v^{n}(1-v)^{n-1} u^{z_{1}-2 N+n+2}} \\
& \times \frac{1-2 v}{(1-u-v)[w(1-w)-v(1-v)]} e^{t(w+u-1)} .
\end{aligned}
$$

Since $1-v$ lies inside $\gamma_{r}$, the $w$ integral has a pole at $w=1-v$, and computing the residue yields

$$
\frac{1}{(2 \pi i)^{2}} \oint_{\gamma_{r}} d u \oint_{1+\gamma_{r^{\prime}}} d v \frac{v^{z_{2}-2 N+n}(1-u)^{n}}{2^{z_{1}-z_{2}}(1-v)^{n-1} u^{z_{1}-2 N+n+2}} \frac{1}{1-u-v} e^{t(u-v)} .
$$

On the other hand, a simpler computation (as in the previous example) shows that the other term making up

$$
K_{t}^{(n)}\left(z_{1}, z_{2}\right)=K_{t}^{\mathrm{TASEP}}\left(n, z_{1} ; n, z_{2}\right)
$$

in $(2.34)$, namely $\left(\mathcal{S}_{t,-n}\right)^{*} \chi_{2(N-1)} \overline{\mathcal{S}}_{t, n}\left(z_{1}, z_{2}\right)$, equals

$$
\frac{1}{(2 \pi i)^{2}} \oint_{\gamma_{r}} d u \oint_{\gamma_{r^{\prime \prime}}} d v \frac{(1-v)^{z_{2}-2 N+n+1}(1-u)^{n}}{2^{z_{1}-z_{2}} u^{z_{1}-2 N+n+2} v^{n}} \frac{1}{1-u-v} e^{t(u+v-1)},
$$

where we take $r^{\prime \prime}<r$. Changing variables $v \mapsto 1-v$ in (2.36) and adding the result with (2.37), we get

$$
K_{t}^{(n)}\left(z_{1}, z_{2}\right)=\frac{1}{(2 \pi i)^{2}} \oint_{\gamma_{r}} d u \oint_{\gamma_{\gamma^{\prime \prime}}} d v \frac{(1-v)^{z_{2}-2 N+n}(1-u)^{n}}{2^{z_{1}-z_{2}} u^{z_{1}-2 N+n+1} v^{n}} \frac{1-2 v}{(u-v)(1-u-v)} e^{t(u+v-1)} .
$$

In order to obtain the kernel which yields the distribution of the $m$ th particle for the full 2-periodic initial condition, $K_{t}^{2 \text {-prd, }(m)}$, we proceed as in [10], using the last formula and focusing on particles which start at a fixed distance from the origin, that is $n=N+m$ with $m$ fixed (which corresponds to the particle that started at $-2 m$ ), and taking $N \rightarrow \infty$. 
To this end, for fixed $z_{1}$, take $N \geqslant z_{1}+m+2$, so that $u=0$ is not a pole in both (2.36) and (2.37). We see now that (2.37) vanishes, because the $u$ integrand is analytic, given our choice of contours. On the other hand, for (2.36) we have that $1-v$ lies inside $\gamma_{r}$ so the $u$ integral has a pole at $u=1-v$, and computing the residue yields

$$
K_{t}^{2-\operatorname{prd},(m)}\left(z_{1}, z_{2}\right)=-\frac{1}{2 \pi i} \oint_{1+\gamma_{r^{\prime}}} d v \frac{v^{z_{2}+2 m}}{2^{z_{1}-z_{2}}(1-v)^{z_{1}+2 m+1}} e^{t(1-2 v)} .
$$

This is exactly the kernel derived (modulo the conjugation $2^{z_{2}-z_{1}}$, and after a simple change of variables) in [10, Theorem 2.2].

Example 2.10. ( $\ell$-periodic initial data) Now, we turn to TASEP with $\ell$-periodic initial data, given by $X_{0}(i)=-\ell i, i \in \mathbb{Z}$, with $\ell \geqslant 2$. In contrast to the last example, the value of $B_{\tau}$ is not fixed as a function of $\tau$ if $\ell>2$, and thus computing $\overline{\mathcal{S}}_{t,-n}^{\text {epi }\left(X_{0}\right)}$ becomes more complicated. But the hitting probabilities for $B^{*}$ can be computed in a way similar to the last example, so in this case it is simpler to compute the biorthogonal functions $\Phi_{k}^{n}$ using (2.15) and (2.19), and then obtain the kernel $K_{t}^{(n)}=K_{t}^{\mathrm{TASEP}}(n, \cdot ; n, \cdot)$ directly from (2.6). We do this next.

As in the last example, we consider first the truncated initial data $X_{0}(i)=\ell(N-i)$, for $i=1, \ldots, 2 N$. For fixed $0 \leqslant k<n \leqslant 2 N$ and $z \leqslant X_{0}(1)$, we want to compute

$$
h_{k}^{n}(z)=\mathbb{P}_{B_{-1}^{*}=z}\left(\tau^{*}=k\right),
$$

where $\tau^{*}$ is the hitting time of the strict epigraph of $\left(X_{0}(n-m)\right)_{m=0, \ldots, n-1}$. Proceeding as above, for $\lambda<\log (2)$ and $\varphi^{*}(\lambda)=-\log \left(2 e^{-\lambda}-1\right)$, we have

$$
\mathbb{E}_{B_{-1}^{*}=z}\left[e^{\lambda B_{\tau^{*}}^{*}-\tau^{*} \varphi^{*}(\lambda)}\right]=e^{\lambda z+\varphi^{*}(\lambda)},
$$

giving

$$
e^{\lambda z+\varphi^{*}(\lambda)}=\sum_{k \geqslant 0} \sum_{m \geqslant 1} \mathbb{P}_{B_{-1}^{*}=z}\left(\tau^{*}=k, B_{k}^{*}=X_{0}(n-k)+m\right) e^{\lambda\left(X_{0}(n-k)+m\right)-k \varphi^{*}(\lambda)} .
$$

Using the memoryless property of the geometric distribution, we may rewrite the above probability as $\mathbb{P}_{B_{-1}^{*}=z}\left(\tau^{*}=k, B_{k}^{*}=X_{0}(n-k)+1\right) 2^{1-m}$. The sum over $m$ is then just

$$
\sum_{m \geqslant 1}\left(\frac{e^{\lambda}}{2}\right)^{m}=e^{\varphi^{*}(\lambda)},
$$

and thus the right-hand side equals

$$
2 \mathbb{E}_{B_{-1}^{*}=z}\left[e^{\lambda B_{\tau^{*}}^{*}-\tau^{*} \varphi^{*}(\lambda)} \mathbf{1}_{B_{\tau^{*}}^{*}=X_{0}\left(n-\tau^{*}\right)+1}\right] e^{\varphi^{*}(\lambda)-\lambda},
$$


which leads to

$$
2 \mathbb{E}_{B_{-1}^{*}=z}\left[e^{\left(\lambda \ell-\varphi^{*}(\lambda)\right) \tau^{*}} \mathbf{1}_{B_{\tau^{*}}^{*}=X_{0}\left(n-\tau^{*}\right)+1}\right]=e^{\lambda[z+\ell(n-N)]} .
$$

Setting $v=1-\frac{1}{2} e^{\lambda}>0$, we get

$$
2 \mathbb{E}_{B_{-1}^{*}=z}\left[\left(2^{\ell}(1-v)^{\ell-1} v\right)^{\tau^{*}} \mathbf{1}_{B_{\tau^{*}}^{*}=X_{0}\left(n-\tau^{*}\right)+1}\right]=(2(1-v))^{z+\ell(n-N)} .
$$

The function $v \mapsto p(v):=2^{\ell}(1-v)^{\ell-1} v$ is locally 1 -to-one near zero, so

$$
2 \mathbb{P}_{B_{-1}^{*}=z}\left(\tau^{*}=k, B_{\tau^{*}}^{*}=X_{0}\left(n-\tau^{*}\right)+1\right)=\lim _{v \searrow 0} \frac{1}{k !} \frac{d^{k}}{d p(v)^{k}}(2(1-v))^{z+\ell(n-N)} .
$$

Using again the memorylessness of the walk, the left-hand side equals $\mathbb{P}_{B_{-1}^{*}=z}\left(\tau^{*}=k\right)$, while, by Cauchy's formula, the right-hand side equals

$$
\frac{1}{2 \pi i} \oint_{\Gamma_{0}} d v \frac{1}{p(v)^{k+1}} p^{\prime}(v)(2(1-v))^{z+\ell(n-N)}=\frac{1}{2 \pi i} \oint_{\Gamma_{0}} d v \frac{(1-v)^{z+\ell(n-N)-1}}{2^{\ell(N-n+k)-z}\left((1-v)^{d-1} v\right)^{k}} \frac{(1-\ell v)}{v}
$$

where $\Gamma_{0}$ goes around 0 but not 1 . In principle, this is only valid for $z \leqslant X_{0}(1)$, but the right-hand side is analytic in $z$, so we actually get a formula for $h_{k}^{n}(0, z)$ for all $z \in \mathbb{Z}$. Using (2.10) and (2.15), we get

$$
\Phi_{k}^{n}(z)=\frac{1}{2 \pi i} \oint_{\Gamma_{0}} d v \frac{(1-v)^{z+\ell(n-N)-1}}{2^{\ell(N-n+k)-z}\left((1-v)^{\ell-1} v\right)^{k}} \frac{1-\ell v}{v} e^{t v}
$$

These functions extend trivially to 0 for $k<0$, so we may now perform the summation in (2.6) over $k \geqslant 1$ (using the explicit formula (2.7) for $\Psi_{k}^{n}$ ) to get the kernel for truncated $\ell$-periodic initial data

$$
K_{t, N}^{(n)}\left(z_{1}, z_{2}\right)=\frac{1}{(2 \pi i)^{2}} \oint_{\Gamma_{0}} d w \oint_{\Gamma_{0}^{\prime}} d v \frac{(1-v)^{z_{2}-\ell(N-1)+n-2}(1-w)^{n}}{2^{z_{1}-z_{2}} v^{n} w^{z_{1}-\ell N+n+1}} \frac{(1-\ell v) e^{t(v+w-1)}}{(1-w) w^{\ell-1}-(1-v)^{\ell-1} v},
$$

where the contours are such that

$$
\left|(1-v)^{\ell-1} v\right|<\left|(1-w) w^{\ell-1}\right|
$$

Finally, and as in the 2-periodic case, we set $n=N+m$ in the last kernel, which gives

$$
\frac{1}{(2 \pi i)^{2}} \oint_{\Gamma_{-1}} d w \oint_{\Gamma_{0}} d v \frac{(1+v)^{z_{2}-(\ell-1) N+\ell+m-2} w^{N+m}}{2^{z_{1}-z_{2}} v^{N+m}(1+w)^{z_{1}-(\ell-1) N+m+1}} \frac{(1+\ell v) e^{t(w-v)}}{(1+w)^{\ell-1} w-(1+v)^{\ell-1} v}
$$


(where we have changed variables $v \mapsto-v$ and $w \mapsto 1+w$ ), and then take $N \rightarrow \infty$ to get the kernel for the full $\ell$-periodic initial condition. For fixed $z_{1}$ and large enough $N$, the $w$ integral has no pole at -1 . Let $w_{1}(v), \ldots w_{\ell-1}(v)$ be the $\ell-1$ solutions of

$$
(1+w)^{\ell-1} w=(1+v)^{\ell-1} v
$$

other than $w=v$. One can check that all these $\ell-1$ roots are distinct and lie inside the $w$ contour, while $w=v$ lies outside of it. The full $\ell$-periodic kernel then evaluates to a sum over the residues of these $\ell-1$ simple poles, and after simplification (using the equation satisfied by the $w_{\ell}(v)$ 's) we get

$$
\begin{aligned}
& K_{t}^{\ell-\operatorname{prd},(m)}\left(z_{1}, z_{2}\right)= \frac{1}{2 \pi i} \oint_{\Gamma_{0}} d v \sum_{j=1}^{\ell-1} \frac{1+\ell v}{1+\ell w_{j}(v)} \frac{(1+v)^{z_{2}+\ell+m-2} w_{j}(v)^{m}}{2^{z_{1}-z_{2}} v^{m}\left(1+w_{j}(v)\right)^{z_{1}+\ell+m-1}} e^{t\left(w_{j}(v)-v\right)} \\
&=\frac{1}{(2 \pi i)^{2}} \oint_{\Gamma_{-1}} d w \oint_{\Gamma_{0}} d v \frac{(1+v)^{z_{2}+\ell+m-2} w^{m}}{2^{z_{1}-z_{2}} v^{m}(1+w)^{z_{1}+m+1}} \\
& \times \frac{1+\ell v}{(1+w)^{\ell-1} w-(1+v)^{\ell-1} v} e^{t(w-v)} .
\end{aligned}
$$

These formulas are very similar to [9, equations 2.3 and 4.11] (which are for discrete-time TASEP). In the case $\ell=2$, we have $w_{1}(v)=-1-v$ and we recover (2.38) after a simple change of variables.

\subsection{Integrability}

There are many notions of classical integrable systems: Liouville integrability, algebraic integrability, etc. Quantum integrability usually is used to mean that a quantum mechanical model possesses an infinite number of conserved quantities. Another notion of integrable system is simply that one has a representation under which the flow is linearized. Theorem 2.6 presents TASEP with right-finite initial data $\left({ }^{11}\right)$ as a new type of stochastic integrable system, the dynamics being trivialized at the level of kernels, which satisfy the Lax equation, $\left({ }^{12}\right)$

$$
\partial_{t} K_{t}^{\mathrm{TASEP}}=\frac{1}{2}\left[K_{t}^{\mathrm{TASEP}}, \nabla^{-}\right] .
$$

(11) One also has a formula for 2-sided initial data, but because of the analytic extension it is cumbersome, and the proof is quite lengthy; moreover, it is not clear to us yet that the formula can be used for asymptotics. We leave it to a future paper.

$\left.{ }^{12}\right) K_{t}^{\text {TASEP }}$ acts on the Hilbert space $\ell^{2}\left(\left\{n_{1}, \ldots, n_{m}\right\} \times \mathbb{Z}\right)$, and can be identified with an operatorvalued $n \times n$ matrix acting on

$$
\bigoplus_{n \in\left\{n_{1}, \ldots, n_{m}\right\}} \ell^{2}(\mathbb{Z}) .
$$

Under this identification, $\nabla^{-}$is identified with the diagonal matrix with the specified entries along the whole diagonal. 
The $m$-point distributions at time $t$ are obtained from $K_{t}^{\text {TASEP }}$ by projecting down via the Fredholm determinant, and the full space-time field is recovered from these transition probabilities using the Markov property.

TASEP has long been known to be solvable, by the coordinate Bethe ansatz, resulting in Schütz's formula (2.2). One also has the algebraic Bethe ansatz, in which the eigenfunctions are computable [46]. However, the resulting formulas do not directly integrate the dynamics - i.e., solve the problem starting from generic initial data - in a useful way. One might refer to them as exact solvability versus the stochastic integrability given in (2.39).

\section{1:2:3 scaling limit}

For each $\varepsilon>0$, the 1:2:3 rescaled TASEP height function is

$$
\mathfrak{h}^{\varepsilon}(\mathbf{t}, \mathbf{x})=\varepsilon^{1 / 2}\left[h_{2 \varepsilon^{-3 / 2} \mathbf{t}}\left(2 \varepsilon^{-1} \mathbf{x}\right)+\varepsilon^{-3 / 2} \mathbf{t}\right] .
$$

Remark 3.1. The KPZ fixed point has one free parameter, $\left({ }^{13}\right)$ corresponding to $\lambda$ in (1.2). Our choice of the height function in TASEP moving downwards corresponds to $\lambda>0 .\left({ }^{14}\right)$ The scaling of space and time by the factor 2 in (3.1) corresponds to the choice $|\lambda|=\frac{1}{4}$.

Note also that, for fixed the the TASEP height function in (3.1) is being rescaled diffusively in space. In particular, this fixes our study of the scaling limit to perturbations of density $\frac{1}{2}$. We could perturb off any density $\rho \in(0,1)$ without extra difficulty by observing the system in an appropriate moving frame, but in order to avoid heavier notation we do not pursue it here.

Assume that we have initial data $X_{0}^{\varepsilon}$ chosen to depend on $\varepsilon$ in such a way that

$$
\mathfrak{h}_{0}=\lim _{\varepsilon \rightarrow 0} \mathfrak{h}^{\varepsilon}(0, \cdot)
$$

$\left({ }^{13}\right)$ It was recently proposed that the KPZ fixed point is given by

$$
\partial_{t} h=\lambda\left(\partial_{x} h\right)^{2}-\nu\left(-\partial_{x}^{2}\right)^{3 / 2} h+\nu^{1 / 2}\left(-\partial_{x}^{2}\right)^{3 / 4} \xi, \quad \nu>0,
$$

the evidence being that formally it is invariant under the 1:2:3 KPZ scaling (1.1), and it preserves Brownian motion. Besides the non-physical non-locality, and the inherent difficulty of making sense of this equation, one can see that it is not correct, because it has two free parameters instead of one. Presumably, it converges to the KPZ fixed point in the limit $\nu \searrow 0$. On the other hand, the model has critical scaling, so it is also plausible that, if one introduces a cutoff (say, smooth the noise) and then take a limit, the result has $\nu=0$, and possibly even a renormalized $\lambda$. So, it is possible that, in a rather uninformative sense, the conjecture could still be true.

$\left({ }^{14}\right)$ To get some intuition, check that $-\mathbf{x}^{2} / \mathbf{t}$ is a solution of $\partial_{t} \mathfrak{h}=\frac{1}{4}\left(\partial_{x} \mathfrak{h}\right)^{2}$; it corresponds to step initial data. The bulk downward movement of the TASEP height function has been compensated by the huge shift upward in (3.1). 
in distribution, in the UC topology described below. We will also choose the frame of reference

$$
X_{0}^{-1}(-1)=1,
$$

i.e. the particle labeled 1 is initially the rightmost in $\mathbb{Z}_{<0}$. Because the $X_{0}^{\varepsilon}(k)$ are in reverse order, and because of (3.3) and the inversion (2.1), we have that (3.2) is equivalent to

$$
\varepsilon^{1 / 2}\left(X_{0}^{\varepsilon}\left(\varepsilon^{-1} \mathbf{x}\right)+2 \varepsilon^{-1} \mathbf{x}-1\right) \underset{\varepsilon \rightarrow 0}{\longrightarrow}-\mathfrak{h}_{0}(-\mathbf{x})
$$

in distribution, in UC, where the left-hand side is interpreted as a linear interpolation to make it a continuous function of $\mathbf{x} \in \mathbb{R}$.

For fixed $\mathbf{t}>0$, we will now show that the limit

$$
\mathfrak{h}\left(\mathbf{t}, \mathbf{x} ; \mathfrak{h}_{0}\right)=\lim _{\varepsilon \rightarrow 0} \mathfrak{h}^{\varepsilon}(\mathbf{t}, \mathbf{x})
$$

also exists in distribution, in UC. In $\S 3.5$ we will prove the Markov property, which gives us, in principle, the multi-time and space distributions of the entire field. We take (3.5) essentially as our definition of the KPZ fixed point $\mathfrak{h}\left(\mathbf{t}, \mathbf{x} ; \mathfrak{h}_{0}\right)$. We will often omit $\mathfrak{h}_{0}$ from the notation, when it is clear from the context.

\subsection{State space and topology}

The state space in which we will always work, and where (3.2) and (3.4) will be assumed to hold and (3.5) will be proved, in distribution, will be $\left({ }^{15}\right)$ the set

$$
\begin{aligned}
& \mathrm{UC}=\{\mathfrak{h}: \mathbb{R} \rightarrow w[-\infty, \infty) \text { such that } \mathfrak{h} \text { is upper semicontinuous and } \\
& \qquad \mathfrak{h}(\mathbf{x}) \leqslant \overline{\boldsymbol{\alpha}}+\bar{\gamma}|\mathbf{x}| \text { for some } \overline{\boldsymbol{\alpha}}, \bar{\gamma}<\infty \text { and } \mathfrak{h}(\mathbf{x})>-\infty \text { for some } \mathbf{x}\},
\end{aligned}
$$

with the topology of local UC convergence, which we now describe.

Recall that $\mathfrak{h}$ is upper semicontinuous (UC) if and only if its hypograph

$$
\operatorname{hypo}(\mathfrak{h})=\{(\mathbf{x}, \mathbf{y}): \mathbf{y} \leqslant \mathfrak{h}(\mathbf{x})\}
$$

is closed in $[-\infty, \infty) \times \mathbb{R}$. We endow $[-\infty, \infty)$ with the distance $\left({ }^{16}\right)$

$$
d_{[-\infty, \infty)}\left(\mathbf{y}_{1}, \mathbf{y}_{2}\right)=\left|e^{\mathbf{y}_{1}}-e^{\mathbf{y}_{2}}\right| .
$$

$\left.{ }^{15}\right)$ The bound $\mathfrak{h}(\mathbf{x}) \leqslant \overline{\boldsymbol{\alpha}}+\overline{\boldsymbol{\gamma}}|\mathbf{x}|$ is not as general as possible. With work, one can extend to the class $\mathfrak{h}(\mathbf{x}) \leqslant \overline{\boldsymbol{\alpha}}+\overline{\boldsymbol{\gamma}}_{0}|\mathbf{x}|^{2}$, up to time $\mathbf{t}=\overline{\boldsymbol{\gamma}}_{0}^{-1}$. At that time, initial data such as $\mathfrak{h}_{0}(\mathbf{x})=\overline{\boldsymbol{\alpha}}+\overline{\boldsymbol{\gamma}}_{0}|\mathbf{x}|^{2}$ will actually have an explosion.

$\left({ }^{16}\right)$ This allows continuity at time zero for initial data which takes values $-\infty$, such as half-flat (see $\S 4.4)$. 
Given $\mathfrak{h}_{1}, \mathfrak{h}_{2} \in \mathrm{UC}$ and $M>0$, we say that the hypographs $\mathfrak{H}_{1}^{M}$ of $\mathfrak{h}_{1}$ and $\mathfrak{H}_{2}^{M}$ of $\mathfrak{h}_{2}$ restricted to $[-M, M]$ are $\delta$-close if $B_{\delta}\left(\mathfrak{H}_{1}^{M}\right) \subseteq \mathfrak{H}_{2}^{M}$ and $B_{\delta}\left(\mathfrak{H}_{2}^{M}\right) \subseteq \mathfrak{H}_{1}^{M}$, where

$$
B_{\delta}(\mathfrak{H}):=\bigcup_{(\mathbf{t}, \mathbf{x}) \in \mathfrak{H}} B_{\delta}((\mathbf{t}, \mathbf{x})),
$$

$B_{\delta}((\mathbf{t}, \mathbf{x}))$ being the ball of radius $\delta$ around $(\mathbf{t}, \mathbf{x})$, i.e. we use the Hausdorff distance on the restricted hypographs. We say then that $\left(\mathfrak{h}_{\varepsilon}\right)_{\varepsilon} \subseteq \mathrm{UC}$ converges locally in UC to $\mathfrak{h} \in \mathrm{UC}$ if there is a $\bar{\gamma}>0$ such that $\mathfrak{h}_{\varepsilon}(\mathbf{x}) \leqslant \overline{\boldsymbol{\alpha}}+\bar{\gamma}|\mathbf{x}|$ for all $\varepsilon>0$ and for every $M \geqslant 1$ there is a $\delta=\delta(\varepsilon, M)>0$ going to 0 as $\varepsilon \rightarrow 0$ such that the hypographs $\mathfrak{H}_{\varepsilon}^{M}$ of $\mathfrak{h}_{\varepsilon}$ and $\mathfrak{H}^{M}$ of $\mathfrak{h}$ restricted to $[-M, M]$ are $\delta$-close.

Another characterization is that $\mathfrak{h}^{n} \rightarrow \mathfrak{h}$ locally in UC if and only if, for each $\mathbf{x}$,

$$
\limsup _{\mathbf{x}^{n} \rightarrow \mathbf{x}} \mathfrak{h}^{n}\left(\mathbf{x}^{n}\right) \leqslant \mathfrak{h}(\mathbf{x})
$$

and there exists $\mathbf{x}^{n} \rightarrow \mathbf{x}$ with

$$
\liminf _{\mathbf{x}^{n} \rightarrow \mathbf{x}} \mathfrak{h}^{n}\left(\mathbf{x}^{n}\right) \geqslant \mathfrak{h}(\mathbf{x}) .
$$

We will also use the space $\mathrm{LC}=\{\mathfrak{g}:-\mathfrak{g} \in \mathrm{UC}\}$ (made of lower semicontinuous functions), the topology now being defined in terms of epigraphs,

$$
\operatorname{epi}(\mathfrak{g})=\{(\mathbf{x}, \mathbf{y}): \mathbf{y} \geqslant \mathfrak{g}(\mathbf{x})\}
$$

The Borel sets of $\mathrm{UC}$ will be denoted $\mathcal{B}(\mathrm{UC})$. It is fairly easy to see that $\mathrm{UC}$ is a Polish space and the subspace $\mathcal{B}_{0}(\mathrm{UC}) \subseteq \mathcal{B}(\mathrm{UC})$ of sets $A$ of the form

$$
A=\left\{\mathfrak{h} \in \mathrm{UC}: \mathfrak{h}\left(\mathbf{x}_{i}\right) \leqslant \mathbf{a}_{i} \text { for } i=1, \ldots, n\right\}
$$

defines a generating family for the $\sigma$-algebra $\mathcal{B}(\mathrm{UC})$, as does the subspace $\mathcal{B}_{1}(\mathrm{UC}) \subseteq$ $\mathcal{B}(\mathrm{UC})$ of sets of the type

$$
A_{\mathfrak{g}}=\{\mathfrak{h} \in \mathrm{UC}: \mathfrak{h}(\mathbf{x}) \leqslant \mathfrak{g}(\mathbf{x}) \text { for } \mathbf{x} \in \mathbb{R}\}, \quad \mathfrak{g} \in \mathrm{LC}
$$

The UC topology is very natural for interface growth, incorporating the inherent lateral growth mechanism and the $\mathfrak{h} \rightarrow-\mathfrak{h}$ asymmetry.

One could alternatively consider the set $\mathscr{C}_{\bar{\gamma}}$ of continuous functions in UC satisfying

$$
\mathfrak{h}(\mathbf{x}) \leqslant \overline{\boldsymbol{\alpha}}+\bar{\gamma}|\mathbf{x}|
$$

and use

$$
\mathscr{C}=\bigcup_{\bar{\gamma}>0} \mathscr{C}_{\bar{\gamma}}
$$


as state space; the topology on $\mathrm{UC}$, when restricted to $\mathscr{C}$, is the topology of uniform convergence on compact sets. Or we could consider the local Hölder spaces

$$
\mathscr{C}_{\bar{\gamma}}^{\beta}=\left\{\mathfrak{h} \in \mathscr{C}_{\bar{\gamma}}:\|\mathfrak{h}\|_{\beta,[-M, M]}<\infty \text { for each } M=1,2, \ldots\right\},
$$

defined by the family of semi-norms

$$
\|\mathfrak{h}\|_{\beta,[-M, M]}=\sup _{\mathbf{x}_{1} \neq \mathbf{x}_{2} \in[-M, M]} \frac{\left|\mathfrak{h}\left(\mathbf{x}_{2}\right)-\mathfrak{h}\left(\mathbf{x}_{1}\right)\right|}{\left|\mathbf{x}_{2}-\mathbf{x}_{1}\right|^{\beta}} .
$$

These would suffice for any $\mathbf{t}>0$, but many natural initial data are not in these spaces. For example, the UC function $\mathfrak{d}_{\mathbf{x}}(\mathbf{x})=0, \mathfrak{d}_{\mathbf{x}}(\mathbf{y})=-\infty$ for $\mathbf{y} \neq \mathbf{x}$, known as a narrow wedge at $\mathbf{x}$, plays a role in the theory somewhat analogous to Dirac's delta function.

For our purposes, the following fact about UC is crucial. Recall that $\boldsymbol{\tau}_{\varepsilon} \rightarrow \boldsymbol{\tau}$ in distribution if and only if $\limsup \boldsymbol{\tau}_{\varepsilon} \leqslant \boldsymbol{\tau}$ and $\liminf \boldsymbol{\tau}_{\varepsilon} \geqslant \boldsymbol{\tau}$ in distribution, i.e.

$$
\limsup \mathbb{P}\left(\boldsymbol{\tau}_{\varepsilon} \geqslant r\right) \leqslant \mathbb{P}(\boldsymbol{\tau} \geqslant r) \text { and } \liminf \mathbb{P}\left(\boldsymbol{\tau}_{\varepsilon}>r\right) \geqslant \mathbb{P}(\boldsymbol{\tau}>r) .
$$

The probability spaces on which they are defined need have nothing to do with each other, but it can be conceptually easier to construct them all on the same probability space, in which case the definitions are just the standard ones.

Proposition 3.2. Suppos $\mathfrak{g}_{\varepsilon} \rightarrow \mathfrak{g}$ locally in LC. Let $\mathbf{B}(\mathbf{x}), \mathbf{x} \geqslant 0$, be a Brownian motion starting at $z<\mathfrak{g}(0)$, and let $\mathbf{B}_{\varepsilon}(\mathbf{x})$ be stochastic processes with $\mathbf{B}_{\varepsilon} \rightarrow \mathbf{B}$ in $\mathrm{UC}$, in distribution. Let $\boldsymbol{\tau}^{\varepsilon}=\inf \left\{\mathbf{x} \geqslant 0: \mathbf{B}_{\varepsilon}(\mathbf{x}) \geqslant \mathfrak{g}_{\varepsilon}(\mathbf{x})\right\}$ and $\boldsymbol{\tau}=\inf \{\mathbf{x} \geqslant 0: \mathbf{B}(\mathbf{x}) \geqslant \mathfrak{g}(\mathbf{x})\}$ be the first hitting times of $\operatorname{epi}\left(\mathfrak{g}_{\varepsilon}\right)$ and $\operatorname{epi}(\mathfrak{g})$, respectively. Then, $\boldsymbol{\tau}^{\varepsilon} \rightarrow \boldsymbol{\tau}$ in distribution. Furthermore, the convergence is uniform over $\mathfrak{g}$ in sets of bounded Hölder $\beta$-norm, $\beta \in\left(0, \frac{1}{2}\right)$.

Proof. If there exists a subsequence $\boldsymbol{\tau}^{\varepsilon_{n}} \rightarrow \mathbf{x}$, then

$$
0 \leqslant \limsup \mathbf{B}_{\varepsilon_{n}}\left(\boldsymbol{\tau}^{\varepsilon_{n}}\right)-\mathfrak{g}_{\varepsilon_{n}}\left(\operatorname{ftau}^{\varepsilon_{n}}\right) \leqslant \mathbf{B}(\mathbf{x})-\mathfrak{g}(\mathbf{x}),
$$

because they are converging in UC, so $\mathbf{x} \geqslant \boldsymbol{\tau}$, and thus $\liminf \boldsymbol{\tau}^{\varepsilon} \geqslant \boldsymbol{\tau}$. For the other direction, let $f_{\delta}(0)=0, f_{\delta}(\mathbf{x})=\delta$ for $\mathbf{x} \geqslant \delta$, and linear in between. Let

$$
\overline{\boldsymbol{\tau}}_{\delta}=\inf \left\{\mathbf{x} \geqslant 0: \mathbf{B}(\mathbf{x}) \geqslant \mathfrak{g}(\mathbf{x})+f_{\delta}(\mathbf{x})\right\} .
$$

By the Cameron-Martin formula, $\overline{\boldsymbol{\tau}}_{\delta} \searrow \boldsymbol{\tau}$ in distribution:

$$
\mathbb{P}\left(\overline{\boldsymbol{\tau}}_{\delta} \in I\right)=\mathbb{E}\left[\exp \left\{B(\delta)-B(0)-\frac{1}{2} \delta\right\} \mathbf{1}_{\boldsymbol{\tau} \in I}\right] \rightarrow \mathbb{P}(\boldsymbol{\tau} \in I),
$$

since the integrands are uniformly integrable. Since $\mathbf{B}_{\varepsilon}-\mathfrak{g}_{\varepsilon} \rightarrow \mathbf{B}-\mathfrak{g}$ in UC, there are $\mathbf{x}_{\varepsilon} \rightarrow \overline{\boldsymbol{\tau}}_{\delta}$ with

$$
\liminf \left(\mathbf{B}_{\varepsilon}\left(\mathbf{x}_{\varepsilon}\right)-\mathfrak{g}_{\varepsilon}\left(\mathbf{x}_{\varepsilon}\right)\right)>0 .
$$


So, $\boldsymbol{\tau}^{\varepsilon}<\overline{\boldsymbol{\tau}}_{\delta}+\delta$ for $\varepsilon$ sufficiently small. This proves that $\limsup \boldsymbol{\tau}^{\varepsilon} \leqslant \boldsymbol{\tau}$ in distribution.

To prove the uniformity, for any $\delta>0$, and restricting to $[0, M]$, we have

$$
\operatorname{hypo}\left(\mathbf{B}_{\varepsilon}-\mathfrak{g}_{\varepsilon}\right) \subset B_{\delta}(\operatorname{hypo}(\mathbf{B}-\mathfrak{g}))
$$

for sufficiently small $\varepsilon>0$. There is a $\gamma>0$ depending only on the Hölder $\beta$-norm of $\mathbf{B}-\mathfrak{g}$ and going to zero, with $\delta$ such that

$$
B_{\delta}(\operatorname{hypo}(\mathbf{B}-\mathfrak{g})) \subset \operatorname{hypo}(\mathbf{B}-\mathfrak{g}+\gamma)
$$

Hence, $\boldsymbol{\tau}^{\varepsilon} \geqslant \boldsymbol{\tau}_{z+\gamma}$ in distribution, where the subscript $z+\gamma$ indicates that the hitting time $\boldsymbol{\tau}_{z+\gamma}$ is for the Brownian starting with $\mathbf{B}(0)=z+\gamma$. In the other direction, for any $\delta>0$ there exists $\gamma>0$ depending only on the Hölder $\beta$-norm of $\mathbf{B}-\mathfrak{g}$ such that $\mathbf{B}(\mathbf{x})-\mathfrak{g}(\mathbf{x})>\delta$ for any $\left|\mathbf{x}-\boldsymbol{\tau}_{z-\gamma}\right|<\delta$, and there exist $\left|\mathbf{x}_{\delta}-\boldsymbol{\tau}_{z-\gamma}\right|<\delta$ for which

$$
\left(\mathbf{B}_{\varepsilon}-\mathfrak{g}_{\varepsilon}\right)\left(\mathbf{x}_{\delta}\right) \geqslant(\mathbf{B}-\mathfrak{g})\left(\boldsymbol{\tau}_{z-\gamma}\right)-\delta
$$

Hence, $\boldsymbol{\tau}^{\varepsilon} \leqslant \mathbf{x}_{\delta}<\boldsymbol{\tau}_{z-\gamma}+\delta$. Now, let $(\overline{\mathbf{B}}, \underline{\mathbf{B}})$ be a pair of coalescing Brownian motions starting at $(z+\gamma, z-\gamma)$ defined by letting $\overline{\mathbf{B}}(\mathbf{y})=2 z-\underline{\mathbf{B}}(\mathbf{y})$ until the first time $\boldsymbol{\sigma}_{z}$ they meet, and $\overline{\mathbf{B}}(\mathbf{y})=\underline{\mathbf{B}}(\mathbf{y})$ for $\mathbf{y}>\boldsymbol{\sigma}_{z}$. We have

$$
\mathbb{P}\left(\boldsymbol{\tau}_{z+\gamma} \leqslant T\right)-\mathbb{P}\left(\boldsymbol{\tau}_{z-\gamma} \leqslant T\right)=\mathbb{P}(\overline{\mathbf{B}} \text { hit but } \mathbf{B} \text { did not }) \leqslant \mathbb{P}\left(\boldsymbol{\sigma}_{z}>\boldsymbol{\tau}_{z+\gamma}\right)
$$

Recall that $z<\mathfrak{g}(\mathbf{x})$. We also have

$$
\mathfrak{g}(\mathbf{x}+\mathbf{y}) \geqslant \mathfrak{g}(\mathbf{x})-C \mathbf{y}^{\beta}
$$

for $0 \leqslant \mathbf{y} \leqslant T$ by the uniform Hölder bound, so if we let $\boldsymbol{\nu}_{z+\gamma}$ be the hitting time of $\mathfrak{g}(\mathbf{x})-C \mathbf{y}^{\beta}$ by $\overline{\mathbf{B}}$, then we get $\boldsymbol{\tau}_{z+\gamma} \geqslant \boldsymbol{\nu}_{z+\gamma}$. Hence,

$$
\mathbb{P}\left(\boldsymbol{\tau}_{z+\gamma} \leqslant T\right)-\mathbb{P}\left(\boldsymbol{\tau}_{z-\gamma} \leqslant T\right) \leqslant \mathbb{P}\left(\boldsymbol{\sigma}_{z}>\boldsymbol{\nu}_{z+\gamma}\right) \rightarrow 0
$$

as $\gamma \rightarrow 0$, with a rate depending only on $C, \gamma$ and $\beta$.

\subsection{Approximation setup}

For any $\mathfrak{h}_{0} \in \mathrm{UC}$, we can find initial data $X_{0}^{\varepsilon}$ so that (3.4) holds in the LC topology. This is easy to see, because any $\mathfrak{h}_{0} \in \mathrm{UC}$ is the limit of functions which are finite at finitely many points, and $-\infty$ otherwise. In turn, such functions can be approximated by initial data $X_{0}^{\varepsilon}$, where the particles are densely packed in blocks. Note that there is a mild 
abuse here, as the left-hand side of (3.4) is a function on $\varepsilon \mathbb{Z}$. We can always extend it to $\mathbb{R}$ in a simple way, say taking it piecewise constant on $(\varepsilon n, \varepsilon(n+1))$, and choosing the endpoints so that it is lower semi-continuous. Similarly, (3.1) will be taken to be a piecewise constant UC function.

Our goal is to take such a sequence of initial data $X_{0}^{\varepsilon}$ and compute

$$
\mathbb{P}_{\mathfrak{h}_{0}}\left(\mathfrak{h}\left(\mathbf{t}, \mathbf{x}_{i}\right) \leqslant \mathbf{a}_{i} \text { for } i=1, \ldots, m\right),
$$

which, from (2.1), (3.1), (3.3) and (3.5), is the limit as $\varepsilon \rightarrow 0$ of

$$
\mathbb{P}_{X_{0}}\left(X_{2 \varepsilon^{-3 / 2} \mathbf{t}}\left(\frac{1}{2} \varepsilon^{-3 / 2} \mathbf{t}-\varepsilon^{-1} \mathbf{x}_{i}-\frac{1}{2} \varepsilon^{-1 / 2} \mathbf{a}_{i}+1\right)>2 \varepsilon^{-1} \mathbf{x}_{i}-2 \text { for } i=1, \ldots, m\right) .
$$

We therefore want to consider Theorem 2.6 with

$$
t=2 \varepsilon^{-3 / 2} \mathbf{t}, \quad n_{i}=\frac{1}{2} \varepsilon^{-3 / 2} \mathbf{t}-\varepsilon^{-1} \mathbf{x}_{i}-\frac{1}{2} \varepsilon^{-1 / 2} \mathbf{a}_{i}+1 \quad \text { and } \quad a_{i}=2 \varepsilon^{-1} \mathbf{x}_{i}-2,
$$

where we will always assume that $\varepsilon$ is small enough so that $n_{i}>0$ for each $i$.

The formula (2.31) for the TASEP kernel requires initial data which is right-finite. While one can build a formula which holds without this restriction, it is not nice for passing to limits. But there is no loss of generality in considering right-finite data because of the next lemma, which says that we can safely cut off our data far to the right. It also tells us how fast information is transmitted in the fixed point (see Theorem 4.6).

Definition 3.3. (Cutoff data) For each integer $L$, the cutoff data is

$$
X_{0}^{\varepsilon, L}(n)= \begin{cases}X_{0}^{\varepsilon}(n), & \text { if } n>-\left\lfloor\varepsilon^{-1} L^{\prime}\right\rfloor, \\ \infty, & \text { if } n \leqslant-\left\lfloor\varepsilon^{-1} L^{\prime}\right\rfloor,\end{cases}
$$

where $L^{\prime} \sim \frac{1}{2} L$ is chosen so that $\varepsilon X_{0}^{\varepsilon}\left(-\left\lfloor\varepsilon^{-1} L^{\prime}\right\rfloor\right)=L$. This corresponds to replacing $\mathfrak{h}_{0}^{\varepsilon}(\mathbf{x})$ by $\mathfrak{h}_{0}^{\varepsilon, L}(\mathbf{x})$, with a straight line with slope $-2 \varepsilon^{-1 / 2}$ to the right of $L$. This is the UC cutoff at $L$. The LC cutoff of $\mathfrak{g}$ at $L$ is just minus the UC cutoff of $\mathfrak{g}$.

The following will be proved in Appendix C.1.

Lemma 3.4. (Finite propagation speed) Suppose $X_{0}^{\varepsilon}$ satisfies (3.4), with $\mathfrak{h}_{0} \in \mathrm{UC}$. There are $\varepsilon_{0}>0$ and $C<\infty, \delta>0$ independent of $\varepsilon \in\left(0, \varepsilon_{0}\right)$ such that the difference of $(3.7)$ computed with initial data $X_{0}^{\varepsilon}$ and with initial data $X_{0}^{\varepsilon, L}$ is bounded by $C e^{-(2 / 3-\delta) L^{3}}$.

\subsection{Limiting operators}

The limits are stated in terms of an (almost) group of operators

$$
\mathbf{S}_{\mathbf{t}, \mathbf{x}}=\exp \left\{\mathbf{x} \partial^{2}+\frac{1}{3} \mathbf{t} \partial^{3}\right\}, \quad \mathbf{x}, \mathbf{t} \in \mathbb{R}^{2} \backslash\{\mathbf{x}<0, \mathbf{t}=0\},
$$


satisfying $\mathbf{S}_{\mathbf{s}, \mathbf{x}} \mathbf{S}_{\mathbf{t}, \mathbf{y}}=\mathbf{S}_{\mathbf{s}+\mathbf{t}, \mathbf{x}+\mathbf{y}}$, as long as all subscripts avoid $\{\mathbf{x}<0, \mathbf{t}=0\}$. We can think of them as unbounded operators with domain $\mathscr{C}_{0}^{\infty}(\mathbb{R})$. It is somewhat surprising that they even make sense for $\mathbf{x}<0, \mathbf{t} \neq 0$, but it is just an elementary consequence of the following explicit kernel and basic properties of the Airy function $\left({ }^{17}\right)$

$$
\operatorname{Ai}(z)=\frac{1}{2 \pi i} \int_{<} d w e^{w^{3} / 3-z w}
$$

The $\mathbf{S}_{\mathbf{t}, \mathbf{x}}$ act by convolution:

$$
\mathbf{S}_{\mathbf{t}, \mathbf{x}} f(z)=\int_{-\infty}^{\infty} d y \mathbf{S}_{\mathbf{t}, \mathbf{x}}(z, y) f(y)=\int_{-\infty}^{\infty} d y \mathbf{S}_{\mathbf{t}, \mathbf{x}}(z-y) f(y)
$$

where, for $\mathbf{t}>0$,

$$
\mathbf{S}_{\mathbf{t}, \mathbf{x}}(z)=\frac{1}{2 \pi i} \int_{\langle} d w e^{\mathbf{t} w^{3} / 3+\mathbf{x} w^{2}+z w}=\mathbf{t}^{-1 / 3} e^{2 \mathbf{x}^{3} / 3 \mathbf{t}^{2}-z \mathbf{x} / \mathbf{t}} \operatorname{Ai}\left(-\mathbf{t}^{-1 / 3} z+\mathbf{t}^{-4 / 3} \mathbf{x}^{2}\right),
$$

and $\mathbf{S}_{-\mathbf{t}, \mathbf{x}}=\left(\mathbf{S}_{\mathbf{t}, \mathbf{x}}\right)^{*}$, or $\mathbf{S}_{-\mathbf{t}, \mathbf{x}}(z, y)=\mathbf{S}_{-\mathbf{t}, \mathbf{x}}(z-y)=\mathbf{S}_{\mathbf{t}, \mathbf{x}}(y-z)$. From this, we get directly the identity $\left(\mathbf{S}_{\mathbf{t}, \mathbf{x}}\right)^{*} \mathbf{S}_{\mathbf{t},-\mathbf{x}}=\mathbf{I}$, which we will use often without reference.

In addition to $\mathbf{S}_{\mathbf{t}, \mathbf{x}}$, we need to introduce the limiting version of $\overline{\mathcal{S}}_{t, n}^{\text {epi }\left(X_{0}\right)}$. It will actually be more convenient for us to introduce the hypograph variant of this operator first, since it is the one that will show up more often in our formulas: for $\mathfrak{h} \in U C$, we define

$$
\mathbf{S}_{\mathbf{t}, \mathbf{x}}^{\text {hypo(h) }}(v, u)=\mathbb{E}_{\mathbf{B}(0)=v}\left[\mathbf{S}_{\mathbf{t}, \mathbf{x}-\boldsymbol{\tau}}(\mathbf{B}(\boldsymbol{\tau}), u) \mathbf{1}_{\boldsymbol{\tau}<\infty}\right],
$$

where $\mathbf{B}(x)$ is a Brownian motion with diffusion coefficient 2, and $\boldsymbol{\tau}$ is the hitting time of the hypograph of $\mathfrak{h} .\left({ }^{18}\right),\left({ }^{19}\right)$ Note that, trivially, $\mathbf{S}_{\mathbf{t}, \mathbf{x}}^{\text {hypo(h) }}(v, u)=\mathbf{S}_{\mathbf{t}, \mathbf{x}}(v, u)$ for $v \leqslant \mathfrak{h}(0)$. The fact that the expectation in (3.11) is finite will be proved in Appendix A. We have that $\mathbf{S}_{\mathbf{t}, \mathbf{x}}^{\text {hypo(h) }}$ really depends only on the values of $\mathfrak{h}$ on $[0, \infty)$, and we will sometimes evaluate it at functions defined only there.

One way to think of $\mathbf{S}_{\mathbf{t}, \mathbf{x}}^{\text {hypo(h) }}(v, u)$ is as a sort of asymptotic transformed transition density for the Brownian motion $\mathbf{B}$ to go from $v$ to $u$ hitting the hypograph of $\mathfrak{h}$. To see

$\left({ }^{17}\right)<$ is the positively oriented contour going from $e^{-i \pi / 3} \infty$ to $e^{i \pi / 3} \infty$ through zero.

$\left({ }^{18}\right)$ It is important that we use $\mathbf{B}(\boldsymbol{\tau})$ in (3.11) and not $\mathfrak{h}(\boldsymbol{\tau})$ which, for discontinuous initial data, could be strictly larger.

$\left({ }^{19}\right)$ We have that $\mathbf{S}_{\mathbf{t}, \mathbf{x}-\mathbf{y}}(\mathbf{B}(\mathbf{y}), u)$ is a martingale in $\mathbf{y} \geqslant 0$. However, it is not uniformly integrable and one cannot apply the optional stopping theorem to conclude that $\mathbb{E}_{\mathbf{B}(0)=v}\left[\mathbf{S}_{\mathbf{t}, \mathbf{x}-\boldsymbol{\tau}}(\mathbf{B}(\boldsymbol{\tau}), u) \mathbf{1}_{\boldsymbol{\tau}<\infty}\right]=$ $\mathbf{S}_{\mathbf{t}, \mathbf{x}}(v, u)$. For example, if $\mathfrak{h} \equiv 0$, one gets instead

$$
\mathbb{E}_{\mathbf{B}(0)=v}\left[\mathbf{S}_{\mathbf{t}, \mathbf{x}-\boldsymbol{\tau}}(\mathbf{B}(\boldsymbol{\tau}), u) \mathbf{1}_{\boldsymbol{\tau}<\infty}\right]=\mathbf{S}_{\mathbf{t}, \mathbf{x}}(-v, u)
$$

for $v>0$ (see [51, Proposition 3.6]). 
what we mean, write

$$
\begin{gathered}
\mathbf{S}_{\mathbf{t}, \mathbf{x}}^{\text {hypo(h) }}=\lim _{\mathbf{T} \rightarrow \infty} \mathbf{S}_{[0, \mathbf{T}]}^{\text {hypo(h) }} \mathbf{S}_{\mathbf{t}, \mathbf{x}-\mathbf{T}} \\
\text { with } \mathbf{S}_{[0, \mathbf{T}]}^{\text {hypo }(\mathfrak{h})}(v, u)=\mathbb{E}_{\mathbf{B}(0)=v}\left[\mathbf{S}_{0, \mathbf{T}-\boldsymbol{\tau}}(\mathbf{B}(\boldsymbol{\tau}), u) \mathbf{1}_{\boldsymbol{\tau} \leqslant \mathbf{T}}\right],
\end{gathered}
$$

and note that $\mathbf{S}_{[0, \mathbf{T}]}^{\text {hypo(h) }}(v, u)$ is nothing but the transition density for $\mathbf{B}$ to go from $v$ at time zero to $u$ at time $\mathbf{T}$ hitting hypo $(\mathfrak{h})$ in $[0, \mathbf{T}]$.

The epi version of the operator is defined similarly: for $\mathfrak{g} \in \mathrm{LC}$,

$$
\mathbf{S}_{\mathbf{t}, \mathbf{x}}^{\mathrm{epi}(\mathfrak{g})}(v, u)=\mathbb{E}_{\mathbf{B}(0)=v}\left[\mathbf{S}_{\mathbf{t}, \mathbf{x}-\boldsymbol{\tau}}(\mathbf{B}(\boldsymbol{\tau}), u) \mathbf{1}_{\boldsymbol{\tau}<\infty}\right],
$$

where $\boldsymbol{\tau}$ is now defined as the hitting time of the epigraph of $\mathfrak{g}$ (the meaning of $\boldsymbol{\tau}$ will always be clear from the context); now we have $\mathbf{S}_{\mathbf{t}, \mathbf{x}}^{\mathrm{epi}(\mathfrak{h})}(v, u)=\mathbf{S}_{\mathbf{t}, \mathbf{x}}(v, u)$ for $v \geqslant \mathfrak{g}(0)$. As a consequence of (3.12), one can see that the epi and hypo operators are related through

$$
\mathbf{S}_{-\mathbf{t}, \mathbf{x}}^{\mathrm{epi}(\mathfrak{g})}(v, u)=\mathbf{S}_{\mathbf{t}, \mathbf{x}}^{\mathrm{hypo}(-\mathfrak{g})}(-v,-u) .
$$

LEMma 3.5. Under the scaling (3.8) (dropping the $i$ subscripts) and assuming that (3.4) holds in LC, if we set $z=2 \varepsilon^{-1} \mathbf{x}+\varepsilon^{-1 / 2}(u+\mathbf{a})-2$ and $y^{\prime}=\varepsilon^{-1 / 2} v$, then we have for $\mathbf{t}>0$ as $\varepsilon \searrow 0$,

$$
\begin{aligned}
& \mathbf{S}_{-\mathbf{t}, \mathbf{x}}^{\varepsilon}(v, u):=\varepsilon^{-1 / 2} \mathcal{S}_{-t,-n}\left(y^{\prime}, z\right) \rightarrow \mathbf{S}_{-\mathbf{t}, \mathbf{x}}(v, u), \\
& \overline{\mathbf{S}}_{-\mathbf{t},-\mathbf{x}}^{\varepsilon}(v, u):=\varepsilon^{-1 / 2} \overline{\mathcal{S}}_{-t, n}\left(y^{\prime}, z\right) \rightarrow \mathbf{S}_{-\mathbf{t},-\mathbf{x}}(v, u), \\
& \overline{\mathbf{S}}_{-\mathbf{t},-\mathbf{x}}^{\left.\varepsilon,\left(\mathfrak{h}_{0}^{\varepsilon}\right)^{-}\right)}(v, u):=\varepsilon^{-1 / 2} \overline{\mathcal{S}}_{-t, n}^{\mathrm{epi}\left(X_{0}\right)}\left(y^{\prime}, z\right) \rightarrow \mathbf{S}_{-\mathbf{t},-\mathbf{x}}^{\mathrm{epi}\left(-\mathfrak{h}_{0}^{-}\right)}(v, u)
\end{aligned}
$$

pointwise, where $\mathfrak{h}^{-}(x)=\mathfrak{h}(-x)$ for $x \geqslant 0$. Here, $\mathcal{S}_{-t,-n}, \overline{\mathcal{S}}_{-t, n}$ are defined in (2.27) and (2.28).

Note that the kernels on the left-hand side also depend on a, but we will not write the dependence explicitly.

The pointwise convergence does not actually suffice for our purposes; it will be suitably upgraded in Appendix B. The asymptotics in Lemma 3.5 is elementary, and not really a steepest descent. Where steepest descent is needed is in Appendix B, to study the asymptotics in $\mathbf{x}, v$ amd $u_{i}$ of the approximating functions on the left-hand sides of (3.15)-(3.17), in order to bound the kernels in trace norm (see $\S \mathrm{B})$.

Proof. First, we give a heuristic proof using operators, which helps one understand where the third derivative comes from. As $Q^{-1}=I+2 \nabla^{+}$, with $\nabla^{+} f(x)=f(x+1)-f(x)$, and dropping lower-order terms, the left-hand side of (3.15) is

$$
e^{-\varepsilon^{-3 / 2} \mathbf{t} \nabla^{-}} Q^{-\varepsilon^{-3 / 2} \mathbf{t} / 2}=e^{\varepsilon^{-3 / 2} \mathbf{t}\left[-\nabla^{-}+(1 / 2) \log \left(I+2 \nabla^{+}\right)\right]} .
$$


The scaled lattice is $\varepsilon^{1 / 2} \mathbb{Z}$, so $\nabla^{ \pm} \sim \varepsilon^{1 / 2}$, and therefore

$$
-\nabla^{-}+\frac{1}{2} \log \left(I+2 \nabla^{+}\right)=-\nabla^{-}+\nabla^{+}-\left(\nabla^{+}\right)^{2}+\frac{4}{3}\left(\nabla^{+}\right)^{3}+\mathcal{O}\left(\varepsilon^{2}\right) .
$$

Now,

$$
\begin{aligned}
\left(-\nabla^{-}+\right. & \left.\nabla^{+}-\left(\nabla^{+}\right)^{2}+\frac{4}{3}\left(\nabla^{+}\right)^{3}\right) f(x) \\
= & \frac{4}{3}\left(f\left(x+3 \varepsilon^{1 / 2}\right)-3 f\left(x+2 \varepsilon^{1 / 2}\right)+3 f\left(x+\varepsilon^{1 / 2}\right)-f(x)\right) \\
& \quad-\frac{1}{2}\left(f\left(x+2 \varepsilon^{1 / 2}\right)-3 f\left(x+\varepsilon^{1 / 2}\right)+3 f(x)-f\left(x-\varepsilon^{1 / 2}\right)\right) \\
\sim & \frac{1}{3} \varepsilon^{3 / 2} \partial^{3} f(x) .
\end{aligned}
$$

We also have $Q^{\varepsilon^{-1} x} \sim e^{x \partial^{2}}$ under our scaling, by the central limit theorem. This explains how (3.9) arises.

Now we switch to the rigorous proof, which uses the contour integral representations. Note that $\mathbf{S}_{-\mathbf{t}, \mathbf{x}}^{\varepsilon}(v, u)$ and $\overline{\mathbf{S}}_{-\mathbf{t}, \mathbf{x}}^{\varepsilon}(v, u)$ only depend on $v-u$. Writing

$$
\mathbf{S}_{-\mathbf{t}, \mathbf{x}}^{\varepsilon}(u)=\mathbf{S}_{-\mathbf{t}, \mathbf{x}}^{\varepsilon}(u, 0) \quad \text { and } \quad \overline{\mathbf{S}}_{-\mathbf{t}, \mathbf{x}}^{\varepsilon}(u)=\overline{\mathbf{S}}_{-\mathbf{t}, \mathbf{x}}^{\varepsilon}(u, 0)
$$

changing variables $w \mapsto \frac{1}{2}\left(1-\varepsilon^{1 / 2} \widetilde{w}\right)$ in $(2.27)$ and (2.28), and using the scaling (3.8), we have

$$
\begin{aligned}
\mathbf{S}_{-\mathbf{t}, \mathbf{x}}^{\varepsilon}(u) & =\frac{1}{2 \pi i} \oint_{C_{\varepsilon}} d \widetilde{w} e^{\varepsilon^{-3 / 2} \mathbf{t} F\left(\varepsilon^{1 / 2} \widetilde{w}, \varepsilon^{1 / 2} \mathbf{x}_{\varepsilon} / \mathbf{t}, \varepsilon u_{\varepsilon} / \mathbf{t}\right)}, \\
\overline{\mathbf{S}}_{-\mathbf{t}, \mathbf{x}}^{\varepsilon}(u) & =\frac{1}{2 \pi i} \oint_{C_{\varepsilon}} d \widetilde{w} e^{\varepsilon^{-3 / 2} \mathbf{t} F\left(\varepsilon^{1 / 2} \widetilde{w}, \varepsilon^{1 / 2} \overline{\mathbf{x}}_{\varepsilon} / \mathbf{t}, \varepsilon \bar{u}_{\varepsilon} / \mathbf{t}\right)} \\
F(w, x, u) & =\operatorname{arctanh} w-w-x \log \left(1-w^{2}\right)-u \operatorname{arctanh} w
\end{aligned}
$$

where

$$
\begin{array}{ll}
\mathbf{x}_{\varepsilon}=\mathbf{x}-\frac{1}{2} \varepsilon^{1 / 2}(u-\mathbf{a})-\frac{1}{2} \varepsilon, & u_{\varepsilon}=u-\varepsilon^{1 / 2} \\
\overline{\mathbf{x}}_{\varepsilon}=\mathbf{x}+\frac{1}{2} \varepsilon^{1 / 2}(u-\mathbf{a})+\frac{3}{2} \varepsilon, & \bar{u}_{\varepsilon}=u+\varepsilon^{1 / 2}
\end{array}
$$

We have that $C_{\varepsilon}$ is a circle of radius $\varepsilon^{-1 / 2}$ centered at $\varepsilon^{-1 / 2}$, and

$$
\operatorname{arctanh} w=\frac{1}{2}[\log (1+w)-\log (1-w)]
$$

It is striking how similar the formulas are in this representation and scaling, even if $\overline{\mathbf{S}}_{-\mathbf{t}, \mathbf{x}}^{\varepsilon}(u)$ comes from an analytic extension of $\mathbf{S}_{-\mathbf{t}, \mathbf{x}}^{\varepsilon}(u)$. Note that

$$
\partial_{w} F(w, x, u)=\left(w-w_{+}\right)\left(w-w_{-}\right)\left(1-w^{2}\right)^{-1}
$$


with

$$
w_{ \pm}=w_{ \pm}(x, u):=-x \pm \sqrt{x^{2}+u}
$$

so, in particular,

$$
\partial_{w} F\left(\varepsilon^{1 / 2} \widetilde{w}, \frac{\varepsilon^{1 / 2} \mathbf{x}_{\varepsilon}}{\mathbf{t}}, \frac{\varepsilon u_{\varepsilon}}{\mathbf{t}}\right)=\varepsilon^{3 / 2}\left(\widetilde{w}-w_{+}^{\varepsilon}\right)\left(\widetilde{w}-w_{-}^{\varepsilon}\right)\left(1-\varepsilon \widetilde{w}^{2}\right)^{-1},
$$

with

$$
w_{ \pm}^{\varepsilon}=w_{ \pm}\left(\frac{\mathbf{x}_{\varepsilon}}{\mathbf{t}}, \frac{u_{\varepsilon}}{\mathbf{t}}\right) .
$$

Keeping in mind that $\mathbf{S}_{-\mathbf{t}, \mathbf{x}}=\left(\mathbf{S}_{\mathbf{t}, \mathbf{x}}\right)^{*}$, from (3.10) we see then that, as $\varepsilon \searrow 0$, the exponents in (3.18) and (3.19) converge to the correct exponents in (3.16) and (3.17). Deform $C_{\varepsilon}$ to the contour $\left\langle_{\varepsilon} \cup C_{\varepsilon}^{\pi / 3}\right.$, where $\left\langle_{\varepsilon}\right.$ is the part of the Airy contour $\langle$ (see footnote 17) within the ball of radius $\varepsilon^{-1 / 2}$ centered at $\varepsilon^{-1 / 2}$, and $C_{\varepsilon}^{\pi / 3}$ is the part of $C_{\varepsilon}$ to the right of $\langle$. As $\varepsilon \searrow 0$, we have $\left\langle_{\varepsilon} \rightarrow\langle\right.$, and it is easy to see that the integral over the part of $\langle$ which is not in $\left\langle_{\varepsilon}\right.$ goes to zero. So, it only remains to show that the integral over $C_{\varepsilon}^{\pi / 3}$ converges to zero. To see this, note that the real part of the exponent of the integral over $C_{\varepsilon}$ in (3.18), parameterized as $\widetilde{w}=\varepsilon^{-1 / 2}\left(1+e^{i \theta}\right)$, is given by

$$
\varepsilon^{-3 / 2} \mathbf{t}\left[-1-\cos (\theta)+\left(\frac{1}{4}+\mathcal{O}\left(\varepsilon^{1 / 2}\right)\right) \log (5+4 \cos (\theta))\right] .
$$

Using $\log (1+x) \leqslant x$ for $x \geqslant 0$, this is bounded by $\frac{1}{2} \varepsilon^{-3 / 2} \mathbf{t}[-1-\cos (\theta)-\log (2)]$ for sufficiently small $\varepsilon$. The $\widetilde{w} \in C_{\varepsilon}^{\pi / 3}$ correspond to $|\theta| \leqslant \frac{1}{3} \pi$, so the exponent there is less than $-\varepsilon^{-3 / 2} \kappa \mathbf{t}$ for some $\kappa>0$. Hence, this part of the integral vanishes.

Now, define the scaled walk

$$
\mathbf{B}_{\varepsilon}(\mathbf{x})=\varepsilon^{1 / 2}\left(B_{\varepsilon^{-1} \mathbf{x}}+2 \varepsilon^{-1} \mathbf{x}-1\right)
$$

for $\mathbf{x} \in \varepsilon \mathbb{Z}_{\geqslant 0}$, interpolated linearly in between, and let $\boldsymbol{\tau}^{\varepsilon}$ be the hitting time by $\mathbf{B}_{\varepsilon}$ of epi $\left(-\left(\mathfrak{h}_{0}^{\varepsilon}\right)^{-}\right)$. By Donsker's invariance principle [7], $\mathbf{B}_{\varepsilon}(\mathbf{x})$ converges locally uniformly in distribution to a Brownian motion $\mathbf{B}(\mathbf{x})$ with diffusion coefficient 2, and therefore (using (3.4) and Proposition 3.2) the hitting time $\boldsymbol{\tau}^{\varepsilon}$ converges to $\boldsymbol{\tau}$ as well.

We will next compute the limit of (3.7), using (2.30) under the scaling (3.8). To this end, we change variables in the kernel as in Lemma 3.5, so that, for

$$
z_{i}=2 \varepsilon^{-1} \mathbf{x}_{i}+\varepsilon^{-1 / 2}\left(u_{i}+\mathbf{a}_{i}\right)-2,
$$

we need to compute the limit of

$$
\varepsilon^{-1 / 2}\left(\bar{\chi}_{2 \varepsilon^{-1} \mathbf{x}-2} K_{t} \bar{\chi}_{2 \varepsilon^{-1} \mathbf{x}-2}\right)\left(z_{i}, z_{j}\right) .
$$


Note that the change of variables turns $\bar{\chi}_{2 \varepsilon^{-1} \mathbf{x}-2}(z)$ into $\bar{\chi}_{-\mathbf{a}}(u)$. We have $n_{i}<n_{j}$ for small $\varepsilon$ if and only if $\mathbf{x}_{j}<\mathbf{x}_{i}$, and in this case we have, under our scaling,

$$
\varepsilon^{-1 / 2} Q^{n_{j}-n_{i}}\left(z_{i}, z_{j}\right) \rightarrow e^{\left(\mathbf{x}_{i}-\mathbf{x}_{j}\right) \partial^{2}}\left(u_{i}, u_{j}\right),
$$

as $\varepsilon \searrow 0$. The rescaled second term in $(2.31), \varepsilon^{-1 / 2}\left(\mathcal{S}_{-t,-n_{i}}\right)^{*} \overline{\mathcal{S}}_{-t, n_{j}}^{\operatorname{epi}\left(X_{0}\right)}\left(z_{i}, z_{j}\right)$, can be written as $\left(\mathbf{S}_{-\mathbf{t}, \mathbf{x}_{i}}^{\varepsilon}\right)^{*} \mathbf{S}_{-\mathbf{t},-\mathbf{x}_{j}}^{\left.\left.\varepsilon, \mathfrak{h}_{0}^{\varepsilon}\right)^{-}\right)}\left(u_{i}, u_{j}\right)$, and we can read off from Lemma 3.5 that this can be expected to converge to $\left(\mathbf{S}_{-\mathbf{t}, \mathbf{x}_{i}}\right)^{*} \mathbf{S}_{-\mathbf{t},-\mathbf{x}_{j}}^{\mathrm{epi}\left(-\mathfrak{h}_{0}^{-}\right)}\left(u_{i}, u_{j}\right)$. The limiting kernel

$$
\mathbf{K}_{\lim }:=e^{\left(\mathbf{x}_{i}-\mathbf{x}_{j}\right) \partial^{2}} \mathbf{1}_{\mathbf{x}_{j}<\mathbf{x}_{i}}+\left(\mathbf{S}_{-\mathbf{t}, \mathbf{x}_{i}}\right)^{*} \mathbf{S}_{-\mathbf{t},-\mathbf{x}_{j}}^{\mathrm{epi}\left(-\mathfrak{h}_{0}^{-}\right)}
$$

would be surrounded by projections $\bar{\chi}_{-\mathbf{a}}$. For aesthetic reasons, it is nicer to have projections $\chi_{\mathbf{a}}$, so we change variables $u_{i} \mapsto-u_{i}$ and replace the Fredholm determinant of the kernel by that of its adjoint to get

$$
\operatorname{det}\left(\mathbf{I}-\chi_{\mathbf{a}} \mathbf{K}_{\mathbf{t}, \text { ext }}^{\text {hypo }\left(\mathfrak{h}_{0}\right)} \chi_{\mathbf{a}}\right),
$$

with

$$
\mathbf{K}_{\mathbf{t}, \text { ext }}^{\text {hypo }\left(\mathfrak{h}_{0}\right)}\left(u_{i}, u_{j}\right)=\mathbf{K}_{\lim }\left(\mathbf{x}_{j},-u_{j} ; \mathbf{x}_{i},-u_{i}\right) .
$$

The choice of superscript hypo $\left(\mathfrak{h}_{0}\right)$ in the resulting kernel comes from (3.14), which together with $\mathbf{S}_{-\mathbf{t}, \mathbf{x}}(-u, v)=\left(\mathbf{S}_{\mathbf{t}, \mathbf{x}}\right)^{*}(-v, u)$ yield

Proposition 3.6. (One-sided fixed point formula) Let $\mathfrak{h}_{0} \in \mathrm{UC}$ with $\mathfrak{h}_{0}(\mathbf{x})=-\infty$ for $\mathbf{x}>0$. Assume that we start TASEP with right-finite initial data $X_{0}$ such that the rescaled height function $\mathfrak{h}^{\varepsilon}(\mathbf{t}, \mathbf{x})$ given by (3.1) satisfies $\mathfrak{h}_{0}^{\varepsilon}(\mathbf{x}):=\mathfrak{h}^{\varepsilon}(0, \mathbf{x}) \rightarrow \mathfrak{h}_{0}(\mathbf{x})$ in distribution in $\mathrm{UC}$ as $\varepsilon \rightarrow 0$. Then, for any distinct $\mathbf{x}_{1}, \ldots, \mathbf{x}_{m} \in \mathbb{R}$ and any $\mathbf{a}_{1}, \ldots, \mathbf{a}_{m} \in \mathbb{R}$, we have

$$
\lim _{\varepsilon \rightarrow 0} \mathbb{P}_{\mathfrak{h}_{0}^{\varepsilon}}\left(\mathfrak{h}^{\varepsilon}\left(\mathbf{t}, \mathbf{x}_{1}\right) \leqslant \mathbf{a}_{1}, \ldots, \mathfrak{h}^{\varepsilon}\left(\mathbf{t}, \mathbf{x}_{m}\right) \leqslant \mathbf{a}_{m}\right)=\operatorname{det}\left(\mathbf{I}-\chi_{\mathbf{a}} \mathbf{K}_{\mathbf{t}, \text { ext }}^{\text {hypo }}\left(\mathfrak{h}_{0}\right) \chi_{\mathbf{a}}\right)_{L^{2}\left(\left\{\mathbf{x}_{1}, \ldots, \mathbf{x}_{m}\right\} \times \mathbb{R}\right)}
$$

with

$$
\left.\mathbf{K}_{\mathbf{t}, \text { ext }}^{\text {hypo(hol }}\left(\mathbf{h}_{i}, \cdot ; \mathbf{x}_{j}, \cdot\right)=-e^{\left(\mathbf{x}_{j}-\mathbf{x}_{i}\right) \partial^{2}} \mathbf{1}_{\mathbf{x}_{i}<\mathbf{x}_{j}}+\left(\mathbf{S}_{\mathbf{t},-\mathbf{x}_{i}}^{\text {hypo(hol }}\right)^{-}\right) \mathbf{S}_{\mathbf{t}, \mathbf{x}_{j}} \cdot
$$

Our computations here only give pointwise convergence to each of the factors in (3.22); even pointwise convergence of the kernels does not follow, as there is an integration in the middle of $\left(\mathbf{S}_{\mathbf{t},-\mathbf{x}_{i}}^{\left.\text {hypo } \mathfrak{h}_{0}^{-}\right)}\right)^{*} \mathbf{S}_{\mathbf{t}, \mathbf{x}_{j}}$. In Appendix B we prove that the operators actually converge in trace class, which yields convergence of the Fredholm determinants.

Remark 3.7. A remarkable thing has happened in the limiting operation, showing how non-trivial the limit is. From the biorthogonality condition, the 1-point TASEP kernel $K_{t}(n, \cdot ; n, \cdot)$ for any initial data is easily seen to be a projection. This property is lost in the limit; $\mathbf{K}_{\mathbf{t}, \text { ext }}^{\text {hypo(ho }}{ }^{(\mathbf{x}, \cdot ; \mathbf{x}, \cdot)}$ ) is not a projection in general. In the special case of narrow wedge, it is. But for typical examples, such as half-flat, or flat (with the 2-sided formula to appear) it is readily checked that it is not a projection. 


\subsection{From 1-sided to 2 -sided formulas}

The formula for the KPZ fixed point with general initial data $\mathfrak{h}_{0}$ is obtained in the $L \rightarrow \infty$ limit of the formula with truncated initial data $\mathfrak{h}_{0}^{L}(\mathbf{x})=\mathfrak{h}_{0}(\mathbf{x}) \mathbf{1}_{\mathbf{x} \leqslant L}-\infty \cdot \mathbf{1}_{\mathbf{x}>L}$, which is derived from the previous proposition by translation invariance. The fact that the $L \rightarrow \infty$ and $\varepsilon \rightarrow 0$ limits commute follows from the fact that the bound in Lemma 3.4 is independent of $\varepsilon>0$.

Given any function $\mathfrak{g}$, we write

$$
\mathfrak{g}^{ \pm}(\mathbf{y})=\mathfrak{g}( \pm \mathbf{y})
$$

for $\mathbf{y} \geqslant 0$. The shift invariance of TASEP, (2.33), tells us that

$$
\mathfrak{h}\left(\mathbf{t}, \mathbf{x} ; \mathfrak{h}_{0}^{L}\right) \stackrel{\text { dist }}{=} \mathfrak{h}\left(\mathbf{t}, \mathbf{x}-L ; \theta_{L} \mathfrak{h}_{0}^{L}\right),
$$

where $\theta_{L}$ is the shift operator from (2.32), extended to real $L$. With these shifts, Proposition 3.6 tells us that, for UC cutoff data $\mathfrak{h}_{0}^{\varepsilon, L}$ (see Definition 3.3),

$$
\begin{aligned}
\lim _{\varepsilon \rightarrow 0} \mathbb{P}_{\mathfrak{h}_{0}^{\varepsilon, L}}\left(\mathfrak{h}^{\varepsilon}\left(\mathbf{t}, \mathbf{x}_{1}\right) \leqslant \mathbf{a}_{1}, \ldots, \mathfrak{h}^{\varepsilon}\left(\mathbf{t}, \mathbf{x}_{m}\right) \leqslant \mathbf{a}_{m}\right) \\
=\operatorname{det}\left(\mathbf{I}-\chi_{\mathbf{a}} \widetilde{\mathbf{K}}_{L, \text { ext }}^{\theta_{L} \mathfrak{h}_{0}^{L}} \chi_{\mathbf{a}}\right)_{L^{2}\left(\left\{\mathbf{x}_{1}, \ldots, \mathbf{x}_{m}\right\} \times \mathbb{R}\right)},
\end{aligned}
$$

with

$$
\widetilde{\mathbf{K}}_{L, \text { ext }}^{\theta_{L} \mathfrak{h}_{0}^{L}}\left(\mathbf{x}_{i}, \cdot ; \mathbf{x}_{j}, \cdot\right)=-e^{\left(\mathbf{x}_{j}-\mathbf{x}_{i}\right) \partial^{2}} \mathbf{1}_{\mathbf{x}_{i}<\mathbf{x}_{j}}+\left(\mathbf{S}_{\mathbf{t},-\mathbf{x}_{i}+L}^{\mathrm{hypo}\left(\left(\theta_{L} \mathfrak{h}_{0}^{L}\right)^{-}\right)}\right)^{*} \mathbf{S}_{\mathbf{t}, \mathbf{x}_{j}-L}
$$

Use (3.9) to write the second term as

$$
e^{\mathbf{x}_{i} \partial^{2}}\left(\left(\mathbf{S}_{\mathbf{t}, L}^{\mathrm{hypo}\left(\left(\theta_{L} \mathfrak{h}_{0}^{L}\right)^{-}\right)}\right)^{*} \mathbf{S}_{\mathbf{t},-L}\right) e^{-\mathbf{x}_{j} \partial^{2}} .
$$

Since $\left(\theta_{L} \mathfrak{h}_{0}^{L}\right)^{+}(\mathbf{y})=-\infty$ for all $\mathbf{y} \geqslant 0$, we have $\mathbf{S}_{\mathbf{t},-L}^{\text {hypo }\left(\left(\theta_{L} \mathfrak{h}_{0}^{L}\right)^{+}\right)} \equiv 0$, and then we may rewrite $\left(\mathbf{S}_{\mathbf{t}, L}^{\text {hypo }}\left(\left(\theta_{L} \mathfrak{h}_{0}^{L}\right)^{-}\right)\right)^{*} \mathbf{S}_{\mathbf{t},-L}$ as

$$
\mathbf{I}-\left(\mathbf{S}_{\mathbf{t}, L}-\mathbf{S}_{\mathbf{t}, L}^{\mathrm{hypo}\left(\left(\theta_{L} \mathfrak{h}_{0}^{L}\right)^{-}\right)}\right)^{*}\left(\mathbf{S}_{\mathbf{t},-L}-\mathbf{S}_{\mathbf{t},-L}^{\mathrm{hypo}\left(\left(\theta_{L} \mathfrak{h}_{0}^{L}\right)^{+}\right)}\right) .
$$

The crucial fact, first discovered in [51], is that the last expression depends on $L$ only through $\mathfrak{h}_{0}^{L}$, and it actually equals $\mathbf{K}_{\mathbf{t}}^{\text {hypo }\left(\mathfrak{h}_{0}^{L}\right)}$ with

$$
\mathbf{K}_{\mathbf{t}}^{\text {hypo }\left(\mathfrak{h}_{0}\right)}=\mathbf{I}-\left(\mathbf{S}_{\mathbf{t}, 0}-\mathbf{S}_{\mathbf{t}, 0}^{\text {hypo }\left(\mathfrak{h}_{0}^{-}\right)}\right)^{*}\left(\mathbf{S}_{\mathbf{t}, 0}-\mathbf{S}_{\mathbf{t}, 0}^{\text {hypo }\left(\mathfrak{h}_{0}^{+}\right)}\right),
$$

see (4.2) below and the proof sketch that follows it (note also that in this formula the function $\mathfrak{h}_{0}^{+}$appearing in the last operator no longer needs to be truncated in any way). 
Furthermore, it was shown in [51] (for a more restricted class of $\mathfrak{h}_{0}$ ) that we can take $L \rightarrow \infty$ on $\mathbf{K}_{\mathbf{t}}^{\text {hypo }\left(\mathfrak{h}_{0}^{L}\right)}$. More precisely, and in the context of the initial data $\mathfrak{h}_{0} \in \mathrm{UC}$ of the present paper, since $\mathfrak{h}_{0}^{L} \rightarrow \mathfrak{h}_{0}$ in UC, by Theorem 4.1 this kernel converges in trace norm

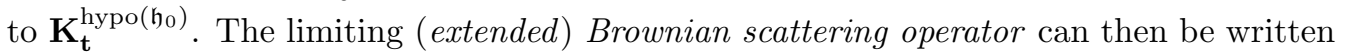
as $\left({ }^{20}\right)$

$$
\mathbf{K}_{\mathbf{t}, \text { ext }}^{\text {hypo }\left(\mathfrak{h}_{0}\right)}=-e^{\left(\mathbf{x}_{j}-\mathbf{x}_{i}\right) \partial^{2}} \mathbf{1}_{\mathbf{x}_{i}<\mathbf{x}_{j}}+e^{-\mathbf{x}_{i} \partial^{2}} \mathbf{K}_{\mathbf{t}}^{\text {hypo }\left(\mathfrak{h}_{0}\right)} e^{\mathbf{x}_{j} \partial^{2}} .
$$

We sometimes also refer to the 1-point kernel (3.25) as the Brownian scattering operator since it is clear how to obtain one from the other. As we mentioned, by Lemma 3.4 we can interchange limits on the left-hand side of (3.23). So, we have shown the following result.

THEOREM 3.8. Let $\mathfrak{h}_{0} \in \mathrm{UC}$ and let $\mathfrak{h}_{0}^{\varepsilon}$ be rescaled TASEP height functions converging to $\mathfrak{h}_{0}$ in UC. Let $y \mathbf{x}_{1}, \ldots, \mathbf{x}_{m}, \mathbf{a}_{1}, \ldots, \mathbf{a}_{m} \in \mathbb{R}$. Then,

$$
\begin{aligned}
\lim _{\varepsilon \rightarrow 0} \mathbb{P}_{\mathfrak{h}_{0}^{\varepsilon}}\left(\mathfrak{h}^{\varepsilon}\left(\mathbf{t}, \mathbf{x}_{1}\right) \leqslant \mathbf{a}_{1}, \ldots, \mathfrak{h}^{\varepsilon}\left(\mathbf{t}, \mathbf{x}_{m}\right) \leqslant \mathbf{a}_{m}\right) \\
=\operatorname{det}\left(\mathbf{I}-\chi_{\mathbf{a}} \mathbf{K}_{\mathbf{t}, \text { ext }}^{\text {hypo }}{ }^{\left.\mathfrak{h}_{0}\right)} \chi_{\mathbf{a}}\right)_{L^{2}\left(\left\{\mathbf{x}_{1}, \ldots, \mathbf{x}_{m}\right\} \times \mathbb{R}\right) .}
\end{aligned}
$$

\subsection{Tightness and Markov property}

The local Hölder spaces $\mathscr{C}_{\bar{\gamma}}^{\beta}$, with $\beta \in\left(0, \frac{1}{2}\right)$ and $\bar{\gamma}<\infty$, defined just after (3.6) are compact subsets of our state space UC.

Theorem 3.9. (Hölder $\frac{1}{2}$-regularity in space) Fix $\mathbf{t}>0, \mathfrak{h}_{0} \in \mathrm{UC}$ and initial data $X_{0}^{\varepsilon}$ for TASEP such that, as $\varepsilon \rightarrow 0, \mathfrak{h}^{\varepsilon}(0, \cdot) \rightarrow \mathfrak{h}_{0}$ in distribution, in UC. Let $\mathfrak{h}^{\varepsilon}(\mathbf{t}, \cdot) \in \mathrm{UC}$ be given by (3.1). Then, for each $\beta \in\left(0, \frac{1}{2}\right)$ and $M<\infty$,

$$
\lim _{A \rightarrow \infty} \limsup _{\varepsilon \rightarrow 0} \mathbb{P}\left(\left\|\mathfrak{h}^{\varepsilon}(\mathbf{t})\right\|_{\beta,[-M, M]} \geqslant A\right)=0 .
$$

Consequently, if $\mathbb{P}_{\varepsilon}$ represents the law of the functions $\mathfrak{h}^{\varepsilon}(\mathbf{t}, \cdot) \in \mathrm{UC}$ given by (3.1), then the family of probability measures $\left\{\mathbb{P}_{\varepsilon}\right\}_{0<\varepsilon<1}$ on $\mathrm{UC}$ is tight (precompact in the topology of weak convergence of measures $)$.

The Hölder regularity (3.28) will be proved in Appendix C.2 using the exact formulas. The method is the Kolmogorov continuity theorem, which reduces regularity to 2-point

$\left({ }^{20}\right)$ There is a slight abuse of notation here, and in our earlier rewriting of the second term of (3.24), when we write $e^{\mathbf{x}_{j} \partial^{2}}$ for $\mathbf{x}_{j}<0$. But note that, in (3.26), $e^{\mathbf{x}_{j} \partial^{2}}$ appears to the right of $\mathbf{K}_{\mathbf{t}}^{\text {hypo(h) }\left(\mathfrak{h}_{0}^{L}\right)}$, and the action of the backwards heat kernel is well defined when applied to the second variable of $\mathbf{K}_{\mathbf{t}}^{\text {hypo }\left(\mathfrak{h}_{0}\right)}$; the same is true for the earlier formula. Whenever we write $\mathbf{K}_{\mathbf{t}}^{\text {hypo(h) }} e^{\mathbf{y} \partial^{2}}$ for $\mathbf{y}<0$, we mean that the backwards heat kernel is applied to this second variable first. In other words, $\mathbf{K}_{\mathbf{t}}^{\mathbf{h y p o}(\mathfrak{h})} e^{\mathbf{y} \partial^{2}}$ is shorthand for the cumbersome $\left(e^{\mathbf{y} \partial^{2}}\left(\mathbf{K}_{\mathbf{t}}^{\text {hypo(h) }}\right)^{*}\right)^{*}$. 
functions, which we can estimate using trace norms following the proof for the Airy process in [48]. To prove the tightness, we need to find compact sets $K_{\delta}$ in UC such that

$$
\limsup _{\delta \rightarrow 0} \limsup _{\varepsilon \rightarrow 0} \mathbb{P}\left(\mathfrak{h}_{\varepsilon}(\mathbf{t}) \notin K_{\delta}\right)=0 .
$$

Since the spaces $\mathscr{C}_{\bar{\gamma}}^{\beta}$ are compact in UC, tightness follows from (3.28) as long as we can show that, if for all $\varepsilon>0, \mathfrak{h}^{\varepsilon}(0, \mathbf{x}) \leqslant \overline{\boldsymbol{\alpha}}+\overline{\boldsymbol{\gamma}}|\mathbf{x}|$ for some $\overline{\boldsymbol{\gamma}}<\infty$ almost surely, then, for some (possibly random) $\bar{\gamma}_{\mathbf{t}}<\infty, \mathfrak{h}^{\varepsilon}(\mathbf{t}, \mathbf{x}) \leqslant \bar{\gamma}_{\mathbf{t}}(1+|\mathbf{x}|)$ for all $\varepsilon>0$ with probability 1 . From the preservation of max property for TASEP (the analog of Theorem 4.5 (vii) below), it suffices to show that

$$
\lim _{A \rightarrow \infty} \limsup _{\varepsilon \rightarrow 0} \mathbb{P}\left(\mathfrak{h}^{\varepsilon}\left(\mathbf{t}, \mathbf{x} ; \bar{\gamma}(1+\mathbf{x}) \mathbf{1}_{\mathbf{x}>0}\right) \leqslant A(1+|\mathbf{x}|) \text { for } \mathbf{x} \in \mathbb{R}\right)=1
$$

Let $\overline{\mathfrak{h}}_{0}^{\varepsilon, B}$ be a rescaled simple asymmetric random walk path with $\overline{\mathfrak{h}}_{0}^{\varepsilon, B}(0)=B$ and $\operatorname{drift} B$. Then,

$$
\lim _{B \rightarrow \infty} \limsup _{\varepsilon \rightarrow 0} \mathbb{P}\left(\overline{\mathfrak{h}}_{0}^{\varepsilon, B}(\mathbf{x}) \geqslant \bar{\gamma}(1+\mathbf{x}) \mathbf{1}_{\mathbf{x}>0} \text { fos } \mathbf{x} \in \mathbb{R}\right)=1 .
$$

Since the asymmetric random walk is invariant, and the drift under rescaling is convergent, the height shift is as well. Therefore,

$$
\lim _{A \rightarrow \infty} \mathbb{P}\left(\mathfrak{h}^{\varepsilon}\left(\mathbf{t}, \mathbf{x} ; \overline{\mathfrak{h}}_{0}^{\varepsilon, B}(\mathbf{x})\right) \leqslant A(1+|\mathbf{x}|) \text { for } \mathbf{x} \in \mathbb{R}\right)=1,
$$

and the result follows from the ordering.

From the above arguments, it follows that any fixed $\mathbf{t}$ distributional limit $\mathfrak{h}(\mathbf{t}, \cdot)$ has finite-dimensional distributions given by the right-hand side of (3.27). In particular, it is unique in distribution. We upgrade to multiple times using the Markov property. However, while one expects the limit of Markov processes to be Markov, this is not always the case. Note that the limiting transition probabilities given by (3.30) are Feller (continuous functions of $\mathfrak{h} \in \mathrm{UC}$ ), by Theorem 4.1 and the fact that $\mathcal{B}_{0}(\mathrm{UC})$, introduced in $\S 3.1$, is a generating family for $\mathcal{B}(\mathrm{UC})$.

Lemma 3.10. Let $\mathscr{P}_{\mathfrak{h}}^{\varepsilon}(t, A)$ be Feller Markov kernels on a Polish space $\mathscr{S}$ for each $\varepsilon>0$, and $\mathscr{P}_{\mathfrak{h}}(t, A)$ be a measurable family of Feller probability kernels on $\mathscr{S}$, such that for each $t>0$ and $\delta>0$ there is a compact subset $K_{\delta}$ of $\mathscr{S}$ such that $\mathscr{P}_{\mathfrak{h}}^{\varepsilon}\left(t, K_{\delta}^{\mathrm{c}}\right)<\delta$, $\mathscr{P}_{\mathfrak{h}}\left(t, K_{\delta}^{\mathrm{c}}\right)<\delta$ and $\lim _{\varepsilon \rightarrow 0} \mathscr{P}_{\mathfrak{h}}^{\varepsilon}(t, A)=\mathscr{P}_{\mathfrak{h}}(t, A)$ uniformly over $\mathfrak{h} \in K_{\delta}$ for each $A$ in a generating family. Then, $\mathscr{P}_{\mathfrak{h}}(t, A)$ satisfies the Chapman-Kolmogorov equations

$$
\int \mathscr{P}_{\mathfrak{h}}(t, d \mathfrak{g}) \mathscr{P}_{\mathfrak{g}}(s, A)=\mathscr{P}_{\mathfrak{h}}(t+s, A) .
$$


Proof. Fix $s, t>0, \mathfrak{h} \in \mathscr{S}, \delta>0$ and $A \in \mathcal{B}(\mathscr{S})$, choose a compact $K_{\delta} \subseteq \mathscr{S}$, and choose $\varepsilon_{0}$ such that, for all $\varepsilon<\varepsilon_{0}, \mathscr{P}_{\mathfrak{h}}^{\varepsilon}\left(t, K_{\delta}^{\mathfrak{c}}\right)+\mathscr{P}_{\mathfrak{h}}\left(t, K_{\delta}^{\mathfrak{c}}\right)<\frac{1}{3} \delta,\left|\mathscr{P}_{\mathfrak{g}}^{\varepsilon}(s, A)-\mathscr{P}_{\mathfrak{g}}(s, A)\right|<\frac{1}{3} \delta$ for all $\mathfrak{g} \in K_{\delta}$, and $\left|\int_{\mathscr{S}}\left(\mathscr{P}_{\mathfrak{h}}^{\varepsilon}(t, d \mathfrak{g})-\mathscr{P}_{\mathfrak{h}}(t, d \mathfrak{g})\right) \mathscr{P}_{\mathfrak{g}}(s, A)\right|<\frac{1}{3} \delta$. Then,

$$
\int_{\mathscr{S}}\left[\mathscr{P}_{\mathfrak{h}}^{\varepsilon}(t, d y) \mathscr{P}_{\mathfrak{g}}^{\varepsilon}(s, A)-\mathscr{P}_{\mathfrak{h}}(t, d \mathfrak{g}) \mathscr{P}_{\mathfrak{g}}(s, A)\right]
$$

is bounded in absolute value by $\mathscr{P}_{\mathfrak{h}}^{\varepsilon}\left(t, K_{\delta}^{\mathfrak{c}}\right)+\mathscr{P}_{\mathfrak{h}}\left(t, K_{\delta}^{\mathfrak{c}}\right)$ plus

$$
\left|\int_{K_{\delta}} \mathscr{P}_{\mathfrak{h}}^{\varepsilon}(t, d \mathfrak{g})\left(\mathscr{P}_{\mathfrak{g}}^{\varepsilon}(s, A)-\mathscr{P}_{\mathfrak{g}}(s, A)\right)\right|+\left|\int_{\mathscr{S}}\left(\mathscr{P}_{\mathfrak{h}}^{\varepsilon}(t, d \mathfrak{g})-\mathscr{P}_{x}(t, d \mathfrak{g})\right) \mathscr{P}_{\mathfrak{g}}(s, A)\right|
$$

all three of which are $<\frac{1}{3} \delta$.

In Appendix B we will show the following result.

Proposition 3.11. The convergence in Theorem 3.8 is uniform over initial data $\mathfrak{h}^{\varepsilon}(0, \cdot)$ in sets of locally bounded Hölder $\beta$ norm, $\beta \in\left(0, \frac{1}{2}\right)$.

As a consequence, we can make the following definition.

Definition 3.12. (KPZ fixed point) The KPZ fixed point is the (unique) Markov process taking values on $\mathrm{UC}$ with transition probabilities given by the extension from the cylindrical subalgebra $\mathcal{B}_{0}(\mathrm{UC})$ to the Borel sets $\mathcal{B}(\mathrm{UC})$ (see $\S 3.1$ ) of

$$
\mathbb{P}_{\mathfrak{h}_{0}}\left(\mathfrak{h}\left(\mathbf{t}, \mathbf{x}_{1}\right) \leqslant \mathbf{a}_{1}, \ldots, \mathfrak{h}\left(\mathbf{t}, \mathbf{x}_{m}\right) \leqslant \mathbf{a}_{m}\right)=\operatorname{det}\left(\mathbf{I}-\chi_{\mathbf{a}} \mathbf{K}_{\mathbf{t}, \operatorname{ext}}^{\mathrm{hypo}\left(\mathfrak{h}_{0}\right)} \chi_{\mathbf{a}}\right)_{L^{2}\left(\left\{\mathbf{x}_{1}, \ldots, \mathbf{x}_{m}\right\} \times \mathbb{R}\right)}
$$

The transition probabilities are Feller.

In the next section we describe its properties. We complete this section by recording the statement we have obtained about the convergence of TASEP to this process from Theorem 3.8, Lemma 3.10 and Proposition 3.11.

THEOREM 3.13. (Convergence of TASEP) Let $\mathfrak{h}^{\varepsilon}(\mathbf{t}, \mathbf{x})$ be the rescaled height function of TASEP given by (3.1). Let $\mathfrak{h}_{0}$ be a element of UC. Assume that we have initial data for TASEP chosen to depend on $\varepsilon>0$ in such a way that $\mathfrak{h}^{\varepsilon}(0, \mathbf{x}) \rightarrow \mathfrak{h}_{0}(\mathbf{x})$ in UC as $\varepsilon \rightarrow 0$. Then, for each $0<\mathbf{t}_{1}<\ldots<\mathbf{t}_{m}$, the rescaled (multi-time) TASEP height function $\left(\mathfrak{h}^{\varepsilon}\left(\mathbf{t}_{1}, \cdot\right), \ldots, \mathfrak{h}^{\varepsilon}\left(\mathbf{t}_{m}, \cdot\right)\right)$ converges in distribution in $\mathrm{UC}^{m}$ to the (multi-time) KPZ fixed point $\left(\mathfrak{h}\left(\mathbf{t}_{1}, \cdot\right), \ldots, \mathfrak{h}\left(\mathbf{t}_{m}, \cdot\right)\right)$ with initial condition $\mathfrak{h}(0, \cdot)=\mathfrak{h}_{0}$. The initial data can be random, and converging in distribution in UC, provided that it is independent of the randomness used to evolve each Markov process. 


\section{The invariant Markov process}

\subsection{Brownian scattering theory}

For height functions $\mathfrak{h}$ in our state space UC of upper semi-continuous functions (see $\S 3.1$ ), define local "hit" and "no-hit" operators by

$$
\mathbf{P}_{\ell_{1}, \ell_{2}}^{\text {no-hit h }}\left(u_{1}, u_{2}\right) d u_{2}=\mathbb{P}_{\mathbf{B}\left(\ell_{1}\right)=u_{1}}\left(\mathbf{B}(\mathbf{y})>\mathfrak{h}(\mathbf{y}) \text { on }\left[\ell_{1}, \ell_{2}\right], \mathbf{B}\left(\ell_{2}\right) \in d u_{2}\right)
$$

and

$$
\mathbf{P}_{\ell_{1}, \ell_{2}}^{\text {hit } \mathfrak{h}}=\mathbf{I}-\mathbf{P}_{\ell_{1}, \ell_{2}}^{\text {no-hit h }}
$$

where $\mathbf{B}$ is a Brownian motion with diffusion coefficient 2 .

The Brownian scattering transform is the map which takes $\mathfrak{h}$ to the $\mathbf{t}>0$ dependent operator (acting on suitable subspaces of $L^{2}(\mathbb{R})$ ) introduced in (3.25), which can be written

$$
\mathbf{K}_{\mathbf{t}}^{\text {hypo(h) }}=\lim _{\substack{\ell_{1} \rightarrow-\infty \\ \ell_{2} \rightarrow \infty}}\left(\mathbf{S}_{\mathbf{t}, \ell_{1}}\right)^{*} \mathbf{P}_{\ell_{1}, \ell_{2}}^{\text {hit } \mathfrak{t}} \mathbf{S}_{\mathbf{t},-\ell_{2}},
$$

where $\mathbf{S}_{\mathbf{t}, \mathbf{x}}=\exp \left\{\mathbf{x} \partial^{2}+\frac{1}{3} \mathbf{t} \partial^{3}\right\}$ are defined by the kernels in (3.10). Before the limit, the right-hand side of (4.1) is exactly $\mathbf{K}_{\mathbf{t}}^{\text {hypo }\left(\mathfrak{h}_{\ell_{1}, \ell_{2}}\right)}$, where $\mathfrak{h}_{\ell_{1}, \ell_{2}}$ is $\mathfrak{h}$ in $\left[\ell_{1}, \ell_{2}\right]$ and $-\infty$ otherwise. The existence of the limit was first proved for a more restricted class in [51]. Since $\mathfrak{h}_{\ell_{1}, \ell_{2}} \rightarrow \mathfrak{h}$ in UC, the limit in (4.1) follows from Theorem 4.1 below, $\left({ }^{21}\right)$ in trace class in $L^{2}([\mathbf{a}, \infty))$ for any fixed $\mathbf{a} \in \mathbb{R}$.

It was proved in [51] that the limit can also be represented as $\left({ }^{22}\right),(23)$

$$
\left.\mathbf{K}_{\mathbf{t}}^{\text {hypo(h) }}=\left(\mathbf{S}_{\mathbf{t}, \mathbf{x}}^{\text {hypo }\left(\mathfrak{h}^{\mathbf{x},-}\right)}\right)^{*} \mathbf{S}_{\mathbf{t},-\mathbf{x}}+\left(\mathbf{S}_{\mathbf{t}, \mathbf{x}}\right)^{*} \mathbf{S}_{\mathbf{t},-\mathbf{x}}^{\text {hypo(h }} \mathfrak{h}^{\mathbf{x},+}\right)-\left(\mathbf{S}_{\mathbf{t}, \mathbf{x}}^{\text {hypo }\left(\mathfrak{h}^{\mathbf{x},-}\right)}\right)^{*} \mathbf{S}_{\mathbf{t},-\mathbf{x}}^{\text {hypo( } \left.\mathfrak{h}^{\mathbf{x},+}\right)}
$$

for any choice of splitting point $\mathbf{x}$, where

$$
\mathfrak{h}^{\mathbf{x}, \pm}(\mathbf{y})=\mathfrak{h}(\mathbf{x} \pm \mathbf{y})
$$

$\left({ }^{21}\right)$ Theorem 4.1 also asks for the kernel to be conjugated by $\vartheta$, but as can be seen from the arguments in Appendix A, this is not necessary for the single-time kernel.

$\left({ }^{22}\right)$ The derivation in [51] takes a different route, starting with known path integral kernel formulas for the Airy 2 process and passing to limits. These known formulas themselves arise from the exact TASEP formulas for step initial data. Here we have started from general initial data and derived $\mathbf{K}_{\mathbf{t}}^{\text {hypo(h) }}$ in a multi-point formula at a later time. Specializing the present derivation to the 1-point case, the two routes are linked through time inversion, as explained around (2.12).

$\left.{ }^{23}\right)$ [51] works with the epi version of $\mathbf{K}_{\mathbf{t}}^{\text {hypo(h) }}$, which is defined by considering the hitting probabilities of the epigraph of a lower semicontinuous function (see (3.13)), and only in the case $\mathbf{t}=1$, but the proof can be adapted straightforwardly. That paper also works under an additional regularity assumption on the barrier function; the more general setting which we work with here can be handled as in Appendix A. 
for $\mathbf{y} \geqslant 0$; the case $\mathbf{x}=0$ is just a rewriting of (3.25), while in [51] it is shown that the right-hand side does not depend on $\mathbf{x}$. We sketch the idea:

$$
\mathbf{P}_{\ell_{1}, \ell_{2}}^{\text {no-hit hy }}\left(u_{1}, u_{2}\right)=p_{\ell_{1}, u_{1}}\left(\ell_{2}, u_{2}\right) p_{\ell_{1}, u_{1}, \ell_{2}, u_{2}} \text { (no hit), }
$$

where $p_{\ell_{1}, u_{1}}\left(\ell_{2}, u_{2}\right)$ is the transition density for Brownian motion to be at $u_{2}$ at time $\ell_{2}$ given that it started at $u_{1}$ at time $\ell_{1}$, and where the second factor is the probability for a Brownian bridge with the same endpoints not to hit hypo $(\mathfrak{h})$, which we can write for $\mathbf{x} \in\left[\ell_{1}, \ell_{2}\right]$ as

$$
\int_{\mathfrak{h}(\mathbf{x})}^{\infty} p_{\ell_{1}, u_{1}, \ell_{2}, u_{2}}(\mathbf{B}(\mathbf{x}) \in d z) p_{0, z, \mathbf{x}-\ell_{1}, u_{1}}\left(\text { no hit } \mathfrak{h}^{\mathbf{x},-} \text { on }\left[0, \mathbf{x}-\ell_{1}\right]\right) p_{0, z, \ell_{2}-\mathbf{x}, u_{2}}\left(\text { no hit } \mathfrak{h}^{\mathbf{x},+} \text { on }\left[0, \ell_{2}-\mathbf{x}\right]\right) \text {. }
$$

Now,

$$
p_{\ell_{1}, u_{1}}\left(\ell_{2}, u_{2}\right) p_{\ell_{1}, u_{1}, \ell_{2}, u_{2}}(\mathbf{B}(\mathbf{x}) \in d z)=p_{0, z}\left(\mathbf{x}-\ell_{1}, u_{1}\right) p_{0, z}\left(\ell_{2}-\mathbf{x}, u_{2}\right) d z,
$$

so (4.4) becomes

$$
\int_{\mathfrak{h}(\mathbf{x})}^{\infty} d z p_{0, z}\left(\text { no hit } \mathfrak{h}^{\mathbf{x},-} \text { on }\left[0, \mathbf{x}-\ell_{1}\right], \mathbf{x}-\ell_{1}, u_{1}\right) p_{0, z}\left(\text { no hit } \mathfrak{h}^{\mathbf{x},+} \text { on }\left[0, \ell_{2}-\mathbf{x}\right], \ell_{2}-\mathbf{x}, u_{2}\right),
$$

where the notation is meant to indicate that the factors in the integrand are now densities. Again, we can write $p_{0, z}$ (no hit $\mathfrak{h}$ on $[0, \ell], \ell, u$ ) as

$$
p_{0, z}(\ell, u)-p_{0, z}(\text { hit } \mathfrak{h} \text { on }[0, \ell], \ell, u),
$$

which is just

$$
e^{\ell \partial^{2}}(z, u)-\int_{0}^{\ell} p_{z}(\boldsymbol{\tau} \in d \mathbf{s}) e^{(\ell-\mathbf{s}) \partial^{2}}(\mathbf{B}(\boldsymbol{\tau}), u) .
$$

Therefore, the right-hand side of (4.1) goes to the right-hand side of (4.2) as $\ell_{1} \rightarrow \infty$ and $\ell_{2} \rightarrow \infty$.

In (3.26) we defined the extended version of the Brownian scattering transform, which, in view of (4.2), can be written

$$
\begin{aligned}
\mathbf{K}_{\mathbf{t}, \text { ext }}^{\text {hypo }(\mathfrak{h})}=- & e^{\left(\mathbf{x}_{j}-\mathbf{x}_{i}\right) \partial^{2}} \mathbf{1}_{\mathbf{x}_{i}<\mathbf{x}_{j}}+\left(\mathbf{S}_{\mathbf{t}, \mathbf{x}-\mathbf{x}_{i}}^{\mathrm{hypo}\left(\mathfrak{h}^{\mathbf{x},-}\right)}\right)^{*} \mathbf{S}_{\mathbf{t},-\mathbf{x}+\mathbf{x}_{j}} \\
& +\left(\mathbf{S}_{\mathbf{t}, \mathbf{x}-\mathbf{x}_{i}}\right)^{*} \mathbf{S}_{\mathbf{t},-\mathbf{x}+\mathbf{x}_{j}}^{\text {hypo }\left(\mathfrak{h}^{\mathbf{x},+}\right)}-\left(\mathbf{S}_{\mathbf{t}, \mathbf{x}-\mathbf{x}_{i}}^{\text {hypo( }}\right)^{*} \mathbf{S}_{\mathbf{t},-\mathbf{x}+\mathbf{x}_{j}}^{\text {hypo }\left(\mathfrak{h}^{\mathbf{x},+}\right)} .
\end{aligned}
$$

For fixed $\mathbf{t}$, and after conjugating by

$$
\vartheta f\left(\mathbf{x}_{i}, u\right)=\vartheta_{i}(u) f\left(\mathbf{x}_{i}, u\right), \quad \text { with } \vartheta_{i}(u)=\left(1+u^{2}\right)^{2 i},
$$

and cutting off by $\chi_{\mathbf{a}}$ (see (2.4)), the Brownian scattering transform is continuous on $\mathrm{UC}$, as we state in the following theorem. 
Theorem 4.1. For fixed $\mathbf{a}_{1}, \ldots, \mathbf{a}_{m}>-\infty$ and $\mathbf{t}>0$, we have that

$$
\mathfrak{h} \longmapsto \vartheta \chi_{\mathbf{a}} \mathbf{K}_{\mathbf{t}, \text { ext }}^{\text {hypo(h) }} \chi_{\mathbf{a}} \vartheta^{-1}
$$

is a continuous map from $\mathrm{UC}$ into the trace class operators on $L^{2}\left(\left\{\mathbf{x}_{1}, \ldots, \mathbf{x}_{m}\right\} \times \mathbb{R}\right)$.

Moreover, a UC function $\mathfrak{h}$ can be recovered from its Brownian scattering transform $\mathbf{K}_{\mathbf{t}, \operatorname{ext}}^{\text {hypo(h) }}$.

Theorem 4.2. (Inversion formula) For any $\mathfrak{h} \in \mathrm{UC}$,

$$
\lim _{\mathbf{t} \backslash 0} \operatorname{det}\left(\mathbf{I}-\chi_{\mathbf{a}} \mathbf{K}_{\mathbf{t}, \text { ext }}^{\text {hypo(h) }} \chi_{\mathbf{a}}\right)_{L^{2}\left(\left\{\mathbf{x}_{1}, \ldots, \mathbf{x}_{m}\right\} \times \mathbb{R}\right)}=\prod_{j=1}^{m} \mathbf{1}_{\mathfrak{h}\left(\mathbf{x}_{j}\right) \leqslant \mathbf{a}_{j}} .
$$

The fact that the kernel appearing in Theorem 4.1 is trace class will be proved in Appendix A; the continuity stated in the result follows from the arguments in Appendix B.2. Theorem 4.2 follows directly from the Chapman-Kolmogorov equations (3.29).

\subsection{KPZ fixed point formula}

The Brownian scattering transform linearizes the time evolution of the fixed point transition probabilities: at the level of Brownian scattering operators, the time flow is linear, satisfying the Lax equation

$$
\partial_{\mathbf{t}} \mathbf{K}_{\mathbf{t}, \text { ext }}^{\text {hypo(h) }}=\left[-\frac{1}{3} \partial^{3}, \mathbf{K}_{\mathbf{t}, \text { ext }}^{\mathrm{hypo}(\mathfrak{h})}\right] .
$$

As shown in $\S 3$, the Fredholm determinant maps this linear flow to the Markov transition probabilities given by the KPZ fixed point formula

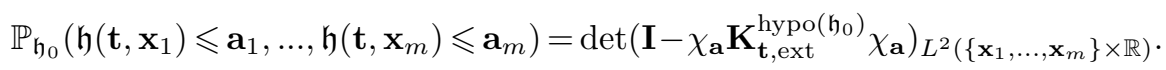

The resulting Markov process, the KPZ fixed point (see Definition 3.12), is thus a stochastic integrable system in the sense discussed in the TASEP case (§2.4).

As for TASEP, we also have a version of the fixed point formula in terms of the Fredholm determinant of a kernel acting on $L^{2}(\mathbb{R})$ ) (as opposed to an "extended kernel" acting on $\left.L^{2}\left(\left\{\mathbf{x}_{1}, \ldots, \mathbf{x}_{m}\right\} \times \mathbb{R}\right)\right)$.

Proposition 4.3. (Path integral formula for the KPZ fixed point) Given $\mathfrak{h}_{0} \in \mathrm{UC}$, $\mathbf{t}>0$, and $\mathbf{x}_{1}<\ldots<\mathbf{x}_{m}$, we have

$$
\begin{aligned}
& \mathbb{P}_{\mathfrak{h}_{0}}\left(\mathfrak{h}\left(\mathbf{t}, \mathbf{x}_{1}\right) \leqslant \mathbf{a}_{1}, \ldots, \mathfrak{h}\left(\mathbf{t}, \mathbf{x}_{m}\right) \leqslant \mathbf{a}_{m}\right) \\
& =\operatorname{det}\left(\mathbf{I}-\mathbf{K}_{\mathbf{t}, \mathbf{x}_{1}}^{\text {hypo(} \left.\mathfrak{h}_{0}\right)}+\bar{\chi}_{\mathbf{a}_{1}} e^{\left(\mathbf{x}_{2}-\mathbf{x}_{1}\right) \partial^{2}} \bar{\chi}_{\mathbf{a}_{2}} \ldots e^{\left(\mathbf{x}_{m}-\mathbf{x}_{m-1}\right) \partial^{2}} \bar{\chi} \overline{\mathbf{a}}_{m} e^{\left(\mathbf{x}_{1}-\mathbf{x}_{m}\right) \partial^{2}} \mathbf{K}_{\mathbf{t}, \mathbf{x}_{1}}^{\mathrm{hypo}\left(\mathfrak{h}_{0}\right)}\right)_{L^{2}(\mathbb{R})},
\end{aligned}
$$

where $\mathbf{K}_{\mathbf{t}, \mathbf{x}}^{\text {hypo(ho }}(\cdot, \cdot)=\mathbf{K}_{\mathbf{t}, \text { ext }}^{\text {hypo }}\left(\mathfrak{h}_{0}\right)(\mathbf{x}, \cdot, \mathbf{x}, \cdot)$. 
This results from an application of [8, Theorem 3.3], and is proved in Appendix D.1. Taking a continuum limit gives a very symmetric version of the fixed point formula, from which the skew time reversal symmetry, Theorem 4.5 (iii), follows by the cyclicity of the determinant.

Proposition 4.4. (Continuum statistics) For any $\mathfrak{h}_{0} \in \mathrm{UC}, \mathfrak{g} \in \mathrm{LC}$ and $\mathbf{t}>0$,

$$
\mathbb{P}_{\mathfrak{h}_{0}}(\mathfrak{h}(\mathbf{t}, \mathbf{x}) \leqslant \mathfrak{g}(\mathbf{x}) \text { for } \mathbf{x} \in \mathbb{R})=\operatorname{det}\left(\mathbf{I}-\mathbf{K}_{\mathbf{t} / 2}^{\mathrm{hypo}\left(\mathfrak{h}_{0}\right)} \mathbf{K}_{-\mathbf{t} / 2}^{\mathrm{epi}(\mathfrak{g})}\right)_{L^{2}(\mathbb{R})},
$$

with $\mathbf{K}_{-\mathbf{t}}^{\mathrm{epi}(\mathfrak{g})}(u, v)=\mathbf{K}_{\mathbf{t}}^{\mathrm{hypo}(-\mathfrak{g})}(-u,-v)$.

We have that $\mathbf{K}_{-\mathbf{t}}^{\mathrm{epi}(\mathfrak{g})}$ is just an upside-down version of the Brownian scattering transform introduced in (4.2), and is built in an analogous way out of hitting probabilities of the epigraph of lower semicontinuous functions (replacing $\mathbf{S}_{\mathbf{t}, \mathbf{x}}^{\text {hypo(h) }}$ by $\mathbf{S}_{-\mathbf{t}, \mathbf{x}}^{\mathrm{epi}(\mathfrak{g})}$, which was defined in (3.13).) In Appendix A, we show that the operator inside the above Fredholm determinant is trace class after an appropriate conjugation.

Proof. Consider first $\mathfrak{g} \in \mathrm{LC}$ which is $\infty$ outside of some interval $[-R, R]$. The lefthand side of (4.9) is then $\mathbb{P}_{\mathfrak{h}_{0}}(\mathfrak{h}(\mathbf{t}, \mathbf{x}) \leqslant \mathfrak{g}(\mathbf{x})$ for $\mathbf{x} \in[-R, R])$, which we may obtain by computing $\mathbb{P}_{\mathfrak{h}_{0}}\left(\mathfrak{h}\left(\mathbf{t}, \mathbf{x}_{i}\right) \leqslant \mathfrak{g}\left(\mathbf{x}_{i}\right)\right.$ for $\left.i=1, \ldots, m\right)$ on a mesh $\mathbf{x}_{1}<\ldots<\mathbf{x}_{m}$ of $[-R, R]$ and letting the mesh size go to zero as $m \rightarrow \infty$. To this end, we use Proposition 4.3 with $\mathbf{a}_{i}=\mathfrak{g}\left(\mathbf{x}_{i}\right)$. Since $\mathbf{S}_{\mathbf{t} / 2, \mathbf{x}_{1}} \mathbf{K}_{\mathbf{t}, \mathbf{x}_{1}}^{\text {hypo(holo })}\left(\mathbf{S}_{\mathbf{t} / 2,-\mathbf{x}_{1}}\right)^{*}=\mathbf{K}_{\mathbf{t} / 2}^{\text {hypo(hol }}{ }^{\left(\mathfrak{h}_{0}\right)}$, we can conjugate the kernel inside the determinant in (4.8) by $\mathbf{S}_{\mathbf{t} / 2, \mathbf{x}_{1}}$, to get

$$
\mathbf{K}_{\mathbf{t} / 2}^{\text {hypo }\left(\mathfrak{h}_{0}\right)}-\left[\mathbf{S}_{\mathbf{t} / 2, \mathbf{x}_{1}} \bar{\chi}_{\mathfrak{g}\left(\mathbf{x}_{1}\right)} e^{\left(\mathbf{x}_{2}-\mathbf{x}_{1}\right) \partial^{2}} \bar{\chi}_{\mathfrak{g}\left(\mathbf{x}_{2}\right)} \ldots e^{\left(\mathbf{x}_{m}-\mathbf{x}_{m-1}\right) \partial^{2}} \bar{\chi}_{\mathfrak{g}\left(\mathbf{x}_{m}\right)}\left(\mathbf{S}_{\mathbf{t} / 2,-\mathbf{x}_{m}}\right)^{*}\right] \mathbf{K}_{\mathbf{t} / 2}^{\text {hypo }\left(\mathfrak{h}_{0}\right)}
$$

Taking $\mathbf{x}_{1}=-R$ and $\mathbf{x}_{m}=R$, let $\mathfrak{g}^{(m)} \in \mathrm{LC}$ be given as $\mathfrak{g}^{(m)}\left(\mathbf{x}_{i}+R\right)=\mathfrak{g}\left(\mathbf{x}_{i}\right), i=1, \ldots, m$, and $\mathfrak{g}^{(m)}(\mathbf{x})=-\infty$ for all other values of $\mathbf{x}$, and note that

$$
\bar{\chi}_{\mathfrak{g}\left(\mathbf{x}_{1}\right)} e^{\left(\mathbf{x}_{2}-\mathbf{x}_{1}\right) \partial^{2}} \bar{\chi}_{\mathfrak{g}\left(\mathbf{x}_{2}\right)} \ldots e^{\left(\mathbf{x}_{m}-\mathbf{x}_{m-1}\right) \partial^{2}} \bar{\chi}_{\mathfrak{g}\left(\mathbf{x}_{m}\right)}=e^{2 R \partial^{2}}-\mathbf{S}_{0,2 R}^{\mathrm{epi}\left(\mathfrak{g}^{(m)}\right)}
$$

(because the Brownian motion $\mathbf{B}$ inside the epi operator can only hit epi $\left(\mathfrak{g}^{(m)}\right)$ at zero, $\mathbf{x}_{2}-\mathbf{x}_{1}, \ldots, \mathbf{x}_{m}-\mathbf{x}_{1}$, and the left-hand side is simply the transition probability for $\mathbf{B}$ in $[0,2 R]$ staying below the same epigraph). Then, the term in brackets in (4.10) equals

$$
\mathbf{S}_{\mathbf{t} / 2,-R}\left(\mathbf{S}_{0,2 R}-\mathbf{S}_{0,2 R}^{\mathrm{epi}\left(\mathfrak{g}^{(m)}\right)}\right)\left(\mathbf{S}_{\mathbf{t} / 2,-R}\right)^{*}=\left(\mathbf{S}_{-\mathbf{t} / 2,0}\right)^{*}\left(\mathbf{S}_{-\mathbf{t} / 2,0}-\mathbf{S}_{-\mathbf{t} / 2,0}^{\mathrm{epi}\left(\mathfrak{g}^{(m)}\right)}\right)=\mathbf{I}-\mathbf{K}_{-\mathbf{t} / 2}^{\mathrm{epi}\left(\mathfrak{g}^{m}\right)},
$$

where the last equality follows from using the epi version of the expansion (4.2) split at $\mathbf{x}=0$. As a consequence, the right-hand side of (4.7) can be written as

$$
\operatorname{det}\left(\mathbf{I}-\mathbf{K}_{\mathbf{t} / 2}^{\mathrm{hypo}\left(\mathfrak{h}_{0}\right)} \mathbf{K}_{-\mathbf{t} / 2}^{\mathrm{epi}\left(\mathfrak{g}^{(m)}\right)}\right)_{L^{2}(\mathbb{R})}
$$


(after using the cyclic property of the determinant). Since $-\mathfrak{g}^{(m)} \rightarrow-\mathfrak{g}$ in UC, one would like to use Theorem 4.1 to pass to the limit and obtain (4.9). The difficulty is that in this Fredholm determinant we are missing the necessary conjugations, but this is resolved by using (A.3) and the comment that follows it, which implies that the trace-norm estimates of Appendix A are strong enough to yield continuity in exactly the form we need.

In order to extend the result to all $\mathfrak{g} \in \mathrm{LC}$, it is enough to truncate $\mathfrak{g}$ to a function which is $\infty$ outside $[-R, R]$, apply the result we just proved, and then take $R \rightarrow \infty$. The left-hand side clearly converges to $\mathbb{P}_{\mathfrak{h}_{0}}(\mathfrak{h}(\mathbf{t}, \mathbf{x}) \leqslant \mathfrak{g}(\mathbf{x})$ for $\mathbf{x} \in \mathbb{R})$, while the right-hand side converges to the desired Fredholm determinant using again Theorem 4.1 and the same argument as above.

Analogously to Theorem 4.2 we also have the inversion formula

$$
\lim _{\mathbf{t} \searrow 0} \operatorname{det}\left(\mathbf{I}-\mathbf{K}_{\mathbf{t} / 2}^{\text {hypo(h) }} \mathbf{K}_{-\mathbf{t} / 2}^{\mathrm{epi}(\mathfrak{g})}\right)=\mathbf{1}_{\mathfrak{h}(\mathbf{x}) \leqslant \mathfrak{g}(\mathbf{x}) \text { for all } \mathbf{x} \in \mathbb{R} .}
$$

\subsection{Properties of the KPZ fixed point}

The KPZ fixed point satisfies a number of additional properties, which can be proved based both on the explicit formula and on approximation from TASEP.

Theorem 4.5. (Symmetries) Let $\mathfrak{h}\left(\mathbf{t}, \mathbf{x} ; \mathfrak{h}_{0}\right)$ denote the KPZ fixed point with initial data $\mathfrak{h}_{0} \in \mathrm{UC}$.

(i) (1:2:3 scaling invariance) For any $\alpha>0$,

$$
\alpha \mathfrak{h}\left(\alpha^{-3} \mathbf{t}, \alpha^{-2} \mathbf{x} ; \alpha^{-1} \mathfrak{h}_{0}\left(\alpha^{2} \mathbf{x}\right)\right) \stackrel{\text { dist }}{=} \mathfrak{h}\left(\mathbf{t}, \mathbf{x} ; \mathfrak{h}_{0}\right) .
$$

(ii) (Invariance of Brownian motion) If $\mathbf{B}(\mathbf{x})$ is a 2-sided Brownian motion, then, for each $\mathbf{t}>0, \mathfrak{h}(\mathbf{t}, \mathbf{x} ; \mathbf{B})-\mathfrak{h}(\mathbf{t}, 0 ; \mathbf{B})$ is a 2-sided Brownian motion in $\mathbf{x}$ with diffusion coefficient 2 .

(iii) (Skew time reversibility) For any $\mathfrak{f}, \mathfrak{g} \in \mathrm{UC}$,

$$
\mathbb{P}(\mathfrak{h}(\mathbf{t}, \mathbf{x} ; \mathfrak{g}) \leqslant-\mathfrak{f}(\mathbf{x}))=\mathbb{P}(\mathfrak{h}(\mathbf{t}, \mathbf{x} ; \mathfrak{f}) \leqslant-\mathfrak{g}(\mathbf{x})) .
$$

(iv) (Stationarity in space) $\mathfrak{h}\left(\mathbf{t}, \mathbf{x}+\mathbf{u} ; \mathfrak{h}_{0}(\mathbf{x}-\mathbf{u})\right) \stackrel{\text { dist }}{=} \mathfrak{h}\left(\mathbf{t}, \mathbf{x} ; \mathfrak{h}_{0}\right)$.

(v) (Reflection invariance) $\mathfrak{h}\left(\mathbf{t},-\mathbf{x} ; \mathfrak{h}_{0}(-\mathbf{x})\right) \stackrel{\text { dist }}{=} \mathfrak{h}\left(\mathbf{t}, \mathbf{x} ; \mathfrak{h}_{0}\right)$.

(vi) (Affine invariance) $\mathfrak{h}\left(\mathbf{t}, \mathbf{x} ; \mathfrak{h}_{0}(\mathbf{x})+\mathbf{a}+c \mathbf{x}\right) \stackrel{\text { dist }}{=} \mathfrak{h}\left(\mathbf{t}, \mathbf{x}+\frac{1}{2} c \mathbf{t} ; \mathfrak{h}_{0}(\mathbf{x})\right)+\mathbf{a}+c \mathbf{x}+\frac{1}{4} c^{2} \mathbf{t}$.

(vii) (Preservation of max) For any $\mathfrak{f}_{1}, \mathfrak{f}_{2} \in \mathrm{UC}$,

$$
\mathfrak{h}\left(\mathbf{t}, \mathbf{x} ; \mathfrak{f}_{1} \vee \mathfrak{f}_{2}\right) \stackrel{\text { dist }}{=} \mathfrak{h}\left(\mathbf{t}, \mathbf{x} ; \mathfrak{f}_{1}\right) \vee \mathfrak{h}\left(\mathbf{t}, \mathbf{x} ; \mathfrak{f}_{2}\right)
$$


These properties follow from Theorem 3.13; (i) since $\mathfrak{h}$ is a limit and therefore a fixed point of the 1:2:3 rescaling and (ii)-(vii) from the analog properties for TASEP. (iii)-(vi) can alternatively be seen to follow directly from the fixed point formula (4.7) (see also Theorem 4.4 in the case of (iii)), and the affine invariance can also be proved from the variational formul (Theorem 4.18). Note that in (ii) there is a non-trivial global height shift, and the Brownian motion measure itself is not invariant. Combining (ii) and (vi), one sees that drifted Brownian motion $\mathbf{B}(\mathbf{x})+\rho \mathbf{x}$ is also invariant.

Another property which follows by approximation from TASEP (see Lemma 3.4) is the following.

Theorem 4.6. (Finite propagation speed) Let $\mathfrak{h}_{0} \in \mathrm{UC}$ with $\mathfrak{h}_{0}(\mathbf{x}) \leqslant \overline{\boldsymbol{\alpha}}+\overline{\boldsymbol{\gamma}}|\mathbf{x}|$ and let $\mathbf{x}_{1}, \ldots, \mathbf{x}_{m} \in \mathbb{R}$. For any $\delta>0$ there exists $C<\infty$ depending only on $\overline{\boldsymbol{\alpha}}, \overline{\boldsymbol{\gamma}}, L$ and $\max _{i}\left|\mathbf{x}_{i}\right|$, such that, for any $\tilde{\mathfrak{h}}_{0} \in \mathrm{UC}$ with $\tilde{\mathfrak{h}}_{0}(\mathbf{x}) \leqslant \overline{\boldsymbol{\alpha}}+\overline{\boldsymbol{\gamma}}|\mathbf{x}|$ and $\mathfrak{h}_{0}(\mathbf{x})=\tilde{\mathfrak{h}}_{0}(\mathbf{x})$ for $|\mathbf{x}| \leqslant L$,

$$
\mid \mathbb{P}_{\mathfrak{h}_{0}}\left(\mathfrak{h}\left(\mathbf{t}, \mathbf{x}_{i}\right) \leqslant \mathbf{a}_{i} \text { for } i=1, \ldots, m\right)-\mathbb{P}_{\tilde{h}_{0}}\left(\mathfrak{h}\left(\mathbf{t}, \mathbf{x}_{i}\right) \leqslant \mathbf{a}_{i} \text { for } i=1, \ldots, m\right) \mid \leqslant C e^{-(2 / 3-\delta) L^{3}} .
$$

By bounding above and below by known cases, we obtain rather easily the following result. $\left({ }^{24}\right)$

Proposition 4.7. (Tail estimates) Let $\mathfrak{h}_{0} \in \mathrm{UC}, \mathfrak{h}_{0} \not \equiv-\infty$. Then, for fixed $\mathbf{t}>0$, we have

$$
\begin{aligned}
1-e^{-(1 / 12) \mathbf{t}^{-1}\left|\max _{i} \mathbf{a}_{i}\right|^{3}\left(1+o_{1}(1)\right)} & \leqslant \mathbb{P}_{\mathfrak{h}_{0}}\left(\mathfrak{h}\left(\mathbf{t}, \mathbf{x}_{i}\right) \geqslant \mathbf{a}_{i} \text { for } i=1, \ldots, m\right) \\
& \leqslant e^{-(4 / 3) \mathbf{t}^{-1 / 2}\left|\max _{i} \mathbf{a}_{i}\right|^{3 / 2}\left(1+o_{2}(1)\right)},
\end{aligned}
$$

where $o_{1}(1) \rightarrow 0$ as $\max _{i} \mathbf{a}_{i} \rightarrow-\infty, o_{2}(1) \rightarrow 0$ as $\max _{i} \mathbf{a}_{i} \rightarrow \infty$, and both depend only on $\mathfrak{h}_{0}$, $\mathbf{t}$ and the $\mathbf{x}_{i}$ 's.

Proof. Fix $\mathbf{x}_{1}, \ldots, \mathbf{x}_{m} \in \mathbb{R}$ and let

$$
p_{m}\left(\mathbf{a}_{1}, \ldots, \mathbf{a}_{m}\right)=\mathbb{P}_{\mathfrak{h}_{0}}\left(\mathfrak{h}\left(\mathbf{t}, \mathbf{x}_{i}\right) \leqslant \mathbf{a}_{i} \text { for } i=1, \ldots, m\right) .
$$

To see that $p_{m}\left(\mathbf{a}_{1}, \ldots, \mathbf{a}_{m}\right) \rightarrow 0$ at the desired speed as any of the $\mathbf{a}_{i}$ 's goes to $-\infty$, we use the trivial fact that $p_{m}\left(\mathbf{a}_{1}, \ldots, \mathbf{a}_{m}\right) \leqslant p_{1}\left(\mathbf{a}_{i}\right)$ for any $i$. By the skew time reversal symmetry and the affine invariance of the fixed point (Theorem 4.5 ((iii) and (vi)), together with (4.14), we know the 1-dimensional marginals

$$
p_{1}\left(\mathbf{a}_{i}\right)=\mathbb{P}\left(\mathcal{A}_{2}(\mathbf{x})-\left(\mathbf{x}-\mathbf{x}_{i}\right)^{2} \leqslant-\mathfrak{h}_{0}(\mathbf{x})+\mathbf{a}_{i} \text { for } \mathbf{x} \in \mathbb{R}\right),
$$

$\left({ }^{24}\right)$ These estimates are sharp, as $\mathbf{a}_{i} \rightarrow \infty$ (ignoring lower-order terms), and do not depend on the initial data (within UC), but they are not sharp as $\mathbf{a}_{i} \rightarrow-\infty$. In fact, the left tail depends on the initial data; for instance, $\mathbb{P}(\mathfrak{h}(\mathbf{t}, \mathbf{x}) \leqslant \mathbf{a})$ is of order $e^{-(1 / 12) \mathbf{t}^{-1}|\mathbf{a}|^{3}}$ for narrow wedge, but of order $e^{-(1 / 6) \mathbf{t}^{-1}|\mathbf{a}|^{3}}$ for flat. 
where $\mathcal{A}_{2}(\mathbf{x})$ is the Airy 2 process (see $\S 4.4$ ), and we have taken $\mathbf{t}=1$ (general $\mathbf{t}>0$ follows by scaling invariance). Choosing $\overline{\mathbf{x}}$ so that $\mathfrak{h}_{0}(\overline{\mathbf{x}})>-\infty$, we can bound $p_{1}\left(\mathbf{a}_{i}\right)$ by

$$
\mathbb{P}\left(\mathcal{A}_{2}(\overline{\mathbf{x}})-\left(\overline{\mathbf{x}}-\mathbf{x}_{i}\right)^{2} \leqslant-\mathfrak{h}_{0}(\overline{\mathbf{x}})+\mathbf{a}_{i}\right),
$$

which is a shifted $F_{\mathrm{GUE}}$. Hence, we have

$$
p_{m}\left(\mathbf{a}_{1}, \ldots, \mathbf{a}_{m}\right) \lesssim \exp \left\{-\frac{1}{12}\left|\mathbf{a}_{i}\right|^{3}\right\}
$$

as any $\mathbf{a}_{i} \rightarrow-\infty$, proving the lower bound of Proposition 4.7.

To show that $p_{m}\left(\mathbf{a}_{1}, \ldots, \mathbf{a}_{m}\right) \rightarrow 1$ at the desired speed as all $\mathbf{a}_{i} \rightarrow \infty$, one can use (4.7) together with the estimate

$$
|\operatorname{det}(\mathbf{I}-\mathbf{K})-1| \leqslant\|\mathbf{K}\|_{1} e^{\|\mathbf{K}\|_{1}+1}
$$

(with $\|\cdot\|_{1}$ denoting trace norm, see (A.2)). Computing carefully, this gives the desired limit and the upper bound of Proposition 4.7. On the other hand, there is a simple trick using the preservation of max property, Theorem 4.5 (vii) (whose proof is independent), which yields the same estimate. Fix time $\mathbf{t}=1$ again for simplicity. Since

$$
\mathfrak{h}_{0}(\mathbf{x}) \leqslant \bar{\gamma}(1+|x|)
$$

we have by preservation of max that

$$
\mathfrak{h}(1, \mathbf{x}) \stackrel{\text { dist }}{\leqslant} \max \{\mathfrak{h}(1, \mathbf{x} ; \bar{\gamma}(1+x)), \mathfrak{h}(1, \mathbf{x} ; \bar{\gamma}(1-x))\} .
$$

By affine invariance, Theorem 4.5 (vi), we have

$$
\begin{aligned}
& \mathfrak{h}(1, \mathbf{x} ; \bar{\gamma}(1+x)) \stackrel{\text { dist }}{=} \mathfrak{h}\left(1, \mathbf{x}+\frac{1}{2} \bar{\gamma} ; 0\right)+\bar{\gamma}(1+x)+\frac{1}{4} \bar{\gamma}^{2}, \\
& \mathfrak{h}(1, \mathbf{x} ; \bar{\gamma}(1-x)) \stackrel{\text { dist }}{=} \mathfrak{h}\left(1, \mathbf{x}-\frac{1}{2} \bar{\gamma} ; 0\right)+\bar{\gamma}(1-x)+\frac{1}{4} \bar{\gamma}^{2} .
\end{aligned}
$$

So, using (4.15), we get

$$
\begin{aligned}
\mathbb{P}\left(\mathfrak{h}\left(1, \mathbf{x}_{i} ; \mathfrak{h}_{0}\right) \geqslant \mathbf{a}_{i}\right) \leqslant & \mathbb{P}\left(\mathfrak{h}\left(1, \mathbf{x}_{i}+\frac{1}{2} \bar{\gamma} ; 0\right)+\bar{\gamma}\left(1+x_{i}\right)+\frac{1}{4} \overline{\boldsymbol{\gamma}}^{2} \geqslant \mathbf{a}_{i}\right) \\
+ & \mathbb{P}\left(\mathfrak{h}\left(1, \mathbf{x}_{i}-\frac{1}{2} \bar{\gamma} ; 0\right)+\bar{\gamma}\left(1-x_{i}\right)+\frac{1}{4} \overline{\boldsymbol{\gamma}}^{2} \geqslant \mathbf{a}_{i}\right) \\
=2- & F_{\mathrm{GOE}}\left(4^{1 / 3}\left(\mathbf{a}_{i}-\bar{\gamma}\left(1+x_{i}\right)-\frac{1}{4} \overline{\boldsymbol{\gamma}}^{2}\right)\right) \\
& -F_{\mathrm{GOE}}\left(4^{1 / 3}\left(\mathbf{a}_{i}-\bar{\gamma}\left(1-x_{i}\right)-\frac{1}{4} \overline{\boldsymbol{\gamma}}^{2}\right)\right) \\
\gtrsim & e^{-(4 / 3)\left(\mathbf{t}^{-1 / 3} \min _{i} \mathbf{a}_{i}\right)^{3 / 2}},
\end{aligned}
$$

which is what we want. 
Remark 4.8. (Replicas and factorization ansatz) An earlier attempt [20] based on non-rigorous replica methods gave a formula which does not appear to be the same (though there is room for two apparently different Fredholm determinants to coincide). The replica derivation uses both divergent series and an asymptotic factorization assumption [47] for the Bethe eigenfunctions of the delta Bose gas. The divergent series are regularized through the Airy trick, which uses the identity

$$
\int d x \operatorname{Ai}(x) e^{n x}=e^{n^{3} / 3}
$$

to obtain

$$
\sum_{n=0}^{\infty}(-1)^{n} e^{n^{3} / 3} "=" \int d x \operatorname{Ai}(x) \sum_{n=0}^{\infty}(-1)^{n} e^{n x}=\int d x \operatorname{Ai}(x) \frac{1}{1+e^{x}}
$$

Although there is no justification, it is widely accepted in the field that the Airy trick gives consistently correct answers in KPZ. The factorization assumption, on the other hand, has only been justified by the fact that it has led to the correct result in a few previously known cases.

Remark 4.9. (Extension in time) TASEP has the unusual property that the initial value problem where we start with $h_{0}(z)$, and solve for the process $h_{t}(z), t>0$, can also be done backwards in time. This is just because the backwards-in-time dynamics is nothing but the forward-in-time dynamics for $-h$. So, we can immediately extend the process to $h_{t}(z),-\infty<t<\infty$. Of course, for some initial data, such as step $h_{0}(z)=|z|$, there will be no movement on $(-\infty, 0]$. The same property is inherited by the KPZ fixed point, except for the not technical point that, even if $-\mathfrak{h}_{0}$ were upper-semicontinuous, it might no longer lie in UC if it violates the linear growth condition. For example, $\mathfrak{h}_{0}(\mathbf{x})=-\kappa \mathbf{x}^{2}, \kappa>0$, is good initial data for the KPZ fixed point, but $-\mathfrak{h}_{0}$ has a finite lifetime $[0,1 / \kappa)$, after which it "explodes" to $+\infty$. So, the initial value problem for the fixed point on UC has an extension to $\left(\mathbf{t}_{0}, \infty\right)$, where $\mathbf{t}_{0} \leqslant 0$. The narrow wedge initial data is an example where $\mathbf{t}_{0}=0$. Continuous $\mathfrak{h}_{0}$, with $-\mathfrak{h}_{0}$ satisfying the linear growth condition, have $\mathbf{t}_{0}=-\infty$.

Remark 4.10. (Domain Markov property) (Suggested by M. Hairer) The KPZ fixed point inherits a stronger space-time Markov property from TASEP, which we describe informally and without complete proofs.

First, we state the domain Markov property of the space-time TASEP height function $h_{t}(z)$. It is clear from the definition of TASEP that, given the height function at $z \in \mathbb{Z}$ over some time interval $\left[t_{1}, t_{2}\right]$, what happens to the height function strictly to the right of $z$ over that time interval is independent of what happens strictly to the left. Bootstrapping 
from this, we see that if $A$ is any connected open subset of $(-\infty, \infty) \times \mathbb{Z}$ which is a finite union of rectangles $\left(\mathbf{t}_{1}, \mathbf{t}_{2}\right) \times\left(\mathbf{x}_{1}, \mathbf{x}_{2}\right)$ (some of which could be infinite), then $h_{t}(z)$ has the domain Markov property: If we call the boundary of a subset of the integers those at distance exactly 1 from the set, then $\left\{h_{t}(z):(t, z) \in A\right\}$ and $\left\{h_{t}(z):(t, z) \in(A \cup \partial A)^{\mathrm{c}}\right\}$ are independent, given $\left\{h_{t}(z):(t, z) \in \partial A\right\}$.

Now, let $\mathfrak{h}(\mathbf{t}, \mathbf{x})$ be the KPZ fixed point on $\left(\mathbf{t}_{0}, \infty\right) \times \mathbb{R}$, and let $A$ be a connected open subset of this domain with a regular boundary $\partial A$. Let

$$
\mathcal{G}_{\partial A}=\bigcap_{\substack{O \supset \partial A \\ O \text { open }}} \sigma\{\mathfrak{h}(\mathbf{t}, \mathbf{x}):(\mathbf{t}, \mathbf{x}) \in O\}
$$

be the germ field of the boundary. Taking limits from TASEP we see that

$$
\{\mathfrak{h}(\mathbf{t}, \mathbf{x}):(\mathbf{t}, \mathbf{x}) \in A\} \quad \text { and } \quad\left\{\mathfrak{h}(\mathbf{t}, \mathbf{x}):(\mathbf{t}, \mathbf{x}) \in(A \cup \partial A)^{\complement}\right\}
$$

are independent, given $\mathcal{G}_{\partial A}$. One expects that $\mathcal{G}_{\partial A}$ actually equals

$$
\sigma(\{\mathfrak{h}(\mathbf{t}, \mathbf{x}):(\mathbf{t}, \mathbf{x}) \in \partial A\})
$$

but it is not immediately clear how to prove this.

An important consequence is that the fixed point is not just a Markov process in $\mathbf{t}$, it is also a Markov process sideways in $\mathbf{x}$. This may partially explain results like the recent 2-time formulas [4], [35]-[37]. At any rate, it means that, while our description of the KPZ fixed point as a Markov process in $\mathbf{t}$ is the first characterization of the field, it is far from a complete description (see also Remark 4.23).

Remark 4.11. (Locality) There are various notions of locality, the domain Markov property above being one; an even stronger statement of locality would follow if we knew the sharp version $\mathcal{G}_{\partial A}=\sigma(\{\mathfrak{h}(\mathbf{t}, \mathbf{x}):(\mathbf{t}, \mathbf{x}) \in \partial A\})$.

More concretely, one could ask whether

$$
\mid \mathbb{P}_{\mathfrak{h}_{0}}\left(\mathfrak{h}\left(\mathbf{t}, \mathbf{x}_{i}\right) \leqslant \mathbf{a}_{i} \text { for } i=1, \ldots, M\right)-\mathbb{P}_{\mathfrak{h}_{0}^{\delta}}\left(\mathfrak{h}\left(\mathbf{t}, \mathbf{x}_{i}\right) \leqslant \mathbf{a}_{i} \text { for } i=1, \ldots, M\right) \mid=o(\mathbf{t})
$$

as $\mathbf{t} \rightarrow 0$, whenever $\mathfrak{h}_{0}^{\delta} \in \mathrm{UC}$ is such that $\mathfrak{h}_{0}^{\delta}(\mathbf{y})=\mathfrak{h}_{0}(\mathbf{y})$ if $\left|\mathbf{y}-\mathbf{x}_{i}\right|<\delta$ for each $i$. From the variational formula (4.18), it is fairly straightforward to bound the left-hand side of (4.11) by $\exp \left\{-C \delta^{3} / \mathbf{t}^{2}\right\}$ providing a strong statement of locality. Presumably, this could differentiate between the true fixed point and the non-local stochastic partial differential equation suggested in footnote 13 . The functions have to be in UC; if they are allowed to grow quadratically, then there are counterexamples. 
Remark 4.12. (Uniqueness and strong KPZ universality conjectures) The KPZ fixed point is expected to be the unique non-trivial (i.e. non-zero) space-time field satisfying locality in the sense of (4.11) and Theorem 4.5 (i), (iii) and (iv) (the inviscid limit given by (1.3) satisfies all but (iii)).

The strong KPZ universality conjecture states that the KPZ fixed point is the limit under the 1:2:3 scaling of all models in the KPZ universality class. This last statement can alternately be interpreted as the definition of the universality class. Note that it appears to exclude models such as vicious walkers and random matrices, which have KPZ-type fluctuations, but seem to lack a meaningful analogue of a large class of initial conditions.

From Theorem 3.9, we obtain the following result.

TheOrem 4.13. (Hölder $\frac{1}{2}$ - regularity in space) Fix $\mathbf{t}>0, \mathfrak{h}_{0} \in \mathrm{UC}$, and let $\mathfrak{h}(\mathbf{t})$ denote the fixed point at time $\mathbf{t}$. Then, for each $\beta \in\left(0, \frac{1}{2}\right)$ and $M<\infty$, we have

$$
\lim _{A \rightarrow \infty} \mathbb{P}\left(\|\mathfrak{h}(\mathbf{t})\|_{\beta,[-M, M]} \geqslant A\right)=0 .
$$

The bounds on the trace norms used to prove Theorem 4.13 also yield the local Brownian property for the fixed point (the proof is exactly the same as [48], with $\mathbf{K}_{\mathbf{t}}^{\text {hypo }\left(\mathfrak{h}_{0}\right)}$ replacing $B_{0}$ there).

Theorem 4.14. (Local Brownian behavior) For any $\mathbf{t}>0$ and any initial condition $\mathfrak{h}_{0} \in \mathrm{UC}, \mathfrak{h}(\mathbf{t}, \mathbf{x})$ is locally Brownian in $\mathbf{x}$, in the sense $\left({ }^{25}\right)$ that, for each $\mathbf{y} \in \mathbb{R}$, the finite-dimensional distributions of $\mathfrak{b}_{\varepsilon}(\mathbf{x})=\varepsilon^{-1 / 2}(\mathfrak{h}(\mathbf{t}, \mathbf{y}+\varepsilon \mathbf{x})-\mathfrak{h}(\mathbf{t}, \mathbf{y}))$ converge, as $\varepsilon \searrow 0$, to those of a double-sided Brownian motion $\mathbf{B}$ with diffusion coefficient 2 and $\mathbf{B}(0)=0$.

By the 1:2:3 scaling invariance, Theorem $4.5(\mathrm{i})$, we have

$$
\mathfrak{h}\left(\mathbf{t}, \mathbf{x} ; \mathfrak{h}_{0}\right) \stackrel{\text { dist }}{=} \mathbf{t}^{1 / 3} \mathfrak{h}\left(1, \mathbf{t}^{-2 / 3} \mathbf{x} ; \mathbf{t}^{-1 / 3} \mathfrak{h}_{0}\left(\mathbf{t}^{2 / 3} \mathbf{x}\right)\right)
$$

Hence, the local Brownian behaviour of the fixed point is essentially equivalent to ergodicity. Recall (see the comment after Theorem 4.5) that, for any $\rho \in \mathbb{R}$, drifted Brownian motion $\mathbf{B}(\mathbf{x})+\rho \mathbf{x}$ is invariant for the fixed point. The following gives a fairly general condition on initial data in UC to see $\mathbf{B}(\mathbf{x})+\rho \mathbf{x}$ locally after a long time.

Theorem 4.15. (Ergodicity) For any (possibly random) initial condition $\mathfrak{h}_{0} \in \mathrm{UC}$ such that, for some $\rho \in \mathbb{R}$,

$$
\varepsilon^{1 / 2}\left(\mathfrak{h}_{0}\left(\varepsilon^{-1} \mathbf{x}\right)-\rho \varepsilon^{-1} \mathbf{x}\right)
$$

$\left({ }^{25}\right)$ Since the first version of this article was posted, there has been progress on the stronger statement of absolute continuity with respect to Brownian motion on finite intervals [15], [31], [53]. This uses a different class of approximating models which are shown to converge to the fixed point in [43]. 
is convergent, in distribution, in $\mathrm{UC}$, the finite-dimensional distributions of the process

$$
\mathfrak{h}\left(\mathbf{t}, \mathbf{x} ; \mathfrak{h}_{0}\right)-\mathfrak{h}\left(\mathbf{t}, 0 ; \mathfrak{h}_{0}\right)-\rho \mathbf{x}
$$

converge, as $\mathbf{t} \rightarrow \infty$, to those of a double-sided Brownian motion $\mathbf{B}$ with diffusion coefficient 2 .

A similar result was first proved by Pimentel [44] using coupling, in an article which appeared after the first version of this paper was posted. The present theorem was added in the second version.

Proof. By the 1:2:3 scaling and affine invariance properties, Theorem 4.5 (i) and (vi), (4.13) is equal in distribution to

$$
\mathbf{t}^{1 / 3}\left(\mathfrak{h}\left(1, \mathbf{t}^{-2 / 3} \mathbf{x} ; \mathbf{t}^{-1 / 3}\left(\mathfrak{h}_{0}\left(\mathbf{t}^{2 / 3} \mathbf{x}\right)-\rho \mathbf{t}^{2 / 3} \mathbf{x}\right)\right)-\mathfrak{h}\left(1,0 ; \mathbf{t}^{-1 / 3}\left(\mathfrak{h}_{0}\left(\mathbf{t}^{2 / 3} \mathbf{x}\right)-\rho \mathbf{t}^{2 / 3} \mathbf{x}\right)\right)\right)
$$

Since the initial condition converges in UC, one can repeat the proof of local Brownian behaviour from [48], using now the fact that, if $\mathfrak{h}^{\varepsilon} \rightarrow \mathfrak{h}$ in $\mathrm{UC}$, then $\left.\mathbf{K}_{\mathbf{t}}^{\text {hypo(h⿱一一) }} \rightarrow^{\varepsilon}\right) \mathbf{K}_{\mathbf{t}}^{\text {hypo(h) }}$ in trace norm.

\subsection{Recovery of the Airy processes}

Although the determinantal formula (4.7) used in the definition of the KPZ fixed point looks imposing, we easily recover several of the classical Airy processes $\left({ }^{26}\right)$ by starting with special initial data for which the hitting times are explicit, and observing the spatial process at time $\mathbf{t}=1$.

Start by considering the UC function $\mathfrak{d}_{\mathbf{u}}(\mathbf{u})=0, \mathfrak{d}_{\mathbf{u}}(\mathbf{x})=-\infty$ for $\mathbf{x} \neq \mathbf{u}$, known as a narrow wedge at $\mathbf{u}$. It leads to the Airy 2 process (sometimes simply the Airy process):

$$
\mathfrak{h}\left(1, \mathbf{x} ; \mathfrak{d}_{\mathbf{u}}\right)+(\mathbf{x}-\mathbf{u})^{2}=\mathcal{A}_{2}(\mathbf{x}) \quad(\text { sometimes simply } \mathcal{A}(\mathbf{x}))
$$

Flat initial data $\mathfrak{h}_{0} \equiv 0$, on the other hand, leads to the Airy 1 process:

$$
\mathfrak{h}(1, \mathbf{x} ; 0)=2^{1 / 3} \mathcal{A}_{1}\left(2^{-2 / 3} \mathbf{x}\right)
$$

$\left({ }^{26}\right)$ Besides the ones we treat here, there are three more basic Airy processes $\mathcal{A}_{\text {stat }}, \mathcal{A}_{1 \rightarrow \mathrm{BM}}$ and $\mathcal{A}_{2 \rightarrow \mathrm{BM}}$, obtained respectively by starting from a 2 -sided Brownian motion, a 1-sided Brownian motion to the right of the origin and zero to the left of the origin, and a 1-sided Brownian motion to the right of the origin and $-\infty$ to the left of the origin [3], [12], [16], [32]. However, using (4.7), in these cases involves averaging over the initial randomness, and hence verifying directly that the resulting formulas coincide with those in the literature is more challenging. 
Finally, the UC function $\mathfrak{h}_{\mathrm{h}-\mathrm{f}}(\mathbf{x})=-\infty$ for $\mathbf{x}<0, \mathfrak{h}_{\mathrm{h}-\mathrm{f}}(\mathbf{x})=0$ for $\mathbf{x} \geqslant 0$, called wedge or half-flat initial data, leads to the Airy $_{2 \rightarrow 1}$ process:

$$
\mathfrak{h}\left(1, \mathbf{x} ; \mathfrak{h}_{\mathrm{h}-\mathrm{f}}\right)+\mathbf{x}^{2} \mathbf{1}_{\mathbf{x}<0}=\mathcal{A}_{2 \rightarrow 1}(\mathbf{x})
$$

Formulas for the $m$-point distributions of these special solutions were obtained in the 2000s in [9]-[11], [34], [45], [54], [55], in terms of Fredholm determinants of extended kernels, and later in terms of path-integral kernels in [8], [19], [48]. The Airy ${ }_{2 \rightarrow 1}$ process interpolates between the other two in the limits $\mathbf{x} \rightarrow-\infty$ and $\mathbf{x} \rightarrow \infty$.

We now show how the formula for the Airy $_{2 \rightarrow 1}$ process arises from the KPZ fixed point formula (4.7). The Airy 1 and Airy 2 processes can be obtained analogously (or in the limits $\mathbf{x} \rightarrow \pm \infty)$. We have to take

$$
\mathfrak{h}_{0}(\mathbf{x})= \begin{cases}-\infty, & \text { for } \mathbf{x}<0 \\ 0, & \text { for } \mathbf{x} \geqslant 0\end{cases}
$$

in (4.7). It is straightforward to check that $\mathbf{S}_{\mathbf{t}, 0}^{\text {hypo }\left(\mathfrak{h}_{0}^{-}\right)}=\bar{\chi}_{0} \mathbf{S}_{\mathbf{t}, 0}$, so that

$$
\left(\mathbf{S}_{\mathbf{t}, 0}-\mathbf{S}_{\mathbf{t}, 0}^{\mathrm{hypo}\left(\mathfrak{h}_{0}^{-}\right)}\right)^{*}=\left(\mathbf{S}_{\mathbf{t}, 0}\right)^{*} \chi_{0} .
$$

On the other hand, an application of the reflection principle based on (3.12) (see [51, Proposition 3.6] for the details in the case $\mathbf{t}=1$ ) yields that, for $v \geqslant 0$ (using (3.14) and writing $\boldsymbol{\tau}_{0}$ for the hitting time of zero by $\mathbf{B}$ ),

$$
\mathbf{S}_{\mathbf{t}, 0}^{\operatorname{hypo}\left(\mathfrak{h}_{0}^{+}\right)}(v, u)=\mathbf{S}_{-\mathbf{t}, 0}^{\text {epi }\left(-\mathfrak{h}_{0}^{+}\right)}(-v,-u)=\int_{0}^{\infty} \mathbb{P}_{-v}\left(\boldsymbol{\tau}_{0} \in d \mathbf{y}\right) \mathbf{S}_{-\mathbf{t},-\mathbf{y}}(0,-u)=\mathbf{S}_{\mathbf{t}, 0}(-v, u),
$$

which gives

$$
\mathbf{K}_{\mathbf{t}}^{\mathrm{hypo}\left(\mathfrak{h}_{0}\right)}=\mathbf{I}-\left(\mathbf{S}_{\mathbf{t}, 0}\right)^{*} \chi_{0}\left[\mathbf{S}_{\mathbf{t}, 0}-\varrho \mathbf{S}_{\mathbf{t}, 0}\right]=\left(\mathbf{S}_{\mathbf{t}, 0}\right)^{*}(\mathbf{I}+\varrho) \bar{\chi}_{0} \mathbf{S}_{\mathbf{t}, 0},
$$

where $\varrho$ is the reflection operator $\varrho f(x)=f(-x)$. Hence,

$$
\mathbf{K}_{\mathbf{t}, \operatorname{ext}}^{\mathrm{hypo}\left(\mathfrak{h}_{0}\right)}\left(\mathbf{x}_{i}, \cdot ; \mathbf{x}_{j}, \cdot\right)=-e^{\left(\mathbf{x}_{j}-\mathbf{x}_{i}\right) \partial^{2}} \mathbf{1}_{\mathbf{x}_{i}<\mathbf{x}_{j}}+\left(\mathbf{S}_{\mathbf{t},-\mathbf{x}_{i}}\right)^{*}(\mathbf{I}+\varrho) \bar{\chi}_{0} \mathbf{S}_{\mathbf{t}, \mathbf{x}_{j}} .
$$

Setting $\mathbf{t}=1$, we get

$$
\mathbf{K}_{1, \text { ext }}^{\text {hypo }\left(\mathfrak{h}_{0}\right)}\left(\mathbf{x}_{i}, u_{i}-\mathbf{x}_{i}^{2} \mathbf{1}_{\mathbf{x}_{i} \leqslant 0} ; \mathbf{x}_{j}, u_{j}-\mathbf{x}_{j}^{2} \mathbf{1}_{\mathbf{x}_{j} \leqslant 0}\right)=K_{2 \rightarrow 1}\left(\mathbf{x}_{i}, u_{i} ; \mathbf{x}_{j}, u_{j}\right)
$$

where $K_{2 \rightarrow 1}$ is the extended kernel for the Airy ${ }_{2 \rightarrow 1}$ process, as given in [49, equation 1.8] (see also [11]). Therefore,

$$
\mathbb{P}\left(\mathfrak{h}\left(1, \mathbf{x}_{i} ; \mathfrak{h}_{0}\right) \leqslant \mathbf{a}_{i} \text { for } i=1, \ldots, m\right)=\mathbb{P}\left(\mathcal{A}_{2 \rightarrow 1}\left(\mathbf{x}_{i}\right)-\mathbf{x}_{i}^{2} \mathbf{1}_{\mathbf{x}_{i} \leqslant 0} \leqslant \mathbf{a}_{i} \text { for } i=1, \ldots, m\right)
$$


Remark 4.16. Theorem 3.8 gives a much stronger statement about universality of the Airy processes with respect to initial conditions than was previously known (for 1-point marginals this appears in [17], and to some extent [51]): if we start with two rescaled TASEP height functions $\mathfrak{h}_{0}^{\varepsilon, 1}$ and $\mathfrak{h}_{0}^{\varepsilon, 2}$ which converge in distribution in UC to the same limit $\mathfrak{h}_{0}$ as $\varepsilon \rightarrow 0$, then, for any $\mathbf{t}>0, \mathfrak{h}^{\varepsilon, 1}(\mathbf{t}, \cdot)$ and $\mathfrak{h}^{\varepsilon, 2}(\mathbf{t}, \cdot)$ have the same (distributional) limit.

For example, for some fixed $\kappa>0$, one could consider a TASEP initial condition obtained from the periodic case $X_{0}(i)=2 i, i \in \mathbb{Z}$, by taking, for each $j \geqslant 1$, the particle at the position $\bar{j}$ which is closest to $2|j|^{1 / \kappa}$, and moving it to $-\bar{j}-1$, which leads to an initial TASEP height function $h_{0}(i) \approx-|i|^{\kappa}$. If $\kappa<\frac{1}{2}$, then $\mathfrak{h}^{\varepsilon}(0, \cdot) \rightarrow 0$ as $\varepsilon \rightarrow 0$, and thus $\mathfrak{h}^{\varepsilon}(1, \cdot)$ converges to the Airy 1 process, while if $\kappa>\frac{1}{2}$, then $\mathfrak{h}^{\varepsilon}(0, \cdot) \rightarrow \mathfrak{d}_{0}$ and $\mathfrak{h}^{\varepsilon}(1, \cdot)$ converges to the Airy $_{2}$ process. A statement like this appears to have been outside of the scope of previous arguments.

\subsection{Variational formulas}

The KPZ fixed point satisfies a version (see (4.18) below) of the Hopf-Lax variational formula (1.3) with a new noise.

Example 4.17. (Airy sheet) The 2-parameter process

$$
\mathcal{A}(\mathbf{x}, \mathbf{y})=\mathfrak{h}\left(1, \mathbf{y} ; \mathfrak{d}_{\mathbf{x}}\right)+(\mathbf{x}-\mathbf{y})^{2}
$$

is called the Airy sheet (here, $\mathfrak{d}_{\mathbf{x}}$ is the narrow wedge defined in the last section). In some contexts it is better to include the parabola, so one writes

$$
\hat{\mathcal{A}}(\mathbf{x}, \mathbf{y})=\mathcal{A}(\mathbf{x}, \mathbf{y})-(\mathbf{x}-\mathbf{y})^{2}
$$

Several remarks are in order:

- The KPZ fixed point formula does not give explicit joint probabilities

$$
\mathbb{P}\left(\mathcal{A}\left(\mathbf{x}_{i}, \mathbf{y}_{i}\right) \leqslant \mathbf{a}_{i} \text { for } i=1, \ldots, m\right)
$$

for the Airy sheet, $\left({ }^{27}\right)$ and we presently have no method to obtain them.

$\left({ }^{27}\right)$ The most general formula we can get from the results in $\S 4.2$ comes from Theorem 4.4 and reads

$$
\mathbb{P}(\hat{\mathcal{A}}(\mathbf{x}, \mathbf{y}) \leqslant \mathfrak{f}(\mathbf{x})+\mathfrak{g}(\mathbf{y}) \text { for } \mathbf{x}, \mathbf{y} \in \mathbb{R})=\operatorname{det}\left(\mathbf{I}-\mathbf{K}_{1 / 2}^{\text {hypo }(-\mathfrak{g})} \mathbf{K}_{-1 / 2}^{\text {epi }(\mathfrak{f})}\right) .
$$

Even in the case when $\mathfrak{f}$ and $\mathfrak{g}$ take two non-infinite values, it gives a formula for

$$
\mathbb{P}\left(\hat{\mathcal{A}}\left(\mathbf{x}_{i}, \mathbf{y}_{j}\right) \leqslant \mathfrak{f}\left(\mathbf{x}_{i}\right)+\mathfrak{g}\left(\mathbf{y}_{j}\right) \text { for } i, j=1,2\right),
$$

but $\mathfrak{f}\left(\mathbf{x}_{i}\right)+\mathfrak{g}\left(\mathbf{y}_{j}\right)$ only span a 3-dimensional linear subspace of $\mathbb{R}^{4}$. So, it does not determine the joint distribution of $\hat{\mathcal{A}}\left(\mathbf{x}_{i}, \mathbf{y}_{j}\right), i, j=1,2$. 
- Existence of the Airy sheet is obtained in our context from subsequential limits; see Remark 4.21. While our methods leave open the question of uniqueness, this has been proved since the present article was submitted in [23]; see Remark 4.23. Since they start from a different model, one needs to combine their result with [43].

- By stationarity in space, Theorem 4.5 (iv),

$$
\mathfrak{h}\left(1, \mathbf{y} ; \mathfrak{d}_{\mathbf{x}}\right) \stackrel{\text { dist }}{=} \mathfrak{h}\left(1, \mathbf{y}-\mathbf{x} ; \mathfrak{d}_{0}\right),
$$

and by reflection invariance, Theorem $4.5(\mathrm{v})$, since $\mathfrak{d}_{0}(-\mathbf{x})=\mathfrak{d}_{0}(\mathbf{x})$ we have

$$
\mathfrak{h}\left(1, \mathbf{y}-\mathbf{x} ; \mathfrak{d}_{0}\right) \stackrel{\text { dist }}{=} \mathfrak{h}\left(1, \mathbf{x}-\mathbf{y} ; \mathfrak{d}_{0}\right) .
$$

This gives the permutation symmetry

$$
\mathcal{A}(\mathbf{x}, \mathbf{y}) \stackrel{\text { dist }}{=} \mathcal{A}(\mathbf{y}, \mathbf{x}) \text {. }
$$

- Fixing either variable $\mathbf{x}$ or $\mathbf{y}, \mathcal{A}(\mathbf{x}, \mathbf{y})$ is an Airy 2 process in the other.

- The Airy sheet is stationary: $\left({ }^{28}\right)$ for any fixed $\mathbf{x}_{0}, \mathbf{y}_{0}$,

$$
\mathcal{A}\left(\mathbf{x}+\mathbf{x}_{0}, \mathbf{y}+\mathbf{y}_{0}\right) \stackrel{\text { dist }}{=} \mathcal{A}(\mathbf{x}, \mathbf{y}) .
$$

- New non-obvious distributional symmetries of the Airy sheet have been discovered and conjectured recently (see $[13, \S 1.5$ and $\S 1.6]$ ).

By repeated application of Theorem 4.5 (vii) to initial data which take finite values $\mathfrak{h}_{0}\left(\mathbf{x}_{i}\right)$ at $\mathbf{x}_{i}, i=1, \ldots, n$, and $-\infty$ everywhere else), and then taking limits, we obtain the following result.

Theorem 4.18. (Airy sheet variational formula) For each $\mathbf{t}>0$,

$$
\mathfrak{h}\left(\mathbf{t}, \mathbf{x} ; \mathfrak{h}_{0}\right) \stackrel{\text { dist }}{=} \sup _{\mathbf{y} \in \mathbb{R}}\left\{\mathbf{t}^{1 / 3} \mathcal{A}\left(\mathbf{t}^{-2 / 3} \mathbf{x}, \mathbf{t}^{-2 / 3} \mathbf{y}\right)-\frac{1}{\mathbf{t}}(\mathbf{x}-\mathbf{y})^{2}+\mathfrak{h}_{0}(\mathbf{y})\right\}
$$

as processes in $\mathbf{x}$. In particular, $\mathcal{A}$ satisfies the semi-group property: if $\hat{\mathcal{A}}^{1}$ and $\hat{\mathcal{A}}^{2}$ are independent copies (with parabolas included) and $\mathbf{t}_{1}+\mathbf{t}_{2}=\mathbf{t}$ are all positive, then

$$
\sup _{\mathbf{z}}\left\{\mathbf{t}_{1}^{1 / 3} \hat{\mathcal{A}}^{1}\left(\mathbf{t}_{1}^{-2 / 3} \mathbf{x}, \mathbf{t}_{1}^{-2 / 3} \mathbf{z}\right)+\mathbf{t}_{2}^{1 / 3} \hat{\mathcal{A}}^{2}\left(\mathbf{t}_{2}^{-2 / 3} \mathbf{z}, \mathbf{t}_{2}^{-2 / 3} \mathbf{y}\right)\right\} \stackrel{\text { dist }}{=} \mathbf{t}^{1 / 3} \hat{\mathcal{A}}^{1}\left(\mathbf{t}^{-2 / 3} \mathbf{x}, \mathbf{t}^{-2 / 3} \mathbf{y}\right) .
$$

$\left({ }^{28}\right)$ Using the methods of this paper one can very easily prove (4.17) in the case $\mathbf{x}_{0}=\mathbf{y}_{0}$; a proof of the general statement can be found in [23] (see Remark 4.23). 
Remark 4.19. The equalities in distribution (4.18) and (4.19) hold only for fixed $\mathbf{t}_{1}, \mathbf{t}_{2}$ and $\mathbf{t}$, and not as processes in $\mathbf{t}$. If $\mathbf{t}^{1 / 3} \mathcal{A}\left(\mathbf{t}^{-2 / 3} \mathbf{x}, \mathbf{t}^{-2 / 3} \mathbf{y}\right)$ on the right-hand side of (4.18) is replaced by $\mathbf{t}^{1 / 3} \mathcal{A}_{2}\left(\mathbf{t}^{-2 / 3}(\mathbf{x}-\mathbf{y})\right)$, the equality in distribution holds for each fixed $\mathbf{x}$ and $\mathbf{t}$, but no longer as processes in $\mathbf{x}$.

Example 4.20. From (4.15) and (4.18), we deduce that the Airy 1 process satisfies

$$
2^{1 / 3} \mathcal{A}_{1}\left(2^{-2 / 3} \mathbf{x}\right) \stackrel{\text { dist }}{=} \sup _{\mathbf{y} \in \mathbb{R}}\left\{\mathcal{A}(\mathbf{x}, \mathbf{y})-(\mathbf{x}-\mathbf{y})^{2}\right\}
$$

generalizing the famous identity of Johansson [34] that the GOE Tracy-Widom distribution can be written as the sup of the Airy 2 process minus a parabola. The odd factors of $2^{1 / 3}$ on the left-hand side are the result of a mismatch in natural normalization between the original interpretation from random matrices, and the present one from growth models.

Remark 4.21. (Existence of Airy sheets) In TASEP there is a canonical coupling between the process starting from different initial conditions. Take independent Poisson processes of rate 1 , one for each site $x$. When the Poisson process at $x$ jumps, the TASEP height function jumps down by 2 if and only if $h(x)$ is a local maximum. The coupling just means to use the same background Poisson processes for several different evolving height functions. It is clear that, under such a coupling, the TASEP version of the preservation of max property (Theorem 4.5 (vii)) holds. This seems to have been first exploited by [57], and leads to the result for the KPZ fixed point in the 1:2:3 limit.

Indeed, let $\mathcal{A}^{\varepsilon}(\mathbf{x}, \mathbf{y})$ denote the 1:2:3 rescaled and recentered (as in (3.1)) TASEP version of the Airy sheet: $\mathcal{A}^{\mathrm{TASEP}}(x, y)=h(1, y ;-|\cdot-x|)$, i.e. the TASEP height function at $y$ at time 1 starting with packed particles to the left of $x$. Let $p_{\left(\mathbf{x}_{1}, \mathbf{y}_{1}\right), \ldots,\left(\mathbf{x}_{n}, \mathbf{y}_{n}\right)}^{\varepsilon}$ denote the joint distribution of $\mathcal{A}^{\varepsilon}\left(\mathbf{x}_{1}, \mathbf{y}_{1}\right), \ldots, \mathcal{A}^{\varepsilon}\left(\mathbf{x}_{n}, \mathbf{y}_{n}\right)$. These are a consistent family of finitedimensional distributions, and the corresponding distributions $\mathbb{P}^{\varepsilon}$ of the approximating Airy sheets $\mathcal{A}^{\varepsilon}(\mathbf{x}, \mathbf{y})$ are tight in $\mathscr{C}\left(\mathbb{R}^{2}\right)$, since they satisfy the Hölder bounds (3.28) uniformly in $\varepsilon$, in each variable separately, from the permutation symmetry (which holds at the TASEP level), and therefore in both variables, since the Hölder norm of a function of two variables is easily controlled by the sum of the Hölder norms in each variable. Any limiting process is called an Airy sheet, and clearly satisfies (4.16)-(4.19).( $\left.{ }^{29}\right)$

Either through (4.18), or using a similar construction to the previous paragraph, one produces a basic coupling of the KPZ fixed point starting with different initial data. So the KPZ fixed point can be thought of as a stochastic flow.

$\left({ }^{29}\right)$ It is interesting that although we are unable to prove uniqueness, the variational formulas (4.18) and (4.19) hold for any such limit, especially since the left-hand side of (4.18) is unique. 
Example 4.22. As a direct consequence of the variational formulas we deduce that if $\mathfrak{h}_{\varepsilon}(0, \cdot) \rightarrow \mathfrak{h}_{0}$ in distribution in UC, then the asymptotic fluctuations of the rescaled TASEP height function $\mathfrak{h}_{\varepsilon}(1, \mathbf{x})$ at a single point $\mathbf{x}$ (and time $\mathbf{t}=1$ ) have the same distribution as $\sup _{\mathbf{y} \in \mathbb{R}}\left\{\mathcal{A}_{2}(\mathbf{x}-\mathbf{y})-(\mathbf{x}-\mathbf{y})^{2}+\mathfrak{h}_{0}(\mathbf{y})\right\}$. This is the result proved in [17] in the context of discrete time TASEP with sequential update (although their assumptions on the convergence of the initial data are different). It also coincides, in essence, $\left({ }^{30}\right)$ with the result proved in [51] about the 1-point fluctuations for the KPZ equation with general initial data, providing further evidence for the strong KPZ universality conjecture.

Remark 4.23. The directed landscape was recently constructed in [23] as a (nonexplicit) functional of the Airy line ensemble. This process was earlier called the spacetime Airy sheet in [20]. This nails down the right-hand side of the variational formula (4.18), or, alternatively, one can think of the variational formula as defining the KPZ fixed point in terms of the Airy sheet. Such a definition can be useful to obtain qualitative properties of the fixed point, though, as we see in this section, many of them do not rely on uniqueness of the Airy sheet, but only its local, Brownian behaviour. Qualitative properties of the sheet/landscape are obtained in [23], but the key point of the present article, the integrability of the fixed point, does not (at the present time) appear to extend to the sheet.

\subsection{Regularity in time}

We have seen that the fixed point is locally Hölder $\frac{1}{2}$ - regular in space, and thus from the 1:2:3 scaling variance (Theorem 4.5 (i)) one expects that it is also locally Hölder $\frac{1}{3}-$ regular in time. This can be proved as an application of the variational formula (4.18); in fact, for this purpose, one only needs the pointwise, and not process level, version of the variational formula, so on the right-hand side we can replace the Airy sheet by an Airy process (see Remark 4.19). To see this, fix $0<\mathbf{s}<\mathbf{t}, \mathbf{x}_{0} \in \mathbb{R}$, and $\alpha<\frac{1}{3}$, and choose $\beta<\frac{1}{2}$ such that $\beta /(2-\beta)=\alpha$. We want to compare $\mathfrak{h}\left(\mathbf{t}, \mathbf{x}_{0}\right)$ and $\mathfrak{h}_{0}\left(\mathbf{s}, \mathbf{x}_{0}\right)$, but from the Markov property and the fact that at time $\mathbf{s}$ the process is in $\mathscr{C}^{\beta}$, we may assume without loss of generality that $\mathbf{s}=0$ and $\mathfrak{h}_{0} \in \mathscr{C}^{\beta}$. There is an $R<\infty$ a.s. such that

$$
|\mathcal{A}(\mathbf{x})| \leqslant R\left(1+|\mathbf{x}|^{\beta}\right) \quad \text { and } \quad\left|\mathfrak{h}_{0}(\mathbf{x})-\mathfrak{h}_{0}\left(\mathbf{x}_{0}\right)\right| \leqslant R\left(\left|\mathbf{x}-\mathbf{x}_{0}\right|^{\beta}+\left|\mathbf{x}-\mathbf{x}_{0}\right|\right)
$$

$\left({ }^{30}\right)$ The precise connection with the result in [51] rests on an assumption which is widely believed to hold, but which currently escapes rigorous treatment (namely that the partially asymmetric exclusion process with step initial data converges to the Airy 2 process); see Theorem 1.5 in that paper and the discussion preceding it for more details. 
From the variational formula (4.18), $\left|\mathfrak{h}\left(\mathbf{t}, \mathbf{x}_{0}\right)-\mathfrak{h}\left(0, \mathbf{x}_{0}\right)\right|$ is then bounded by

$$
\sup _{\mathbf{x} \in \mathbb{R}}\left(R\left(\left|\mathbf{x}-\mathbf{x}_{0}\right|^{\beta}+\left|\mathbf{x}-\mathbf{x}_{0}\right|+\mathbf{t}^{1 / 3}+\mathbf{t}^{(1-2 \beta) / 3}|\mathbf{x}|^{\beta}\right)-\frac{1}{\mathbf{t}}\left(\mathbf{x}_{0}-\mathbf{x}\right)^{2}\right) \leqslant \widetilde{R} \mathbf{t}^{\beta /(2-\beta)} .
$$

In view of our choice of $\beta$, this yields the desired result.

Proposition 4.24. (Hölder $\frac{1}{3}$ - regularity in time) For any $0<\alpha<\frac{1}{3}$ and any $\mathbf{x}_{0} \in \mathbb{R}$, $\mathfrak{h}\left(\mathbf{t}, \mathbf{x}_{0}\right)$ is locally Hölder $\alpha$-regular in $\mathbf{t}>0$.

Remark 4.25. One does not really expect Proposition 4.24 to be true at $\mathbf{t}=0$, unless one starts with Hölder $\frac{1}{2}$ - regular initial data, because of the lateral growth mechanism. For example, we can take $\mathfrak{h}_{0}(\mathbf{x})=\mathbf{x}^{\beta} \mathbf{1}_{\mathbf{x}>0}$ with $\beta \in\left(0, \frac{1}{2}\right)$ and check using the variational formula that $\mathfrak{h}(\mathbf{t}, 0)-\mathfrak{h}(0,0) \sim \mathbf{t}^{\beta /(2-\beta)}$ for small $\mathbf{t}>0$, which can be much worse than Hölder $\frac{1}{3}$ - regularity. On the other hand, the narrow wedge solution does satisfy

$$
\mathfrak{h}\left(\mathbf{t}, 0 ; \mathfrak{d}_{0}\right)-\mathfrak{h}\left(0,0 ; \mathfrak{d}_{0}\right) \sim \mathbf{t}^{1 / 3}
$$

At other points, $\mathfrak{h}\left(0, \mathbf{x} ; \mathfrak{d}_{0}\right)=-\infty$, while $\mathfrak{h}\left(\mathbf{t}, \mathbf{x} ; \mathfrak{d}_{0}\right)>-\infty$, so there is not much sense to time continuity at a point. It should be measured instead in UC, which we leave for future work.

\subsection{Equilibrium space-time covariance}

White noise plus an arbitrary height shift $\rho \in \mathbb{R}$ is invariant for the distribution-valued spatial derivative process $\mathfrak{u}=\partial_{x} \mathfrak{h}$ (see the remarks after Theorem 4.5) which could be called the stochastic Burgers fixed point, since it is expected to be the 1:2:3 scaling limit of the stochastic Burgers equation (introduced by [14])

$$
\partial_{t} u=\nu \partial_{x} u^{2}+\lambda \partial_{x}^{2} u+\sigma \partial_{x} \xi
$$

satisfied by $u=\partial_{x} h$ from (1.2). Dynamic renormalization was performed by [26] leading to the dynamic scaling exponent $\frac{3}{2}$. The equilibrium space-time covariance function was computed in [25] by taking a limit from TASEP: with $\lambda=\nu=\frac{1}{4}$ and $\sigma=1$, and setting $\rho=\frac{1}{2}$, one has

$$
\mathbb{E}[\mathfrak{u}(\mathbf{t}, \mathbf{x}) \mathfrak{u}(0,0)]=\frac{1}{2} \mathbf{t}^{-2 / 3} g_{\mathrm{sc}}^{\prime \prime}\left(\mathbf{t}^{-2 / 3} \mathbf{x}\right),
$$

where $g_{\mathrm{sc}}(w)=\int s^{2} d F_{w}(s)$, with $F_{w}(s)=\partial_{s}\left(F_{\mathrm{GUE}}\left(s+w^{2}\right) g\left(s+w^{2}, w\right)\right)$, and where

$$
g(s, w)=e^{-w^{3} / 3}\left[\int_{\mathbb{R}_{-}^{2}} d x d y e^{w(x+y)} \operatorname{Ai}(x+y+s)+\left\langle\hat{\Phi}_{w, s},\left(I-K_{\mathrm{Ai}, s}\right)^{-1} \hat{\Psi}_{w, s}\right\rangle_{L^{2}\left(\mathbb{R}_{+}\right)}\right],
$$


with

$$
\begin{aligned}
\hat{\Phi}_{w, s}(x) & =\int_{\mathbb{R}_{+}} d z e^{w z} K_{\mathrm{Ai}, s}(z, x) e^{w s}, \\
\hat{\Psi}_{w, s}(y) & =\int_{\mathbb{R}_{-}} d z e^{w z} \operatorname{Ai}(y+z+s), \\
K_{\mathrm{Ai}, s}(x, y) & =\int_{\mathbb{R}_{+}} d \lambda \operatorname{Ai}(\lambda+x+s) \operatorname{Ai}(\lambda+y+s) .
\end{aligned}
$$

Since $\mathfrak{u}(\mathbf{t}, \mathbf{x})$ is essentially a white noise in $\mathbf{x}$ for each fixed $\mathbf{t}$, one may wonder how the left-hand side of (4.20) could even make sense. In fact, everything is easily made rigorous: for smooth functions $\varphi$ and $\psi$ with compact support we define

$$
\mathbb{E}\left[\left\langle\varphi, \partial_{\mathbf{x}} \mathfrak{h}^{\varepsilon}(\mathbf{t}, \cdot)\right\rangle\left\langle\psi, \partial_{\mathbf{x}} \mathfrak{h}^{\varepsilon}(0, \cdot)\right\rangle\right]
$$

through

$$
\left\langle\varphi, \partial_{\mathbf{x}} \mathfrak{h}^{\varepsilon}(\mathbf{t}, \cdot)\right\rangle=-\int d \mathbf{x} \varphi^{\prime}(\mathbf{x}) \mathfrak{h}^{\varepsilon}(\mathbf{t}, \mathbf{x}) .
$$

From our results, they converge to

$$
\mathbb{E}\left[\left\langle\varphi, \partial_{\mathbf{x}} \mathfrak{h}(\mathbf{t}, \cdot)\right\rangle\left\langle\psi, \partial_{\mathbf{x}} \mathfrak{h}(0, \cdot)\right\rangle\right]
$$

From [25, equation (1.10)] they converge to $\left({ }^{31}\right)$

$$
\frac{1}{4} \int_{\mathbb{R}^{2}} d \mathbf{x} d \mathbf{y} \varphi\left(\frac{1}{2}(\mathbf{y}+\mathbf{x})\right) \psi\left(\frac{1}{2}(\mathbf{y}-\mathbf{x})\right) \mathbf{t}^{-2 / 3} g_{\mathrm{sc}}^{\prime \prime}\left(\mathbf{t}^{-2 / 3} \mathbf{x}\right) .
$$

This gives the equality (4.20) in the sense of distributions. But since the right-hand side is a regular function, the left is as well, and the two sides are equal.

The novelty over [25] is the existence of the stationary Markov process having this space-time covariance.

\section{Appendix A. Trace norm of the fixed point kernel}

If $K$ is an integral operator acting on the Hilbert space $\mathcal{H}=L^{2}(X, d \mu)$ through its kernel

$$
(K f)(x)=\int_{X} d \mu(y) K(x, y) f(y)
$$

(31) The $\frac{1}{4}$ prefactor comes from a minor correction in the final arXiv version of [25]; we thank Patrik Ferrari and Leandro Pimentel for bringing to our attention the correct scaling in this formula. 
its Fredholm determinant is defined by

$$
\operatorname{det}(I+K)=\sum_{n=0}^{\infty} \operatorname{tr}\left(\Lambda^{n}(K)\right)=\sum_{n=0}^{\infty} \frac{1}{n !} \int_{X^{n}} d \mu\left(x_{1}\right) \ldots d \mu\left(x_{n}\right) \operatorname{det}\left[K\left(x_{i}, x_{j}\right)\right]_{i, j=1}^{n},
$$

where $\Lambda^{n}(K)$ denotes the action of the $n$-fold tensor product $K \otimes \ldots \otimes K$ on the antisymmetric subspace of $\mathcal{H} \otimes \ldots \otimes \mathcal{H}$. One has $\operatorname{tr}\left(\Lambda^{n}(K)\right) \leqslant\left(\|K\|_{1}\right)^{n} / n$ !, where

$$
\|K\|_{1}=\operatorname{tr} \sqrt{K^{*} K}
$$

is the trace norm, so the Fredholm determinant is finite for trace class operators; in fact, it is also continuous with respect to the trace norm:

$$
|\operatorname{det}(I-A)-\operatorname{det}(I-B)| \leqslant\|A-B\|_{1} e^{1+\|A\|_{1}+\|B\|_{1}} .
$$

While the trace and the Fredholm determinant are invariant under conjugations $K \mapsto \Gamma^{-1} K \Gamma$, the trace norm is not. So, bounds on (and convergence in) trace norm, after appropriate conjugations, will allow us to justify the missing technical steps in $\S 3$ and $\S 4$. (For more background on the Fredholm determinant, including the definition and properties of the Hilbert-Schmidt and trace norms, we refer to [59] or [50, §2]).

\section{A.1. Proof of Theorem 4.1}

In this section, we prove that the kernel $\mathbf{K}_{\mathbf{t}, \mathrm{ext}}^{\mathrm{hypo}\left(\mathfrak{h}_{0}\right)}$ in the fixed point formula (4.7) is trace class (after conjugation by $\vartheta$, defined in (4.6)) and depends continuously on the initial data $\mathfrak{h}_{0} \in \mathrm{UC}$. The arguments in this section will provide us also with a blueprint for the much harder proof of the fact, to be used crucially in the proof of Proposition 3.6, that the approximating kernels from TASEP are trace class uniformly in the scaling parameter $\varepsilon$ (this is proved in Appendix B).

Our kernels appear in a number of different forms throughout the article. Since the approximating kernels from TASEP come naturally in the epi form we will prove the result for the epi version; the hypo version will just follow by reflection. We also have the continuum statistics formula of Theorem 4.4, which is apparently harder than the extended kernel formulas because those are always surrounded by explicit cutoffs $\chi_{\mathbf{a}}$ (in the hypo case; or $\bar{\chi}_{\mathbf{a}}$ in the epi case) but this one does not seem to have them; in a sense, in Theorem 4.4, the second $\mathbf{K}_{-\mathbf{t}}^{\mathrm{epi}(\mathfrak{g})}$ has to act as the cutoff. To see how this could work, use the definition of $\mathbf{K}_{\mathbf{t}}^{\text {epi }(\mathfrak{g})}$ given in Theorem 4.4, and for $f \in L^{2}(\mathbb{R})$ let $\Gamma f(u)=e^{G(u)} f(u)$, where $G$ is antisymmetric, i.e. it produces a cutoff satisfying $\Gamma^{-1} \varrho=\varrho \Gamma$, with $\varrho$ being the reflection operator $\varrho f(x)=f(-x)$. Then, after conjugating the kernel by $\Gamma$, we have 
(using the definition of the epi version of the Brownian scattering transform after (4.9), which gives $\left.\mathbf{K}_{-\mathbf{t}}^{\mathrm{epi}(\mathfrak{g})}=\left(\mathbf{K}_{\mathbf{t}}^{\mathrm{hypo}(-\varrho \mathfrak{g})}\right)^{*}\right)$

$$
\begin{aligned}
\Gamma \mathbf{K}_{\mathbf{t}}^{\text {hypo }(\mathfrak{h})} \mathbf{K}_{-\mathbf{t}}^{\text {epi }(\mathfrak{g})} \Gamma^{-1} & =\left(\Gamma \mathbf{K}_{\mathbf{t}}^{\text {hypo(h) }} \Gamma\right)\left(\Gamma^{-1}\left(\varrho \mathbf{K}_{\mathbf{t}}^{\text {hypo }(-\varrho \mathfrak{g})} \varrho\right)^{*} \Gamma^{-1}\right) \\
& =\left(\Gamma \mathbf{K}_{\mathbf{t}}^{\text {hypo(h) }} \Gamma\right)\left(\varrho \Gamma \mathbf{K}_{\mathbf{t}}^{\text {hypo }(-\varrho \mathfrak{g})} \Gamma \varrho\right)^{*} .
\end{aligned}
$$

Since the trace class operators form an ideal and $\varrho$ is bounded, it suffices to prove that $\Gamma \mathbf{K}_{\mathbf{t}}^{\text {hypo(h) }} \Gamma$ is trace class for $\mathfrak{h} \in \mathrm{UC}$. It will be clear from the argument that the cutoffs $\chi_{\mathbf{a}}$ can be replaced by such $\Gamma$ with $G(u)=\kappa \operatorname{sgn}(u)|u|^{3 / 2}$ with a sufficiently small $\kappa>0$, and we will not comment further on this.

The form of the kernel for $\mathfrak{g} \in \mathrm{LC}$ can be written explicity using the right-hand side of (4.5),

$$
\begin{aligned}
\mathbf{K}_{\mathbf{t}, \text { ext }}^{\mathrm{epi}(\mathfrak{g})}=- & e^{\left(\mathbf{x}_{j}-\mathbf{x}_{i}\right) \partial^{2}} \mathbf{1}_{\mathbf{x}_{i}<\mathbf{x}_{j}}+\left(\mathbf{S}_{-\mathbf{t}, \mathbf{x}_{0}-\mathbf{x}_{i}}^{\mathrm{epi}\left(\mathfrak{x}_{0},-\right.}\right){ }^{*} \mathbf{S}_{-\mathbf{t},-\mathbf{x}_{0}+\mathbf{x}_{j}} \\
& +\left(\mathbf{S}_{-\mathbf{t}, \mathbf{x}_{0}-\mathbf{x}_{i}}\right)^{*} \mathbf{S}_{-\mathbf{t},-\mathbf{x}_{0}+\mathbf{x}_{j}}^{\mathrm{epi}\left(\mathfrak{g}_{0},+\right.}-\left(\mathbf{S}_{-\mathbf{t}, \mathbf{x}_{0}-\mathbf{x}_{i}}^{\mathrm{epi}\left(\mathfrak{g}_{\mathbf{x}},-\right.}\right){ }^{*} \mathbf{S}_{-\mathbf{t},-\mathbf{x}_{0}+\mathbf{x}_{j}}^{\mathrm{epi}\left(\mathbf{g}_{0},+\right.},
\end{aligned}
$$

with $\mathbf{x}_{0}$ the splitting point. We want to prove that $\vartheta \bar{\chi}_{\mathbf{a}} \mathbf{K}_{\mathbf{t}, \text { ext }}^{\text {epi }(\mathfrak{g})} \bar{\chi}_{\mathbf{a}} \vartheta^{-1}$ is trace class. We will show that each of the three last terms is trace class after surrounding by $\bar{\chi}_{\mathbf{a}}$. The argument for the first term using the conjugation by $\vartheta$ is in [9, Lemma A.2] $\left({ }^{32}\right)$ and works the same way here. In the other terms, one can check through the argument that the conjugation by $\vartheta$ does not present any real difficulty, so to make the proof readable we leave them out. Note also that, by shifting the height and rescaling $\mathfrak{h}$, we may assume that $\mathbf{a}=0$ and $\mathbf{t}=1$. We will always assume this in the proof, in order to make it easier to follow.

The proof uses the classical bound on the Airy functions, $|\operatorname{Ai}(x)| \leqslant C e^{-2 / 3(x \vee 0)^{3 / 2}}$, which in our context yields

$$
\left|\mathbf{S}_{-1, \mathbf{x}}(u)\right| \leqslant C \exp \widehat{F}_{0}(\mathbf{x}, u)
$$

with

$$
\widehat{F}_{0}(\mathbf{x}, u)=\mathbf{x y}-\frac{1}{3} \mathbf{x}^{3}-\frac{2}{3}(\mathbf{y} \vee 0)^{3 / 2} \text { and } \quad \mathbf{y}=\mathbf{x}^{2}+u .
$$

By checking various cases, it is elementary to see from (A.5) that we also have

$$
\int_{-\infty}^{0} d \eta\left|\mathbf{S}_{-1, \mathbf{x}}(u-\eta)\right|^{2} \leqslant C \exp \left\{2 F_{0}(\mathbf{x}, u)\right\}
$$

$\left({ }^{32}\right)$ Note that there is a typo in the statement of this result, where the ratio corresponding to the $\vartheta_{i}$ 's should be inverted. 
where $F_{0}=\widehat{F}_{0}$ unless $\mathbf{x}>0$ and $u<0$, in which case $F_{0}=0$, or $\mathbf{x}=0$ and $u<0$, in which case the bound is $F_{0}=\log (1+|u|)$. Note that the constant $C$, and all the bounds here, do depend on $\mathbf{x}$.

It is enough to control the trace norm of the third term in (A.4), since the second term takes the form of a transpose of that one, and the fourth term is the product of two such terms. Call $\mathbf{x}_{0}-\mathbf{x}_{i}=\mathbf{x}_{1}$ and $-\mathbf{x}_{0}+\mathbf{x}_{j}=-\mathbf{x}_{2}$. We write our kernel explicitly as

$$
\begin{aligned}
& \left(\mathbf{S}_{-1, \mathbf{x}_{1}}\right)^{*} \mathbf{S}_{-1,-\mathbf{x}_{2}}^{\mathrm{epi}\left(\mathfrak{g}^{\mathbf{x},+}\right)}\left(z_{1}, z_{2}\right) \\
& \quad=\int_{\substack{\mathbf{s} \geqslant 0 \\
\mathbf{b}, z \in \mathbb{R}}} d z \mathbb{P}_{\mathbf{B}(0)=z}(\boldsymbol{\tau} \in d \mathbf{s}, \mathbf{B}(\boldsymbol{\tau}) \in d \mathbf{b}) \mathbf{S}_{-1, \mathbf{x}_{1}}\left(z, z_{1}\right) \mathbf{S}_{-1,-\mathbf{x}_{2}-\mathbf{s}}\left(\mathbf{b}, z_{2}\right),
\end{aligned}
$$

where $\boldsymbol{\tau}$ is the hitting time of epi $\left(\mathfrak{g}^{\mathbf{x},+}\right)$. We can think of the right-hand side as an integral of operator kernels in $z_{1}$ and $z_{2}$ over some extra parameters $z, \mathbf{b}$ and $\mathbf{s}$, and we can estimate its trace norm $\|\cdot\|_{1}$ by the integral of those trace norms:

$$
\begin{aligned}
& \left\|\left(\mathbf{S}_{-1, \mathbf{x}_{1}}\right)^{*} \mathbf{S}_{-1,-\mathbf{x}_{2}}^{\mathrm{epi}\left(\mathfrak{g}^{\mathbf{x},+}\right)}\right\|_{1} \\
& \quad \leqslant \int_{\substack{\mathbf{s} \geqslant 0 \\
\mathbf{b}, z \in \mathbb{R}}} d z \mathbb{P}_{\mathbf{B}(0)=z}(\boldsymbol{\tau} \in d \mathbf{s}, \mathbf{B}(\boldsymbol{\tau}) \in d \mathbf{b})\left\|\mathbf{S}_{-1, \mathbf{x}_{1}}\left(z, z_{1}\right) \mathbf{S}_{-1,-\mathbf{x}_{2}-\mathbf{s}}\left(\mathbf{b}, z_{2}\right)\right\|_{1} .
\end{aligned}
$$

The advantage of the expression on the right-hand side is that it isolates very clearly the dependence of the trace norm on the function $\mathfrak{g}$ through the Brownian hitting time and position (see also Remark A.1). Because of the cutoffs $\bar{\chi}_{\mathbf{a}}, \mathbf{a}=0$, the trace norm is computed on $L^{2}((-\infty, 0])$. The operator inside the norm above has rank 1 , so its trace norm is now just the product of $L^{2}$ norms, and using also (A.6), we get

$$
\begin{aligned}
& \left\|\mathbf{S}_{-1, \mathbf{x}_{1}}\left(z, z_{1}\right) \mathbf{S}_{-1,-\mathbf{x}_{2}-\mathbf{s}}\left(\mathbf{b}, z_{2}\right)\right\|_{1} \\
& \quad=\sqrt{\int_{-\infty}^{0} d z_{1}\left|\mathbf{S}_{-1, \mathbf{x}_{1}}\left(z-z_{1}\right)\right|^{2} \int_{-\infty}^{0} d z_{2}\left|\mathbf{S}_{-1,-\mathbf{x}_{2}-\mathbf{s}}\left(\mathbf{b}, z_{2}\right)\right|^{2}} \\
& \quad \leqslant C \exp \left\{F_{0}\left(\mathbf{x}_{1}, z\right)+F_{0}\left(-\mathbf{x}_{2}-\mathbf{s}, \mathbf{b}\right)\right\} .
\end{aligned}
$$

Now, we use our key assumption $\mathfrak{g}(\mathbf{x}) \geqslant-\overline{\boldsymbol{\alpha}}-\bar{\gamma}|\mathbf{x}|$. Observing from $\mathbf{x}_{0}$, we have

$$
\mathfrak{g}\left(\mathbf{x}_{0}+\mathbf{x}\right) \geqslant-\overline{\boldsymbol{\alpha}}-\bar{\gamma}\left|\mathbf{x}_{0}+\mathbf{x}\right| \geqslant-\widetilde{\boldsymbol{\alpha}}-\widetilde{\gamma}|\mathbf{x}|
$$

for some new positive constants, which do depend on the $\mathbf{x}_{i}$. So, we obtain

$$
\mathbf{b} \geqslant-\widetilde{\boldsymbol{\alpha}}-\widetilde{\gamma} \mathbf{s}
$$

From this, it is not hard to see that there are constants $\kappa_{1}>0$ and $C<\infty$ depending on $\mathbf{x}_{2}$ such that

$$
F_{0}\left(-\mathbf{x}_{2}-\mathbf{s}, \mathbf{b}\right) \leqslant-\kappa_{1} \mathbf{s}^{3}+C .
$$


Furthermore, there is a $C<\infty$ depending on $\mathbf{x}_{1}$ such that

$$
F_{0}\left(\mathbf{x}_{1}, z\right) \leqslant C+C|z|^{3 / 2} \mathbf{1}_{z \leqslant-\widetilde{\boldsymbol{\alpha}}}-\frac{1}{3}|z|^{3 / 2} \mathbf{1}_{z \geqslant-\tilde{\boldsymbol{\alpha}}} .
$$

Let $\boldsymbol{\sigma}$ be the hitting time of the epigraph of $-\widetilde{\boldsymbol{\alpha}}-\widetilde{\boldsymbol{\gamma}}|\mathbf{x}|$ by the Brownian motion $\mathbf{B}$. Clearly, $\boldsymbol{\tau} \geqslant \boldsymbol{\sigma}$. We have $\mathbb{P}_{z}(\boldsymbol{\sigma} \leqslant \mathbf{s})=\mathbb{P}_{z}\left(\sup _{0 \leqslant \mathbf{x} \leqslant \mathbf{s}} \mathbf{B}(\mathbf{x})+\widetilde{\boldsymbol{\alpha}}+\widetilde{\boldsymbol{\gamma}} \mathbf{x}>0\right)$. For $z<-\widetilde{\boldsymbol{\alpha}}$, we can bound this by $\mathbb{P}_{z}\left(\sup _{0 \leqslant \mathbf{x} \leqslant \mathbf{s}} \mathbf{B}(\mathbf{x})+\widetilde{\boldsymbol{\alpha}}+\widetilde{\boldsymbol{\gamma}} \mathbf{s}>0\right)$, which can be computed by the reflection principle, to give the bound

$$
\mathbb{P}_{z}(\boldsymbol{\tau} \leqslant \mathbf{s}) \leqslant C \exp \left\{-\kappa_{2} \mathbf{s}^{-1}(z+\widetilde{\boldsymbol{\alpha}}+\widetilde{\boldsymbol{\gamma}} \mathbf{s})^{2}\right\}
$$

Putting it all together, we have that $\left\|\left(\mathbf{S}_{-1, \mathbf{x}_{1}}\right)^{*} \mathbf{S}_{-1,-\mathbf{x}_{2}}^{\mathrm{epi}\left(\mathfrak{g}^{\mathbf{x},+}\right)}\right\|_{1}$ is bounded by a constant multiple of

$$
\int_{-\infty}^{-\widetilde{\boldsymbol{\alpha}}} d z \int_{0}^{\infty} d \mathbf{s} e^{-\kappa_{2} \mathbf{s}^{-1}(z+\widetilde{\boldsymbol{\alpha}}+\widetilde{\mathbf{\gamma}} \mathbf{s})^{2}+C|z|^{3 / 2}-\kappa_{1} \mathbf{s}^{3}}+\int_{-\widetilde{\boldsymbol{\alpha}}}^{\infty} d z \int_{0}^{\infty} d \mathbf{s} e^{-|z|^{3 / 2} / 3-\kappa_{1} \mathbf{s}^{3}},
$$

which converge. This finishes proving that $\vartheta \bar{\chi}_{\mathbf{a}} \mathbf{K}_{\mathbf{t}, \text { ext }}^{\text {epi }(\mathfrak{g})} \bar{\chi}_{\mathbf{a}} \vartheta^{-1}$ is trace class.

The method also allows us to show continuity with respect to $\mathfrak{g} \in \mathrm{LC}$ of the above kernel, which yields Theorem 4.1. In fact, consider a sequence of functions $\left(\mathfrak{g}_{n}\right)_{n \geqslant 0}$ converging to $\mathfrak{g}$ in LC. The measures $\mathbb{P}_{\mathbf{B}(0)=z}\left(\boldsymbol{\tau}^{n} \in d \mathbf{s}, \mathbf{B}\left(\boldsymbol{\tau}^{n}\right) \in d \mathbf{b}\right)$, where $\boldsymbol{\tau}^{n}$ is the hitting time of epi $\left(\mathfrak{g}^{n}\right)$, converge to $\mathbb{P}_{\mathbf{B}(0)=z}(\boldsymbol{\tau} \in d \mathbf{s}, \mathbf{B}(\boldsymbol{\tau}) \in d \mathbf{b})$ as $n \rightarrow \infty$, analogously to the last paragraph of the proof of Lemma 3.5. This can be used, together with the above estimates, to show that

$$
\int_{\substack{\mathbf{s} \geqslant 0 \\ \mathbf{b}, z \in \mathbb{R}}} d z \mathbb{P}_{\mathbf{B}(0)=z}\left(\boldsymbol{\tau}^{n} \in d \mathbf{s}, \mathbf{B}\left(\boldsymbol{\tau}^{n}\right) \in d \mathbf{b}\right) \mathbf{S}_{-1, \mathbf{x}_{1}}\left(z, z_{1}\right) \mathbf{S}_{-1,-\mathbf{x}_{2}-\mathbf{s}}\left(\mathbf{b}, z_{2}\right)
$$

converges in trace norm to

$$
\int_{\substack{\mathbf{s} \geqslant 0 \\ \mathbf{b}, z \in \mathbb{R}}} d z \mathbb{P}_{\mathbf{B}(0)=z}(\boldsymbol{\tau} \in d \mathbf{s}, \mathbf{B}(\boldsymbol{\tau}) \in d \mathbf{b}) \mathbf{S}_{-1, \mathbf{x}_{1}}\left(z, z_{1}\right) \mathbf{S}_{-1,-\mathbf{x}_{2}-\mathbf{s}}\left(\mathbf{b}, z_{2}\right) ;
$$

see $§$ B.2 (and in particular Proposition B.6), where this argument is implemented in the more complicated case of convergence of the TASEP kernels to their fixed point limit.

Remark A.1. Control of the Fredholm determinant is usually achieved either by Hadamard's inequality, or through a trace-norm estimate. Many articles in the field skip this step and only prove pointwise convergence of the integral kernel. Earlier complete arguments were in special cases where all objects in an equation like (A.7) were completely explicit, and Hadamard's inequality is easier to apply. The trace norm is natural 
(and yields precise estimates) for Fredholm determinants due to (A.2), but has the disadvantage that it is in general difficult to compute. Usually, one tries to write the operator as product $K=A B$, and bound the trace norm by the product of the Hilbert-Schmidt norms of its factors $\|A B\|_{1} \leqslant\|A\|_{2}\|B\|_{2}$, the latter being easy to compute. In our case the left-hand side of (A.7) is obviously a product, but one has to take

$$
A=\left(\mathbf{S}_{-1, \mathbf{x}_{1}}\right)^{*} T^{-1} \quad \text { and } \quad B=T \mathbf{S}_{-1,-\mathbf{x}_{2}}^{\mathrm{epi}\left(\mathfrak{g}^{\mathbf{x},+}\right)},
$$

where $T$ is a carefully chosen multiplication operator, depending heavily on the initial data and $\mathbf{x}$ and $z$ through $\mathbb{P}_{\mathbf{B}(0)=z}(\boldsymbol{\tau} \in d \mathbf{s}, \mathbf{B}(\boldsymbol{\tau}) \in d \mathbf{b})$. While writing a proof along these lines, we noticed that it was suggesting that we could just take the trace norm inside the integration, as in (A.8). It is far from obvious, but true, that the estimate does not give away too much. Once inside, the trace norm is that of a rank-1 operator, and easy to compute. The resulting method, besides working in general, is much easier than earlier proofs.

\section{A.2. Proof of Proposition 4.3}

In order to check the path integral formula (4.8), we will apply [8, Theorem 3.3] to the extended kernel formula $\operatorname{det}\left(\mathbf{I}-\chi_{\mathbf{a}} \widetilde{\mathbf{K}}_{\mathbf{t}, \text { ext }}^{\text {hypo }}{ }^{\left(\mathfrak{h}_{0}\right)} \chi_{\mathbf{a}}\right)$, where $\widetilde{\mathbf{K}}_{\mathbf{t}, \text { ext }}^{\text {hypo }}\left(\mathfrak{h}_{0}\right)$ is the conjugated kernel $\vartheta \mathbf{K}_{\mathbf{t} \text {,ext }}^{\text {hypo }\left(\mathfrak{h}_{0}\right)} \vartheta^{-1}$. Here, we are using the fact that $\vartheta$ and $\vartheta^{-1}$ commute with $\chi_{\mathbf{a}}$ to see that this determinant is the same as the one in (4.7); the conjugation by $\vartheta$ will enable us to check that the analytical assumptions in the [8] result are satisfied. In the notation of that theorem, we have $Q_{\mathbf{x}_{i}}=\chi_{\mathbf{a}_{i}}$ as well as $\mathcal{W}_{\mathbf{x}_{i}, \mathbf{x}_{j}}=\vartheta_{i} e^{\left(\mathbf{x}_{j}-\mathbf{x}_{i}\right) \partial^{2}} \vartheta_{j}^{-1}$ for $\mathbf{x}_{i}<\mathbf{x}_{j}$, $K_{\mathbf{x}_{i}}=\vartheta_{i} e^{-\mathbf{x}_{i} \partial^{2}} \mathbf{K}_{\mathbf{t}}^{\text {hypo(ho) }} e^{\mathbf{x}_{i} \partial^{2}} \vartheta_{i}^{-1}$ and $\mathcal{W}_{\mathbf{x}_{j}, \mathbf{x}_{i}} K_{\mathbf{x}_{i}}=\vartheta_{j} e^{-\mathbf{x}_{j} \partial^{2}} \mathbf{K}_{\mathbf{t}}^{\text {hypo }\left(\mathfrak{h}_{0}\right)} e^{\mathbf{x}_{i} \partial^{2}} \vartheta_{i}^{-1}$ for $\mathbf{x}_{i}<\mathbf{x}_{j}$. Additionally, we set $V_{\mathbf{x}_{i}}=\mathbf{I}, V_{\mathbf{x}_{i}}^{\prime}=\mathbf{I}, U_{\mathbf{x}_{i}}=\Gamma$ and $U_{\mathbf{x}_{i}}^{\prime}=\Gamma^{-1}$ (see (A.3)). Note, however, that in [8] the operators $Q_{t_{i}}$ appear multiplying only on the left of $\mathbf{K}_{\mathbf{t}, \text { ext }}^{\text {hypo(ho }}{ }^{\text {. }}$. While we could use the cyclic property of the determinant to remove the second projection $\chi_{\mathbf{a}}$ in our extended kernel formula, it is more convenient to leave it there and note instead that $[8$, Theorem 3.3] applies in this case just as well, with only a minor modification: assuming that the operators $Q_{t_{i}}$ appearing in [8] have a square root, then Assumption 1 (i) of the theorem is now the boundedness in $L^{2}(\mathbb{R})$ of $Q_{t_{i}}^{1 / 2} \mathcal{W}_{t_{i}, t_{j}} Q_{t_{j}}^{1 / 2}$ for $i<j$, and of $Q_{t_{i}}^{1 / 2} K_{t_{i}} Q_{t_{i}}^{1 / 2}$ and $Q_{t_{j}}^{1 / 2} \mathcal{W}_{t_{j}, t_{i}} K_{t_{j}} Q_{t_{j}}^{1 / 2}$ for all $i$ and $j$, and similarly Assumption 3 (ii) is the fact that the same operators are trace class when surrounded by $V_{t_{i}}$ and $V_{t_{i}}^{\prime}$ (these modifications are analogous to part of what we do in Appendix D.1, where we multiply by $N^{1 / 2}$ on both sides in the left-hand side of (D.4), and their validity can be checked simply by inspecting the proof of Theorem D.1). 
We turn now to checking that the three assumptions of [8, Theorem 3.3] hold in our setting. Assumption 3 (ii) (with the modification discussed above) corresponds exactly to the verifying that each of the entries $\chi_{\mathbf{a}} \widetilde{\mathbf{K}}_{\mathbf{t}, \text { ext }}^{\text {hypo }\left(\mathfrak{h}_{0}\right)} \chi_{\mathbf{a}}\left(\mathbf{x}_{i}, \cdot ; \mathbf{x}_{j}, \cdot\right)$ of our extended kernel are trace class in $L^{2}(\mathbb{R})$, which is what we just proved above. This also yields (the modified) Assumption 1 (i), which corresponds to asking only that these operators are bounded. Assumption 1 (ii), on the other hand, actually does not hold in our setting. We note, however, that the assumption is never really used in the proof of [8, Theorem 3.3]. In fact, the assumption appears there only because that paper worked in a setting where all Fredholm determinants under consideration involved bounded operators in $L^{2}(\mathbb{R})$, but all that actually matters in the proof is that the operator in that assumption is trace class after an appropriate conjugation, and this is exactly the content of Assumption 3 (iii), which can be seen to hold using the above arguments (see the comment after (A.3)). Assumption 3 (i) holds trivially. Finally, Assumption 2 follows directly from the definition of $\mathbf{K}_{\mathbf{t}}^{\text {hypo(ho) }}$ and the group property of the operators $\mathbf{S}_{\mathbf{t}, \mathbf{x}}$.

Having checked all the assumptions, we may now apply the [8] result, which yields the path integral formula (4.8) conjugated by $\vartheta_{1}^{-1}$.

\section{Appendix B. Trace-norm convergence of the rescaled TASEP kernels}

\section{B.1. Estimates}

In this section, we obtain uniform in $\varepsilon$ bounds on the trace norm of the discrete approximations of the fixed point kernel. We always assume that $\mathfrak{g}^{\varepsilon} \rightarrow \mathfrak{g}$ in LC and, in particular, that they satisfy the linear bound $\mathfrak{g}^{\varepsilon}(\mathbf{y}), \mathfrak{g}(\mathbf{y}) \geqslant-\overline{\boldsymbol{\alpha}}-\bar{\gamma}|\mathbf{y}|$ for $\mathbf{y} \in \mathbb{R}$, uniformly in $\varepsilon$.

The proof somewhat follows the lines of the continuum version, but there are several new difficulties. The first is that the continuum proof used many asymptotics of the functions $\mathbf{S}_{-\mathbf{t}, \mathbf{x}}$, each of which has to be done separately now using steepest descent on the contour integrals defining the functions $\mathbf{S}_{-\mathbf{t}, \mathbf{x}}^{\varepsilon}$ and $\overline{\mathbf{S}}_{-\mathbf{t}, \mathbf{x}}^{\varepsilon}$. A more serious problem is that we do not have a split formula at the TASEP level, i.e. a formula of the type (3.25). In other words, we do not really have a usable formula for 2-sided data for TASEP. Such a formula appeared in the first version of this article on the arXiv, but it does not seem to be usable, and, in particular, we have not succeeded so far to employ it to control the trace norm of the kernel. Because of this, and as we discussed in $\S 3$, we need estimates for the TASEP kernel for the LC cutoffs of $\mathfrak{g}^{\varepsilon}$ at $L<\infty$ (see Definition 3.3), uniformly in $L$, so that the cutoff can later be removed (through the finite propagation speed result proved in Appendix C.1). The same type of estimates are needed to prove the uniform bounds on the Hölder norms of the fixed point (see Appendix C.2). More precisely, we need to prove the following result. 
Proposition B.1. Consider $\mathfrak{g}^{\varepsilon}$ as above and let $\mathfrak{g}_{L}^{\varepsilon}$ denote its LC cutoff at $L>0$. Then, the trace norm of $\left(\mathbf{S}_{-\mathbf{t}, \mathbf{x}_{1}-L}^{\varepsilon}\right)^{*} \overline{\mathbf{S}}_{-\mathbf{t},-\mathbf{x}_{2}+L}^{\left.\left.\varepsilon, \text { epi( } \mathfrak{g}_{L}^{\varepsilon}\right)^{L,-}\right)}$ on $L^{2}((-\infty, \mathbf{a}])$ is bounded uniformly in $\varepsilon$ and $L$.

By definition of the notation $\mathfrak{g}^{L,-}$ (see (4.3)) we have $\left(\mathfrak{g}_{L}^{\varepsilon}\right)^{L,-}(\mathbf{y})=\left(\mathfrak{g}^{\varepsilon}\right)^{L,-}(\mathbf{y})$ for all $\mathbf{y} \geqslant 0$, so in the proposition we could have written $\mathfrak{g}^{\varepsilon}$ without the cutoff. We have chosen this formulation to stress the role of $L$ in this result and our interest in it.

From the proof of the continuum case, one can see already that proving this is going to be difficult. In the proof, we first take care of the case $L=0$ and then, after (B.9), extend to all $L>0$, using that crucial identity as the main tool.

From the continuum proof, we see that the key point is to bound the trace norm by the integral of trace norms of rank- 1 operators, which become $L^{2}$ norms. So, we introduce the notation

$$
\exp 2 F_{\varepsilon}(\mathbf{x}, u)=\int_{-\infty}^{0} d \eta\left|\mathbf{S}_{-1, \mathbf{x}}^{\varepsilon}(u-\eta)\right|^{2} \quad \text { and } \quad \exp 2 \bar{F}_{\varepsilon}(\mathbf{x}, u)=\int_{-\infty}^{0} d \eta\left|\overline{\mathbf{S}}_{-1, \mathbf{x}}^{\varepsilon}(u-\eta)\right|^{2},
$$

Here and below, we write $\mathbf{S}_{-1, \mathbf{x}}^{\varepsilon}(u-z)=\mathbf{S}_{-1, \mathbf{x}}^{\varepsilon}(u, z)$ and $\overline{\mathbf{S}}_{-1, \mathbf{x}}^{\varepsilon}(u-z)=\overline{\mathbf{S}}_{-1, \mathbf{x}}^{\varepsilon}(u, z)$. Going back to (2.27) and (2.28) to compute these integrals as summations, we obtain

$$
\int_{-\infty}^{0} d \eta\left|\mathbf{S}_{-1, \mathbf{x}}^{\varepsilon}(u-\eta)\right|^{2}=\frac{1}{(2 \pi i)^{2}} \oint_{C_{\varepsilon}^{o}} d \widetilde{w}_{1} \oint_{C_{\varepsilon}^{o}} d \widetilde{w}_{2} \frac{e^{\varepsilon^{-3 / 2} \sum_{i=1}^{2} F\left(\varepsilon^{1 / 2} \widetilde{w}_{i}, \varepsilon^{1 / 2} \mathbf{x}_{\varepsilon}, \varepsilon u_{\varepsilon}\right)}}{\widetilde{w}_{1}+\widetilde{w}_{2}-\varepsilon^{1 / 2} \widetilde{w}_{1} \widetilde{w}_{2}},
$$

with $\mathbf{x}_{\varepsilon}=\mathbf{x}-\frac{1}{2} \varepsilon^{1 / 2} u-\frac{1}{2} \varepsilon$ and $u_{\varepsilon}=u-\varepsilon^{1 / 2}$, and $F$ defined in (3.20), as well as

$$
\begin{aligned}
& \int_{-\infty}^{0} d \eta\left|\overline{\mathbf{S}}_{-1, \mathbf{x}}^{\varepsilon}(u-\eta)\right|^{2} \\
& =\frac{1}{(2 \pi i)^{2}} \oint_{C_{\varepsilon}^{o}} d \widetilde{w}_{1} \oint_{C_{\varepsilon}^{o}} d \widetilde{w}_{2} \frac{e^{\varepsilon^{-3 / 2} \sum_{i=1}^{2} F\left(\varepsilon^{1 / 2} \widetilde{w}_{i}, \varepsilon^{1 / 2} \overline{\mathbf{x}}_{\varepsilon}, \varepsilon \bar{u}_{\varepsilon}\right)}\left(1+\varepsilon^{1 / 2} \widetilde{w}_{1}\right)\left(1+\varepsilon^{1 / 2} \widetilde{w}_{2}\right)}{\widetilde{w}_{1}+\widetilde{w}_{2}+\varepsilon^{1 / 2} \widetilde{w}_{1} \widetilde{w}_{2}},
\end{aligned}
$$

with $\overline{\mathbf{x}}_{\varepsilon}=\mathbf{x}+\frac{1}{2} \varepsilon^{1 / 2} u+\frac{3}{2} \varepsilon$ and $\bar{u}_{\varepsilon}=u+\varepsilon^{1 / 2}$; the notation $C_{\varepsilon}^{o}$ means that the singularity at zero is outside the contour.

The following lemma replaces (A.6) in the discrete case. The function $\widehat{F}_{0}$ is replaced by $F\left(\mathbf{w}_{+}, \mathbf{x}, u\right)$, which we call

$$
\begin{aligned}
& \widehat{F}_{\varepsilon}(\mathbf{x}, u) \\
& \quad=\operatorname{Re}\left[\left(1+\nu_{1}\left(\varepsilon^{1 / 2} \mathbf{w}_{+}\right)\right)\left(\mathbf{x y}-\frac{1}{3} \mathbf{x}^{3}-\frac{2}{3} \mathbf{y}^{3 / 2}\right)+\nu_{2}\left(\varepsilon^{1 / 2} \mathbf{w}_{+}\right)\left(\mathbf{x y}-\frac{1}{2} \mathbf{x}^{2} \mathbf{y}^{1 / 2}-\frac{1}{2} \mathbf{y}^{3 / 2}\right)\right],
\end{aligned}
$$

where $\mathbf{w}_{+}=-\mathbf{x}+\sqrt{\mathbf{y}}$, as before $\mathbf{y}=\mathbf{x}^{2}+u$, and

$$
\begin{aligned}
& \nu_{1}(\mathbf{w})=\mathbf{w}^{-3}\left(3\left(1+\mathbf{w}^{2}\right) \operatorname{arctanh} \mathbf{w}+3 \mathbf{w} \log \left(1-\mathbf{w}^{2}\right)-3 \mathbf{w}\right)-1, \\
& \nu_{2}(\mathbf{w})=\mathbf{w}^{-3}\left(-2\left(3+\mathbf{w}^{2}\right) \operatorname{arctanh} \mathbf{w}-4 \mathbf{w} \log \left(1-\mathbf{w}^{2}\right)+6 \mathbf{w}\right) .
\end{aligned}
$$


These two functions are analytic in $\mathbf{w} \in \mathbb{C} \backslash(-\infty,-1] \cup[1, \infty)$, uniformly bounded in absolute value everywhere, vanish at zero like $\mathbf{w}^{2}$, and are non-negative on $(-1,1)$, since they have convergent series expansions

$$
\nu_{1}(\mathbf{w})=\sum_{\substack{n \geqslant 2 \\ n \text { even }}} \frac{6}{(n+1)(n+2)(n+3)} \mathbf{w}^{n} \quad \text { and } \quad \nu_{2}(\mathbf{w})=\sum_{\substack{n \geqslant 2 \\ n \text { even }}} \frac{4 n}{(n+1)(n+2)(n+3)} \mathbf{w}^{n}
$$

there. Here and below, $\sqrt{\mathbf{y}}$ always refers to the positive square root.

The following lemma covers different regions in the asymptotics of the functions $F_{\varepsilon}(\mathbf{x}, u)$ and $\bar{F}_{\varepsilon}(\mathbf{x}, u)$. Unfortunately, there does not appear to be one argument which covers all regions, as we have complicated functions of several variables converging in $\varepsilon$. On the other hand, we do not need all regions and the estimates we actually need are far from the optimal ones. $\left({ }^{33}\right)$

LEMmA B.2. In the following all constants are independent of everything including $\varepsilon$, unless noted.

(i) Suppose that $\mathbf{x}_{\varepsilon}^{2}+u_{\varepsilon} \geqslant 0$ and $-\mathbf{x}_{\varepsilon}+\sqrt{\mathbf{x}_{\varepsilon}^{2}+u_{\varepsilon}} \geqslant \varepsilon^{-1 / 2}$. Then, $F_{\varepsilon}(\mathbf{x}, u)=-\infty$.

(ii) Suppose that $\mathbf{x}_{\varepsilon}^{2}+u_{\varepsilon} \geqslant 0$. There is $\delta>0$ such that, for

$$
-\delta \leqslant-\mathbf{x}_{\varepsilon}+\sqrt{\mathbf{x}_{\varepsilon}^{2}+u_{\varepsilon}}<\varepsilon^{-1 / 2},
$$

and $\varepsilon^{1 / 2} \mathbf{x}_{\varepsilon} \in(1-\sqrt{5}, 1+\sqrt{5})$, we have $F_{\varepsilon}(\mathbf{x}, u) \leqslant \widehat{F}_{\varepsilon}\left(\mathbf{x}_{\varepsilon}, u_{\varepsilon}\right)$. Under the same conditions on $\overline{\mathbf{x}}_{\varepsilon}$ and $\bar{u}_{\varepsilon}$, we have $\bar{F}_{\varepsilon}(\mathbf{x}, u) \leqslant \widehat{F}_{\varepsilon}\left(\overline{\mathbf{x}}_{\varepsilon}, \bar{u}_{\varepsilon}\right)$.

(iii) Suppose that $\mathbf{x}, \mathbf{x}_{\varepsilon} \geqslant 0$. Then, $F_{\varepsilon}(\mathbf{x}, u) \leqslant C \log (2+|u|+|\mathbf{x}|)$. The same holds for $\bar{F}_{\varepsilon}(\mathbf{x}, u)$ under the conditions $\overline{\mathbf{x}}_{\varepsilon} \geqslant 0$ and $u \geqslant-\varepsilon^{-1 / 2} \mathbf{x}$.

(iv) Suppose that $\mathbf{x}_{\varepsilon}^{2}+u_{\varepsilon}<0,-C \varepsilon^{-1 / 4} \leqslant \mathbf{x}_{\varepsilon}<0$ and $\left|u_{\varepsilon}\right| \leqslant C \varepsilon^{-1 / 2}$. Then, there is $C^{\prime}$ depending on $\left|u_{\varepsilon}\right|^{1 / 2} /\left|\mathbf{x}_{\varepsilon}\right|$ such that, for any $\delta>0$,

$$
F_{\varepsilon}(\mathbf{x}, u) \leqslant-\left(\frac{2}{3}-\delta\right)|\mathbf{x}|^{3}+C \delta^{-1}|u|^{3 / 2}+C^{\prime}
$$

(v) Suppose that $\overline{\mathbf{x}}_{\varepsilon}^{2}+\bar{u}_{\varepsilon}<0,-C<\overline{\mathbf{x}}_{\varepsilon}<0$, and $\left|\bar{u}_{\varepsilon}\right| \leqslant C$. Then, $\bar{F}_{\varepsilon}(\mathbf{x}, u) \leqslant C$.

$\left({ }^{33}\right)$ The estimates and arguments which we require in this lemma and in the rest of this section are much more involved than those appearing in earlier proofs of convergence to the classical Airy processes. One reason for this is that, whereas exact contour integral formulas were available in those special cases, our formulas involve expectations over random walk hitting times, and in order to handle them, we need to control the behavior of the contour integrals in some additional, complicated regions. But even if this were not a problem, we are in a situation where we need to obtain much finer estimates on our integrals in order to prove the uniform bounds on the Hölder norms of the fixed point, which play a crucial role in our arguments. 
Proof. Recall that, in (B.1) and (B.2) the contour $C_{\varepsilon}^{o}$ is $C_{\varepsilon}$, a circle of radius $\varepsilon^{-1 / 2}$ centered at $\varepsilon^{-1 / 2}$, with a little blip taken at its left so that zero lies outside the contour. Recall also that the function $F$ appearing in the exponents there is given by

$$
F(w, x, u)=\operatorname{arctanh} w-w-x \log \left(1-w^{2}\right)-u \operatorname{arctanh} w .
$$

We note that the real part of this function is symmetric in the imaginary part of $w$, so in the proof it will be enough to estimate the integrand along the upper half of the contour.

Proof of (i). Squaring both sides of $\sqrt{\mathbf{x}_{\varepsilon}^{2}+u_{\varepsilon}} \geqslant \varepsilon^{-1 / 2}+\mathbf{x}_{\varepsilon}$ and using $\mathbf{x}_{\varepsilon}^{2}+u_{\varepsilon} \geqslant 0$ gives

$$
\alpha_{\varepsilon}:=\frac{1}{2} \varepsilon^{-3 / 2}+\varepsilon^{-1} \mathbf{x}_{\varepsilon}-\frac{1}{2} \varepsilon^{-1 / 2} u_{\varepsilon} \leqslant 0 .
$$

But looking at the contour integral defining $\mathbf{S}_{-1, \mathbf{x}}^{\varepsilon}(u)$ through (3.15) and (2.27), we see that the pole at $w=0$ disappears exactly when $\alpha_{\varepsilon} \leqslant 0$, which shows that the integrand is analytic, and thus $\mathbf{S}_{-1, \mathbf{x}}^{\varepsilon}(u)=0$ in this case.

Proof of (ii). We are trying to estimate (B.1) and (B.2), and the term in the exponent is the same, except in one case evaluated at $\mathbf{x}_{\varepsilon}$ and $u_{\varepsilon}$, and in the other at $\overline{\mathbf{x}}_{\varepsilon}$ and $\bar{u}_{\varepsilon}$. So, the proofs will be the same and we just call the value in the exponent $\widetilde{\mathbf{x}}_{\varepsilon}$ and $\tilde{u}_{\varepsilon}$ to stand for one or the other (we will use this convention also in the proof of the other cases). We deform the contour $C_{\varepsilon}$ to a contour $\overline{\mathbf{w}}_{+}^{\varepsilon}+r e^{ \pm i \pi / 4}$, with $r$ going from zero until it hits the right arc of the old contour $C_{\varepsilon}$ (so that $r \in\left[0, c \varepsilon^{-1 / 2}\right]$ with $c \sim \sqrt{2}$ ), together with that right arc of the old contour $C_{\varepsilon}$ with angles $\leqslant \frac{1}{4} \pi$. Note that, during the deformation, we do not pass through any zeros of the denominator in either (B.1) or (B.2). The contour we have described is a "steep descent curve", in the sense that it is close enough to the steepest descent curve for our purposes. In this case, it does actually pass through the critical point $\mathbf{w}_{+}^{\varepsilon}$. Our estimate is simply the value of the integrand at this point, times a constant estimating the integration along the rest of the curve. To prove it, we therefore have to show that the rest of the integration is bounded independently of $\varepsilon$. In particular, we need to show that the real part of the exponent is decreasing along the curve uniformly in $\varepsilon$, as we move away from the critical point. The computation is not difficult, because we have

$$
\partial_{w} F=\left(w-w_{+}\right)\left(w-w_{-}\right)\left(1-w^{2}\right)^{-1} .
$$

We get

$$
\partial_{r} \operatorname{Re}\left[\varepsilon^{-3 / 2} F\left(\varepsilon^{1 / 2}\left(\overline{\mathbf{w}}_{+}^{\varepsilon}+r e^{ \pm i \pi / 4}\right), \varepsilon^{1 / 2} \widetilde{\mathbf{x}}_{\varepsilon}, \varepsilon \tilde{u}_{\varepsilon}\right)\right]=-K r^{2},
$$

where

$$
K=\frac{\sqrt{2}\left(1-w^{2}+\tilde{r}^{2}+[4 w(w+x)+2 \sqrt{2} \tilde{r}(w+x)]\right)}{\left(w^{2}+\sqrt{2} \tilde{r} w-1\right)^{2}+2 \tilde{r}^{2} w^{2}+2 \sqrt{2} \tilde{r}^{3} w+\tilde{r}^{4}},
$$


with $w=\varepsilon^{1 / 2} \overline{\mathbf{w}}_{+}^{\varepsilon}, x=\varepsilon^{1 / 2} \widetilde{\mathbf{x}}_{\varepsilon}, u=\varepsilon \tilde{u}_{\varepsilon}$ and $\tilde{r}=\varepsilon^{1 / 2} r$. We will show that $K \geqslant \frac{1}{20} \sqrt{2}$. In the numerator, if $w \geqslant 0$, the term in square brackets is non-negative by assumption, so we can drop it to get a lower bound. In the denominator we can use

$$
\left(1-w^{2}+\sqrt{2} \tilde{r} w\right)^{2} \leqslant 2\left(1-w^{2}\right)^{2}+4 \tilde{r}^{2} w^{2} \quad \text { and } \quad \tilde{r}^{3} w \leqslant \frac{1}{2} \tilde{r}^{2}+\frac{1}{2} \tilde{r}^{4} w^{2},
$$

as well as $\tilde{r}^{4} \leqslant 2 \tilde{r}^{2}$ to bound it by

$$
10\left(\left(1-w^{2}\right)^{2}+\tilde{r}^{2} w^{2}+\tilde{r}^{2}\right)=20\left(\frac{1}{2}\left(1-w^{2}\right)\left(1-w^{2}-\tilde{r}^{2}\right)+\tilde{r}^{2}\right) .
$$

So, we just have to show that

$$
1-w^{2}+\tilde{r}^{2} \geqslant \frac{1}{2}\left(1-w^{2}\right)\left(1-w^{2}-\tilde{r}^{2}\right)+\tilde{r}^{2},
$$

which, since $w \in[0,1)$, is easily seen to be true. It is not hard to see that these inequalities remain true for $\overline{\mathbf{w}}_{+} \geqslant-\delta$ for some $\delta>0$.

Next, we check that the exponent is decreasing along the arc of $C_{\varepsilon}$ ending at $2 \varepsilon^{-1 / 2}$. Using now $\partial_{w} F=\left(w^{2}+2 x w-u\right)\left(1-w^{2}\right)^{-1}$, we have to show that

$$
\operatorname{Re}\left[\left(\left(e^{i \theta}+1\right)^{2}+2 \varepsilon^{1 / 2} \widetilde{\mathbf{x}}_{\varepsilon}\left(e^{i \theta}+1\right)-\varepsilon u\right)\left(1-\left(e^{i \theta}+1\right)^{2}\right)^{-1} i e^{i \theta}\right]>0
$$

for $\theta \in(0, \alpha \pi)$ for some $\alpha \leqslant \frac{1}{2}$. The real part is easily computed to be

$$
(5+4 \cos \theta)^{-1}\left(4(\cos \theta+1)+2 \varepsilon^{1 / 2} \widetilde{\mathbf{x}}_{\varepsilon}+\varepsilon \tilde{u}\right) \sin \theta,
$$

so we only need to show that $4(\cos \theta+1)+2 \varepsilon^{1 / 2} \widetilde{\mathbf{x}}_{\varepsilon}+\varepsilon \tilde{u}_{\varepsilon} \geqslant 0$ in this region. Now, $\cos \theta \geqslant 0$, so this is at least $4+2 \varepsilon^{1 / 2} \widetilde{\mathbf{x}}_{\varepsilon}+\varepsilon \tilde{u}_{\varepsilon}$. We have $\tilde{u}_{\varepsilon} \geqslant-\mathbf{x}_{\varepsilon}^{2}$, so the exponent is decreasing, as long as

$$
\widetilde{\mathbf{x}}_{\varepsilon} \in\left(\varepsilon^{-1 / 2}(1-\sqrt{5}), \varepsilon^{-1 / 2}(1+\sqrt{5})\right) .
$$

Proof of (iii). The situation now is a little different because we may not be able to move to the critical point without passing through a pole. On the other hand, we do not really need to because we are not trying to get an optimal estimate. Instead we deform the contour so that it passes through the real line at

$$
q:=\left(1+\left|\tilde{u}_{\varepsilon}\right|+\left|\widetilde{\mathbf{x}}_{\varepsilon}\right|\right)^{-1} \in\left(0, \varepsilon^{-1 / 2}\right),
$$

then move in the vertical direction until we hit the straight line from the proof of (ii) coming at angle $\frac{1}{4} \pi$ out of the critical point, and then continue until hitting the curve $C_{\varepsilon}$ as before. Along the vertical part, we have

$$
\partial_{r} \operatorname{Re}\left[\varepsilon^{-3 / 2} F\left(\varepsilon^{1 / 2}\left(q+i r, \varepsilon^{1 / 2} \widetilde{\mathbf{x}}_{\varepsilon}, \varepsilon \tilde{u}_{\varepsilon}\right)\right]=\left(-2\left(q+\widetilde{\mathbf{x}}_{\varepsilon}\right)+\mathcal{O}(\varepsilon)\right) r .\right.
$$


Since $\widetilde{\mathbf{x}}_{\varepsilon} \geqslant 0$, the real part is decreasing along this verticle piece. Along the straight piece at angle $\frac{1}{4} \pi$, the proof of (ii) still works to prove the uniform decrease. The proof of uniform decrease along the arc of $C_{\varepsilon}$ is different for $F_{\varepsilon}$ and $\bar{F}_{\varepsilon}$, and depends on the precise dependence of $u_{\varepsilon}, \mathbf{x}_{\varepsilon}, \bar{u}_{\varepsilon}$ and $\overline{\mathbf{x}}_{\varepsilon}$ on the bare variables $u$ and $\mathbf{x}$. In the first case,

$$
\begin{aligned}
& \operatorname{Re}\left[\varepsilon^{-3 / 2} F\left(\varepsilon^{1 / 2} w, \varepsilon^{1 / 2} \mathbf{x}_{\varepsilon}, \varepsilon u_{\varepsilon}\right)\right] \\
& \quad=\operatorname{Re}\left[\varepsilon^{-3 / 2}\left(\operatorname{arctanh} \varepsilon^{1 / 2} w-\varepsilon^{1 / 2} w\right)\right]-\varepsilon^{-1} \mathbf{x} \operatorname{Re}\left[\log \left(1+\varepsilon^{1 / 2} w\right)\right]
\end{aligned}
$$

does not even depend on $u$, and decreases uniformly along the arc of $C_{\varepsilon}$ as long as $\mathbf{x} \geqslant 0$. In the second case,

$$
\begin{aligned}
& \operatorname{Re}\left[\varepsilon^{-3 / 2} F\left(\varepsilon^{1 / 2} w, \varepsilon^{1 / 2} \overline{\mathbf{x}}_{\varepsilon}, \varepsilon \bar{u}_{\varepsilon}\right)\right] \\
& \quad=\operatorname{Re}\left[\varepsilon^{-3 / 2}\left(\operatorname{arctanh} \varepsilon^{1 / 2} w-\varepsilon^{1 / 2} w\right)\right]-\varepsilon^{-1}\left(\mathbf{x}+\varepsilon^{1 / 2} u\right) \operatorname{Re}\left[\log \left(1+\varepsilon^{1 / 2} w\right)\right],
\end{aligned}
$$

so we require $u>-\varepsilon^{-1 / 2} \mathbf{x}$. Now that we have checked that the exponent is decreasing uniformly along the curve, we end up with an estimate in terms of the value of the integrands in (B.1) and (B.2) at $\widetilde{w}_{i}=\left(1+\left|\tilde{u}_{\varepsilon}\right|+\left|\widetilde{\mathbf{x}}_{\varepsilon}\right|\right)^{-1}, i=1,2$. The exponent is bounded and we pick up a term $\log \left(2+\left|\widetilde{\mathbf{x}}_{\varepsilon}\right|+\left|\tilde{u}_{\varepsilon}\right|\right)$ from the denominator.

Proof of (iv) and (v). The critical points are complex now, and we deform $C_{\varepsilon}$ to a contour passing through both $\mathbf{w}_{+}^{\varepsilon}$ and $\mathbf{w}_{-}^{\varepsilon}$. The contour consists of a straight line from zero to $\mathbf{w}_{+}^{\varepsilon}$, then a straight line moving out from $\mathbf{w}_{+}^{\varepsilon}$ at angle $\frac{1}{4} \pi$ until it hits $C_{\varepsilon}$, and then it continues along $C_{\varepsilon}$ in the usual clockwise direction until it hits the real axis, after which it follows the reflected curve across the real axis, back to zero. However, to avoid the singularity at zero from the denominator in (B.1) and (B.2), we cut off the tip of the curve just to the right of zero.

The first thing we need to check is that the real part of the exponent is increasing uniformly in $\varepsilon$ along the linear piece between zero and $\overline{\mathbf{w}}_{+}^{\varepsilon}$. Using

$$
\partial_{w} F=\left(w-w_{+}\right)\left(w-w_{-}\right)\left(1-w^{2}\right)^{-1},
$$

we compute

$$
\partial_{r} \varepsilon^{-3 / 2} F\left(\varepsilon^{1 / 2} r \overline{\mathbf{w}}_{+}^{\varepsilon}, \varepsilon^{1 / 2} \widetilde{\mathbf{x}}_{\varepsilon}, \varepsilon \tilde{u}_{\varepsilon}\right)=(r-1)\left(\overline{\mathbf{w}}_{+}^{\varepsilon}\right)^{2}\left(r \overline{\mathbf{w}}_{+}^{\varepsilon}-\overline{\mathbf{w}}_{-}^{\varepsilon}\right) v,
$$

where $v=\left(1-\varepsilon r^{2}\left(\overline{\mathbf{w}}_{+}^{\varepsilon}\right)^{2}\right)^{-1}$. Here, $r \in(0,1)$ so $r-1<0$. From the assumptions $\overline{\mathbf{w}}_{ \pm}^{\varepsilon}=R e^{ \pm i \theta}$ with $R>0$ and $\theta \in\left(0, \frac{1}{2} \pi\right)$, so

$$
\left(\overline{\mathbf{w}}_{+}^{\varepsilon}\right)^{2}\left(r \overline{\mathbf{w}}_{+}^{\varepsilon}-\overline{\mathbf{w}}_{-}^{\varepsilon}\right)=R^{3}\left(r e^{3 i \theta}-e^{-i \theta}\right)=R^{\prime} e^{i \theta^{\prime}},
$$


with $R^{\prime}>0$ and $\theta^{\prime} \in\left(\frac{1}{2} \pi, \frac{3}{2} \pi\right)$. Now, since $\left|\overline{\mathbf{w}}_{+}^{\varepsilon}\right| \leqslant C \varepsilon^{-1 / 4}$, for $\varepsilon$ small enough we have $\theta^{\prime}+\arg v \in\left(\frac{1}{2} \pi, \frac{3}{2} \pi\right)$, and hence $\operatorname{Re}\left[\left(\overline{\mathbf{w}}_{+}^{\varepsilon}\right)^{2}\left(r \overline{\mathbf{w}}_{+}^{\varepsilon}-\overline{\mathbf{w}}_{-}^{\varepsilon}\right) v\right]$ is strictly positive, uniformly in $\varepsilon \in\left[0, \varepsilon_{0}\right]$. Note this argument is not uniform in $\theta^{\prime}$ and hence we end up with a constant which blows up with $\left|\tilde{u}_{\varepsilon}\right|^{1 / 2} /\left|\widetilde{\mathbf{x}}_{\varepsilon}\right|$.

Next, we have to check that the real part of the exponent is decreasing uniformly in $\varepsilon$ along the line at angle $\frac{1}{4} \pi$ coming out of $\overline{\mathbf{w}}_{+}^{\varepsilon}$, so that this piece of the integral is bounded uniformly in $\varepsilon$. Using the formula for $\partial_{w} F$ above,

$$
\begin{aligned}
\partial_{r} \varepsilon^{-3 / 2} F\left(\varepsilon^{1 / 2}\left(\overline{\mathbf{w}}_{+}^{\varepsilon}+r e^{i \pi / 4}\right), \varepsilon^{1 / 2} \widetilde{\mathbf{x}}_{\varepsilon}, \varepsilon \tilde{u}_{\varepsilon}\right) & =\left.\varepsilon^{-1} \partial_{w} F e^{i \pi / 4}\right|_{w=\varepsilon^{1 / 2}\left(\overline{\mathbf{w}}_{+}^{\varepsilon}+r e^{i \pi / 4}\right)} \\
& =-r\left(2\left|\tilde{\mathbf{y}}_{\varepsilon}\right|^{1 / 2}-r e^{i 3 \pi / 4}\right) v
\end{aligned}
$$

where $\tilde{\mathbf{y}}_{\varepsilon}=\widetilde{\mathbf{x}}_{\varepsilon}^{2}+\tilde{u}_{\varepsilon}$ and $v=\left(1-\varepsilon\left(\mathbf{w}_{+}^{\varepsilon}+r e^{i \pi / 4}\right)^{2}\right)^{-1}$. Hence, the real part is less than or $-C r\left|\mathbf{y}_{\varepsilon}\right|^{1 / 2}$ as long as $\arg v \in\left(-\frac{1}{4} \pi, \frac{1}{2} \pi\right)$, i.e. it is enough that

$$
\arg \left(1-\varepsilon\left(\mathbf{w}_{+}^{\varepsilon}+r e^{i \pi / 4}\right)^{2}\right) \in\left(-\frac{1}{2} \pi, \frac{1}{4} \pi\right),
$$

which is true for small enough $\varepsilon$ since $r$ is less than $\sqrt{2} \varepsilon^{-1 / 2}$, and $\left|\mathbf{w}_{+}^{\varepsilon}\right| \leqslant C \varepsilon^{-1 / 4}$. Finally, we need to check that the exponent is still decreasing as we move along the arc of the curve $C_{\varepsilon}$, but the proof given in case (ii) above works in the same way here.

Hence, we have an estimate $F_{\varepsilon}(\mathbf{x}, u) \leqslant \widehat{F}_{\varepsilon}\left(\mathbf{x}_{\varepsilon}, u_{\varepsilon}\right)$.

To prove (iv) we need to estimate $\widehat{F}_{\varepsilon}\left(\mathbf{x}_{\varepsilon}, u_{\varepsilon}\right)$. We use (B.3). First of all, since $\left|\mathbf{w}_{+}^{\varepsilon}\right| \leqslant C \varepsilon^{-1 / 4}$, one has $\left|\nu_{i}\left(\varepsilon^{1 / 2} \mathbf{w}_{+}^{\varepsilon}\right)\right| \leqslant C \varepsilon^{1 / 2}$. Furthermore,

$$
\left|\mathbf{x y}-\frac{1}{3} \mathbf{x}^{3}-\frac{2}{3} \mathbf{y}^{3 / 2}\right|,\left|\mathbf{x y}-\frac{1}{2} \mathbf{x}^{2} \sqrt{\mathbf{y}}-\frac{1}{2} \mathbf{y}^{3 / 2}\right| \leqslant C\left(|\mathbf{x}|^{3}+|u|^{3 / 2}\right) .
$$

Thus,

$$
\begin{aligned}
\operatorname{Re}\left[\nu_{1}\left(\varepsilon^{1 / 2} \mathbf{w}_{+}\right)\left(\mathbf{x y}-\frac{1}{3} \mathbf{x}^{3}-\frac{2}{3} \mathbf{y}^{3 / 2}\right)+\nu_{2}\left(\varepsilon^{1 / 2} \mathbf{w}_{+}\right)\left(\mathbf{x y}-\frac{1}{2} \mathbf{x}^{2} \sqrt{\mathbf{y}}-\frac{1}{2} \mathbf{y}^{3 / 2}\right)\right] \\
\leqslant C \varepsilon^{1 / 2}\left(|\mathbf{x}|^{3}+|u|^{3 / 2}\right) .
\end{aligned}
$$

Here, $\mathbf{x}=\mathbf{x}_{\varepsilon}$ and $\mathbf{y}=\mathbf{x}_{\varepsilon}^{2}+u_{\varepsilon}$. So, it just remains to bound the real part of the term $\mathbf{x y}-\frac{1}{3} \mathbf{x}^{3}-\frac{2}{3} \mathbf{y}^{3 / 2}$. Since $\mathbf{y}<0$, the real part of the third term vanishes. Write the first and second as

$$
\mathbf{x}_{\varepsilon} u_{\varepsilon}+\frac{2}{3} \mathbf{x}_{\varepsilon}^{3}=\mathbf{x}_{\varepsilon} u_{\varepsilon}-\frac{2}{3}\left|\mathbf{x}_{\varepsilon}\right|^{3} \leqslant-\left(\frac{2}{3}-\delta\right)|\mathbf{x}|^{3}+C \delta^{-1}|u|^{3 / 2},
$$

which yields the desired estimate.

To prove (v), it remains to show that $\widehat{F}_{\varepsilon}\left(\overline{\mathbf{x}}_{\varepsilon}, \bar{u}_{\varepsilon}\right) \leqslant C$. By the same argument as above, we have

$$
\widehat{F}_{\varepsilon}\left(\overline{\mathbf{x}}_{\varepsilon}, \bar{u}_{\varepsilon}\right) \leqslant\left(1+C \varepsilon^{1 / 2}\right)\left(\left|\mathbf{x}_{\varepsilon}\right|^{3}+\left|u_{\varepsilon}\right|^{3 / 2}\right),
$$

which proves it. 
Proof of Proposition B.1. The first step is to obtain a bound when $\mathbf{x}_{1}, \mathbf{x}_{2} \geqslant 2$ and the cutoff is at $L=0$. In this case, $\left(\mathfrak{g}_{L}^{\varepsilon}\right)^{L,-}$ becomes simply $\left(\mathfrak{g}^{\varepsilon}\right)^{-}$, and the operator appearing in the result is given by

$$
\begin{aligned}
& \left(\mathbf{S}_{-1, \mathbf{x}_{1}}^{\varepsilon}\right)^{*} \overline{\mathbf{S}}_{-1,-\mathbf{x}_{2}}^{\varepsilon, \operatorname{epi}\left(\left(\mathfrak{g}^{\varepsilon}\right)^{-}\right)}\left(z_{1}, z_{2}\right) \\
& \quad=\int_{\mathbf{b}, z \in \mathbb{R}, \mathbf{s} \in[0, \varepsilon n)} d z \mathbb{P}_{\mathbf{B}_{\varepsilon}(0)=z}\left(\boldsymbol{\tau}_{\varepsilon} \in d \mathbf{s}, \mathbf{B}_{\varepsilon}\left(\boldsymbol{\tau}_{\varepsilon}\right) \in d \mathbf{b}\right) \mathbf{S}_{-1, \mathbf{x}_{1}}^{\varepsilon}\left(z, z_{1}\right) \overline{\mathbf{S}}_{-1,-\mathbf{x}_{2}-\mathbf{s}}^{\varepsilon}\left(\mathbf{b}, z_{2}\right)
\end{aligned}
$$

(here $n=\frac{1}{2} \varepsilon^{-3 / 2}+\varepsilon^{-1} \mathbf{x}_{2}+1$ from (3.8)). We think of the right-hand side as an integral of operator kernels in $z_{1}$ and $z_{2}$ over some extra parameters $z, \mathbf{b}$ and $\mathbf{s}$. We estimate exactly as in (A.7)-(A.9) to see that $\left\|\left(\mathbf{S}_{-1, \mathbf{x}_{1}}^{\varepsilon}\right) * \overline{\mathbf{S}}_{-1,-\mathbf{x}_{2}}^{\varepsilon, \text { epi }\left(\left(\mathfrak{g}^{\varepsilon}\right)^{-}\right)}\right\|_{1}$ is bounded by

$$
\int_{\mathbf{b}, z \in \mathbb{R}, \mathbf{s} \in[0, \varepsilon n)} d z \mathbb{P}_{\mathbf{B}_{\varepsilon}(0)=z}\left(\boldsymbol{\tau}_{\varepsilon} \in d \mathbf{s}, \mathbf{B}_{\varepsilon}\left(\boldsymbol{\tau}_{\varepsilon}\right) \in d \mathbf{b}\right) \exp \left\{F_{\varepsilon}\left(\mathbf{x}_{1}, z\right)+\bar{F}_{\varepsilon}\left(-\mathbf{x}_{2}-\mathbf{s}, \mathbf{b}\right)\right\} .
$$

It is convenient to recall at this point that, in the context of the above bound, the parameters appearing in (B.1), (B.2) and Lemma B.2 are given by

$$
\begin{array}{ll}
\mathbf{x}_{\varepsilon}=\mathbf{x}_{1}-\frac{1}{2} \varepsilon^{1 / 2} z-\frac{1}{2} \varepsilon, & u_{\varepsilon}=z-\varepsilon^{1 / 2}, \\
\overline{\mathbf{x}}_{\varepsilon}=-\mathbf{x}_{2}-\mathbf{s}+\frac{1}{2} \varepsilon^{1 / 2} \mathbf{b}+\frac{3}{2} \varepsilon, & \bar{u}_{\varepsilon}=\mathbf{b}+\varepsilon^{1 / 2} .
\end{array}
$$

We remark that $\mathbf{x}_{1}$ and $\mathbf{x}_{2}$ here are fixed; constants in the estimates below may (and will) depend on them.

Consider first the case $z \geqslant-1$. Recalling that $\mathbf{x}_{2} \geqslant 2$, one can check that $\mathbf{x}_{\varepsilon}^{2}+u_{\varepsilon} \geqslant 0$ and that, if we let $\bar{z}=\frac{1}{2} \varepsilon^{-1}+\varepsilon^{-1} \mathbf{x}_{1}$ then $-\mathbf{x}_{\varepsilon}+\sqrt{\mathbf{x}_{\varepsilon}^{2}+u_{\varepsilon}}-\varepsilon^{-1 / 2}$ is negative for $z \in[-1, \bar{z})$ and non-negative for $z \geqslant \bar{z}$. In the first case, we may use Lemma B.2 (ii) and the fact that for $\mathbf{y}=\mathbf{x}_{\varepsilon}^{2}+u_{\varepsilon} \geqslant 0$ we have that $\nu_{1}$ and $\nu_{2}$ are positive and bounded and

$$
\mathbf{x y}-\frac{1}{2} \mathbf{x}^{2} \sqrt{\mathbf{y}}-\frac{1}{2} \mathbf{y}^{3 / 2} \leqslant 0
$$

to find a $C<\infty$ such that $\widehat{F}_{\varepsilon}\left(\mathbf{x}_{\varepsilon}, z\right) \leqslant C-\frac{1}{3}|z|^{3 / 2}$, while for $z \geqslant \bar{z}$ then we may simply use Lemma B.2(i) to get a much better bound. On the other hand, when $z<-1$, we use Lemma B.2 (iii) to find $C<\infty$ such that $F_{\varepsilon}\left(\mathbf{x}_{1}, z\right) \leqslant C(1+\log |z|)$. Therefore, we may choose a constant $C>0$ depending on $\overline{\boldsymbol{\alpha}}$ such that

$$
F_{\varepsilon}\left(\mathbf{x}_{1}, z\right) \leqslant C+C \log |z| \mathbf{1}_{z \leqslant-\overline{\boldsymbol{\alpha}}}-\frac{1}{3}|z|^{3 / 2} \mathbf{1}_{z \geqslant-\overline{\boldsymbol{\alpha}}} .
$$

Note that we got a better bound than (A.12), because we are assuming $\mathbf{x}_{1} \geqslant 2$.

Next, we deal with the other term inside the exponential in (B.4). Assume first that $z \leqslant \mathfrak{g}(0)$ (so in particular what follows holds also for $z \leqslant-\overline{\boldsymbol{\alpha}}$ ). We claim then that, as in (A.11), there are $\kappa_{1}>0$ and $C<\infty$ such that

$$
\bar{F}_{\varepsilon}\left(-\mathbf{x}_{2}-\mathbf{s}, \mathbf{b}\right) \leqslant-\kappa_{1} \mathbf{s}^{3}+C .
$$


We still have the linear lower bound (A.10), $\mathbf{b} \geqslant-\overline{\boldsymbol{\alpha}}-\bar{\gamma} \mathbf{s}$. Note, first of all, that the random walk simply cannot jump upwards farther than $\varepsilon^{-1 / 2} \mathbf{s}$ in time $\mathbf{s}$, and therefore $\mathbf{b} \leqslant z+\varepsilon^{-1 / 2} \mathbf{s}$. Then, we have $\overline{\mathbf{x}}_{\varepsilon} \leqslant 0$ for small enough $\varepsilon$ (see (B.5); here we have used $z \leqslant \mathfrak{g}(0)$ ). Assume furthermore that $\overline{\mathbf{x}}_{\varepsilon}^{2}+\mathbf{b} \geqslant 0$. It is easy to check then that, for $\mathbf{s}<\varepsilon n$ (as we have in (B.4)), the hypotheses of Lemma B.2 (ii) are satisfied for small $\varepsilon$, so we have using also (B.3) that

$$
\bar{F}_{\varepsilon}\left(-\mathbf{x}_{2}-\mathbf{s}, \mathbf{b}\right) \leqslant \widehat{F}_{\varepsilon}\left(\overline{\mathbf{x}}_{\varepsilon}, \bar{u}_{\varepsilon}\right) \leqslant\left(1+\nu_{1}\right)\left(\overline{\mathbf{x}}_{\varepsilon} \mathbf{b}+\frac{2}{3} \overline{\mathbf{x}}_{\varepsilon}^{3}-\frac{2}{3}\left(\overline{\mathbf{x}}_{\varepsilon}^{2}+\mathbf{b}\right)^{3 / 2}\right),
$$

with $\nu_{1}$ non-negative and bounded (note that the second term in (B.3) is clearly negative in our case; additionaly, note that in (B.3) the middle term in the first parenthesis has $\mathrm{a}+\frac{1}{3}$ in the middle term, the $-\frac{2}{3}$ here is because we are writing $\left.\mathbf{x y}-\frac{1}{3} \mathbf{x}^{3}=\mathbf{x u}+\frac{2}{3} \mathbf{x}^{3}\right)$. Using the linear lower bound $\mathbf{b} \geqslant-\widetilde{\boldsymbol{\alpha}}-\widetilde{\gamma} \mathbf{s}$, and the definition (B.5) of $\overline{\mathbf{x}}_{\varepsilon}$, which in our case is negative, (B.6) follows from (B.7). The alternative $\overline{\mathbf{x}}_{\varepsilon}^{2}+\mathbf{b}<0$ can only happen for $\mathbf{b}<0$ and $0 \leqslant \mathbf{s} \leqslant \mathbf{s}_{0}$, for a constant $\mathbf{s}_{0}<\infty$ depending on $\overline{\boldsymbol{\alpha}}, \overline{\boldsymbol{\gamma}}$ and $\mathbf{x}_{2}$. Then, since we also have $\mathbf{b} \geqslant-\overline{\boldsymbol{\alpha}}-\overline{\boldsymbol{\gamma}} \mathbf{s}_{0}$, we can use Lemma B.2(v) to get $\widehat{F}_{\varepsilon}\left(-\mathbf{x}_{2, \varepsilon}-\mathbf{s}, \mathbf{b}\right) \leqslant C$, and hence, since $\mathbf{s} \leqslant \mathbf{s}_{0},($ B.6).

Now suppose $z>\mathfrak{g}(0)$. We can use Lemma B.2(ii) because the condition

$$
-\overline{\mathbf{x}}_{\varepsilon}+\sqrt{\overline{\mathbf{x}}_{\varepsilon}^{2}+\bar{u}_{\varepsilon}}<\varepsilon^{-1 / 2}
$$

reduces in this case to $\frac{1}{2} \varepsilon^{-3 / 2}-\varepsilon^{-1} s+\varepsilon^{-1} \mathbf{x}_{2}>-1$, which holds because $s<\varepsilon n=\frac{1}{2} \varepsilon^{-1 / 2}$. We get $\bar{F}_{\varepsilon}\left(-\mathbf{x}_{2}, z\right) \leqslant \widehat{F}_{\varepsilon}\left(\overline{\mathbf{x}}_{\varepsilon}, \bar{u}_{\varepsilon}\right) \leqslant C$.

Let $\boldsymbol{\sigma}^{\varepsilon}$ be the hitting time of the epigraph of $-\overline{\boldsymbol{\alpha}}-\bar{\gamma}|\mathbf{x}|$ by the random walk $\mathbf{B}_{\varepsilon}$. Clearly, $\boldsymbol{\tau}^{\varepsilon} \geqslant \boldsymbol{\sigma}^{\varepsilon}$. We have

$$
\mathbb{P}_{z}\left(\boldsymbol{\sigma}^{\varepsilon} \leqslant \mathbf{s}\right)=\mathbb{P}_{z}\left(\sup _{0 \leqslant \mathbf{x} \leqslant \mathbf{s}} \mathbf{B}_{\varepsilon}(\mathbf{x})+\overline{\boldsymbol{\alpha}}+\bar{\gamma} \mathbf{x}>0\right)
$$

Note that $\mathbf{B}_{\varepsilon}(\mathbf{x})+\overline{\boldsymbol{\alpha}}+\bar{\gamma} \mathbf{x}$ is a submartingale, and by Doob's submartingale inequality, for any $\lambda>0$,

$$
\mathbb{P}_{z}\left(\sup _{0 \leqslant \mathbf{x} \leqslant \mathbf{s}} \mathbf{B}_{\varepsilon}(\mathbf{x})+\overline{\boldsymbol{\alpha}}+\bar{\gamma} \mathbf{x}>0\right) \leqslant \mathbb{E}_{z}\left[e^{\lambda\left(\mathbf{B}_{\varepsilon}(\mathbf{s})+\overline{\boldsymbol{\alpha}}+\overline{\boldsymbol{\gamma}} \mathbf{s}\right)}\right]=e^{\lambda(z+\overline{\boldsymbol{\alpha}}+\overline{\boldsymbol{\gamma}} \mathbf{s})+\varepsilon^{-1} \mathbf{s} \log M\left(\varepsilon^{1 / 2} \lambda\right)},
$$

where $M(\lambda)=e^{\lambda} /\left(2-e^{-\lambda}\right), \lambda>-\log 2$, is the moment generating function of a centered negative Geom $\left[\frac{1}{2}\right]$ random variable. By choosing $\lambda>0$ carefully, we can find a $\kappa_{2}>0$ such that the right-hand side is bounded above by $\exp \left\{-\kappa_{2} \mathbf{s}^{-1}(z+\overline{\boldsymbol{\alpha}}+\bar{\gamma} \mathbf{s})^{2}\right\}$ when $z \leqslant-\overline{\boldsymbol{\alpha}}$, and therefore, for such $z$,

$$
\mathbb{P}_{z}\left(\boldsymbol{\tau}_{\varepsilon} \leqslant \mathbf{s}\right) \leqslant \exp \left\{-\kappa_{2} \mathbf{s}^{-1}(z+\overline{\boldsymbol{\alpha}}+\bar{\gamma} \mathbf{s})^{2}\right\}
$$


Putting it all together, we have that $\left\|\left(\mathbf{S}_{-1, \mathbf{x}_{1}}^{\varepsilon}\right) * \overline{\mathbf{S}}_{-1,-\mathbf{x}_{2}}^{\varepsilon, \text { epi }\left(\left(\mathfrak{g}^{\varepsilon}\right)^{-}\right)}\right\|_{1}$ is bounded by a constant multiple of

$$
\begin{aligned}
\int_{-\infty}^{-\overline{\boldsymbol{\alpha}}} d z & \int_{0}^{\infty} d s e^{-\kappa_{2} \mathbf{s}^{-1}(z+\overline{\boldsymbol{\alpha}}+\overline{\boldsymbol{\gamma}} \mathbf{s})^{2}+C \log |z|-\kappa_{1} \mathbf{s}^{3}} \\
& \quad+\int_{-\overline{\boldsymbol{\alpha}}}^{\infty} d z \int_{\mathbf{s} \geqslant 0, \mathbf{b} \in \mathbb{R}} \mathbb{P}_{\mathbf{B}_{\varepsilon}(0)=z}\left(\boldsymbol{\tau}_{\varepsilon} \in d \mathbf{s}, \mathbf{B}_{\varepsilon}\left(\boldsymbol{\tau}_{\varepsilon}\right) \in d \mathbf{b}\right) e^{-|z|^{3 / 2} / 3+\bar{F}_{\varepsilon}\left(-\mathbf{x}_{2}-\mathbf{s}, \mathbf{b}\right)}
\end{aligned}
$$

The first integral clearly converges. The second one can be split into $z \in[-\overline{\boldsymbol{\alpha}}, \mathfrak{g}(0)]$ and $(\mathfrak{g}(0), \infty)$. On the first piece the bound (B.6) still holds, so the integral is clearly finite. On the second piece we observe that $z>\mathfrak{g}(0)$ forces $\boldsymbol{\tau}_{\varepsilon}=0$ and $\mathbf{b}=z$, so the integral is just

$$
\int_{-\overline{\boldsymbol{\alpha}}}^{\infty} d z e^{-|z|^{3 / 2} / 3+\bar{F}_{\varepsilon}\left(-\mathbf{x}_{2}, z\right)} \leqslant C
$$

The next step is to extend to $L>0$.

Suppose that $\mathfrak{f}_{\ell}$ is the LC cutoff of some $\mathfrak{f} \in \mathrm{LC}$ to the right of position $\ell$. Then if $L>\ell$, because the random walk is free for time $L-\ell$ (see (2.35)),

$$
\left(\mathbf{S}_{-1, \mathbf{x}_{1}-L}^{\varepsilon}\right)^{*} \overline{\mathbf{S}}_{-1,-\mathbf{x}_{2}+L}^{\varepsilon, \operatorname{epi}\left(\left(\mathfrak{f}_{\ell}\right)^{L,-}\right)}=\left(\mathbf{S}_{-1, \mathbf{x}_{1}-\ell}^{\varepsilon}\right)^{*} \overline{\mathbf{S}}_{-1,-\mathbf{x}_{2}+\ell}^{\varepsilon, \operatorname{epi}\left(\mathfrak{f}^{\ell,-}\right)}
$$

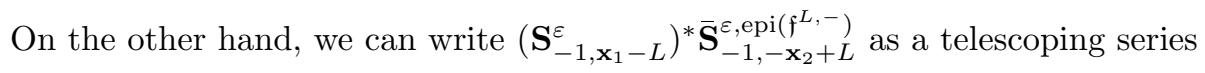

$$
\left(\mathbf{S}_{-1, \mathbf{x}_{1}}^{\varepsilon}\right)^{*} \overline{\mathbf{S}}_{-1,-\mathbf{x}_{2}}^{\varepsilon, \operatorname{epi}\left(\mathfrak{f}^{0,-}\right)}+\sum_{\ell=1}^{L}\left[\left(\mathbf{S}_{-1, \mathbf{x}_{1}-\ell}^{\varepsilon}\right)^{*} \overline{\mathbf{S}}_{-1,-\mathbf{x}_{2}+\ell}^{\varepsilon, \operatorname{epi}\left(\mathfrak{f}^{\ell,-}\right)}-\left(\mathbf{S}_{-1, \mathbf{x}_{1}-(\ell-1)}^{\varepsilon}\right)^{*} \overline{\mathbf{S}}_{-1,-\mathbf{x}_{2}+\ell-1}^{\varepsilon, \operatorname{epi}\left(\mathfrak{f}^{\ell-1,-}\right)}\right]
$$

We have the following estimate.

Lemma B.3. For each $\delta>0$, there is a $C<\infty$ such that, for all $\ell \leqslant L$,

$$
\left\|\left(\mathbf{S}_{-1, \mathbf{x}_{1}-\ell}^{\varepsilon}\right)^{*} \overline{\mathbf{S}}_{-1,-\mathbf{x}_{2}+\ell}^{\varepsilon, \operatorname{epi}\left(\left(\mathfrak{g}_{L}^{\varepsilon}\right)^{\ell,-}\right)}-\left(\mathbf{S}_{-1, \mathbf{x}_{1}-(\ell-1)}^{\varepsilon}\right)^{*} \overline{\mathbf{S}}_{-1,-\mathbf{x}_{2}+\ell-1}^{\varepsilon, \operatorname{epi}\left(\left(\mathfrak{g}_{L}^{\varepsilon}\right)^{\ell-1,-}\right)}\right\|_{1} \leqslant C e^{-(2 / 3-\delta) \ell^{3}}
$$

Before proving the lemma we will employ it to finish the proof of Proposition B.1. Using (B.11) in (B.10) we have, for $\mathbf{x}_{1} \geqslant 2$ and $\mathbf{x}_{2} \geqslant 2$,

$$
\left\|\bar{\chi}_{0}\left(\mathbf{S}_{-1, \mathbf{x}_{1}-L}^{\varepsilon}\right)^{*} \overline{\mathbf{S}}_{-1,-\mathbf{x}_{2}+L}^{\left.\varepsilon, \operatorname{epi}\left(\mathfrak{g}_{L}^{\varepsilon}\right)^{L,-}\right)} \bar{\chi}_{0}\right\|_{1} \leqslant\left\|\bar{\chi}_{0}\left(\mathbf{S}_{-1, \mathbf{x}_{1}}^{\varepsilon}\right)^{*} \overline{\mathbf{S}}_{-1,-\mathbf{x}_{2}}^{\varepsilon, \operatorname{epi}\left(\left(\mathfrak{g}_{L}^{\varepsilon}\right)^{0,-}\right)} \bar{\chi}_{0}\right\|_{1}+C \sum_{\ell=1}^{L} e^{-\ell^{3} / 2} \leqslant C^{\prime}
$$

uniformly in $L$ as needed, using the $L=0$ case of the proposition (note that $\left(\mathfrak{g}_{L}^{\varepsilon}\right)^{0,-}(\mathbf{y})=$ $\left(\mathfrak{g}_{0}^{\varepsilon}\right)^{0,-}(\mathbf{y})$ for all $\left.\mathbf{y} \geqslant 0\right)$. The condition $\mathbf{x}_{1}, \mathbf{x}_{2} \geqslant 2$ can now clearly be dropped using this estimate by taking $L$ larger if needed and shifting $\mathfrak{g}$ slightly. 
Proof of Lemma B.3. Note first that, for all $\ell \leqslant L$, we have $\left(\mathfrak{g}_{L}^{\varepsilon}\right)^{\ell,-}(\mathbf{y})=\left(\mathfrak{g}_{\ell}^{\varepsilon}\right)^{\ell,-}(\mathbf{y})$ for all $\mathbf{y} \geqslant 0$. Using this and (B.9), we have that the operator inside the trace norm in (B.11) can be written as

$$
\left(\mathbf{S}_{-1, \mathbf{x}_{1}-\ell}^{\varepsilon}\right)^{*}\left[\overline{\mathbf{S}}_{-1,-\mathbf{x}_{2}+\ell}^{\varepsilon, e p i\left(\left(\mathfrak{g}_{\ell}^{\varepsilon}\right)^{\ell,-}\right)}-\overline{\mathbf{S}}_{-1,-\mathbf{x}_{2}+\ell}^{\varepsilon, e p i\left(\left(\mathfrak{g}_{\ell-1}^{\varepsilon}\right)^{\ell,-}\right)}\right]
$$

We can write the kernel of the operator in brackets at $\left(z, z_{2}\right)$ as

$$
\mathbb{E}_{B_{0}^{\varepsilon}=z}\left[\overline{\mathbf{S}}_{-1,-\mathbf{x}_{2}+\ell-\boldsymbol{\tau}_{\varepsilon}}\left(\mathbf{B}_{\varepsilon}\left(\boldsymbol{\tau}_{\varepsilon}\right)-z_{2}\right) \mathbf{1}_{\boldsymbol{\tau}_{\varepsilon}<\varepsilon n+\ell}-\overline{\mathbf{S}}_{-1,-\mathbf{x}_{2}+\ell-\overline{\boldsymbol{\tau}}_{\varepsilon}}\left(\mathbf{B}_{\varepsilon}\left(\overline{\boldsymbol{\tau}}_{\varepsilon}\right)-z_{2}\right) \mathbf{1}_{\overline{\boldsymbol{\tau}}_{\varepsilon}<\varepsilon n+\ell}\right],
$$

where $n$ is still $\frac{1}{2} \varepsilon^{-3 / 2}+\varepsilon^{-1} \mathbf{x}_{2}+1$ (as above) while $\boldsymbol{\tau}_{\varepsilon}$ is the hitting time of epi $\left(\left(\mathfrak{g}_{\ell}^{\varepsilon}\right)^{\ell,-}\right.$ ), and $\overline{\boldsymbol{\tau}}_{\varepsilon}$ is the hitting time of epi $\left(\left(\mathfrak{g}_{\ell-1}^{\varepsilon}\right)^{\ell,-}\right)$ by the scaled walk

$$
\mathbf{B}_{\varepsilon}(\mathbf{x})=\varepsilon^{1 / 2}\left(B_{\varepsilon^{-1} \mathbf{x}}+2 \varepsilon^{-1} \mathbf{x}-1\right)
$$

Note that $\overline{\boldsymbol{\tau}}_{\varepsilon} \geqslant \boldsymbol{\tau}_{\varepsilon}$ with equality whenever $\boldsymbol{\tau}_{\varepsilon} \geqslant 1$. So, we can put $\mathbf{1}_{\boldsymbol{\tau}_{\varepsilon}<1}$ inside the expectation and use the sum instead of the difference in our bounds. We can then estimate the trace norm of $(\mathrm{B} .12)$ (on $\left.L^{2}((-\infty, 0])\right)$ by

$$
\begin{aligned}
& \int p_{z}^{\varepsilon}(d \mathbf{s}, d \overline{\mathbf{s}}, d \mathbf{b}, d \overline{\mathbf{b}}) d z \mathbf{1}_{\mathbf{s}<1}\left\|\mathbf{S}_{-1, \mathbf{x}_{1}-\ell}^{\varepsilon}\left(z, z_{1}\right) \mathbf{1}_{z_{1} \leqslant 0} \overline{\mathbf{S}}_{-1,-\mathbf{x}_{2}+\ell-\mathbf{s}}^{\varepsilon}\left(\mathbf{b}-z_{2}\right) \mathbf{1}_{z_{2} \leqslant 0}\right\|_{1} \\
& +\int p_{z}^{\varepsilon}(d \mathbf{s}, d \overline{\mathbf{s}}, d \mathbf{b}, d \overline{\mathbf{b}}) d z \mathbf{1}_{\mathbf{s}<1, \overline{\mathbf{s}}<\varepsilon n+\ell}\left\|\mathbf{S}_{-1, \mathbf{x}_{1}-\ell}^{\varepsilon}\left(z, z_{1}\right) \mathbf{1}_{z_{1} \leqslant 0} \overline{\mathbf{S}}_{-1,-\mathbf{x}_{2}+\ell-\overline{\mathbf{s}}}^{\varepsilon}\left(\overline{\mathbf{b}}-z_{2}\right) \mathbf{1}_{z_{2} \leqslant 0}\right\|_{1},
\end{aligned}
$$

where the trace norms are as operator kernels in $z_{1}$ and $z_{2}$ (with $z$ fixed), and

$$
p_{z}^{\varepsilon}(d \mathbf{s}, d \overline{\mathbf{s}}, d \mathbf{b}, d \overline{\mathbf{b}})
$$

is the probability measure for the diffusively rescaled random walk starting at $z$ to hit the lower curve at $(\mathbf{s}, \mathbf{b})$ and the upper (cutoff) curve at $(\overline{\mathbf{s}}, \overline{\mathbf{b}})$. Note again that the centered random walk only takes jumps upwards of at most one step and therefore $\mathbf{B}_{\varepsilon}(\mathbf{x}) \leqslant z+\varepsilon^{-1 / 2} \mathbf{x}$. Since $\left(\mathfrak{g}_{\ell}^{\varepsilon}\right)^{\ell,-}(\mathbf{x}) \geqslant-\overline{\boldsymbol{\alpha}}-\bar{\gamma}|\ell-\mathbf{x}|$, we simply cannot have $\boldsymbol{\tau}_{\varepsilon}<1$ if $z<-N:=-\varepsilon^{-1 / 2}-\overline{\boldsymbol{\alpha}}-\bar{\gamma}|\ell-1|$, i.e. the integrations are restricted to $z \geqslant-N$.

The operators inside the norms above are rank-1, so the trace norms are the product of $L^{2}$ norms, and we get for (B.14) a bound of a constant multiple of

$$
\int p_{z}^{\varepsilon}(d \mathbf{s}, d \overline{\mathbf{s}}, d \mathbf{b}, d \overline{\mathbf{b}}) d z \mathbf{1}_{\mathbf{s}<1, \overline{\mathbf{s}}<\varepsilon n+\ell} \exp \left(F_{\varepsilon}\left(\mathbf{x}_{1}-\ell, z\right)+\bar{F}_{\varepsilon}\left(-\mathbf{x}_{2}+\ell-\overline{\mathbf{s}}, \overline{\mathbf{b}}\right)\right) .
$$

We will proceed to estimate this one; it will be clear from the proof that the same argument works for (B.13) with a few simplifications, since $\boldsymbol{\tau}_{\varepsilon}$ and $\mathbf{b}$ are under better control than $\overline{\boldsymbol{\tau}}_{\varepsilon}$ amd $\overline{\mathbf{b}}$. 
Note first of all that, in the same way as in (B.8), for $z \leqslant-\overline{\boldsymbol{\alpha}}-\bar{\gamma} \ell$, we have

$$
p_{z}^{\varepsilon}(\mathbf{s}<1) \leqslant \exp \left(-\kappa(z+\overline{\boldsymbol{\alpha}}+\bar{\gamma} \ell)^{2}\right)
$$

(and furthermore it simply vanishes if $z<-N$. as we just argued). We have the lower bound $\overline{\mathbf{b}} \geqslant-\overline{\boldsymbol{\alpha}}-\bar{\gamma}|\ell-\overline{\mathbf{s}}|$, and we claim that this is enough to get a bound

$$
\bar{F}_{\varepsilon}\left(-\mathbf{x}_{2}+\ell-\overline{\mathbf{s}}, \overline{\mathbf{b}}\right) \leqslant C(1+\log \ell)
$$

with $C<\infty$ independent of $\varepsilon$ or $\ell$. Call

$$
\overline{\mathbf{x}}_{\varepsilon}=-\mathbf{x}_{2}+\ell-\overline{\mathbf{s}}+\frac{1}{2} \varepsilon^{1 / 2} \overline{\mathbf{b}}+\frac{3}{2} \varepsilon \quad \text { and } \quad \bar{u}_{\varepsilon}=\overline{\mathbf{b}}+\varepsilon^{1 / 2}
$$

Usually, we have been breaking into cases $\overline{\mathbf{x}}_{\varepsilon}^{2}+\bar{u}_{\varepsilon} \geqslant 0$ or not, but now let us suppose that we have the stronger condition $\frac{1}{2} \overline{\mathbf{x}}_{\varepsilon}^{2}+\bar{u}_{\varepsilon} \geqslant 0$. Of course, this implies that $\overline{\mathbf{y}}_{\varepsilon}=\overline{\mathbf{x}}_{\varepsilon}^{2}+\bar{u}_{\varepsilon} \geqslant 0$, so $\nu_{1}, \nu_{2} \geqslant 0$. We have

$$
\overline{\mathbf{x}}_{\varepsilon} \overline{\mathbf{y}}_{\varepsilon}-\frac{1}{2} \overline{\mathbf{x}}_{\varepsilon}^{2} \overline{\mathbf{y}}_{\varepsilon}^{1 / 2}-\frac{1}{2} \overline{\mathbf{y}}_{\varepsilon}^{3 / 2} \leqslant 0
$$

by Young's inequality and the fact that $\overline{\mathbf{y}}_{\varepsilon} \geqslant 0$, but now the extra condition $\frac{1}{2} \overline{\mathbf{x}}_{\varepsilon}^{2}+\bar{u}_{\varepsilon} \geqslant 0$ allows us to check the non-obvious fact that

$$
\overline{\mathbf{x}}_{\varepsilon} \overline{\mathbf{y}}_{\varepsilon}-\frac{1}{3} \overline{\mathbf{x}}_{\varepsilon}^{3}-\frac{2}{3} \overline{\mathbf{y}}_{\varepsilon}^{3 / 2} \leqslant 0
$$

as well. Therefore, under these conditions, we have $\widehat{F}_{\varepsilon}\left(\overline{\mathbf{x}}_{\varepsilon}, \bar{u}_{\varepsilon}\right) \leqslant 0$. Now, there exists a $C$ depending on $\mathbf{x}_{2}, \overline{\boldsymbol{\alpha}}$ and $\bar{\gamma}$ such that, if $\overline{\mathbf{x}}_{\varepsilon} \geqslant C \varepsilon^{1 / 2}$ then, since $-\overline{\boldsymbol{\alpha}}-\bar{\gamma} \ell \bar{u}_{\varepsilon} \geqslant-\varepsilon^{-1 / 2} \overline{\mathbf{x}}_{\varepsilon}$, we can use Lemma B.2 (iii) to get the bound $C(1+\log \ell)$. On the other hand, if $\overline{\mathbf{x}}_{\varepsilon}<C \varepsilon^{1 / 2}$, we estimate

$$
\bar{F}_{\varepsilon}\left(-\mathbf{x}_{2}+\ell-\overline{\mathbf{s}}, \overline{\mathbf{b}}\right) \leqslant \widehat{F}\left(\overline{\mathbf{x}}_{\varepsilon}, \bar{u}_{\varepsilon}\right) \leqslant 0
$$

using the above argument for the last inequality and Lemma B.2 (ii) for the first, which we are allowed to use because of the condition $\overline{\mathbf{s}} \leqslant \varepsilon n+\ell$.

Alternatively, we have $\frac{1}{2} \overline{\mathbf{x}}_{\varepsilon}^{2}+\bar{u}_{\varepsilon}<0$. Because of the lower bound on $\overline{\mathbf{b}}$, there is a $C<\infty$, depending only on $\overline{\boldsymbol{\alpha}}$ and $\bar{\gamma}$, such that $\left|\overline{\mathbf{x}}_{\varepsilon}\right|^{3}+\left|\bar{u}_{\varepsilon}\right|^{3 / 2} \leqslant C$. Then, we can use Lemma B.2 (iii) when $\overline{\mathbf{x}}_{\varepsilon} \geqslant 0$, or, when $\overline{\mathbf{x}}_{\varepsilon}<0$, Lemma B.2(v) if $\overline{\mathbf{x}}_{\varepsilon}^{2}+\bar{u}_{\varepsilon}<0$ or Lemma B.2 (ii) if $\overline{\mathbf{x}}_{\varepsilon}^{2}+\bar{u}_{\varepsilon} \geqslant 0$, to prove (B.16).

Now, we consider $F_{\varepsilon}\left(\mathbf{x}_{1}-\ell, z\right)$. We claim that

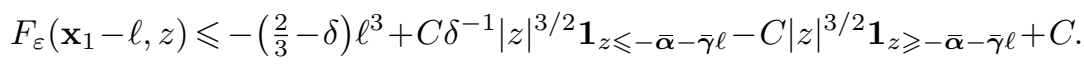

Let $\mathbf{x}_{\varepsilon}=\mathbf{x}_{1}-\ell-\frac{1}{2} \varepsilon^{1 / 2} z-\frac{1}{2} \varepsilon$ and $u_{\varepsilon}=z-\varepsilon^{1 / 2}$. Consider first the case $\frac{1}{2} \mathbf{x}_{\varepsilon}^{2}+u_{\varepsilon} \leqslant 0$. Since $z \geqslant-\overline{\boldsymbol{\alpha}}-\bar{\gamma} \ell-\varepsilon^{-1 / 2}$, this can only happen if $\ell \leqslant C \varepsilon^{-1 / 4}$ and we have $\left|\mathbf{x}_{\varepsilon}\right| \leqslant C \varepsilon^{-1 / 4}$, 
$\left|u_{\varepsilon}\right| \leqslant C \varepsilon^{-1 / 2}$ and $\left|u_{\varepsilon}\right|^{1 / 2} /\left|\mathbf{x}_{\varepsilon}\right|$ bounded so, if $\mathbf{x}_{\varepsilon}<0$, we can use Lemma B.2 (iv) to get (B.17). Suppose, on the other hand, that $\frac{1}{2} \mathbf{x}_{\varepsilon}^{2}+u_{\varepsilon} \geqslant 0$. If $-\mathbf{x}_{\varepsilon}+\sqrt{\mathbf{x}_{\varepsilon}^{2}+u_{\varepsilon}} \geqslant \varepsilon^{-1 / 2}$, by Lemma B.2(i), there is nothing to estimate. Otherwise, we have

$$
-\mathbf{x}_{\varepsilon} \leqslant-\mathbf{x}_{\varepsilon}+\sqrt{\mathbf{x}_{\varepsilon}^{2}+u_{\varepsilon}}<\varepsilon^{-1 / 2}
$$

which, together with the lower bound on $z$, gives $\varepsilon^{1 / 2} \mathbf{x}_{\varepsilon} \in(1-\sqrt{5}, 1+\sqrt{5})$ independent of $\ell$, so we can use Lemmas B.2 (ii) and (B.3), together with the facts that $\nu_{1}$ and $\nu_{2}$ are non-negative and bounded independent of $\ell$ and that $\mathbf{x}_{\varepsilon} \mathbf{y}_{\varepsilon}-\frac{1}{2} \mathbf{x}_{\varepsilon}^{2} \mathbf{y}_{\varepsilon}^{1 / 2}-\frac{1}{2} \mathbf{y}_{\varepsilon}^{3 / 2} \leqslant 0$, to get

$$
F_{\varepsilon}\left(\mathbf{x}_{1}-\ell, z\right) \leqslant \mathbf{x}_{\varepsilon} u_{\varepsilon}+\frac{2}{3} \mathbf{x}_{\varepsilon}^{3}-\frac{2}{3}\left(\mathbf{x}_{\varepsilon}^{2}+u_{\varepsilon}\right)^{3 / 2} \text {. }
$$

Using $\mathbf{x}_{\varepsilon} u_{\varepsilon} \leqslant \delta\left|\mathbf{x}_{\varepsilon}\right|^{3}+C \delta^{-1}\left|u_{\varepsilon}\right|^{3 / 2}$ and $z \geqslant-\overline{\boldsymbol{\alpha}}-\bar{\gamma} \ell-\varepsilon^{-1 / 2}$, this is bounded above by

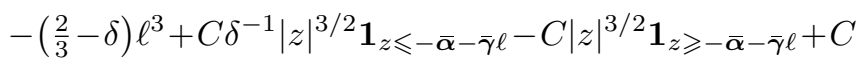

for sufficiently small $\varepsilon$. There is finally the case $\mathbf{x}_{\varepsilon} \geqslant 0$ but $\frac{1}{2} \mathbf{x}_{\varepsilon}^{2}+u_{\varepsilon}<0$. Here, $\ell$ is bounded and we can estimate by $C \log (|z|+C)$, by Lemma B.2(iii), which can be absorbed into the right-hand side of (B.17). This completes the proof of (B.17).

Now we can use (B.15)-(B.17) to see that (B.13) is bounded by

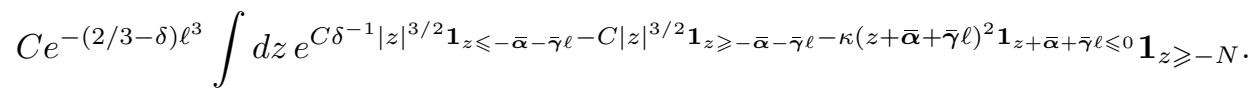

It is not hard to see that this last term is bounded by $C^{\prime} e^{-(2 / 3-\delta) \ell^{3}}$, completing the proof.

Remark B.4. Examining the argument, it is not hard to see that one should be able to get away with $\bar{\gamma}(\mathbf{x}) \geqslant-\overline{\boldsymbol{\alpha}}-\bar{\gamma}|\mathbf{x}|^{2}$, if $\bar{\gamma}$ is sufficiently small, depending on $\mathbf{t}$. From the variational formula (4.18), it is clear that $\bar{\gamma}=1 / \mathbf{t}$ is the physical barrier, but in fact the above argument breaks down at $\bar{\gamma}=c / \mathbf{t}$ with $c \approx 0.9$.In fact, for $c / \mathbf{t}<\bar{\gamma}<1 / \mathbf{t}$, one has to do a very fine estimate on an oscillatory integral in order to control things. We do not pursue it here.

Remark B.5. There is still one term not taken care of by the preceeding argument; we need to show that, for $i<j, \mathbf{x}_{i}>\mathbf{x}_{j}$ and $\mathbf{a}_{i}, \mathbf{a}_{j}$ and the scaling introduced in (3.8), as well as the scaled variables $z_{i}=2 \varepsilon^{-1} \mathbf{x}_{i}+\varepsilon^{-1 / 2}\left(u_{i}+\mathbf{a}_{i}\right)-2$ introduced in Lemma 3.5,

$$
\left\|\vartheta_{i}\left(u_{i}\right) \bar{\chi}_{\mathbf{a}_{i}}\left(u_{i}\right)\left(\varepsilon^{-1 / 2} Q^{n_{j}-n_{i}}\left(z_{i}, z_{j}\right)-e^{\left(\mathbf{x}_{i}-\mathbf{x}_{j}\right) \partial^{2}}\left(u_{i}, u_{j}\right)\right) \bar{\chi}_{\mathbf{a}_{j}}\left(u_{j}\right) \vartheta_{j}^{-1}\left(u_{j}\right)\right\|_{1} \rightarrow 0
$$

as $\varepsilon \searrow 0$. The pointwise convergence

$$
\varepsilon^{-1 / 2} Q^{n_{j}-n_{i}}\left(z_{i}, z_{j}\right) \rightarrow e^{\left(\mathbf{x}_{i}-\mathbf{x}_{j}\right) \partial^{2}}\left(u_{i}, u_{j}\right)
$$


is just a standard convergence of random walk transition probabilities to those of Brownian motion. To see that with these conjugations the convergence holds in trace norm, we write $\varepsilon^{-1 / 2} Q^{n}-e^{\mathbf{x} \partial^{2}}$ as

$$
\begin{aligned}
& \frac{1}{2}\left(\varepsilon^{-1 / 2} Q^{\lfloor n / 2\rfloor}+e^{\mathbf{x} \partial^{2} / 2}\right)\left(\varepsilon^{-1 / 2} Q^{\lceil n / 2\rceil}-e^{\mathbf{x} \partial^{2} / 2}\right) \\
& \quad+\frac{1}{2}\left(\varepsilon^{-1 / 2} Q^{\lfloor n / 2\rfloor}-e^{\mathbf{x} \partial^{2} / 2}\right)\left(\varepsilon^{-1 / 2} Q^{\lceil n / 2\rceil}+e^{\mathbf{x} \partial^{2} / 2}\right) .
\end{aligned}
$$

We can estimate the trace norm of each of the two terms as in the proof of Lemma A.2 of [9]. Introducing the factors $\vartheta_{i}$ and $\vartheta_{j}^{-1}$, and bounding the trace norm of the product by the product of the Hilbert-Schmidt norms we get, for twice the first term, a bound by the square root of

$$
\begin{array}{rl}
\int_{\mathbb{R}^{2}} & d u_{i} d z\left(\varepsilon^{-1 / 2} Q^{\left\lfloor\left(n_{j}-n_{i}\right) / 2\right\rfloor}+e^{\left(\mathbf{x}_{i}-\mathbf{x}_{j}\right) \partial^{2} / 2}\right)^{2}\left(u_{i}-z\right) \frac{\left(1+u_{i}^{2}\right)^{4 i}}{\left(1+z^{2}\right)^{2(i+j)}} \\
& \times \int_{\mathbb{R}^{2}} d u_{j} d z\left(\varepsilon^{-1 / 2} Q^{\left\lceil\left(n_{j}-n_{i}\right) / 2\right\rceil}-e^{\left(\mathbf{x}_{i}-\mathbf{x}_{j}\right) \partial^{2} / 2}\right)^{2}\left(z-u_{j}\right) \frac{\left(1+z^{2}\right)^{2(i+j)}}{\left(1+u_{j}^{2}\right)^{4 j}},
\end{array}
$$

which goes to zero as $\varepsilon \searrow 0$. The other term is essentially the same.

\section{B.2. Convergence}

We now explain how the above trace estimates prove the convergence of the TASEP approximations to their continuum versions. The same argument shows the continuity of the limiting kernels from UC to the trace class.

We want to show that

$$
\left(\mathbf{S}_{-1, \mathbf{x}}^{\varepsilon}\right)^{*} \overline{\mathbf{S}}_{-1,-\mathbf{x}}^{\varepsilon, \mathrm{epi}\left(\mathfrak{g}^{\varepsilon}\right)} \underset{\varepsilon \rightarrow 0}{\longrightarrow}\left(\mathbf{S}_{-1, \mathbf{x}}\right)^{*} \overline{\mathbf{S}}_{-1,-\mathbf{x}}^{\mathrm{epi}(\mathfrak{g})}
$$

in trace norm as $\mathfrak{g}^{\varepsilon} \rightarrow \mathfrak{g}$ in $\operatorname{LC}[0, \infty)$. More explicitly, if $\boldsymbol{\tau}^{\varepsilon}$ is the hitting time of $\mathfrak{g}^{\varepsilon}$ by $\mathbf{B}_{\varepsilon}$, we want

$$
\begin{aligned}
& \int_{\substack{\mathbf{s} \in[0, \infty) \\
\mathbf{b}, z \in \mathbb{R}}} d z \mathbb{P}_{\mathbf{B}_{\varepsilon}(0)=z}\left(\boldsymbol{\tau}_{\varepsilon} \in d \mathbf{s}, \mathbf{B}_{\varepsilon}\left(\tau_{\varepsilon}\right) \in d \mathbf{b}\right) \mathbf{S}_{-1, \mathbf{x}}^{\varepsilon}\left(z, z_{1}\right) \overline{\mathbf{S}}_{-1,-\mathbf{x}-\mathbf{s}}^{\varepsilon}\left(\mathbf{b}, z_{2}\right) \\
& \quad \rightarrow \int_{\substack{\mathbf{s} \in[0, \infty) \\
\mathbf{b}, z \in \mathbb{R}}} d z \mathbb{P}_{\mathbf{B}(0)=z}(\boldsymbol{\tau} \in d \mathbf{s}, \mathbf{B}(\tau) \in d \mathbf{b}) \mathbf{S}_{-1, \mathbf{x}}\left(z, z_{1}\right) \overline{\mathbf{S}}_{-1,-\mathbf{x}-\mathbf{s}}\left(\mathbf{b}, z_{2}\right),
\end{aligned}
$$

as integral operators in the trace class. First, we claim that

$$
\mathbb{P}_{\mathbf{B}_{\varepsilon}(0)=z}\left(\boldsymbol{\tau}_{\varepsilon} \in d \mathbf{s}, \mathbf{B}_{\varepsilon}\left(\boldsymbol{\tau}_{\varepsilon}\right) \in d \mathbf{b}\right) \rightarrow \mathbb{P}_{\mathbf{B}(0)=z}(\boldsymbol{\tau} \in d \mathbf{s}, \mathbf{B}(\boldsymbol{\tau}) \in d \mathbf{b})
$$


as measures. By Donsker's invariance principle $\mathbf{B}_{\varepsilon} \rightarrow \mathbf{B}$ uniformly on compact sets. By Proposition 3.2, if $z<\mathfrak{g}(0)$ we have (B.20), and furthermore the convergence is uniform over sets of locally bounded Hölder $\beta$-norm. On the other hand, if $z>\mathfrak{g}(0)$ then, from our assumption, $z>\mathfrak{g}^{\varepsilon}(0)$ for sufficiently small $\varepsilon$, and then $\boldsymbol{\tau}_{\varepsilon}=\boldsymbol{\tau}=0$, so the convergence as measures also happens for $z>\mathfrak{g}(0)$.

By the estimates proved in the previous section, one can restrict the integration in (B.19) to $\mathbf{s}, \mathbf{b}$ and $z$ in compact intervals, the complementary integration being uniformly small in $\varepsilon$ in trace norm. Since we are now on a compact interval of $z$, we can normalize to make

$$
\mu_{\varepsilon}=c_{\varepsilon} \mathbb{P}_{\mathbf{B}_{\varepsilon}(0)=z}\left(\boldsymbol{\tau}_{\varepsilon} \in d \mathbf{s}, \mathbf{B}_{\varepsilon}\left(\tau_{\varepsilon}\right) \in d \mathbf{b}\right) d z \quad \text { and } \quad \mu=c \mathbb{P}_{\mathbf{B}(0)=z}(\boldsymbol{\tau} \in d \mathbf{s}, \mathbf{B}(\tau) \in d \mathbf{b}) d z
$$

probability measures. Taking $\mathscr{B}$ as the trace class, the convergence then follows by

$$
\left\|\int\left(f_{\varepsilon}-f\right) d \mu_{\varepsilon}\right\| \leqslant \int\left\|f_{\varepsilon}-f\right\| d \mu_{\varepsilon},
$$

and the following fact, which is presumably well known, but whose proof is easier than searching for a reference.

Proposition B.6. Let $\mu_{n}, n=1,2, \ldots$, and $\mu$ be probability measures on a Polish space $\Omega$, with $\mu_{n}$ converging weakly to $\mu$ as $n \rightarrow \infty$. Let $f$ be a bounded continuous function from $\Omega$ into a Banach space $\mathscr{B}$. Then,

$$
\int f d \mu_{n} \rightarrow \int f d \mu .
$$

Proof. The $\mu_{n}$ are tight, so, by throwing away a set of uniformly small measure, we may assume that $\Omega$ is compact. By the Skorokhod representation theorem, there exist random variables $X_{n}, n=1,2, \ldots$, and $X$, distributed according to $\mu_{n}$ and $\mu$ with $X_{n} \rightarrow X$ almost surely. Let $\varepsilon>0$. Since $\Omega$ is compact, there is $\delta>0$ such that $\left\|f(\omega)-f\left(\omega^{\prime}\right)\right\|<\frac{1}{2} \varepsilon$ whenever $d\left(\omega, \omega^{\prime}\right)<\delta$. So,

$$
\left\|\mathbb{E}\left[f\left(X_{n}\right)-f(X)\right]\right\| \leqslant\|f\|_{\infty} \mathbb{P}\left(d\left(X_{n}, X\right) \geqslant \delta\right)+\frac{1}{2} \varepsilon<\varepsilon
$$

for $n$ sufficiently large.

This proves (B.18) which, in view of the discussion around (3.21), yields Proposition 3.6, i.e. the convergence of the TASEP finite-dimensional distributions to those of the fixed point in the case of 1-sided initial data. The extension 2-sided initial data (i.e. Theorem 3.13) is done as explained in $\S 3.4$, the main tool being the finite propagation speed result, Lemma 3.4, proved in the next section. 


\section{Appendix C. Finite propagation speed and regularity}

\section{C.1. Finite propagation speed}

We will now prove Lemma 3.4. First of all, note that by a union bound it suffices to prove the result for $m=1$. The resulting constants will then depend on $m$. In reality, the constants are independent of $m$ if all the points $\mathbf{x}_{i}$ are bounded above. But we do not use it anywhere, so we just give the simplest proof which suffices for the results of this article. For simplicity, we will also set $\mathbf{t}=1$ and, by shifting the initial data, we can set $\mathbf{a}=0$.

Recall that we are given initial data $X_{0}^{\varepsilon}$ satisfying (3.4) in the UC topology, which in particular means that we have a fixed $\overline{\boldsymbol{\alpha}}, \overline{\boldsymbol{\gamma}}<\infty$ such that $\mathfrak{h}_{0}^{\varepsilon}(\mathbf{y}) \leqslant \overline{\boldsymbol{\alpha}}+\overline{\boldsymbol{\gamma}}|\mathbf{y}|$ for all $\varepsilon>0$. Then, we make the UC cutoff, replacing it by $X_{0}^{\varepsilon, L}$ in which all the particles with label less than or equal to $-\varepsilon^{-1} L^{\prime}$ are moved to $\infty$. The corresponding rescaled height function is denoted by $\mathfrak{h}_{0}^{\varepsilon, L}$ and we choose $L^{\prime} \sim \frac{1}{2} L$ in $\varepsilon \mathbb{Z}$ so that this function has been replaced by a straight line with slope $-2 \varepsilon^{-1 / 2}$ to the right of $L$ (see Definition 3.3). We will prove that the difference of (3.7) with $m=1$, that is

$$
\mathbb{P}\left(X_{2 \varepsilon^{-3 / 2} \mathbf{t}}\left(\frac{1}{2} \varepsilon^{-3 / 2} \mathbf{t}-\varepsilon^{-1} \mathbf{x}-\frac{1}{2} \varepsilon^{-1 / 2} \mathbf{a}+1\right)>2 \varepsilon^{-1} \mathbf{x}-2\right)
$$

for some fixed $\mathbf{a}$ and $\mathbf{x}$, computed with initial data $X_{0}^{\varepsilon, L-1}$ and initial data $X_{0}^{\varepsilon, L}$, is less than $C e^{-c L^{3}}$. Then, one just sums over integers $L \geqslant L_{0}$ to get Lemma 3.4.

Note that, to keep the notation as simple as possible, we may assume in this subsection that $\mathbf{x}=0$, or, to make the notation even simpler, $\mathbf{x}=\varepsilon$ because this makes $a=0$ in (3.8). From translation invariance, we do not actually lose generality by assuming this.

To bound the difference of probabilities we use the Fredholm determinant formula and (A.2), which reduces the problem to estimating the trace norm of

$$
\bar{\chi}_{0}\left(\mathcal{S}_{-t,-n}\right)^{*}\left(\overline{\mathcal{S}}_{-t, n}^{\mathrm{epi}\left(X_{0}^{\varepsilon, L-1}\right)}-\overline{\mathcal{S}}_{-t, n}^{\mathrm{epi}\left(X_{0}^{\varepsilon, L}\right)}\right) \bar{\chi}_{0},
$$

with $t=2 \varepsilon^{-3 / 2}$ and $n=\frac{1}{2} \varepsilon^{-3 / 2}$. It is more convenient $\mathrm{t} \mathrm{o}$ use the cutoff position as a frame of reference so we translate the cutoff rescaled height configurations to the left by a macroscopic distance $L$ so that the rightmost (non-infinite) particle of $\theta_{-\varepsilon^{-1} L^{\prime}} X_{0}^{\varepsilon, L}$ has label 1 and $\theta_{-\varepsilon^{-1} L^{\prime}} X_{0}^{\varepsilon, L-1}$ is the same configuration, except that about $\frac{1}{2} \varepsilon^{-1}$ of the rightmost particles have been moved to $+\infty$. The shifted macroscopic height functions have been replaced by a line of slope $-2 \varepsilon^{-1 / 2}$ to the right of zero in the first case, and to the right of -1 in the second case. To view the system from the same position as before, we have to replace $n$ by $n+\varepsilon^{-1} L^{\prime}$, and so the problem comes down to bounding the trace norm of

$$
\bar{\chi}_{0}\left(\mathcal{S}_{-t,-n-\varepsilon^{-1} L^{\prime}}\right)^{*}\left(\overline{\mathcal{S}}_{-t, n+\varepsilon^{-1} L^{\prime}}^{\mathrm{epi}\left(\theta_{-\varepsilon^{-1}} X_{0}^{\varepsilon, L-1}\right)}-\overline{\mathcal{S}}_{-t, n+\varepsilon^{-1} L^{\prime}}^{\mathrm{epi}\left(\theta_{-\varepsilon^{-1}} X_{0}^{\varepsilon, L}\right)}\right) \bar{\chi}_{0} .
$$


In the language of the rescaled kernels from Lemma 3.5, we need to bound the trace norm of

$$
\bar{\chi}_{0}\left(\mathbf{S}_{-1,-L}^{\varepsilon}\right)^{*}\left(\overline{\mathbf{S}}_{-1, L}^{\varepsilon, \operatorname{epi}\left(-\left(\theta_{L} \mathfrak{h}_{0}^{\varepsilon, L-1}\right)^{-}\right)}-\overline{\mathbf{S}}_{-1, L}^{\varepsilon, \operatorname{epi}\left(-\left(\theta_{L} \mathfrak{h}_{0}^{\varepsilon, L}\right)^{-}\right)}\right) \bar{\chi}_{0}
$$

but this is exactly Lemma B.3.

Remark C.1. At the level of the fixed point, one has immediately from the 1-point version of the variational formula,

$$
\mathfrak{h}(1,0) \stackrel{\text { dist }}{=} \sup _{\mathbf{y} \in \mathbb{R}}\left\{\mathcal{A}_{2}(\mathbf{y})-\mathbf{y}^{2}+\mathfrak{h}_{0}(\mathbf{y})\right\}
$$

with $\mathcal{A}_{2}(\mathbf{y})$ the Airy process (see Remark 4.19), that replacing $\mathfrak{h}_{0}$ by the cutoff

$$
\mathfrak{h}_{0}^{L}(\mathbf{y})=\mathfrak{h}_{0}(\mathbf{y})-\infty \mathbf{1}_{\mathbf{y}>L},
$$

affects the value of $\mathfrak{h}(1,0)$ only if the supremum is achieved at $\mathbf{y}>L$. As $\mathfrak{h}_{0}(\mathbf{y}) \leqslant \overline{\boldsymbol{\alpha}}+\bar{\gamma}|\mathbf{y}|$, this is essentially controlled by the probability that $\mathcal{A}_{2}(L) \geqslant L^{2}-\bar{\gamma} L-\overline{\boldsymbol{\alpha}}$. Since $\mathcal{A}_{2}(L)$ has the GUE Tracy-Widom distribution, this is roughly $\exp \left\{-\frac{2}{3} L^{3}\right\}$. It is not hard to make this argument rigorous. Since TASEP satisfies a microscopic version of the variational formula, one could provide an alternate proof of the finite propagation speed following the same argument. It reduces to a large deviation bound for the tail probability in the microscopic analogue of the Airy process. This can be proved with our formulas, and leads to similar computations as our proof of the cutoff. We did it via Lemma B.3, because we need it later as well in the proof of regularity.

\section{C.2. Regularity}

Next, we obtain the necessary tightness on $\mathfrak{h}^{\varepsilon}(\mathbf{t}, \mathbf{x})$ by obtaining uniform bounds on the local Hölder norm $\beta<\frac{1}{2}$. Note that we are working at $\mathbf{t}$ fixed, and the bounds are as functions of $\mathbf{x}$, so we will assume in the rest of the section that $\mathbf{t}=1$; other times can be obtained analogously, or alternatively by scaling. We start with a well-known version of the Kolmogorov continuity theorem.

Lemma C.2. Let $\mathfrak{h}(\mathbf{x})$ be a stochastic process defined for $\mathbf{x}$ in an interval $[-M, M] \subseteq$ $\mathbb{R}$ such that, for some $p>1$ and $\alpha>0$,

$$
\mathbb{E}\left[|\mathfrak{h}(\mathbf{x})-\mathfrak{h}(\mathbf{y})|^{p}\right] \leqslant C|\mathbf{x}-\mathbf{y}|^{1+\alpha}
$$

Then, for every $\beta<\alpha / p$, there is a $y \bar{C}=\bar{C}(p, \alpha, \beta, C)$ such that, for the local Hölder norm defined in (3.6),

$$
\mathbb{P}\left(\|\mathfrak{h}\|_{\beta,[-M, M]} \geqslant R\right) \leqslant \bar{C} R^{-p} .
$$


We want to obtain an estimate like (C.1) for our process, but we have only access to cumulative distribution functions. We can use the following lemma.

Lemma C.3. Let $H_{1}$ and $H_{2}$ be random variables. Then, for $p \geqslant 2$,

$$
\mathbb{E}\left[\left|H_{1}-H_{2}\right|^{p} \mathbf{1}_{H_{1}>H_{2}}\right]=p(p-1) \int_{-\infty}^{\infty} \int_{-\infty}^{\infty} d \mathbf{a} d \mathbf{b}|\mathbf{a}-\mathbf{b}|^{p-2} \mathbf{1}_{\mathbf{a}>\mathbf{b}} \mathbb{P}\left(H_{1}>\mathbf{a}, H_{2} \leqslant \mathbf{b}\right) .
$$

Proof. Since the integrand is positive, the right-hand side of (C.3) can be rewritten using Fubini's theorem as

$$
\mathbb{E}\left[\int_{H_{2}}^{H_{1}} d h_{2} \int_{h_{2}}^{H_{1}} d h_{1} p(p-1)\left(h_{1}-h_{2}\right)^{p-2} \mathbf{1}_{H_{1}>H_{2}}\right]
$$

Performing the integrations inside the expectation gives the left-hand side.

Proof of Theorem 4.13. We want to use the previous lemma, but there is a little problem. We have formulas for

$$
\mathbb{P}(\mathfrak{h}(\mathbf{x})>\mathbf{a}, \mathfrak{h}(\mathbf{y}) \leqslant \mathbf{b})=\mathbb{P}(\mathfrak{h}(\mathbf{y}) \leqslant \mathbf{b})-\mathbb{P}(\mathfrak{h}(\mathbf{x}) \leqslant \mathbf{a}, \mathfrak{h}(\mathbf{y}) \leqslant \mathbf{b})
$$

in terms of differences of Fredholm determinants, which can be estimated by the trace norm of the difference of kernels. If $\mathbf{x}$ is close to $\mathbf{y}$, the difference is small, as desired. However, it is not straightforward to get the needed decay as $\mathbf{a}, \mathbf{b} \rightarrow \pm \infty$ (even if one knows the tails $\mathbb{P}(\mathfrak{h}(\mathbf{x})>\mathbf{a})$ and $\mathbb{P}(\mathfrak{h}(\mathbf{y}) \leqslant \mathbf{b})$ decay exponentially, it would still mean one had to control the difference of determinants on a range $c \log |\mathbf{a}-\mathbf{b}|^{-1}$ for $|\mathbf{a}-\mathbf{b}|$ small, which is non-trivial.) We get around this with a simple trick. Let $\mathfrak{h}_{N}(\mathbf{x})=(\mathfrak{h}(\mathbf{x}) \wedge N) \vee(-N)$ be the cutoff of $\mathfrak{h}$ at $\pm N$. Applying Lemma C.3 to $H_{1}=\mathfrak{h}_{N}^{\varepsilon}(\mathbf{x})$ and $H_{2}=\mathfrak{h}_{N}^{\varepsilon}(\mathbf{y})$, we have, for fixed $\mathbf{t}$ and $p \geqslant 2$,

$$
\begin{aligned}
& \mathbb{E}\left[\left|\mathfrak{h}_{N}^{\varepsilon}(\mathbf{x})-\mathfrak{h}_{N}^{\varepsilon}(\mathbf{y})\right|^{p}\right] \\
& \quad=p(p-1) \int_{-N}^{N} \int_{-N}^{N} d \mathbf{a} d \mathbf{b}|\mathbf{a}-\mathbf{b}|^{p-2}\left[F_{\mathbf{x}}^{\varepsilon}(\mathbf{a}) \mathbf{1}_{\mathbf{a}<\mathbf{b}}+F_{\mathbf{x}}^{\varepsilon}(\mathbf{b}) \mathbf{1}_{\mathbf{a} \geqslant \mathbf{b}}-F_{\mathbf{x}, \mathbf{y}}^{\varepsilon}(\mathbf{a}, \mathbf{b})\right],
\end{aligned}
$$

where $F^{\varepsilon}$ are the 1- and 2-point cumulative distribution functions of the 1:2:3 rescaled TASEP height functions. Now, suppose we find some $p>1, \alpha>0$ and $C=C(N)$ such that the right-hand side is bounded by $C|\mathbf{x}-\mathbf{y}|^{1+\alpha}$ independent of $\varepsilon>0$. Suppose also that

$$
\limsup _{N \rightarrow \infty} \limsup _{\varepsilon \rightarrow 0} \mathbb{P}\left(\sup _{\mathbf{x} \in[-M, M]}\left|\mathfrak{h}^{\varepsilon}(\mathbf{x})\right| \geqslant N\right)=0
$$

Then, we have (4.12) because, if $\sup _{\mathbf{x} \in[-M, M]}\left|\mathfrak{h}^{\varepsilon}(\mathbf{x})\right| \leqslant N$, then $\mathfrak{h}_{N}^{\varepsilon}=\mathfrak{h}^{\varepsilon}$ on $[-M, M]$. 
To see that (C.4) holds, we note that we have assumed that $\mathfrak{h}^{\varepsilon}(0, \mathbf{x}) \leqslant C(1+|\mathbf{x}|)$. We may also assume, without loss of generality, that $\mathfrak{h}^{\varepsilon}\left(0, \mathbf{x}_{\varepsilon}\right) \geqslant \ell>-\infty$ independent of $\varepsilon$ for some $\mathbf{x}_{\varepsilon} \rightarrow \mathbf{x}_{0}$, since the $\mathfrak{h}^{\varepsilon}$ have been assumed to converge to some $\mathfrak{h}$ in UC, and we may assume that $\mathfrak{h}\left(\mathbf{x}_{0}\right)>-\infty$ for some $\mathbf{x}_{0}$. Therefore, we can bound $\mathfrak{h}^{\varepsilon}(1, \mathbf{x})$ above by the maximum of two rescaled TASEP height functions, one starting with $C(1+x)$ and one starting with $C(1-x)$. For each, we can estimate the probability that the height profile is greater than $N$ anywhere on $[-M, M]$ by cutting the initial data at $L$, using the exact formulas (e.g. from Example 2.10), and showing that the bound does not depend on $L$. This is fairly standard, so we omit the details. This shows that

$$
\underset{N \rightarrow \infty}{\limsup } \limsup _{\varepsilon \rightarrow 0} \mathbb{P}\left(\sup _{\mathbf{x} \in[-M, M]} \mathfrak{h}^{\varepsilon}(\mathbf{x}) \geqslant N\right)=0 .
$$

For the other direction, we bound $\mathfrak{h}^{\varepsilon}(\mathbf{t}, \mathbf{x})$ below by the narrow wedge solution $\underline{h}^{\varepsilon}(\mathbf{t}, \mathbf{x})$ centered at $\mathbf{x}_{\varepsilon}$. By results of Johansson [34], $\underline{\mathfrak{h}}^{\varepsilon}(1, \mathbf{x})+\left(\mathbf{x}-\mathbf{x}_{\varepsilon}\right)^{2}$ converges uniformly on compact sets to the (rescaled) Airy 2 process. In particular,

$$
\limsup _{N \rightarrow \infty} \limsup _{\varepsilon \rightarrow 0} \mathbb{P}\left(\sup _{\mathbf{x} \in[-M, M]} \mathfrak{h}^{\varepsilon}(\mathbf{x}) \leqslant-N\right)=0 .
$$

This reduces the problem to showing that, for $\mathbf{x}_{1}<\mathbf{x}_{2}$,

$$
\int_{-N \leqslant \mathbf{a}_{1}<\mathbf{a}_{2} \leqslant N} d \mathbf{a}_{1} d \mathbf{a}_{2}\left|\mathbf{a}_{1}-\mathbf{a}_{2}\right|^{p-2}\left[F_{\mathbf{x}_{1}}^{\varepsilon}\left(\mathbf{a}_{1}\right)-F_{\mathbf{x}_{1}, \mathbf{x}_{2}}^{\varepsilon}\left(\mathbf{a}_{1}, \mathbf{a}_{2}\right)\right] \leqslant C(N)\left|\mathbf{x}_{1}-\mathbf{x}_{2}\right|^{1+\alpha} .
$$

It may appear this is not general enough because $\mathbf{x}_{1}<\mathbf{x}_{2}$ and $\mathbf{a}_{1}<\mathbf{a}_{2}$, but our proof will apply equally well to the process with spatially flipped initial data $\mathfrak{h}_{0}(-\mathbf{x})$, which restores the symmetry. There is one last issue which is that we only have nice formulas for these cumulative distributions, when there is a rightmost particle. So we move all the particles to the right of $\varepsilon^{-1} L$ to $\infty$, giving a cutoff height function $\mathfrak{h}_{0}^{\varepsilon, L}$ and corresponding 1 - and 2-point distribution functions $F_{\mathbf{x}}^{\varepsilon, L}$ and $F_{\mathbf{x}, \mathbf{y}}^{\varepsilon, L}$, and we need the constant $C(N)$ in (C.5) to be independent of $L$.

From (3.7), we have

$$
F_{\mathbf{x}_{1}, \mathbf{x}_{2}}^{\varepsilon, L}\left(\mathbf{a}_{1}, \mathbf{a}_{2}\right)=\mathbb{P}_{X_{0}^{\varepsilon, L}}\left(X_{2 \varepsilon^{-3 / 2} \mathbf{t}}\left(n_{i}\right)>2 \varepsilon^{-1} \mathbf{x}_{i}-2, i=1,2\right),
$$

with $n_{i}=\frac{1}{2} \varepsilon^{-3 / 2} \mathbf{t}-\varepsilon^{-1} \mathbf{x}_{i}-\frac{1}{2} \varepsilon^{-1 / 2} \mathbf{a}_{i}+1$, and where $X_{0}^{\varepsilon, L}$ is the cutoff TASEP initial data as in Definition 3.3. By translation invariance (2.33), this is the same as

$$
\begin{aligned}
& \mathbb{P}_{\widetilde{X}_{0}^{\varepsilon, L}}\left(X_{2 \varepsilon^{-3 / G 2} \mathbf{t}}\left(\tilde{n}_{i}\right)>2 \varepsilon^{-1} \mathbf{x}_{i}-2 \text { for } i=1,2\right) \\
& \quad=\operatorname{det}\left(I-\left(\mathcal{S}_{-t,-\tilde{n}_{1}}\right)^{*} \overline{\mathcal{S}}_{-t, \tilde{n}_{1}}^{\operatorname{epi}\left(\tilde{X}_{0}^{\varepsilon, L}\right)}\left(I-Q^{n_{2}-n_{1}} \chi_{2 \varepsilon^{-1} \mathbf{x}_{2}-2} Q^{n_{1}-n_{2}} \chi_{2 \varepsilon^{-1} \mathbf{x}_{1}-2}\right)\right) \\
& \quad=\operatorname{det}\left(I-\left(\mathcal{S}_{-t,-\tilde{n}_{1}}\right)^{*} \overline{\mathcal{S}}_{-t, \tilde{n}_{1}}^{\operatorname{epi}\left(\widetilde{X}_{0}^{\varepsilon, L}\right)}\left(Q^{n_{2}-n_{1}} \bar{\chi}_{2 \varepsilon^{-1} \mathbf{x}_{2}-2} Q^{n_{1}-n_{2}} \chi_{2 \varepsilon^{-1} \mathbf{x}_{1}-2}+\bar{\chi}_{2 \varepsilon^{-1} \mathbf{x}_{1}-2}\right)\right),
\end{aligned}
$$


where $\widetilde{X}_{0}^{\varepsilon, L}=\theta_{-\varepsilon^{-1} L} X_{0}^{\varepsilon, L}, \tilde{n}_{i}=n_{i}+\varepsilon^{-1} L$, and the first equality is from Theorem 2.6 and (2.12) (note that $n_{1}>n_{2}$ for small $\varepsilon$ ). Since $\overline{\mathcal{S}}_{-t, \tilde{n}_{1}}^{\operatorname{epi}\left(\tilde{X}^{\varepsilon, L}\right)} Q^{n_{2}-n_{1}}=\overline{\mathcal{S}}_{-t, \tilde{n}_{2}}^{\operatorname{epi}\left(\tilde{X}_{0}^{\varepsilon, L}\right)}$, scaling the variables in the determinant as in $\S 3.3$, and noting that the scaled version of $\tilde{X}_{0}^{\varepsilon, L}$ converges to $\theta_{L} \mathfrak{h}_{0}^{L}(\mathbf{x})$ yields

$$
F_{\mathbf{x}_{1}, \mathbf{x}_{2}}^{\varepsilon, L}\left(\mathbf{a}_{1}, \mathbf{a}_{2}\right)=\operatorname{det}\left(\mathbf{I}-\mathbf{A}_{\mathbf{x}_{1}, \mathbf{a}_{1} ; \mathbf{x}_{2}, \mathbf{a}_{2}}\right),
$$

where

$$
\begin{aligned}
& \mathbf{A}_{\mathbf{x}_{1}, \mathbf{a}_{1} ; \mathbf{x}_{2}, \mathbf{a}_{2}} \\
& \quad=\bar{\chi}_{-\mathbf{a}_{1}}\left[\left(\mathbf{S}_{-\mathbf{t}, \mathbf{x}_{1}-L}^{\varepsilon}\right)^{*} \overline{\mathbf{S}}_{-\mathbf{t},-\mathbf{x}_{2}+L}^{\varepsilon, \operatorname{epi}\left(-\left(\theta_{L} \mathfrak{h}_{0}\right)^{-}\right)} \bar{\chi}_{-\mathbf{a}_{2}} \widetilde{Q}_{\mathbf{x}_{2}-\mathbf{x}_{1}}^{\varepsilon} \chi_{-\mathbf{a}_{1}}+\left(\mathbf{S}_{-\mathbf{t}, \mathbf{x}_{1}-L}^{\varepsilon}\right)^{*} \overline{\mathbf{S}}_{-\mathbf{t},-\mathbf{x}_{1}+L}^{\varepsilon, \operatorname{epi}\left(-\left(\theta_{L} \mathfrak{h}_{0}\right)^{-}\right)} \bar{\chi}_{-\mathbf{a}_{1}}\right],
\end{aligned}
$$

with

$$
\widetilde{Q}_{\mathbf{x}_{2}-\mathbf{x}_{1}}^{\varepsilon}\left(u_{2}, u_{1}\right)=\varepsilon^{-1 / 2} Q^{n_{1}-n_{2}}\left(z_{2}, z_{1}\right),
$$

and

$$
z_{i}=2 \varepsilon^{-1} \mathbf{x}_{i}+\varepsilon^{-1 / 2}\left(u_{i}+\mathbf{a}_{i}\right)-2 .
$$

Note that $\widetilde{Q}_{\mathbf{x}_{2}-\mathbf{x}_{1}}^{\varepsilon}\left(u_{2}, u_{1}\right)=\widetilde{Q}_{\mathbf{x}_{2}-\mathbf{x}_{1}}^{\varepsilon}\left(u_{2}-u_{1}\right)$ are transition densities of a centered, diffusively rescaled geometric random walk $\mathbf{B}_{\varepsilon}$.

We need to estimate $F_{\mathbf{x}_{1}}^{\varepsilon, L}\left(\mathbf{a}_{1}\right)-F_{\mathbf{x}_{1}, \mathbf{x}_{2}}^{\varepsilon, L}\left(\mathbf{a}_{1}, \mathbf{a}_{2}\right)$, which by (A.2) is controlled by the trace norm

$$
\left\|\mathbf{A}_{\mathbf{x}_{1}, \mathbf{a}_{1} ; \mathbf{x}_{1}, \mathbf{a}_{1}}-\mathbf{A}_{\mathbf{x}_{1}, \mathbf{a}_{1} ; \mathbf{x}_{2}, \mathbf{a}_{2}}\right\|_{1}=\left\|\bar{\chi}_{-\mathbf{a}_{1}}\left(\mathbf{S}_{-\mathbf{t}, \mathbf{x}_{1}-L}^{\varepsilon}\right)^{*} \overline{\mathbf{S}}_{-\mathbf{t},-\mathbf{x}_{2}+L}^{\varepsilon, e p i\left(-\left(\theta_{L} \mathfrak{h}_{0}\right)^{-}\right)} \bar{\chi}_{-\mathbf{a}_{2}} \widetilde{Q}_{\mathbf{x}_{2}-\mathbf{x}_{1}}^{\varepsilon} \chi_{-\mathbf{a}_{1}}\right\|_{1} .
$$

From Proposition B.1 we have

$$
\left\|\bar{\chi}_{-\mathbf{a}_{1}}\left(\mathbf{S}_{-\mathbf{t}, \mathbf{x}_{1}-L}^{\varepsilon}\right)^{*} \overline{\mathbf{S}}_{-\mathbf{t},-\mathbf{x}_{2}+L}^{\varepsilon, \operatorname{epi}\left(-\left(\theta_{L} \mathfrak{h}_{0}\right)^{-}\right)} \bar{\chi}_{-\mathbf{a}_{2}}\right\|_{1} \leqslant C .
$$

Since $-\mathbf{a}_{2}<-\mathbf{a}_{1}$, we also have by Schwartz's inequality,

$$
\left\|\bar{\chi}_{-\mathbf{a}_{2}} \widetilde{Q}_{\mathbf{x}_{2}-\mathbf{x}_{1}}^{\varepsilon} \chi_{-\mathbf{a}_{1}}\right\|_{\text {op }} \leqslant \int_{|y| \geqslant\left|\mathbf{a}_{2}-\mathbf{a}_{1}\right|} \widetilde{Q}_{\mathbf{x}_{2}-\mathbf{x}_{1}}^{\varepsilon}(y) d y
$$

where $\|\cdot\|_{\mathrm{op}}$ is the operator norm and we are using $\|A B\|_{1} \leqslant\|A\|_{\text {op }}\|B\|_{1}$. When we plug this estimate into the left-hand side of (C.5), we get $2 N \mathbb{E}\left[\left|\mathbf{B}_{\varepsilon}\left(\mathbf{x}_{2}-\mathbf{x}_{1}\right)\right|^{p-1}\right]$. The $2 N$ comes from the integral over $\mathbf{a}_{1}$, and we lose a power in the exponent because of the estimate in (C.6) which is by a transition probability instead of the cumulative functions. At any rate, we have $2 N \mathbb{E}\left[\left|\mathbf{B}_{\varepsilon}\left(\mathbf{x}_{2}-\mathbf{x}_{1}\right)\right|^{p-1}\right] \leqslant C(N, p)\left|\mathbf{x}_{2}-\mathbf{x}_{1}\right|^{(p-1) / 2}$. So, we have proved (C.5) with $\alpha=\frac{1}{2}(p-3)$, which means that we have (C.2) with $\beta=\frac{1}{2}-\frac{3}{2 p}$, for any $p \geqslant 2$ (though with a constant $\bar{C}$ getting worse as $p \rightarrow \infty$ ). 


\section{Appendix D. Path integral formulas}

\section{D.1. An alternative version of [8, Theorem 3.3]}

Consider a measure space $(X, \mu)$ and fix $t_{1}<\ldots<t_{n}$. In $[8, \S 3]$ a very general setting is described on which the Fredholm determinant of certain extended kernels, acting on $L^{2}\left(\left\{t_{1}, \ldots, t_{n}\right\} \times X\right)$, can be turned into the Fredholm determinant of what they call a path integral kernel, which acts on $L^{2}(X)$. The result proved in that paper can be applied to a variety of processes, and in particular yields the path integral formula for the KPZ fixed point given in Proposition 4.3. However, the TASEP extended kernel does not fit into the setting of that paper. The purpose of this subsection is thus to provide a suitable version of [8, Theorem 3.3]. We do not strive for the greatest possible generality, but instead state a relatively simple variation of [8] which is enough to obtain the TASEP path integral formula, which in turn is proved in the next subsection.

Let $\mathcal{M}(X)$ be the space of real-valued measurable functions on $X$. We are given a collection of integral operators on subspaces $\mathcal{D}_{\mathcal{W}}^{+}, \mathcal{R}_{\mathcal{W}}^{+}, \mathcal{D}_{\mathcal{W}}^{-}, \mathcal{R}_{\mathcal{W}}^{-}, \mathcal{D}_{K}$ and $\mathcal{R}_{K}$ of $\mathcal{M}(X)$ :

- $\mathcal{W}_{t_{i}, t_{j}}: \mathcal{D}_{\mathcal{W}}^{+} \rightarrow \mathcal{R}_{\mathcal{W}}^{+}$, for $1 \leqslant i<j \leqslant n$;

- $\mathcal{W}_{t_{i}, t_{j}}: \mathcal{D}_{\mathcal{W}}^{-} \rightarrow \mathcal{R}_{\mathcal{W}}^{-}$, for $1 \leqslant j \leqslant i \leqslant n$;

- $K_{t_{i}}: \mathcal{D}_{K} \rightarrow \mathcal{R}_{K}$, for $1 \leqslant i \leqslant n$, with $\mathcal{D}_{K}, \mathcal{R}_{K}$ satisfying

$$
\begin{aligned}
& \mathcal{D}_{K}, \mathcal{R}_{K} \subseteq \mathcal{D}_{\mathcal{W}}^{+} \cap \mathcal{D}_{\mathcal{W}}^{-} \quad \text { and } \quad \mathcal{W}_{t_{i}, t_{j}}\left(\mathcal{D}_{K}\right) \subseteq \mathcal{D}_{\mathcal{W}}^{+} \cap \mathcal{D}_{\mathcal{W}}^{-} \quad \text { for all } i \text { and } j \text {, } \\
& \mathcal{W}_{t_{i}, t_{j}}\left(\mathcal{D}_{K}\right) \subseteq \mathcal{D}_{K} \quad \text { for all } i>j \text {. }
\end{aligned}
$$

Given these operators, we construct an integral operator $K^{\text {ext }}$ acting on the space $\mathcal{D}_{K}^{\left(t_{1}, \ldots, t_{n}\right)}$ of functions $f:\left\{t_{1}, \ldots, t_{n}\right\} \times X \rightarrow \mathbb{R}$ such that $f\left(t_{i}, \cdot\right) \in \mathcal{D}_{K}$ for $1 \leqslant i \leqslant n$ through the following integral kernel:

$$
K^{\operatorname{ext}}\left(t_{i}, x ; t_{j}, y\right)= \begin{cases}\mathcal{W}_{t_{i}, t_{j}} K_{t_{j}}(x, y), & \text { if } i \geqslant j \\ -\mathcal{W}_{t_{i}, t_{j}}\left(I-K_{t_{j}}\right)(x, y), & \text { if } i<j\end{cases}
$$

(that is, for $f \in \mathcal{D}_{K}^{\left(t_{1}, \ldots, t_{n}\right)}$, we set $K^{\text {ext }} f\left(t_{i}, x\right)=\sum_{j=1}^{n} \int_{X} d \mu(y) K^{\text {ext }}\left(t_{i}, x ; t_{j}, y\right) f\left(t_{j}, y\right)$ ). Our assumptions on the domains and ranges of our operators ensure that these (and later) compositions are always well defined.

We will suppose that our operators satisfy the following algebraic assumptions: for all $f \in \mathcal{D}_{K}$,

$$
\begin{aligned}
\mathcal{W}_{t_{i}, t_{i}} f & =f & & \text { for all } 1 \leqslant i \leqslant n, \\
\mathcal{W}_{t_{i}, t_{j}} \mathcal{W}_{t_{j}, t_{k}} f & =\mathcal{W}_{t_{i}, t_{k}} f & & \text { for all } 1 \leqslant i, j, k \leqslant n, \\
\mathcal{W}_{t_{i}, t_{j}} K_{t_{j}} \mathcal{W}_{t_{j}, t_{i}} f & =K_{t_{i}} f & & \text { for all } 1 \leqslant i<j \leqslant n .
\end{aligned}
$$


These three assumptions are our replacement for Assumption 2 of [8]. There are two main differences with that paper: First, we are assuming here that the $\mathcal{W}_{t_{i}, t_{j}}$ 's are properly defined operators for $i>j$, and that $\mathcal{W}_{t_{i}, t_{j}}$ and $\mathcal{W}_{t_{j}, t_{i}}$ are essentially inverses of each other; and second, the reversibility relation $\mathcal{W}_{t_{i}, t_{j}} K_{t_{j}}=K_{t_{i}} \mathcal{W}_{t_{i}, t_{j}}$ which is assumed in [8] is replaced here by (D.3c). $\left.{ }^{34}\right)$

Additionally, we consider multiplication operators $N_{t_{i}}$ acting on $\mathcal{M}(X)$ as $N_{t_{i}} f(x)=$ $\varphi_{t_{i}}(x) f(x)$ for some $\varphi_{t_{i}} \in \mathcal{M}(X)$. We define $N$ to be the diagonal operator acting on functions $f:\left\{t_{1}, \ldots, t_{n}\right\} \times X \rightarrow \mathbb{R}$ as $N f\left(t_{i}, \cdot\right)=N_{t_{i}} f\left(t_{i}, \cdot\right)$. We also introduce the notation

$$
\bar{N}_{t_{i}}=I-N_{t_{i}} .
$$

We need to make some additional assumptions on our operators (the first one is convenient in light of our setting in the next subsection, but could be relaxed as in [8]; the last four replace Assumptions 1 and 3 in [8, Theorem 3.3]):

(i) $\varphi_{t_{i}}(x) \geqslant 0$ for all $x \in X$. We let $N_{t_{i}}^{1 / 2}$ denote the operator of multiplication by $\varphi_{t_{i}}(x)^{1 / 2}$ and define $N^{1 / 2}$ in the same way.

(ii) For all $1 \leqslant i \leqslant n, N_{t_{i}}^{1 / 2}$ maps $L^{2}(X)$ to $\mathcal{D}_{K}$.

(iii) For all $1 \leqslant i, j \leqslant n, N_{t_{i}}^{1 / 2}$ maps $\mathcal{W}_{t_{i}, t_{j}}\left(\mathcal{R}_{K}\right)$ to $L^{2}(X)$, and if additionally $i \geqslant j$ then it also maps $\mathcal{W}_{t_{i}, t_{j}}\left(\mathcal{D}_{K}\right)$ to $L^{2}(X)$;

(iv) $\mathcal{R}_{K} \cup \mathcal{D}_{\mathcal{W}}^{+} \subseteq L^{2}(X)$.

(v) There exist multiplication operators $V_{t_{i}}$ and $V_{t_{i}}^{\prime}$ satisfying $V_{t_{i}}^{\prime} V_{t_{i}} N_{t_{i}}^{1 / 2}=N_{t_{i}}^{1 / 2}$ and such that, for all $i<j$, the operator $V_{t_{i}} N_{t_{i}}^{1 / 2} \mathcal{W}_{t_{i}, t_{j}} N_{t_{j}}^{1 / 2} V_{t_{j}}^{\prime}$ is trace class in $L^{2}(X)$ and, for all $i$ and $j$, the operators $V_{t_{i}} N_{t_{i}}^{1 / 2} \mathcal{W}_{t_{i}, t_{j}} K_{t_{j}} N_{t_{j}}^{1 / 2} V_{t_{j}}^{\prime}$ are trace class in $L^{2}(X)$.

(vi) There exist multiplication operators $U_{t_{i}}$ and $U_{t_{i}}^{\prime}$ satisfying

$$
U_{t_{i}} U_{t_{i}}^{\prime} K_{t_{i}} \mathcal{W}_{t_{i}, t_{j}}=K_{t_{i}} \mathcal{W}_{t_{i}, t_{j}}
$$

for all $j \leqslant i$ and such that the operator

$$
U_{t_{i}}\left[K_{t_{i}} \mathcal{W}_{t_{i}, t_{i+1}} \bar{N}_{t_{i+1}} \ldots \mathcal{W}_{t_{n-1}, t_{n}} \bar{N}_{t_{n}} K_{t_{n}}-K_{t_{i}} \mathcal{W}_{t_{i}, t_{1}} \bar{N}_{t_{1}} \mathcal{W}_{t_{1}, t_{2}} \bar{N}_{t_{2}} \ldots \mathcal{W}_{t_{n-1}, t_{n}} \bar{N}_{t_{n}}\right] U_{t_{i}}^{\prime}
$$

is trace class in $L^{2}(X)$.

TheOrem D.1. In the above setting, assume that (D.1), (D.3a)-(D.3c) and assumptions (i)-(vi) are satisfied. Then,

$$
\begin{aligned}
& \operatorname{det}\left(I-N^{1 / 2} K^{\mathrm{ext}} N^{1 / 2}\right)_{L^{2}\left(\left\{t_{1}, \ldots, t_{n}\right\} \times X\right)} \\
& \quad=\operatorname{det}\left(I-K_{t_{n}}+K_{t_{n}} \mathcal{W}_{t_{n}, t_{1}} \bar{N}_{t_{1}} \mathcal{W}_{t_{1}, t_{2}} \bar{N}_{t_{2}} \ldots \mathcal{W}_{t_{n-1}, t_{n}} \bar{N}_{t_{n}}\right)_{L^{2}(X)} .
\end{aligned}
$$

$\left.{ }^{34}\right)$ It is possible to state a version of Theorem D.1 where $\mathcal{W}_{t_{j}, t_{i}}$ is not necessarily well defined for $i<j$, but instead one assumes that $\mathcal{W}_{t_{j}, t_{i}} K_{t_{i}}$ and $K_{t_{j}} \mathcal{W}_{t_{j}, t_{i}}$ are well defined and satisfy the necessary algebraic assumptions. 
Note that, by (ii) above, the operator inside the determinant on the left-hand side acts on $L^{2}\left(\left\{t_{1}, \ldots, t_{n}\right\} \times X\right)$. Similarly, by (iii) above and the fact that

$$
\begin{aligned}
& K_{t_{n}}-K_{t_{n}} \mathcal{W}_{t_{n}, t_{1}} \bar{N}_{t_{1}} \mathcal{W}_{t_{1}, t_{2}} \bar{N}_{t_{2}} \ldots \mathcal{W}_{t_{n-1}, t_{n}} \bar{N}_{t_{n}} \\
& =\sum_{j=1}^{n} \sum_{k=0}^{n-j}(-1)^{k} \sum_{j=\ell_{0}<\ell_{1}<\ldots<\ell_{k} \leqslant n} K_{n} \mathcal{W}_{n, j} N_{j} \mathcal{W}_{j, \ell_{1}} N_{\ell_{1}} \mathcal{W}_{\ell_{1}, \ell_{2}} N_{\ell_{k-1}} \mathcal{W}_{\ell_{k-1}, \ell_{k}} N_{\ell_{k}} \mathcal{W}_{\ell_{k}, n}
\end{aligned}
$$

(see (D.7) below), the operator inside the determinant on the right-hand side acts on $L^{2}(X)$. Moreover, due to (iv) and (v), after conjugating appropriately by the operators $V_{t_{i}}$ and $V_{t_{i}}^{\prime}$ on the left, and $U_{t_{n}}$ and $U_{t_{n}}^{\prime}$ on the right, which does not change the value of the Fredholm determinants, the operators on both sides become trace class, which shows that the two Fredholm determinants are finite.

Proof of Theorem D.1. The proof is a minor adaptation of the arguments in $[8$, Theorem 3.3], and we will use throughout it all the notation and conventions of that paper. We will just sketch the proof, skipping some of the technical details. In particular, we will completely omit the need to conjugate by the operators $U_{t_{i}}$ and $V_{t_{i}}$, since this aspect of the proof can be adapted straightforwardly from [8] (such conjugations are used to justify the operations involving the multiplicativity and the cyclic property of the Fredholm determinant).

In order to simplify notation throughout the proof, we will replace subscripts of the form $t_{i}$ by $i$, so for example $\mathcal{W}_{i, j}=\mathcal{W}_{t_{i}, t_{j}}$. Let $\mathrm{K}=N^{1 / 2} K^{\text {ext }} N^{1 / 2}$. Then, $\mathrm{K}$ can be written as

$$
\mathrm{K}=\mathrm{N}^{1 / 2}\left(\mathrm{~W}^{-} \mathrm{K}^{\mathrm{d}}+\mathrm{W}^{+}\left(\mathrm{K}^{\mathrm{d}}-\mathrm{I}\right)\right) \mathrm{N}^{1 / 2} \quad \text { with } \mathrm{K}_{i j}^{\mathrm{d}}=K_{i} \mathbf{1}_{i=j} \text { and } \mathrm{N}_{i, j}^{1 / 2}=N_{i}^{1 / 2} \mathbf{1}_{i=j} \text {, }
$$

where $\mathrm{W}^{-}$and $\mathrm{W}^{+}$are lower triangular, respectively strictly upper triangular, and defined by

$$
\mathrm{W}_{i j}^{-}=\mathcal{W}_{i, j} \mathbf{1}_{i \geqslant j} \quad \text { and } \quad \mathrm{W}_{i j}^{+}=\mathcal{W}_{i, j} \mathbf{1}_{i<j}
$$

The key to the proof in [8] was to observe that

$$
\left.\left[\left(\mathbf{I}+\mathrm{W}^{+}\right)^{-1}\right)\right]_{i, j}=I \mathbf{1}_{j=i}-\mathcal{W}_{i, i+1} \mathbf{1}_{j=i+1},
$$

which then implies that

$$
\left[\left(\mathrm{W}^{-}+\mathrm{W}^{+}\right) \mathrm{K}^{\mathrm{d}}\left(\mathrm{I}+\mathrm{W}^{+}\right)^{-1}\right]_{i, j}=\mathcal{W}_{i, 1} K_{1} \mathbf{1}_{j=1} .
$$

The fact that only the first column of this matrix has non-zero entries is what ultimately allows one to turn the Fredholm determinant of an extended kernel into one of a kernel acting on $L^{2}(X)$. However, the derivation of this last identity uses

$$
\mathcal{W}_{i, j-1} K_{j-1} \mathcal{W}_{j-1, j}=\mathcal{W}_{i, j} K_{j}
$$


which is a consequence of Assumptions 2 (ii) and 2 (iii) in [8], and thus is not available to us. In our case, we may proceed similarly by observing that

$$
\left.\left[\left(\mathrm{W}^{-}\right)^{-1}\right)\right]_{i, j}=I \mathbf{1}_{j=i}-\mathcal{W}_{i, i-1} \mathbf{1}_{j=i-1},
$$

as can be checked directly using (D.3a) and (D.3b). Now, using the identity

$$
\mathcal{W}_{i, j+1} K_{j+1} \mathcal{W}_{j+1, j}=\mathcal{W}_{i, j} K_{j}
$$

(which follows from our assumption (D.3c) together with (D.3b)), we get

$$
\left[\left(\mathrm{W}^{-}+\mathrm{W}^{+}\right) \mathrm{K}^{\mathrm{d}}\left(\mathrm{W}^{-}\right)^{-1}\right]_{i, j}=\mathcal{W}_{i, j} K_{j}-\mathcal{W}_{i, j+1} K_{j+1} \mathcal{W}_{j+1, j} \mathbf{1}_{j<n}=\mathcal{W}_{i, n} K_{n} \mathbf{1}_{j=n} .
$$

Note that now only the last column of this matrix has non-zero entries, which accounts for the different expression on the right-hand side of (D.4) when compared to [8]. To take advantage of (D.5), we write

$$
\mathrm{I}-\mathrm{K}=\left(\mathrm{I}+\mathrm{N}^{1 / 2} \mathrm{~W}^{+} \mathrm{N}^{1 / 2}\right)\left[\mathrm{I}-\left(\mathrm{I}+\mathrm{N}^{1 / 2} \mathrm{~W}^{+} \mathrm{N}^{1 / 2}\right)^{-1} \mathrm{~N}^{1 / 2}\left(\mathrm{~W}^{-}+\mathrm{W}^{+}\right) \mathrm{K}^{\mathrm{d}}\left(\mathrm{W}^{-}\right)^{-1} \mathrm{~W}^{-} \mathrm{N}^{1 / 2}\right] .
$$

Since $\mathrm{N}^{1 / 2} \mathrm{~W}^{+} \mathrm{N}^{1 / 2}$ is strictly upper triangular, $\operatorname{det}\left(\mathrm{I}+\mathrm{N}^{1 / 2} \mathrm{~W}^{+} \mathrm{N}^{1 / 2}\right)=1$, which in particular shows that $\mathrm{I}+\mathrm{N}^{1 / 2} \mathrm{~W}^{+} \mathrm{N}^{1 / 2}$ is invertible. Thus, using (D.5), we $\operatorname{deduce}$ that $\operatorname{det}(\mathrm{I}-\mathrm{K}$ ) is the same as

$$
\operatorname{det}\left(\mathrm{I}-\left(\mathrm{I}+\mathrm{N}^{1 / 2} \mathrm{~W}^{+} \mathrm{N}^{1 / 2}\right)^{-1} \mathrm{~N}^{1 / 2} \mathrm{~W}^{(n)} \mathrm{K}^{\mathrm{d}} \mathrm{W}^{-} \mathrm{N}^{1 / 2}\right),
$$

with $\mathrm{W}_{i, j}^{(n)}=\mathcal{W}_{i, n} \mathbf{1}_{j=n}$. Using the cyclic property of the Fredholm determinant we deduce now that $\operatorname{det}(\mathrm{I}-\mathrm{K})=\operatorname{det}(\mathrm{I}-\widetilde{\mathrm{K}})$ with

$$
\widetilde{K}=K^{d} W^{-} N^{1 / 2}\left(I+N^{1 / 2} W^{+} N^{1 / 2}\right)^{-1} N^{1 / 2} W^{(n)} .
$$

Since only the last column of $\mathrm{W}^{(n)}$ is non-zero, the same holds for $\widetilde{K}$, and thus

$$
\operatorname{det}(\mathbf{I}-\mathrm{K})=\operatorname{det}\left(\mathbf{I}-\widetilde{\mathbf{K}}_{n, n}\right)_{L^{2}(X)} \text {. }
$$

Our goal is thus to compute $\widetilde{\mathrm{K}}_{n, n}$. We have, for $0 \leqslant k \leqslant n-i$,

$$
\begin{aligned}
& {\left[\left(\mathrm{N}^{1 / 2} \mathrm{~W}^{+} \mathrm{N}^{1 / 2}\right)^{k} \mathrm{~N}^{1 / 2} \mathrm{~W}^{(n)}\right]_{i, n}} \\
& \quad=\sum_{i<\ell_{1}<\ldots<\ell_{k} \leqslant n} N_{i}^{1 / 2} \mathcal{W}_{i, \ell_{1}} N_{\ell_{1}} \mathcal{W}_{\ell_{1}, \ell_{2}} \ldots N_{\ell_{k-1}} \mathcal{W}_{\ell_{k-1}, \ell_{k}} N_{\ell_{k}} \mathcal{W}_{\ell_{k}, n},
\end{aligned}
$$

while, for $k>n-i$, the left-hand side above equals zero (the case $k=0$ is interpreted as $\left.N_{i}^{1 / 2} \mathcal{W}_{i, n}\right)$. As in [8], this leads to

$$
\widetilde{\mathrm{K}}_{i, n}=\sum_{j=1}^{i} \sum_{k=0}^{n-j}(-1)^{k} \sum_{j=\ell_{0}<\ell_{1}<\ldots<\ell_{k} \leqslant n} K_{i} \mathcal{W}_{i, j} N_{j} \mathcal{W}_{j, \ell_{1}} N_{\ell_{1}} \mathcal{W}_{\ell_{1}, \ell_{2}} N_{\ell_{k-1}} \mathcal{W}_{\ell_{k-1}, \ell_{k}} N_{\ell_{k}} \mathcal{W}_{\ell_{k}, n}
$$

Replacing each $N_{\ell}$ by $I-\bar{N}_{\ell}$ except for the first one and simplifying as in [8] leads to

$$
\widetilde{\mathrm{K}}_{i, n}=K_{i} \mathcal{W}_{i, i+1} \bar{N}_{i+1} \mathcal{W}_{i+1, i+2} \bar{N}_{i+2} \ldots \mathcal{W}_{n-1, n} \bar{N}_{n}-K_{i} \mathcal{W}_{i, 1} \bar{N}_{1} \mathcal{W}_{1,2} \bar{N}_{2} \ldots \mathcal{W}_{n-1, n} \bar{N}_{n}
$$

Setting $i=n$ yields $\widetilde{\mathrm{K}}_{n, n}=K_{n}-K_{n} \mathcal{W}_{n, 1} \bar{N}_{1} \mathcal{W}_{1,2} \bar{N}_{2} \ldots \mathcal{W}_{n-1, n} \bar{N}_{n}$, which, in view of (D.6), yields the result. 


\section{D.2. Proof of the TASEP path integral formula}

Recall that $Q^{n-m} \Psi_{n-k}^{n}=\Psi_{m-k}^{m}$. Then, for $K_{t}^{(n)}=K_{t}(n, \cdot ; n, \cdot)$ we may write

$$
\begin{aligned}
Q^{n_{j}-n_{i}} K_{t}^{\left(n_{j}\right)} & =\sum_{k=0}^{n_{j}-1} Q^{n_{j}-n_{i}} \Psi_{k}^{n_{j}} \otimes \Phi_{k}^{n_{j}} \\
& =\sum_{k=0}^{n_{j}-1} \Psi_{n_{i}-n_{j}+k}^{n_{i}} \otimes \Phi_{k}^{n_{j}} \\
& =K_{t}\left(n_{i}, \cdot ; n_{j}, \cdot\right)+Q^{n_{j}-n_{i}} \mathbf{1}_{n_{i}<n_{j}} .
\end{aligned}
$$

This means that the extended kernel $K_{t}$ defined in (2.6) has exactly the structure specified in (D.2), taking (for fixed $t \geqslant 0$ )

$$
t_{i}=n_{i}, \quad K_{t_{i}}=K_{t}^{\left(n_{i}\right)} \text { and and } \quad \mathcal{W}_{t_{i}, t_{j}}=Q^{n_{j}-n_{i}} .
$$

As a consequence, the path integral version (2.12) of the TASEP formula will follow from Theorem D.1, with $N_{t_{i}}=\bar{\chi}_{a_{i}}$, once we check all the necessary assumptions. We do this next.

First, we need to specify the domains and ranges of our operators:

$$
\begin{array}{cl}
\mathcal{D}_{K}=\left\{f \in \ell^{2}(\mathbb{Z}): \sum_{x \in \mathbb{Z}} 2^{x}\left|x^{k} f(x)\right|<\infty \text { for all } k \geqslant 0\right\}, & \mathcal{R}_{K}=\ell^{2}(\mathbb{Z}), \\
\mathcal{D}_{\mathcal{W}}^{+}=\mathcal{R}_{\mathcal{W}}^{+}=\left\{f \in \ell^{2}(\mathbb{Z}): \sum_{x \leqslant 0} 2^{x}\left|x^{k} f(x)\right|<\infty \text { for all } k \geqslant 0\right\}, & \mathcal{D}_{\mathcal{W}}^{-}=\mathcal{R}_{\mathcal{W}}^{-}=\mathcal{M}(\mathbb{Z}) .
\end{array}
$$

It is easy to check that our operators are indeed well defined with these domains and ranges, and also that (D.1) and assumptions (i)-(iv) are satisfied (essentially all one needs is to observe that $Q$ and $Q^{-1}$ preserve $\ell^{2}(\mathbb{Z})$ ). Before checking assumptions (v) and (vi), let us turn to (D.3).

Conditions (D.3a) and (D.3b) are clearly satisfied. We note at this stage that Assumptions 2 (i) and 2 (ii) in [8, Theorem 3.3] (the semigroup property and the rightinvertibility condition) hold in our setting, but their Assumption 2 (iii), which translates into $Q^{n_{j}-n_{i}} K_{t}^{\left(n_{j}\right)}=K_{t}^{\left(n_{i}\right)} Q^{n_{j}-n_{i}}$ for $n_{i} \leqslant n_{j}$, does not (in fact, the right-hand side does not even make sense as the product is divergent, as can be seen by noting that $\left.\Phi_{0}^{(n)}(x)=2^{x-X_{0}(n)}\right)$, which is why we need Theorem D.1. To use it, we need to check (D.3c), that is

$$
Q^{n_{j}-n_{i}} K_{t}^{\left(n_{j}\right)} Q^{n_{i}-n_{j}}=K_{t}^{\left(n_{i}\right)}
$$

for $n_{i}<n_{j}$. In fact, if $0 \leqslant k<n_{i}$, then (2.14a), together with the easy fact that $h_{k}^{n}(\ell, z)=$ $h_{k-1}^{n-1}(\ell-1, z)$, implies that

$$
\left(Q^{*}\right)^{n_{i}-n_{j}} h_{k+n_{j}-n_{i}}^{n_{j}}(0, z)=h_{k+n_{j}-n_{i}}^{n_{j}}\left(n_{j}-n_{i}, z\right)=h_{k}^{n_{i}}(0, z),
$$


so that $\left(Q^{*}\right)^{n_{i}-n_{j}} \Phi_{k+n_{j}-n_{i}}^{n_{j}}=\Phi_{k}^{n_{i}}$. Therefore, proceeding as in (D.8), the left-hand side of (D.9) equals

$$
\begin{aligned}
\sum_{k=0}^{n_{j}-1} Q^{n_{j}-n_{i}} \Psi_{k}^{n_{j}} \otimes\left(Q^{*}\right)^{n_{i}-n_{j}} \Phi_{k}^{n_{j}} & =\sum_{k=n_{i}-n_{j}}^{n_{i}-1} Q^{n_{j}-n_{i}} \Psi_{k+n_{j}-n_{i}}^{n_{j}} \otimes\left(Q^{*}\right)^{n_{i}-n_{j}} \Phi_{k+n_{j}-n_{i}}^{n_{j}} \\
& =\sum_{k=n_{i}-n_{j}}^{-1} \Psi_{k}^{n_{i}} \otimes\left(Q^{*}\right)^{n_{i}-n_{j}} \Phi_{k+n_{j}-n_{i}}^{n_{j}}+\sum_{k=0}^{n_{i}-1} \Psi_{k}^{n_{i}} \otimes \Phi_{k}^{n_{i}}
\end{aligned}
$$

(note the crucial fact that, while $Q^{*} \Phi_{\ell}^{n}$ is divergent, in this argument $\left(Q^{*}\right)^{m}$ is applied to these functions only for negative $m$ ). The last sum is exactly the right-hand side of (D.9), so we need to check that the first sum on the right-hand side above vanishes. For this, we note that, if $n_{i}-n_{j} \leqslant k<0$, then we have

$$
\left(Q^{*}\right)^{n_{i}-n_{j}} h_{k+n_{j}-n_{i}}^{n_{j}}(0, z)=\left(Q^{*}\right)^{k} h_{k+n_{j}-n_{i}}^{n_{j}}\left(k+n_{j}-n_{i}, z\right)=0,
$$

due to $(2.14 \mathrm{~b})$ and the fact that $2^{z} \in \operatorname{ker}\left(Q^{*}\right)^{-1}$, which gives $\left(Q^{*}\right)^{n_{i}-n_{j}} \Phi_{k+n_{j}-n_{i}}^{n_{j}}=0$, as desired.

To finish our proof of (2.12), we need to check that conditions (v) and (vi) in Theorem D.1 are satisfied. For (v), we let $V_{n_{i}}=\widetilde{V}_{i}$ and $V_{n_{i}}^{\prime}=\widetilde{V}_{i}^{\prime}$, with $\widetilde{V}_{i} f(x)=\left(1+x^{2}\right)^{i}$ and $\widetilde{V}_{i}^{\prime}(x)=\left(1+x^{2}\right)^{-i}$. For $i<j$, we need to check that $\bar{\chi}_{a_{i}} V_{n_{i}} Q^{n_{j}-n_{i}} V_{n_{j}}^{\prime} \bar{\chi}_{a_{j}}$ is trace class. The $\bar{\chi}_{a_{i}}$ 's are projections, so $\left\|\bar{\chi}_{a_{i}}\right\|_{\infty}=1$ and thus it is enough to show that $V_{n_{i}} Q^{n_{j}-n_{i}} V_{n_{j}}^{\prime}$ is trace class. Assume first that $n_{j}-n_{i}>1$. Then, we may write $V_{n_{i}} Q^{n_{j}-n_{i}} V_{n_{j}}^{\prime}$ as

$$
\prod_{\ell=n_{i}}^{n_{j}-1} \widetilde{V}_{\ell} Q \widetilde{V}_{\ell+1}^{\prime}
$$

and it is enough to show that each factor is Hilbert-Schmidt, which is clear:

$$
\left\|\widetilde{V}_{\ell} Q \widetilde{V}_{\ell+1}^{\prime}\right\|_{2}^{2}=\sum_{\substack{x, y \in \mathbb{Z} \\ y<x}} 2^{2(y-x)} \frac{\left(1+x^{2}\right)^{2 \ell}}{\left(1+y^{2}\right)^{2(\ell+1)}}=\sum_{y \in \mathbb{Z}} \frac{1}{\left(1+y^{2}\right)^{2(\ell+1)}} \sum_{x>0}\left(1+(x+y)^{2}\right)^{2 \ell} 2^{-2 x}<\infty .
$$

If $n_{j}-n_{i}=1$, then we write $Q(x, y-1)=A^{2}(x, y)$ with

$$
A(x, y)=\frac{\Gamma\left(x-y+\frac{1}{2}\right)}{\sqrt{\pi}(x-y) !} 2^{-(x-y+1 / 2)} \mathbf{1}_{x \geqslant y}
$$

(this can be checked directly, but it just corresponds to writing a Geom $\left[\frac{1}{2}\right]$ random variable as the sum of two independent negative binomial random variables with parameters $\left.\left(\frac{1}{2}, \frac{1}{2}\right)\right)$, and thus it is enough to show that $V_{i} A^{2} V_{i+1}^{\prime}$ is trace class. By Stirling's formula,

$$
A(x, y) \sim c(x-y)^{-1 / 2} 2^{-(x-y)} \quad \text { as } x-y \rightarrow \infty,
$$


so factoring the kernel as $\left(V_{i} A V_{i+1 / 2}^{\prime}\right)\left(V_{i+1 / 2} A V_{i+1}^{\prime}\right)$, the above argument still applies. Next, for all $i$ and $j$, we need to check that $\bar{\chi}_{a_{i}} V_{n_{i}} Q^{n_{j}-n_{i}} K_{t}^{\left(n_{i}\right)} \bar{\chi}_{a_{j}} V_{n_{j}}^{\prime}$ is trace class. Since

$$
Q^{n_{j}-n_{i}} K_{t}^{\left(n_{j}\right)}=\sum_{k=1}^{n_{j}} Q^{n_{j}-n_{i}} \Psi_{n_{j}-k}^{n_{j}} \otimes \Phi_{n_{j}-k}^{n_{j}},
$$

it is enough to show (using also (2.9)) that $\bar{\chi}_{a_{i}} V_{n_{i}} \Psi_{n_{i}-k}^{n_{i}} \otimes \bar{\chi}_{a_{j}} V_{n_{j}}^{\prime} \Phi_{n_{j}-k}^{n_{j}}$ is trace class for each $k=1, \ldots, n_{j}$. This operator is rank-1 and its unique eigenvalue is

$$
\lambda=\sum_{x \in \mathbb{Z}} \bar{\chi}_{a_{i}} V_{n_{i}} \Psi_{n_{i}-k}^{n_{i}}(x) \bar{\chi}_{a_{j}} V_{n_{j}}^{\prime} \Phi_{n_{j}-k}^{n_{j}}(x),
$$

which satisfies

$$
|\lambda|^{2} \leqslant \sum_{x \leqslant a_{i}}\left(1+x^{2}\right)^{2 i} \Psi_{n_{i}-k}^{n_{i}}(x)^{2} \sum_{x \leqslant a_{j}} \frac{2^{2 x}}{\left(1+x^{2}\right)^{2 j}}\left(2^{-x} \Psi_{n_{i}-k}^{n_{i}}(x)\right)^{2}<\infty,
$$

because $\Psi_{n_{i}-k}^{n_{i}}(x)=0$ for $x<X_{0}(k)-n_{i}+k$ and $2^{-x} \Psi_{n_{i}-k}^{n_{i}}(x)$ is a polynomial. Therefore,

$$
\left\|\bar{\chi}_{a_{i}} V_{n_{i}} \Psi_{n_{i}-k}^{n_{i}} \otimes \bar{\chi}_{a_{j}} V_{n_{j}}^{\prime} \Phi_{n_{j}-k}^{n_{j}}\right\|_{1}=|\lambda|<\infty
$$

as desired.

We turn finally to (vi). By (D.7), it is enough to show that

$$
U_{n_{i}} K_{t}^{\left(n_{i}\right)} Q^{n_{\ell_{0}}-n_{i}} \bar{\chi}_{a_{\ell_{0}}} Q^{n_{\ell_{1}}-n_{\ell_{0}}} \bar{\chi}_{a_{\ell_{1}}} \ldots Q^{n_{\ell_{k}}-n_{\ell_{k-1}}} \bar{\chi}_{a_{\ell_{k}}} Q^{m-n_{\ell_{k}}} U_{n_{m}}^{\prime}
$$

is trace class for all $\ell_{0}<\ell_{1}<\ldots<\ell_{k} \leqslant m$ with $\ell_{0}=1, \ldots, i$ and $k=0, \ldots, m-\ell_{0}$, where we choose $U_{n_{i}}=V_{n_{i}}$ and $U_{n_{i}}^{\prime}=V_{n_{i}}^{\prime}$. Now, replace each $\bar{\chi}_{a_{\ell}}$ in the above operator by $V_{n_{\ell}}^{\prime} \bar{\chi}_{a_{\ell}} V_{n_{\ell}}$. The argument in the last paragraph can be used to show again that

$$
U_{n_{i}} K_{t}^{\left(n_{i}\right)} Q^{n_{\ell_{0}}-n_{i}} V_{n_{\ell_{0}}}^{\prime} \bar{\chi}_{a_{\ell_{0}}}
$$

is trace class (now using the decay of $\Psi_{n_{i}-k}^{n_{i}}(x)$ as $x \rightarrow \infty$, since we have no projection on the left). As shown above, the rest is the product of trace class operators, so the whole product (D.10) is trace class as needed.

Acknowledgements. J.Q. and K. M. were supported by the Natural Sciences and Engineering Research Council of Canada. J. Q. was also supported by a Killam research fellowship. D. R. was supported by Centro de Modelamiento Matemático (CMM) Basal Funds AFB170001, ACE210010 and FB210005 from ANID-Chile, by Programa Iniciativa Científica Milenio grant number NC120062 through Nucleus Millenium Stochastic Models of Complex and Disordered Systems, and by Fondecyt Grants 1160174 and 1201914. 


\section{References}

[1] Alberts, T., Khanin, K. \& Quastel, J., The intermediate disorder regime for directed polymers in dimension 1+1. Ann. Probab., 42 (2014), 1212-1256.

[2] Amir, G., Corwin, I. \& Quastel, J., Probability distribution of the free energy of the continuum directed random polymer in 1+1 dimensions. Comm. Pure Appl. Math., 64 (2011), 466-537.

[3] Baik, J., Ferrari, P.L. \& PÉché, S., Limit process of stationary TASEP near the characteristic line. Comm. Pure Appl. Math., 63 (2010), 1017-1070.

[4] BAIK, J. \& LiU, Z., Multipoint distribution of periodic TASEP. J. Amer. Math. Soc., 32 (2019), 609-674.

[5] Baik, J. \& Rains, E. M., Symmetrized random permutations, in Random Matrix Models and their Applications, Math. Sci. Res. Inst. Publ., 40, pp. 1-19. Cambridge Univ. Press, Cambridge, 2001.

[6] Bertini, L. \& Giacomin, G., Stochastic Burgers and KPZ equations from particle systems. Comm. Math. Phys., 183 (1997), 571-607.

[7] Billingsley, P., Convergence of Probability Measures. Wiley Series in Probability and Statistics: Probability and Statistics. John Wiley \& Sons, New York, 1999.

[8] Borodin, A., Corwin, I. \& Remenik, D., Multiplicative functionals on ensembles of non-intersecting paths. Ann. Inst. Henri Poincaré Probab. Stat., 51 (2015), 28-58.

[9] Borodin, A., Ferrari, P. L. \& Prähofer, M., Fluctuations in the discrete TASEP with periodic initial configurations and the Airy ${ }_{1}$ process. Int. Math. Res. Pap. IMRP, 1 (2007), Art. ID rpm002, 47 pp.

[10] Borodin, A., Ferrari, P. L., Prähofer, M. \& Sasamoto, T., Fluctuation properties of the TASEP with periodic initial configuration. J. Stat. Phys., 129 (2007), 1055-1080.

[11] Borodin, A., Ferrari, P. L. \& Sasamoto, T., Transition between Airy and Airy $_{2}$ processes and TASEP fluctuations. Comm. Pure Appl. Math., 61 (2008), 1603-1629.

[12] — Two speed TASEP. J. Stat. Phys., 137 (2009), 936-977.

[13] Borodin, A., Gorin, V. \& Wheeler, M., Shift-invariance for vertex models and polymers. Preprint, 2019. arXiv: 1912.02957 [math.PR].

[14] Burgers, J., The Nonlinear Diffusion Equation: Asymptotic Solutions and Statistical Problems. Springer, Netherlands, 1974.

[15] Calvert, J., Hammond, A. \& Hedge, M., Brownian structure in the KPZ fixed point. Preprint, 2019. arXiv: 1912.00992 [math.PR].

[16] Corwin, I., Ferrari, P. L. \& PÉchÉ, S., Limit processes for TASEP with shocks and rarefaction fans. J. Stat. Phys., 140 (2010), 232-267.

[17] Corwin, I., Liu, Z. \& WAng, D., Fluctuations of TASEP and LPP with general initial data. Ann. Appl. Probab., 26 (2016), 2030-2082.

[18] Corwin, I. \& NicA, M., Intermediate disorder directed polymers and the multi-layer extension of the stochastic heat equation. Electron. J. Probab., 22 (2017), Paper No. 13, 49 pp.

[19] Corwin, I., Quastel, J. \& Remenik, D., Continuum statistics of the Airy 2 process. Comm. Math. Phys., 317 (2013), 347-362.

[20] - Renormalization fixed point of the KPZ universality class. J. Stat. Phys., 160 (2015), 815-834.

[21] Corwin, I., Shen, H. \& Tsai, L. C., $\operatorname{ASEP}(q, j)$ converges to the KPZ equation. Ann. Inst. Henri Poincaré Probab. Stat., 54 (2018), 995-1012.

[22] Corwin, I. \& Tsai, L. C., KPZ equation limit of higher-spin exclusion processes. Ann. Probab., 45 (2017), 1771-1798. 
[23] Dauvergne, D., Ortmann, J. \& Virág, B., The directed landscape. To appear in Acta Mathematica.

[24] Ferrari, P. L., Dimers and orthogonal polynomials: connections with random matrices, in Dimer Models and Random Tilings, Panor. Synthèses, 45, pp. 47-79. Soc. Math. France, Paris, 2015.

[25] Ferrari, P. L. \& Spohn, H., Scaling limit for the space-time covariance of the stationary totally asymmetric simple exclusion process. Comm. Math. Phys., 265 (2006), 1-44. Correction in $45-46$.

[26] Forster, D., Nelson, D. R. \& Stephen, M. J., Large-distance and long-time properties of a randomly stirred fluid. Phys. Rev. A, 16 (1977), 732-749.

[27] Frachebourg, L. \& Martin, P. A., Exact statistical properties of the Burgers equation. J. Fluid Mech., 417 (2000), 323-349.

[28] Gessel, I. \& Viennot, G., Binomial determinants, paths, and hook length formulae. Adv. in Math., 58 (1985), 300-321.

[29] Hairer, M., A theory of regularity structures. Invent. Math., 198 (2014), 269-504.

[30] Hairer, M. \& Quastel, J., A class of growth models rescaling to KPZ. Forum Math. Pi, 6 (2018), Paper No. e3, 112 pp.

[31] Hammond, A., A patchwork quilt sewn from Brownian fabric: regularity of polymer weight profiles in Brownian last passage percolation. Forum Math. Pi, 7 (2019), Paper No. e2, $69 \mathrm{pp}$.

[32] Imamura, T. \& Sasamoto, T., Fluctuations of the one-dimensional polynuclear growth model with external sources. Nuclear Phys. B, 699 (2004), 503-544.

[33] Johansson, K., Shape fluctuations and random matrices. Comm. Math. Phys., 209 (2000), 437-476.

[34] - Discrete polynuclear growth and determinantal processes. Comm. Math. Phys., 242 (2003), 277-329.

[35] - Two time distribution in Brownian directed percolation. Comm. Math. Phys., 351 (2017), 441-492.

[36] - The two-time distribution in geometric last-passage percolation. Probab. Theory Related Fields, 175 (2019), 849-895.

[37] Johansson, K. \& Rahman, M. Multitime distribution in discrete polynuclear growth, 2021.

[38] Kardar, M., Parisi, G. \& Zhang, Y.-C., Dynamical scaling of growing interfaces. Phys. Rev. Lett., 56 (1986), 889-892.

[39] Karlin, S. \& McGregor, G., Scale invariance of the PNG droplet and the Airy process. J. Stat. Phys., 108 (2002), 1071-1106.

[40] Liggett, T.M., Interacting Particle Systems. Grundlehren der Mathematischen Wissenschaften, 276. Springer, New York, 1985.

[41] Matetski, K. \& Remenik, D., TASEP and generalizations: Method for exact solution. Preprint, 2021. arXiv: 2107.07984 [math.PR] .

[42] Moreno Flores, G., Quastel, J. \& Remenik, D., Intermediate disorder limits for directed polymers with boundary conditions. In preparation.

[43] Nica, M., Quastel, J. \& Remenik, D., One-sided reflected Brownian motions and the KPZ fixed point. Forum Math. Sigma, 8 (2020), Paper No. e63, 16 pp.

[44] Pimentel, L. P. R., Ergodicity of the KPZ fixed point. ALEA Lat. Am. J. Probab. Math. Stat., 18 (2021), 963-983.

[45] Prähofer, M. \& Spohn, H., Scale invariance of the PNG droplet and the Airy process. J. Stat. Phys., 108 (2002), 1071-1106. 
[46] Prolhac, S., Spectrum of the totally asymmetric simple exclusion process on a periodic lattice-first excited states. J. Phys. A, 47 (2014), 375001, 29 pp.

[47] Prolhac, S. \& Spohn, H., The one-dimensional KPZ equation and the Airy process. J. Stat. Mech. Theory Exp., (2011), P03020, 15 pp.

[48] Quastel, J. \& Remenik, D., Local behavior and hitting probabilities of the Airy 1 process. Probab. Theory Related Fields, 157 (2013), 605-634.

[49] - Supremum of the Airy2 process minus a parabola on a half line. J. Stat. Phys., 150 (2013), 442-456.

[50] - Airy processes and variational problems, in Topics in Percolative and Disordered Systems, Springer Proc. Math. Stat., 69, pp. 121-171. Springer, New York, 2014.

[51] - How flat is flat in random interface growth? Trans. Amer. Math. Soc., 371 (2019), 6047-6085.

[52] Quastel, J. \& Valkó, B., KdV preserves white noise. Comm. Math. Phys., 277 (2008), 707-714.

[53] Sarkar, S. \& Virág, B., Brownian absolute continuity of the KPZ fixed point with arbitrary initial condition. Ann. Probab., 49 (2021), 1718-1737.

[54] Sasamoto, T., Spatial correlations of the 1D KPZ surface on a flat substrate. J. Phys. A, 38 (2005), L549-L556.

[55] Sasamoto, T. \& Imamura, T., Fluctuations of the one-dimensional polynuclear growth model in half-space. J. Stat. Phys., 115 (2004), 749-803.

[56] SснӥтZ, G. M., Exact solution of the master equation for the asymmetric exclusion process. J. Stat. Phys., 88 (1997), 427-445.

[57] SeppäläInen, T., Hydrodynamic scaling, convex duality and asymptotic shapes of growth models. Markov Process. Related Fields, 4 (1998), 1-26.

[58] Simon, B., The $P(\phi)_{2}$ Euclidean (Quantum) Field Theory. Princeton Series in Physics. Princeton Univ. Press, Princeton, NJ, 1974.

[59] — Trace Ideals and their Applications. Mathematical Surveys and Monographs, 120. Amer. Math. Soc., Providence, RI, 2005.

[60] Tracy, C. A. \& Widom, H., Level-spacing distributions and the Airy kernel. Comm. Math. Phys., 159 (1994), 151-174.

[61] — On orthogonal and symplectic matrix ensembles. Comm. Math. Phys., 177 (1996), 727754 .

[62] - A Fredholm determinant representation in ASEP. J. Stat. Phys., 132 (2008), 291-300.

[63] - Integral formulas for the asymmetric simple exclusion process. Comm. Math. Phys., 279 (2008), 815-844. Correction in Comm. Math. Phys., 304 (2011), 875-878.

[64] - Asymptotics in ASEP with step initial condition. Comm. Math. Phys., 290 (2009), 129-154. 
Konstantin Matetski

Department of Mathematics

University of Toronto

40 St. George Street

Toronto, ON

Canada M5S 2E4

matetski@math.toronto.edu
JEREMY QUASTEL

Department of Mathematics

University of Toronto

40 St. George Street

Toronto, ON

Canada M5S 2E4

quastel@math.toronto. edu

DANIEL REMENIK

Departamento de Ingeniería Matemática and Centro de Modelamiento Matemático (IRL-CNRS 2807)

Universidad de Chile

Av. Beauchef 851, Torre Norte, Piso 5

Santiago

Chile

dremenik@dim.uchile.cl

Received December 5, 2018

Received in revised form April 2, 2020 